\title{
Development of mechanism-based models for resistance spot weld failure simulation of multi-material advanced high strength steel sheets
}

\section{by \\ Daniel Dorribo Dorribo}

ADVERTIMENT La consulta d'aquesta tesi queda condicionada a l'acceptació de les següents condicions d'ús: La difusió d'aquesta tesi per mitjà del repositori institucional UPCommons (http://upcommons.upc.edu/tesis) i el repositori cooperatiu TDX (http://www.tdx.cat/) ha estat autoritzada pels titulars dels drets de propietat intel-lectual únicament per a usos privats emmarcats en activitats d'investigació i docència. No s'autoritza la seva reproducció amb finalitats de lucre ni la seva difusió i posada a disposició des d'un lloc aliè al servei UPCommons o TDX. No s'autoritza la presentació del seu contingut en una finestra o marc aliè a UPCommons (framing). Aquesta reserva de drets afecta tant al resum de presentació de la tesi com als seus continguts. En la utilització o cita de parts de la tesi és obligat indicar el nom de la persona autora.

ADVERTENCIA La consulta de esta tesis queda condicionada a la aceptación de las siguientes condiciones de uso: La difusión de esta tesis por medio del repositorio institucional UPCommons (http://upcommons.upc.edu/tesis) y el repositorio cooperativo TDR (http://www.tdx.cat/?localeattribute=es) ha sido autorizada por los titulares de los derechos de propiedad intelectual únicamente para usos privados enmarcados en actividades de investigación y docencia. No se autoriza su reproducción con finalidades de lucro ni su difusión y puesta a disposición desde un sitio ajeno al servicio UPCommons No se autoriza la presentación de su contenido en una ventana o marco ajeno a UPCommons (framing). Esta reserva de derechos afecta tanto al resumen de presentación de la tesis como a sus contenidos. En la utilización o cita de partes de la tesis es obligado indicar el nombre de la persona autora.

WARNING On having consulted this thesis you're accepting the following use conditions: Spreading this thesis by the institutional repository UPCommons (http://upcommons.upc.edu/tesis) and the cooperative repository TDX (http://www.tdx.cat/?localeattribute=en) has been authorized by the titular of the intellectual property rights only for private uses placed in investigation and teaching activities. Reproduction with lucrative aims is not authorized neither its spreading nor availability from a site foreign to the UPCommons service. Introducing its content in a window or frame foreign to the UPCommons service is not authorized (framing). These rights affect to the presentation summary of the thesis as well as to its contents. In the using or citation of parts of the thesis it's obliged to indicate the name of the author. 
Universitat Politècnica de Catalunya Programa de Doctorat d'Enginyeria Civil

E. T. S. D'Enginyers de Camins, Canals i Ports de Barcelona in collaboration with

SEAT S.A.

DEVELOPMENT OF MECHANISM-BASED MODELS FOR RESISTANCE SPOT WELD FAILURE SIMULATION OF MULTI-MATERIAL ADVANCED HIGH STRENGTH STEEL SHEETS

by

DANIEL DORRIBO DORRIBO

Doctoral Thesis

Advisor: Irene Arias

Barcelona, September 28, 2017 

Aos meus pais e á miña avoa Fina. 



\section{Abstract \\ Development of mechanism-based models for resistance spot weld failure simulation of multi-material advanced high strength steel sheets}

\section{Daniel Dorribo Dorribo}

The automotive industry is constantly involved in the development of new projects aimed at reducing weight, fuel consumptions and costs while improving passengers safety. In order to achieve these increasing demands, Advanced High Strength Steels (AHSS) have been introduced in recent years reducing vehicle structure weights and improving the crashworthiness. With the increase in the bearing capacity of crashrelevant structural components, the sheet metal joining techniques such as adhesive bonding and resistance spot welding (RSW) become critical. In order to develop the vehicle structure in these new projects, full-vehicle crash finite element simulations are usually performed. Simplified beam-like models are currently used in these simulations (with thousands of spot welds) to represent RSW joints response. The maximum bearing force of these models are fitted using large experimental campaigns, considering all the main factors that have the highest influence on the fracture response of a welded joint. The objective of this thesis is twofold: (1) to develop a model that is able to partially replace the extensive experimental campaign in providing parameters for the crash simulation simplified spot weld models, and (2) to gain understanding of spot weld joints failure response in order to improve the current simplified models.

To achieve these objectives, a detailed spot weld model for the prediction of spot weld failure in joints in AHSS sheets is presented. The presented model includes a definition of the local material properties as well as the geometry features of a spot weld. In addition, an industrially suited fracture criterion, i.e. robust and without a long-term calibration, is used for the prediction of maximum force. An energetic fracture criterion based on the use of elastic-plastic fracture-mechanics is identified as the better suited for the prediction of spot weld failure and joint bearing capacity. The J-integral is evaluated in the weld notch and this value is compared with a material parameter, the fracture toughness, in order to obtain the joint maximum force.

The presented detailed FE spot weld model is validated to joints of two different 
steel grades of the AHSS family usually present in the current vehicle structure, a hot formed martensitic boron-alloyed steel (22MnB5) and a cold formed dual phase steel (DP 980). The validation is performed comparing the maximum forces obtained with the finite element model and the results extracted from an extensive loading test experimental campaign where the main factors that have an influence in the spot weld fracture response are considered. The obtained simulated critical forces of the loading tests present good agreement with the experimental ones in all tested configurations.

Finally, based on the presented finite element spot weld model, some recommendations are exposed for extending the model for new combinations and loading conditions. The proposed procedure can be used to reduce the long-term characterization campaigns used to calibrate the joints of a new AHSS grade, where fracture is triggered by stress concentration ahead of a notch. Furthermore, some recommendations for the future structure design are given taking into account the information obtained with the present model. 


\section{Acknowledgements}

The work presented in this thesis has been carried out mainly at the department of Technical Computing of SEAT S.A. and at the Laboratory of Computational Methods and Numerical Analysis (LaCàN) of the University of Catalunya (UPCBarcelonaTech), both located in Barcelona, (in the moment I am writing this text) Spain. It must be said that this collaboration could be done thanks to the the Doctorats Industrials programm of the Generalitat de Catalunya. Furthermore, I have done several stays at the headquarters of the Volkswagen Group in Wolfsburg, Germany, where I spent around half a year. For these reasons I've met many different people and I've been helped by all of them, and I would like to thank all of them.

Everything started with a Skype conversation from Coruña, with my first boss, Xavi Agustin and my academy supervisor, Irene Arias. I would like to thank both of them for the trust they placed in me. I decided to move to Barcelona, to start the new journey that finishes now. It was not easy to start a new scientific research from a industry department not used to this type of work, but with the support from my industrial supervisor Xabi Larráyoz from Wolfsburg I could manage to advance (en el departamento todo el mundo recordará nuestras conversaciones telefónicas de más de una hora en la que se nos calentaba la oreja). I really want to thank him for the help, the support and the supervision of all these years.

I would like to thank my academical supervisors, mainly Irene Arias for the supervision of my work in all these years and Pedro Diez for the not interested support during the maternity leave time of Irene. I am completely sure that this thesis is much better due to the valuable ideas given by both of them, even taking into account that this work is a little far away from the usual research field of both of them.

I would also like to express my gratitude to the people that helped me during my stays in the department of CAE-Methods of Volkswagen Group Research, specially Lars Greve and Michael Andres. Danke für die regularän Termine, euren Räter waren wirklich sehr Hilfreich für meine Arbeit. Danke auch für die speziallen Implementationen in der Code. I must also say thank you to Tom Eller, you gave me crucial data and information about my research topic. I must also thank to all the people that helped me with the experimental parts of my work, again Tom for supporting me with the experimental campaign, but also Josse Scheele and Thomas Noack to help me to perform the tests and Thomas Ludewig for supporting them.

I cannot forget all my colleges of SEAT, specially my boss Xavi Castillo that gave me always good advices and kept an eye on all the difficulties I had. I would like also to thank all the other $\mathrm{PhD}$ candidates of SEAT, all of them really understand the meaning of being the first promotion in such an interesting experience, Adrian, Lluc, Armand, Javi, Carlos, Jorge, Miguel recordaré sempre els nostres dinars de doctorands anònims comentant las nostras penes $i$ allegries.

I cannot forget all the LaCàN colleges; Omid, Aleks, Raquel, Ceren, Raúl from the beginning and Drhuba, Magnus, Daobing, Albert, Olga, David and the Italian community during the last months. It was very pleasureful to work with all of them, 
even taking into account that I was that guy that just came to work a couple of days in the week. I really enjoyed some very interesting conversations during lunch in the $\mathrm{C} 2$ terrace, and I really miss them now.

And not so related with the work, but also very important for it, I also would like to thank the friends that were on my side in Barcelona, sometimes one beer or one coffee can make you progress more than a couple of hours of work, thank you Roi, Alex, Joan, César, Mariña, Lydia and Nacho por todas esas historias que escuchasteis sobre mi tesis sin (casi) quejarse, ;-).

And last, but not least I specially very warm thank you to all my family, moitas grazas por aturarme, moitas grazas a Fina, aos meus pais porque sen eles non podía ter chegado ata aquí, perdoade por as malas constestacións e o mal humor por causa da tese, grazas a miña irmá que de seguro será unha doutora das que salvan vidas. Grazas por suposto ao meu to por meterme no mundo da enxeera e meus padrios e avs que sempre me falaron da lonxana Alemania. Moitas grazas especialmente a Elena, esa persoa que estivo ao meu carón todos estes anos cunhas palabras sempre de ánimo aínda que as circunstancias non foran sempre as desexadas. 


\section{Contents}

\begin{tabular}{|ll}
\hline Abstract & V \\
\hline
\end{tabular}

Contents $\quad$ ix

$\begin{array}{lr}\text { List of Figures } & \text { xi }\end{array}$

$\begin{array}{ll}\text { Nomenclature } & \text { XV }\end{array}$

Latin symbols . . . . . . . . . . . . . . . . . . . . . . . . . $\mathrm{xV}$

Greek symbols . . . . . . . . . . . . . . . . . . . . . . . . . . xvi

Acronyms . . . . . . . . . . . . . . . . . . . . . . . . . . . . xvii

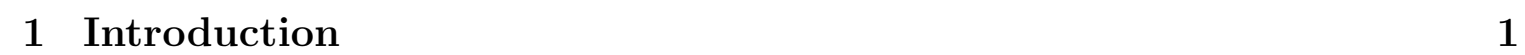

1.1 Motivation . . . . . . . . . . . . . . . . . . . . . . . 1

1.2 Objectives . . . . . . . . . . . . . . . . . . . . . . . . . . 2

1.3 Work approach . . . . . . . . . . . . . . . . . . . . . . . . . . 3

1.4 Chapter overview . . . . . . . . . . . . . . . . . . . . . . . . . . . . . 5

$\begin{array}{llr}2 & \text { State of the } \text { art } & 9\end{array}$

2.1 Vehicle crash tests . . . . . . . . . . . . . . . . . . . . . . 10

2.2 The Advanced High Strength Steels family . . . . . . . . . . . . . . . 11

2.3 Resistance spot welding . . . . . . . . . . . . . . . . . . . 13

2.4 Experimental characterization of spot weld failure . . . . . . . . . . . 17

2.5 Spot weld fracture modeling . . . . . . . . . . . . . . . . . . . . . . 20

$\begin{array}{llr}3 & \text { Resistance Spot Weld model } & 25\end{array}$

3.1 Governing equations . . . . . . . . . . . . . . . . . . . . . . . . 26

3.2 Constitutive model . . . . . . . . . . . . . . . . . . . . . . . . . . . . 26

3.3 Local material properties . . . . . . . . . . . . . . . . . . . . . 27

3.4 Geometry and mesh description . . . . . . . . . . . . . . . . . . . . . 28

3.5 Boundary conditions . . . . . . . . . . . . . . . . . . . . . . . 30

3.6 Spot weld fracture modeling in AHSS . . . . . . . . . . . . . . . . . . 31

3.7 Numerical issues . . . . . . . . . . . . . . . . . . . . . . . . . . . . . 40 
3.8 Preliminary results and loading cases . . . . . . . . . . . . . . . . . 41

$4 \quad$ Application in resistance spot welds of a hot formed steel 45

$4.1 \quad$ Experimental validation campaign . . . . . . . . . . . . 46

$\begin{array}{lll}4.2 & \text { Geometry and local material properties distribution of the spot welds } 50\end{array}$

4.3 Characterization of nugget material properties . . . . . . . . . 55

4.4 Experimental loading tests of the joining specimens . . . . . . . . . . 58

4.5 Model validation $\ldots \ldots \ldots \ldots \ldots$. . . . . . . . . . . . . . 67

5 Application in resistance spot welds of a cold formed steel $\quad 75$

$5.1 \quad$ Experimental validation campaign . . . . . . . . . . . . 76

$\begin{array}{lll}5.2 & \text { Geometry and local material properties distribution of the spot welds } \quad 77\end{array}$

$5.3 \quad$ Characterization of nugget material properties . . . . . . . . . . . . 79

5.4 Experimental loading tests of the joining specimens . . . . . . . . . 83

5.5 Model validation . . . . . . . . . . . . . . . . . 87

\begin{tabular}{|lll}
6 & Application in resistance spot welds of two different steel grades & 91
\end{tabular}

$6.1 \quad$ Experimental validation campaign . . . . . . . . . . . . 92

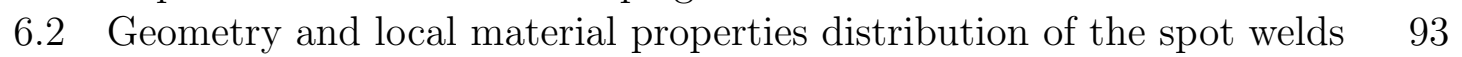

6.3 Characterization of nugget material properties . . . . . . . . . . 95

6.4 Experimental loading tests of the joining specimens . . . . . . . . . . 98

6.5 Model validation . . . . . . . . . . . . . . . . . . . . 103

\begin{tabular}{|lll}
\hline 7 & Model application in real vehicle crash simulations & 111
\end{tabular}

7.1 Steel sheet combinations in vehicle structure . . . . . . . . . . . . . . 112

7.2 Loading conditions during crash situations . . . . . . . . . . . . . . . 114

$7.3 \quad$ Differences between idealized and actual spot welds . . . . . . . . . . . 114

\begin{tabular}{lll}
\hline 8 & Conclusions & 117
\end{tabular}

A Implementation of the $J$-integral calculation in the FE framework 121

A.1 Evaluation of $J_{1}$ using the equivalent domain integral technique . . . 122

A.2 Evaluation of the second integral of Eq. A.2, $J_{W} \ldots \ldots \ldots$. . . . . . 126

A.3 $\quad$ Evaluation of the third integral of Eq. $\mid$ A.2, $J_{A} \ldots \ldots \ldots$

B Reduction of quasi-static solutions CPU Time in a explicit dynamic code

Bibliography

133 


\section{List of Figures}

1.1 Proposed work approach. . . . . . . . . . . . . . . . . . . 5

2.1 Full vehicle model of an Euro NCAP frontal crash. . . . . . . . . . . . . 10

2.2 Body-in-White (BiW) of one modern vehicle. . . . . . . . . . . . . . 11

2.3 Global formability diagram for todays's advanced high strength steelss(AHSSs)

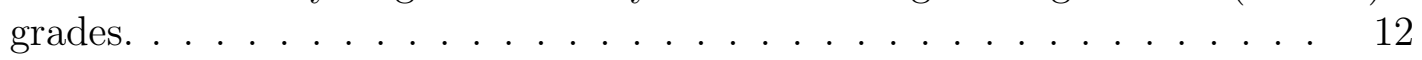

$2.4 \quad$ Schematic representation of resistance spot welding process. . . . . . . . 14

2.5 Typical hardness profile of resistance spot welds of different steel grades. 15

2.6 Schematic resistance spot weld cross sections with the main geometrical

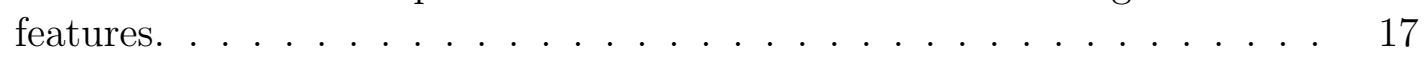

2.7 Cross-sectioned spot weld with a welding void or pore. . . . . . . . . . . 17

2.8 Sketch of the Cross Tension (left) and Tensile Shear (right) tests. . . . . 18

2.9 Scheme of the multiple failure modes represented in cross sectioned spot welds. . . . . . . . . . . . . . . . . . . . . . . 19

2.10 Schematic spot weld cross section with different potential failure zones. $\quad 20$

2.11 Fracture modes. . . . . . . . . . . . . . . . . . . . 24

3.1 Scheme of cross sectioned spot weld with the modeling zones. . . . . . . 28

3.2 Mesh of the loading specimen and the spot weld model. . . . . . . . . . . 29

$3.3 \quad$ Strain field around weld notch for two different shapes. . . . . . . . . . . . 30

3.4 Scheme of loading angles and boundary conditions. . . . . . . . . . . . . 31

3.5 Different spot weld loading modes: In-plane and out-of-plane loading con-

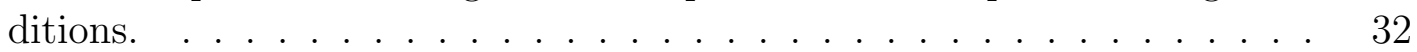

3.6 Critical zones of spot weld in a shear loading case for a High Strength

Steel (left) and a ductile steel (right). . . . . . . . . . . . . . . . . . 33

3.7 Stress distribution around weld notch. . . . . . . . . . . . . . . . . . . . 34

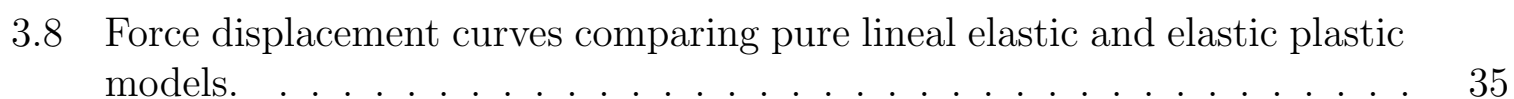

3.9 Crack tip coordinates and the domain where $J$-integral is evaluated. . . . $\quad 36$

$3.10 \quad J$-integral evaluation using multiple computational domain sizes. . . . . . 37

3.11 Strain fields around weld notch using different mesh sizes. . . . . . . . . 39 
3.12 Previous simulation loaded spot weld shear tests results at multiple meshes. 39

3.13 Numerical analysis of spot weld model of normal test. . . . . . . . . . . . 40

3.14 Ilustrative simulation results of loaded spot weld at multiple angles. . . . 42

$3.15 \quad J$-integral evolution at different sections. . . . . . . . . . . . . 43

4.1 Overview of the welding process cycle used in this work for the similar joints of 22MnB5 steel. . . . . . . . . . . . . . . . . . . . . . . . 49

4.2 Cross sectioned spot weld general view (I) . . . . . . . . . . . . . . 51

\begin{tabular}{|ll|l|l|}
\hline 4.3 & Detailed image of the weld notch of 22MnB5 similar joints of Figure & 4.2 ,
\end{tabular}

4.4 Cross sectioned spot weld general view (II). . . . . . . . . . . . . . 52

4.5 The|Vickers' hardness (HV) profile of a similar 22MnB5 steel joint (U15U15n). 53

4.6 An optical micrograph of the fully hardened 22MnB5 similar spot weld

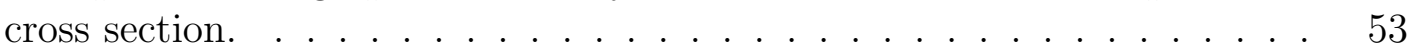

4.7 The optical micrographs of multiple spot weld zones of a similar 22MnB5

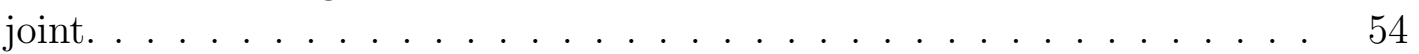

4.8 Reduced tensile tests specimens geometry. . . . . . . . . . . . . . . 55

4.9 Scheme of the process followed to obtain the micro specimens. . . . . . . 56

4.10 Reduced tensile 22MnB5 tests with and without weld material in the gauge

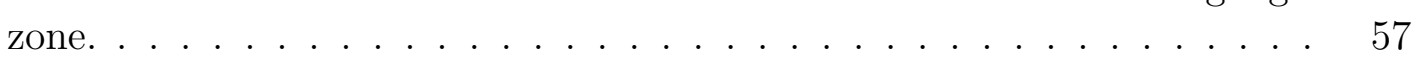

4.11 Force-displacement curves of shear tests for similar 22MnB5 joints. . . . 59

4.12 Force-displacement curves of normal tests for similar 22MnB5 joints. . . 60

4.13 Force-displacement curves of mix-mode tests for similar 22MnB5 joints and multiple thickness combinations. . . . . . . . . . . . . . . . 61

4.14 Force-displacement curves of tests with specimens of 22MnB5 with or without AlSi coating. . . . . . . . . . . . . . . 62

4.15 Force-displacements curves of shear tests with and without welding splashes. 62

4.16 Scheme of crack advance initiation in normal tests. . . . . . . . . . . . 63

4.17 Micro CT photo of cross sectioned weld of an interrupted normal test. A complex 3D crack propagation is observed. . . . . . . . . . . . . . 64

4.18 Differences in force-displacement curves of normal tests depending on crack front advance path. . . . . . . . . . . . . . . 64

4.19 Cross sectioned spot welds of 22MnB5 depending on sheet thickness ratio. 65

4.20 Cross-sectioned failed spot welds of 22MnB5 similar joints and corresponding failure mode. . . . . . . . . . . . . . 66

4.21 Simplified hardness profiles used for the hardness-based constitutive model of the welding zones. It is based on the experimental hardness profile presented in Figure 4.5 . . . . . . . . . . . . . . . . . . . 68

4.22 Simulation results of shear tests of 22MnB5 joints with the same thickness combination and multiple diameters. . . . . . . . . . . . . . 69

4.23 Simulation results of normal tests of $22 \mathrm{MnB} 5$ joints with multiple spot weld diameters. . . . . . . . . . . . . . . . . . . . . . . . . . . . . .

4.24 Front view of normal test. . . . . . . . . . . . . . . . . 71 
4.25 Simulation results for 22MnB5 joints of mix-mode tests with multiple spot weld sheet thickness combinations. . . . . . . . . . . . . . 71

4.26 A comparison of calculated and experimental critical forces of $22 \mathrm{MnB} 5$

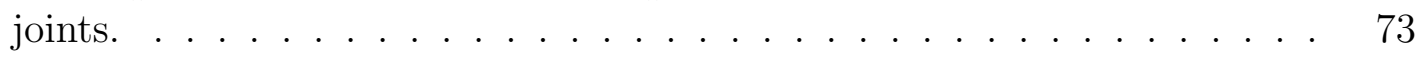

4.27 Maximum forces represented as a function of the weld diameter in the case of $22 \mathrm{MnB} 5$ joints. . . . . . . . . . . . . . . . . . 74

5.1 Cross sectioned spot weld (III). . . . . . . . . . . . . . . 77

$5.2 \quad$ Vickers hardness profile of a similar joint of DP980 steel. . . . . . . . . . 78

5.3 Optical micrographs of multiple spot weld zones of a DP 980 joint. . . . 79

5.4 Reduced tensile DP 980 tests with and without spot weld microstucture in the gauge zone. . . . . . . . . . . . . . . . . . . 81

\begin{tabular}{|l|l|l|}
\hline 5.5 & Finite element method (FEM) & model of the specimens used for the plas-
\end{tabular} ticity model calibration of the nugget material. . . . . . . . . . . 82

5.6 Hardening curves obtained from the force-displacement curves using the inverse FEM optimization scheme. . . . . . . . . . . . . . . . . . 83

$5.7 \quad$ Force-displacement curves of DP980 joints. . . . . . . . . . . . . . . . . . 84

5 Top view of failed spot welds at Full Interface Failure mode of DP 980

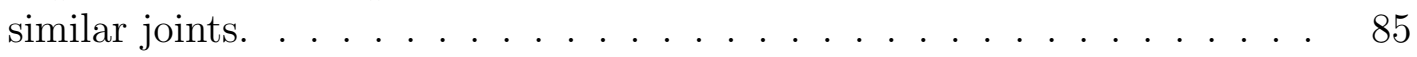

5.9 Cross sectioned failed spot welds of normal DP 980 similar joints. . . . . 86

5.10 Top view of failed spot welds of normal DP 980 similar joints. . . . . . . 86

5.11 Schematic representation of the welding zones under plasticity modeling point of view. . . . . . . . . . . . . . . . . . 87

5.12 Comparative between calculated and experimental force-displacement curves

of similar DP 980 joints. . . . . . . . . . . . . . . . . 88

5.13 Experimental and simulated results of normal tests for DP980 joints. . . 89

5.14 Maximum forces represented as a function of the weld diameter in case of DP980 shear tests. . . . . . . . . . . . . . . . . . . 90 90

6.1 Cross-sectioned spot weld general view (IV). . . . . . . . . . . . . . . . 93

6.2 Hardness measurements of DP 980 and 22MnB5 joints with different thicknesses' combinations. . . . . . . . . . . . . . . . . 94

$6.3 \quad$ Scheme of cross sectioned spot weld used for the nugget material charac-

\begin{tabular}{|l}
\hline terization of the dissimilar joints. $\ldots \ldots \ldots \ldots$ \\
\hline
\end{tabular}

$6.4 \quad$ Reduced tensile tests with spot weld microstucture in the gauge zone extracted from similar and dissimilar joints. . . . . . . . . . . . . . 96

6.5 Strain hardening curves of the weld nugget material for different steel grades combinations. . . . . . . . . . . . . . . . 97

$6.6 \quad$ Force-displacement curves of shear and mix-mode tests for dissimilar DP980 and 22 MnB5 joints. . . . . . . . . . . . . . . . . . . . 98

6.7 Force-displacement curves of normal tests of dissimilar joints. . . . . . . 99

6.8 Force-displacement curves of joints with same thickness combination, similar weld sizes and different material combinations. . . . . . . . . . . . . 100 
6.9 Cross-sectioned failure spot welds of dissimilar joints shear tests. . . . . . 101

6.10 Cross-sectioned failure spot welds of dissimilar joints normal tests. . . . . 102

6.11 Top view of failure spot welds of dissimilar joints normal tests. . . . . . . 102

6.12 Schematic representation of the welding zones of dissimilar joints under plasticity modeling point of view. . . . . . . . . . . . . . . . . 103

6.13 Comparative graph between force-displacement curves of dissimilar joints. 104

6.14 Experimental and simulated results of normal tests of dissimilar joints. . 105

6.15 Comparative graph between calculated and experimental critical forces of dissimilar joints. . . . . . . . . . . . . . . 106

6.16 Experimental and simulation forces at multiple diameters in dissimilar 22MnB5 and DP 980 joints. . . . . . . . . . . . . . . . . . 107

6.17 Simulation results of dissimilar shear tests depending on the method of obtaining the nugget hardening curve. . . . . . . . . . . . . . . 108

6.18 Simulation results of dissimilar normal tests depending on the method of obtaining the nugget hardening curve. . . . . . . . . . . . . . . . . . 109

7.1 $\quad$ Scheme of cross sectioned spot weld joining three sheets. These joints can be modeled as two separate joints. . . . . . . . . . . . . 113

7.2 Sketch of some welding irregularities permitted by the welding norm [1]. 115

7.3 Cross section of a spot weld extracted from a car body. . . . . . . . . . . 115

A.1 Crack front and $J$-integral coordinate system and arbitrary contour. . . . 122

A.2 Integral 3D domain around the notch tip. Surfaces, volume and $s$-function

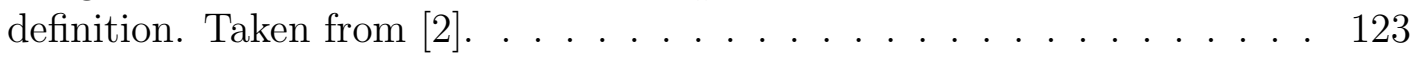

B.1 Obtained force displacement curves depending on type of boundary con-

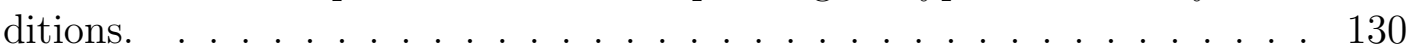




\section{Nomenclature}

The main symbols and acronyms used in this thesis are listed here. The symbols are clasified in Greek and Latin category.

\section{Latin symbols}

$\begin{array}{ll}a & \text { acceleration } \\ \mathbb{C}_{e l}^{\sigma J} & \text { elastic moduli } \\ \mathbb{C}_{e p} & \text { tangent elastic-plastic matrix } \\ D & \text { accumulated damage parameter } \\ \boldsymbol{D}^{e} & \text { elastic rate-of-deformation } \\ \boldsymbol{D}^{p} & \text { plastic rate-of-deformation } \\ \boldsymbol{D} & \text { total rate-of-deformation } \\ \boldsymbol{u} & \text { displacement field } \\ H V^{B M} & \text { base material hardness } \\ H V^{C H A Z} & \text { critical heat-affected zone hardness } \\ H V^{H H A Z} & \text { hard heat-affected zone hardness } \\ H V^{N} & \text { nugget hardness } \\ \boldsymbol{b} & \text { internal forces } \\ J^{e p} & \text { elastic-plastic } J \text {-integral } \\ J_{I C} & \text { critical } J \text {-integral for mode I fracture } \\ J_{I I C} & \text { critical } J \text {-integral for mode II fracture } \\ n_{F} & \text { Number of sample points } \\ r & \text { radial distance to resistance spot weld center } \\ r_{n} & \text { nugget radius }\end{array}$


$K \quad$ parameter of the Swift hardening law

$n \quad$ parameter of the Swift hardening law

$t \quad$ sheet thickness

$C_{1} \quad$ parameter of the Voce hardening law

$Q_{1} \quad$ parameter of the Voce hardening law

$\boldsymbol{v} \quad$ velocity field

$W \quad$ total strain energy density

$\boldsymbol{W} \quad$ spin tensor

$\boldsymbol{X} \quad$ Swift-Voce hardening parameter vector

$x_{D} \quad$ weighting parameter for the rule of mixtures

$Y \quad$ cost function for strain hardening parameter optimization

\section{Greek symbols}

$\beta \quad$ weighting factor for cost function $Y$

$\varepsilon^{e} \quad$ elastic strain tensor

$\varepsilon_{f} \quad$ equivalent plastic strain to fracture

$\bar{\varepsilon}_{p} \quad$ effective plastic strain

$\varepsilon \quad$ total strain tensor

$\rho_{0} \quad$ original density

$\boldsymbol{\sigma}^{\boldsymbol{D}} \quad$ deviatoric stress tensor

$\sigma_{e q} \quad$ equivalent von Mises stress

$\sigma_{k} \quad$ yield stress

$\boldsymbol{\sigma}^{\nabla J}$ rate of the Cauchy stress

$\boldsymbol{\sigma}$ Cauchy stress tensor

$\varepsilon_{0} \quad$ parameter of the Swift hardening law

$\alpha \quad$ mixing parameter

$\sigma_{0} \quad$ parameter of the Voce hardening law 


\section{Acronyms}

\begin{tabular}{|c|c|}
\hline AHSS & advanced high strength steels \\
\hline $\mathrm{BiW}$ & Body-in-White \\
\hline $\mathrm{BM}$ & base material \\
\hline $\mathrm{BPO}$ & button pullout \\
\hline CGHAZ & coarse grain HAZ \\
\hline $\mathrm{CT}$ & cross tension \\
\hline DIC & digital image correlation \\
\hline EDM & electro discharge machining \\
\hline FEM & finite element method \\
\hline FGHAZ & fine grain HAZ \\
\hline FIF & full interface failure \\
\hline FZ & fusion zone \\
\hline HAZ & heat-affected zone \\
\hline HHAZ & hard HAZ \\
\hline $\mathrm{HV}$ & Vickers' hardness \\
\hline LEFM & linear elastic fracture mechanics \\
\hline MMM & modular material model \\
\hline PDF & partial dome failure \\
\hline $\mathrm{PIF}$ & partial interface failure \\
\hline PST & plane strain tension \\
\hline
\end{tabular}


SHAZ soft HAZ

SW spot weld

TDF total dome failure

TS tensile shear

UT uniaxial tension

UTS ultimate tensile strength

VPS Virtual Performance Solution 


\section{Chapter 1}

\section{Introduction}

\subsection{Motivation}

The automotive industry is constantly involved in the development of new projects aimed at reducing weight, fuel consumptions and costs. To achieve these safety requirements, new materials such as the so-called AHSS are being introduced to new car structures. These steels exhibit superior material properties as compared to conventional steels, with ultimate tensile strength (UTS) of more than $750 \mathrm{MPa}$ [3], they allow weight reduction while maintaining or even improving passenger safety. With the increase in the bearing capacity of crash-relevant structural components, the response of joints achieved by techniques such as adhesive bonding and resistance spot welding (RSW) become critical.

Nowadays, RSW is the most widely used sheet metal joining technique in the automotive industry. A car's structure typically presents more than five thousand spot welds(SWs), Among them, more than two thousand involve at least one AHSS sheet. RSW is a thermo-electric process that consists on generating heat at the interface of the parts being welded by passing an electrical current through them under a controlled pressure. As a result, this process induces changes in the microstructure and hence the mechanical properties. The welding process leads to two distinct zones: the fusion zone (FZ) or nugget and the so-called heat-affected zone (HAZ). The final bearing capacity of the welded joints is mainly determined by the resulting weld geometry and the resulting mechanical properties' distribution, both depending on the material composition of the joined sheets and the welding process itself [4]. With 
the introduction of AHSS, the improved mechanical properties of the base material metal sheets lead to a new mechanical property distribution around the weld, making to arise different failure mechanisms in the SWs not observed for conventional ductile steels [5, 6].

Furthermore, during the development of new car projects, full-vehicle models based on the FEM are used to perform crash simulations to assess the structure integrity as well as occupants and pedestrian safety. The car structural components are normally modeled using a 5-8 mm shell elements mesh. The mechanical response of the SWs that join these components is simulated with simplified models to avoid unaffordable computational costs. Briefly, the current procedures to predict SWs failure in full-vehicle crash simulations use over-simplistic rigid beam models yet giving reasonable results for standard ductile steels, mainly because failures usually occur at the sheets base material and not in the joints [7 9]. However, these models fail to predict the fracture of the SWs in AHSS due to the inability to accurate model the specific failure mechanisms occurring in the spot welds [6, 10, 11]. Consequently, these models were improved by introducing the maximum forces at the spot welds obtained from extensive experimental characterization campaigns. However, these experimental campaigns are extremely time and money consuming.

A comprehensive analysis of the resistance spot welds failure mechanisms in AHSS considering multiple loading conditions, material, and thickness combinations would improve the current models and likely reduces the experimental campaigns. For this purpose, detailed FEM models of SWs have been developed during the last few years and tested in isolated components [12 17]. These models reproduce in detail the mechanical response of a joined specimen during different loading conditions, considering the inhomogeneities of the $\mathrm{SW}$ material and using a suited fracture criterion. However, these models are not extensively used in the industry due to long-term characterization campaigns that are needed for their calibration that must be done each time the joints of a new steel grade have to be characterized.

\subsection{Objectives}

The main objective of this thesis is twofold: (1) to develop a detailed FEM model that is able to partially replace the extensive experimental campaign in providing parameters for the crash simulations, and (2) to gain understanding of the loading 
and fracture responses of SWS in AHSS in order to improve a simplified model yet beyond current models that could be incorporated in full-vehicle crash simulations with thousands of spot welds.

The model resulting from (1) must be applicable to multiple sheet material and thickness combinations and a wide range of loading conditions, all relevant to fullvehicle simulations. It is generally known that the fracture response of the SWS strongly depends on the weld size for some loading conditions [4, 17]. Special attention will therefore be devoted to this feature. Additional factors such as the side effects of the welding process (presence of inclusions, porosities, residual stresses...), the metal sheet coating, and the loading velocities are considered of low relevance in the final fracture response and are not further analyzed in this thesis.

Previous research works show that failure of SWs at AHSSs under in-plane loading conditions starts in the so-called softened HAZ with lower material properties [6, 10, 18]. However, under out-of-plane loading conditions the onset of crack is located at the weld notch due to the stress concentration in this singular zone that further loaded results in the nugget fracture [6, 11, 17]. The main focus of this thesis is the simple and robust modeling of fracture of spot welds under out-of-plane loading conditions in AHSS joints. The proposed approach should minimize as much as possible the computational costs as well as complex numerical implementations no suited for industrial purposes. A complete detailed characterization of the different weld zones mechanical properties is beyond the scope of this thesis. However, a reduced material characterization procedure will be followed if necessary for completing the spot weld constitutive model.

\subsection{Work approach}

A FEM model for the prediction of SW failure in AHSS will be developed in this thesis. As mentioned previously, the mechanical response of spot welded joints is determined mainly by the geometry and the material properties resulting from the welding process. Therefore, the mechanical characterization of the weld material as well as the definition of the weld geometry are necessary. Furthermore, an appropriated fracture criterion that assesses the intensity of weld notch stress field (i.e. the failure trigger) is applied. The results obtained with this detailed FEM model are validated with a large experimental campaign of loading tests of welded specimens 
that considers most of the main factors that have an influence on fracture response, i.e. sheet thickness and material combinations, weld sizes and loading angles. Combinations of steels of two AHSS grades are considered in this thesis. These steels are a martensitic boron steeel (the so-called 22MnB5) and a dual phase steel (the so-called DP 980).

The proposed work approach is sketched in Figure 1.1. The same procedure is followed for the three material combinations analyzed in this thesis. Experimental loading tests of the welded specimens are performed and the results are compared with the results obtained from the detailed FEM model.

To define the spot weld model for a certain material sheets combination, several spot welds are cross-sectioned in other to obtain the geometry, and perform a microscope optical observations and $\mathrm{HV}$ lines. On one side, the geometry parameters are used in the detailed spot weld model. On the other side, from both the obtained hardness and the metallography analysis, the most important welding zones regarding material properties are distinguished. Material characterization of those zones with no available data is carried out. Special micro-tensile tests are performed and using an inverse FEM iterative optimization procedure the plasticity model of the weld zones is fitted to the micro-tensile test force-displacement curves previously gathered.

Based on previous preliminary simulations as well as experimental and simulation works [10, 17, 19, a fracture criterion based on the $J$-integral is selected as the best suited for the maximum force prediction of this kind of joints under out-ofplane loading conditions. The $J$-integral is evaluated gathering the results of the FEM simulation of the loading tests. A quantitative comparison between simulation and experiment is conducted based on one validation metric: the maximum bearing force. Experimentally obtained and simulated force-displacement curves have also been compared qualitatively for validation purposes. The $J$-integral criterion can predict fracture only in those cases in which it stars from the weld notch. A detailed analysis of the fracture behavior of the experimental loading tests is also given in this thesis to confirm this assumption. 
1.4. Chapter overview

\section{RSW}

cross-section

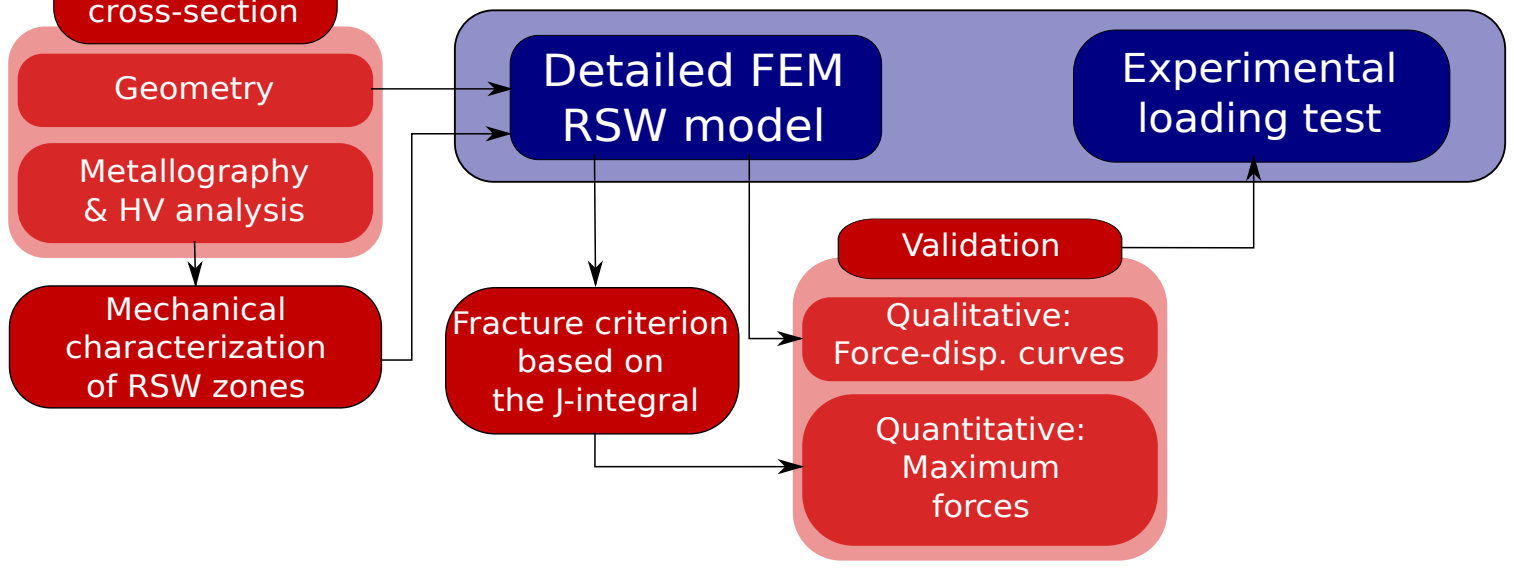

Figure 1.1: Proposed work approach covering the analysis for multiple combinations of loads, material, and sheets' thicknesses.

\subsection{Chapter overview}

An introduction to the main related topics with the thesis as well as the state of the art in spot weld experimental failure characterization and simulation models are presented in Chapter 2. It includes a short introduction to the car safety performance assessment tests in Section 2.1, a description of AHSS in Section 2.2, an explanation about the RSW process in Section 2.3, and the description of the experimental loading tests used for the characterization of joints' bearing capacity in Section 2.4. The state-of-the-art of the modeling of spot weld loading and fracture response is also introduced in Section 2.5, where the simplified models used for the full-vehicle crash simulations are introduced and the detailed models that reproduce the experimental loading tests of isolated joined specimens are presented. This chapter ends with a review of the different current approaches to the modeling of fracture in spot welds.

The SW FEM model used in this thesis is presented in Chapter 3. This model is validated in the next chapters using experimental loading tests.

A description of both the solved goberning equation as well as the constitutive model is presented in Section 3.1 and 3.2, respectively. The definition of the mechanical properties heterogeneities of the weld is presented in Section 3.3. The reasons for the selection of the $J$-integral as fracture criterion is presented in Section 3.6. A detailed explanation of this criterion is depicted. Some issues of the numerical model 
are analyzed in Section 3.7 taking into account the constraints of low computational costs and use of a commercial explicit code. Some illustrative results of the SW FEM model are presented in Section 3.8 using previous available data for 22MnB5 joints in terms of material zones characterization.

The SW FEM model is applied to experimental tests of the "similar joints" of 22MnB5 hot formed steel in Chapter 4. An experimental loading test campaign to validate the model is presented in Section 4.1. The test matrix considers multiple sheet thickness combinations, loading angles and different weld diameters obtained using multiple sets of welding parameters. The geometry and the local material properties distribution are analyzed in Section 4.2 using cross-sectioned spot welds of all combinations of the test matrix. The strain hardening curves of the nugget fusion zone were not available, consequently in order to confirm the assumption that the mechanical properties of the nugget are the same as the base material, microtensile tests of raw material and welded material are carried out in Section 4.3. The experimental loading tests of the joining specimen are presented in Section 4.4. Lastly, using all the detailed characterizations extracted from the previous sections, the spot weld model for these types of joints is validated in Section 4.5 using the results obtained from the experimental tests.

The model is applied for "similar joints" of DP 980 material in Chapter 5 . A brief description of the experimental campaign is given in Section 5.1. The same procedure followed in the previous chapter to define the geometry and the local material properties distribution is given in Section 5.2. The parameters of the plasticity model of the nugget material of this type of joints are not available. Thus a characterization campaign using micro-tensile tests and finite element based parameters identification has been conducted as described in Section 5.3. The experimental loading tests of the joining specimen can be found in Section 5.4 . The results are compared in Section 5.5 with simulated results in order to validate the spot weld model.

A similar procedure is used in Chapter 6 for dissimilar joints of $22 \mathrm{MnB} 5$ and DP 980 sheets, where the experimental campaign is presented in Section 6.1, the geometry and the metallographic analysis is given in Section 6.2, and the characterization of the nugget material is introduced in Section 6.3. The experimental loading tests results are presented in Section 6.4. The validation of the model using the simulated and experimental resuls is presented in Section 6.5.

Lastly, some hints for the application of the model for other spot welds that can 
be found in a full-vehicle appart from those analyzed in the previous chapters are presented in Chapter 7. It includes other sheet combinations in Section 7.1, different loading conditions in Section 7.2. In Section 7.3, the main differences that can be found in real vehicle industrial welds when they are compared with those obtained in laboratory conditions, like those analyzed in this thesis, are pointed out.

In Chapter 8 are presented a detailed summary of this thesis and the more relevant conclusions. 



\section{Chapter 2}

\section{State of the art}

A short introduction and theoretical background of the topics related with the current thesis is presented in this chapter. It includes an introduction to the vehicle crash tests in Section 2.1, as well as a description of the AHSSs and the steel grades used in the thesis in Section 2.2. Some details about the RSW process, as well as the properties and geometry resulted from it depending on the joined steel grades are introduced in Section 2.3, and finally the loading tests used to characterize the bearing capacity of the joints are presented in Section 2.4.

Various attempts to model spot weld failure have been made for a long time. Two different approaches have been followed for this purpose. On the one hand, simplified models are used in full-vehicle crash models and are fitted by using maximum forces obtained from experimental tests. On the other hand, detailed FEM models reproduce the spot weld response under some certain loading conditions in order to obtain the joints bearing forces. These detailed models includes the spot weld heterogeneous material properties and geometry along with a suited fracture criterion. Both types of spot welds modeling are described in detail in Section 2.5. Finally, this thesis is focused on the detailed FEM models using an appropriate fracture criterion to predict spot weld bearing capacity. For this purpose, several approaches used in the literature to predict metal sheet's joints fracture are presented and analyzed. 


\section{$2.1 \quad$ Vehicle crash tests}

The safety performance of a vehicle is assessed by passing a certain series of regulations, consumer tests assessments and likely internal requirements. For instance, in the case of European Union, vehicles are tested using Euro NCAP protocols. Front, side and rear impact crash tests are carried out in order to asses the crash structure integrity and occupants safety.

A comprehensive review of the previously defined project targets concerning car structure integrity and passenger and pedestrians safety during the prototype phase entail high costs due to the nature of tests and the prototypes themselves. To reduce the number of crashed cars, full vehicle finite element models are used (see an example of a frontal crash model in Figure 2.1). In these models, the car structure is modeled using shell type elements with a element size of around $5 \mathrm{~mm}$ providing high prediction quality in terms of deformations. However, these simulations entail high computational costs due to the great amount of computed parts. For instance, to calculate $140 \mathrm{~ms}$ of a crash situation (the inital time where most relevant structural events occor) more than 640 hours of CPU time are needed using the current models.

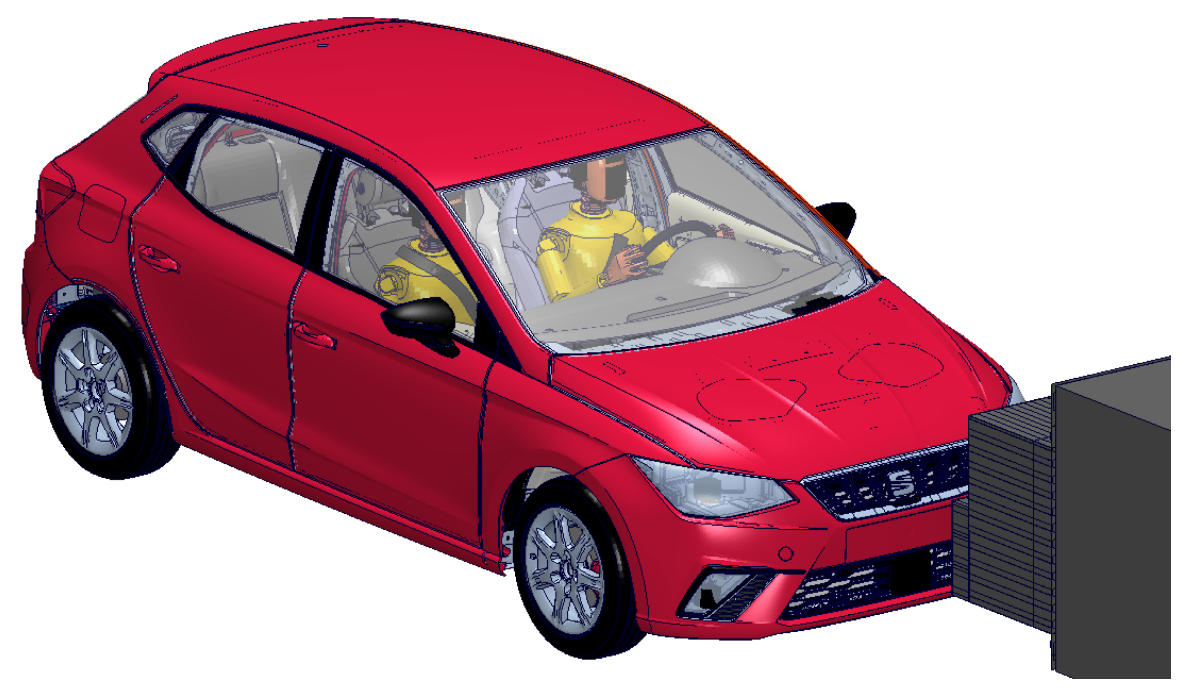

Figure 2.1: Full vehicle model of an Euro NCAP frontal crash. Source: SEAT, S. A. 


\subsection{The Advanced High Strength Steels family}

Regarding the development of vehicle structures, one of the most important features is a safety cell that avoids external intrusions during crash to protect the cars passengers. For this reason, so-called AHSSs have been introduced in vehicle structural components. Various research studies have shown that proper application of AHSS can reduce a vehicles weight between 10 and $25 \%$ [20]. The family of AHSSs are defined by those steels with tensile strengths exceeding $780 \mathrm{MPa}$ [3, 21]. These extraordinary properties are achieved by different microstructures; conventional mild steels (also known as ductile steels) have single-phase ferritic microstructure, however the AHSSs have more complex microstucture that gives them a better performance. This excellent mechanical performance of AHSS grades results in a high proportion of this type of materials in the $\mathrm{BiW}$ of current vehicles. A modern car structure is presented in Figure 2.2, most of the structural relevant components are fabricated in two of the most used AHSS grades, the hot formed martensitic steels and the cold formed dual phase steels.

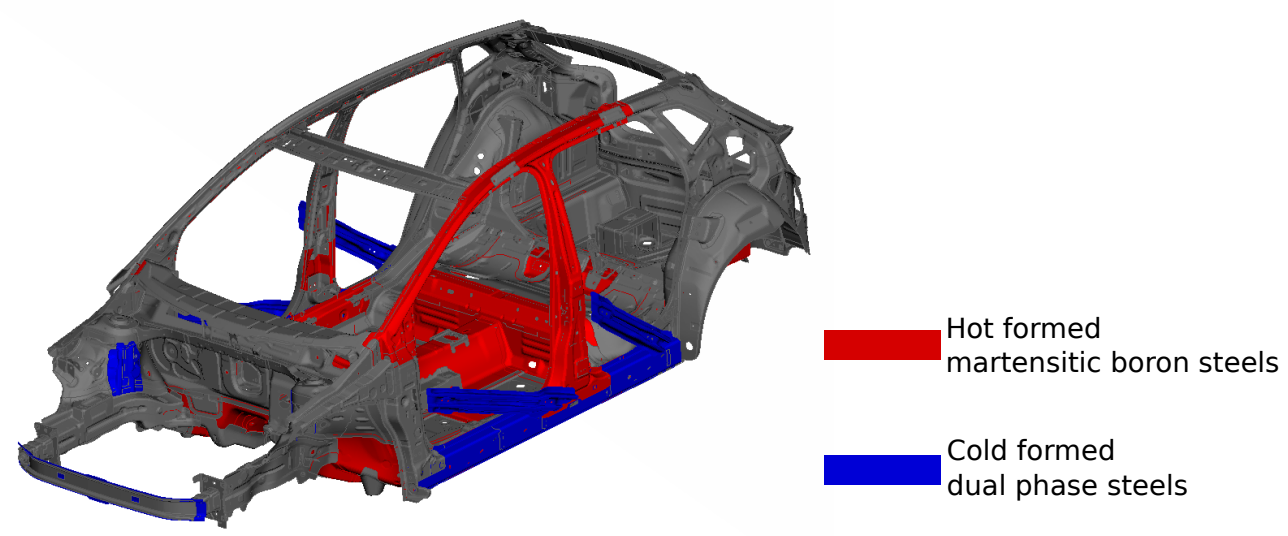

Figure 2.2: $\mathrm{BiW}$ of one modern vehicle. A high proportion of hot formed martensitic and dual phase steels (both included in AHSS family) is observed. Source: SEAT, S.A.

In this thesis, the joints of these two aforementioned steel grades are analyzed: On one side, martensitic steel known as 22MnB5 and on the other side dual phase steel known as DP 980. 22MnB5 belongs to the hot formed martensitic manganeneseboron steels $(\mathrm{MnB}+\mathrm{HF})$ and DP 980 to dual phase steels (DP). Figure 2.3 shows the global formability diagram used to classify the steel grades. The higher ultimate 
strength of martensitic steels can be observed. On the contrary, dual phase steels are associated with a higher maximum elongation, and lower tensile strength. However, it must be pointed out, than some authors have recently proposed that, instead of ultimate tensile strength and maximum elongation, the crashworthiness of the AHSS should be assessed by means of energetic parameters, such as fracture toughness or the essential work of fracture [22]. The approach presented in this thesis for spot weld fracture modeling is in accordance with the steel characterization recently proposed.

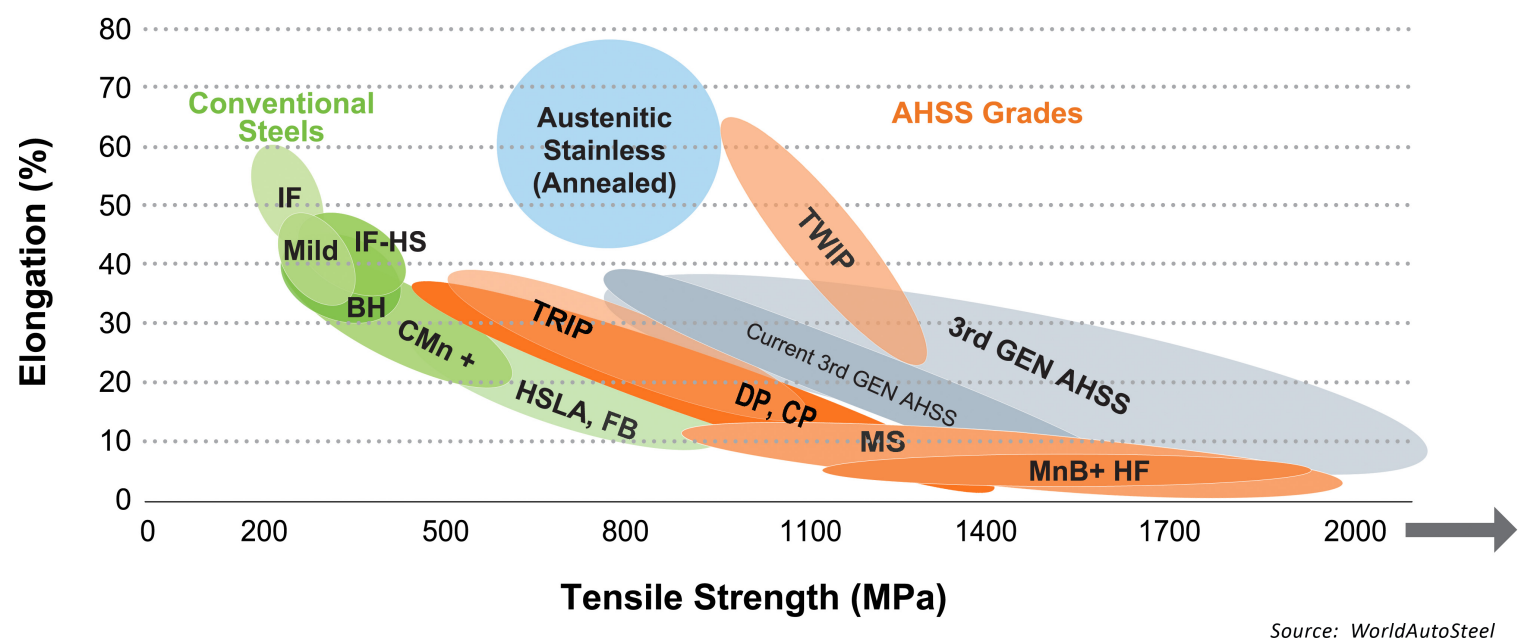

Figure 2.3: Global formability diagram for todays's AHSS grades. Source: [23].

The first analyzed steel grade in this thesis is the 22MnB5, a hot forming boron steel with martensitic microstructure. This steel is delivered as a ferritic/pearlitic ductile microstructure, and during the press hardening process and quenching in cooled stamping tools it is transformed into a fully martensitic brittle and high strength microstructure. With this hot forming process the ultimate tensile strength reaches values of around $1500 \mathrm{MPa}$ with and maximum elongation decreases up to $6 \%$ [24]. The chemical composition of this steel can be seen in Table 2.2. It is delivered with aluminum-silicon coating (the so-called AlSi coating) that protects it against oxidation and decarburation during hot forming process and that has also an influence on the weldability. The final material microstucture and consequently the mechanical properties depend on the press hardening process. In this work, only the steel with full martensitic microstructure, also known as full hardened 22MnB5, is considered. 
2.3. Resistance spot welding

\begin{tabular}{cccccccc}
\hline $\mathrm{C}$ & $\mathrm{Mn}$ & $\mathrm{P}$ & $\mathrm{S}$ & $\mathrm{Si}$ & $\mathrm{Al}$ & $\mathrm{Ti}$ & $\mathrm{B}$ \\
$0.2-0.25$ & $1.1-1.4$ & $\leq 0.025$ & $\leq 0.008$ & $0.15-0.35$ & $\geq 0.015$ & $0.02-0.05$ & $0.002-0.005$ \\
\hline
\end{tabular}

Table 2.1: Chemical composition of 22MnB5 used in this work (wt. \%) 24].

The second analyzed steel is the DP 980, also known HCT980X under the DIN EN standard. It is a dual phase steel consisting of a ferrite matrix containing a hard second phase of islands of martensite. This microstructure gives it an ultimate tensile strength of around $980 \mathrm{MPa}$ and an $11 \%$ of maximum elongation [25]. Although the ultimate tensile strength of $0.98 \mathrm{GPa}$ is lower than $22 \mathrm{MnB} 5$ (UTS=1.5 GPa), the cold forming process is profitable for the industry due to the lower production costs. This steel is supplied with the chemical composition depicted in Table 2.2 and with a zinc coating. The structural components of this steel are achieved using a cold forming process. This process may introduce the so-called spring back phenomenon. Spring back occurs when the material tries to return to its original shape after being bent, consequently the desired finally geometries of the workpieces are not accurately reached.

\begin{tabular}{ccccccccc}
\hline $\mathrm{C}$ & $\mathrm{Mn}$ & $\mathrm{P}$ & $\mathrm{S}$ & $\mathrm{Si}$ & $\mathrm{Al}$ & $\mathrm{Ti}+\mathrm{Nb}$ & $\mathrm{B}$ & $\mathrm{Cr}+\mathrm{Mo}$ \\
$\leq 0.2$ & $\leq 2.90$ & $\leq 0.080$ & $\leq 0.015$ & $\leq 1.00$ & $0.015-2.0$ & $\leq 0.15$ & $\leq 0.005$ & $\leq 1,00$ \\
\hline
\end{tabular}

Table 2.2: Chemical composition of DP980 used in this work (wt. \%) [25].

\subsection{Resistance spot welding}

The higher bearing capacity of the crash-relevant structural components leads to higher relevance of the joints. $\mathrm{RSW}$ is the most important joining technique and consequently it is crucial to know the loading and fracture response, i.e. the maximum forces of joints, during vehicle crash situations as presented in Chapter 1 .

A scheme of the RSW process is presented in Figure 2.4. It involves three stages: (i) A force is applied to two or three metal sheets using two copper electrodes during the so-called "squeeze time", (ii) an electrical current is applied with the electrodes while pressure is maintained during the so-called "weld time", (iii) finally the current is removed and the electrodes remain in place for the material to cool during the 
so-called "forge time". The electrical current produces heat in the interface of the parts being welded due to Joule effect and leading to material melting. After cooling and material solidification both sheets stay joined. The process total time ranges from 1 to 2 seconds depending on the thickness of the sheets. The electrical current can be applied in one or two pulses.

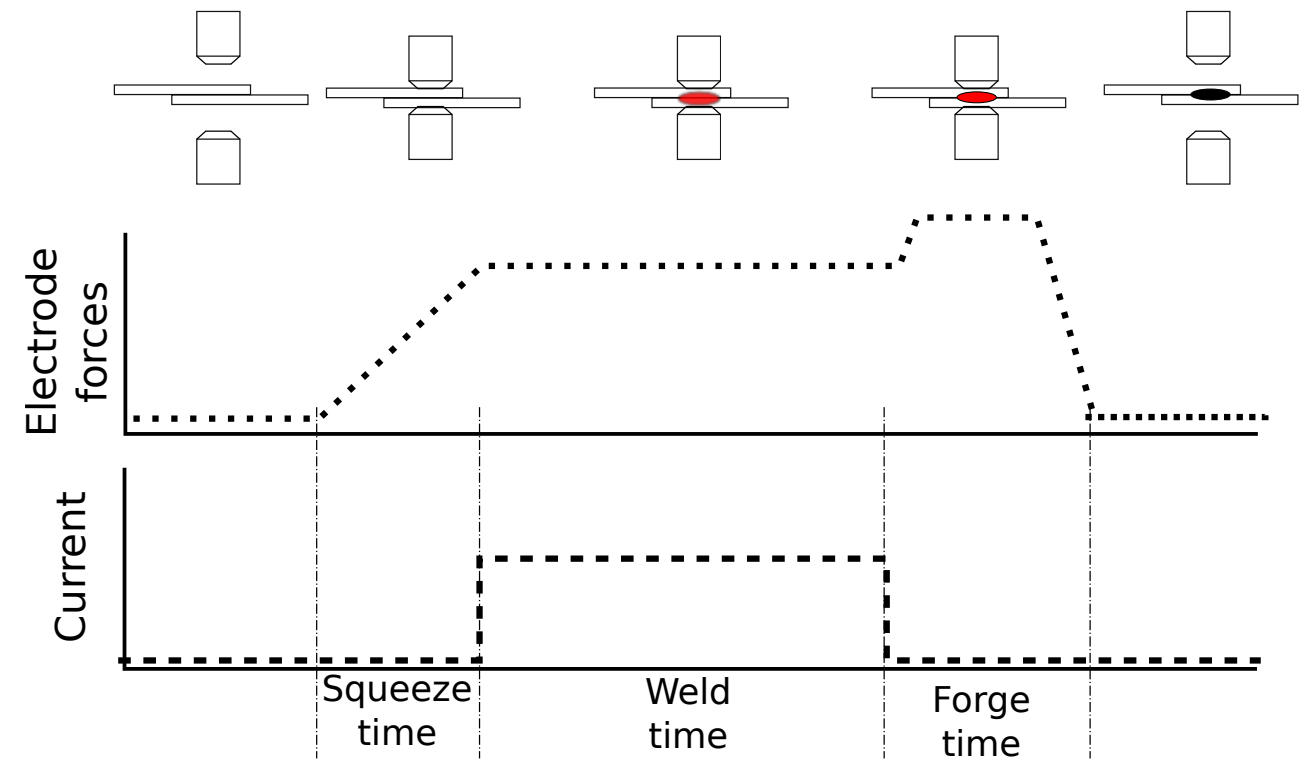

Figure 2.4: Schematic representation of resistance spot welding process.

As previously presented, this thermo-mechanical process introduces in the joint mechanical properties changes, geometrical features and imperfections that determine the loading and fracture response of the spot welds. The thermal evolution around the weld induces microstructure changes on the material that leads to a different material properties distribution. Three main zones can be distinguished in a $[\mathrm{SW}$ as can be observed in the scheme of Figure 2.5. (i) The base material (BM), whose properties are not modified due to the welding process, (ii) the FZ or nugget, where the material is melted during the welding process, and (iii) the HAZ, in which the microstucture and therefore the material properties are modified due to the different thermal cycles with decreasing peak temperature. The HAZ is situated between BM and FZ.

The spot weld miscrostrucutre distribution of multiple AHSS grades has been deeply analyzed in [4, 26, 29]. The microstructure distribution and consequently mechanical properties are usually characterized using hardness profile measurements 
using indentation lines crossing the spot weld. The hardness profiles schemes of multiple steels ranges are presented in Figure 2.5. It is observed, that the hardness of the FZ has high values in all steel grades. They are a consequence of the untempered martensitic microstructure resulting from the rapid cooling produced by the welding process. For most of the steels, a martensitic hard HAZ (HHAZ) close to the FZ can be found. This region experiences peak temperatures above $\mathrm{Ac}_{3}$ without reaching the melting temperature transforming $\mathrm{BM}$ microstructure into austenite that is transformed during cooling into martensite. The microstucture of region between this HHAZ and the BM depends on the joined steeels composition and is given by the different phase transformation due to the thermal evolution. Different hardness values are observed for BM depending on the steel. Martensitic steels present high hardness values and conventional steels low values in this zone [4]. During welding, the martensitic base material is reheated leading to softer microstructures in the socalled soft HAZ (SHAZ). Notice that this SHAZ cannot be found in the conventional steels, where BM has low hardness values itself.

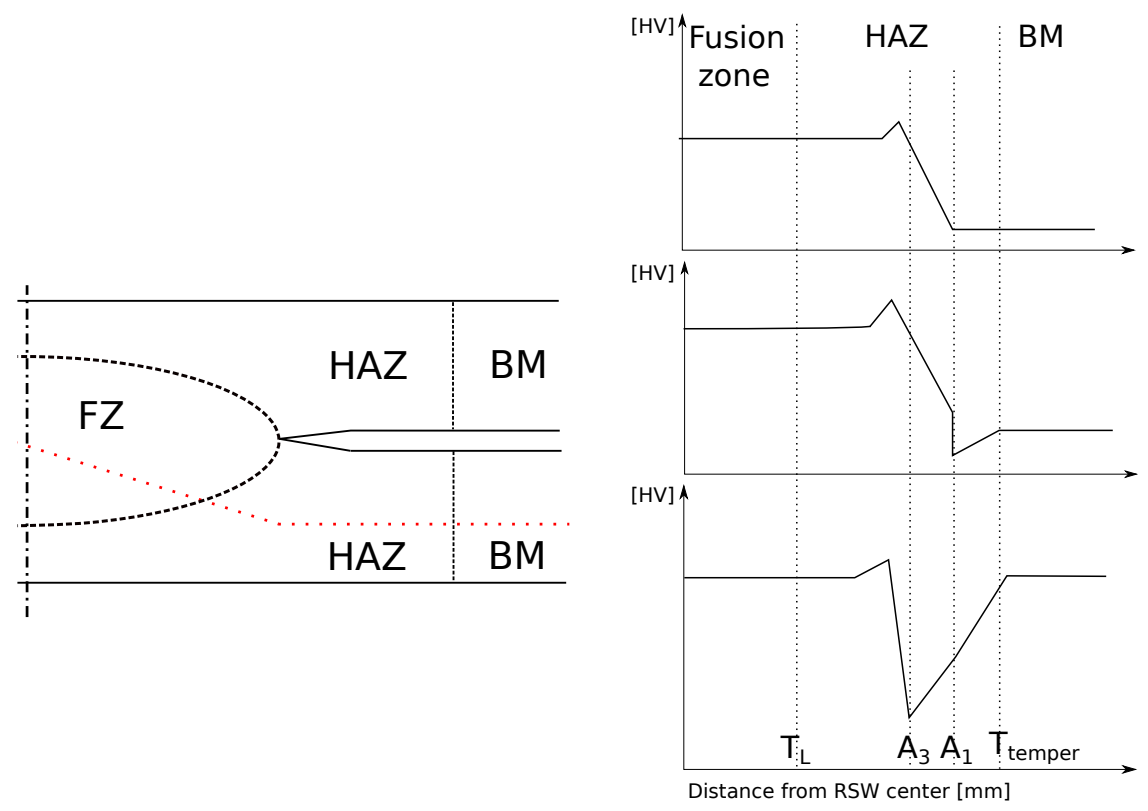

Figure 2.5: Typical hardness profile of resistance spot welds of different steel grades. From top to bottom: (i) conventional ductile steel and steels without HAZ softening, (ii) dual phase steels which experiences HAZ softening (e.g. DP780 and DP980), and (iii) martensitic steels [4]. Notice that the hardness profiles are subdivided in different zones related with the peak temperatures reached during the welding process.

The welding process introduces not only material properties changes zones but 
also some geometrical features that would determine the spot weld loading response [4]. The main geometrical features of a spot weld can be seen in Figure 2.6, and are detailed as follows :

- Spot weld size: It depends mainly, but not only, on the set of welding parameters, such as applied force, currents and times. The nugget or fusion zone has an oblate spheroid shape. When a spot weld is cross-sectioned, it appears as an ellipse-like geometry called fusion line. The nugget diameter, that is defined as the maximum axis of this ellipsis, is used normally to define the spot weld size (see Figure 2.6). The faying surface is defined as the intersection between the nugget spheroid and the sheets interface plane. The faying surface is parallel to the sheets plane and has a circle shape. This circle is parametrized using the so-called joining diameter, which is the same as nugget diameter in the case of joints of both sheets of the same thickness but not for other combinations.

- Weld notch: It is a notch generated after the welding process. It refers to a geometry with notch-like shape that is generated around the weld where both sheets converge. Some authors postulate that the weld notch shape, sharp or square, can affect the failure response of the spot welds [30]. Two notches can be observed in both nugget extremes in the cross-sections but it is actually an unique crack front with a circle shape surrounding the nugget. For coated materials, such as $22 \mathrm{MnB} 5$, it has a very irregular geometry due to the presence of the remaining coating [31].

- Weld penetration depth: It is the depth that the nugget penetrates into each one of the sheets. If it is not enough the joints are not considered valid.

- Electrode indentation depth: It is the depth that electrodes forces generate in both sides of the joint. It may have an influence on the joint failure response just in case of very thin sheets (less than $0.8 \mathrm{~mm}$ ).

Furthermore, the welding process leads sometimes to undesirable welding imperfections, which would have an influence on the RSW failure response, reducing the maximum bearing forces. The most important imperfections are the welding voids in the nugget and the welding splashes. Welding voids and porosities (see Figure 2.7) are consequence of the shrinkage phenomenon during the solidification process, mainly 


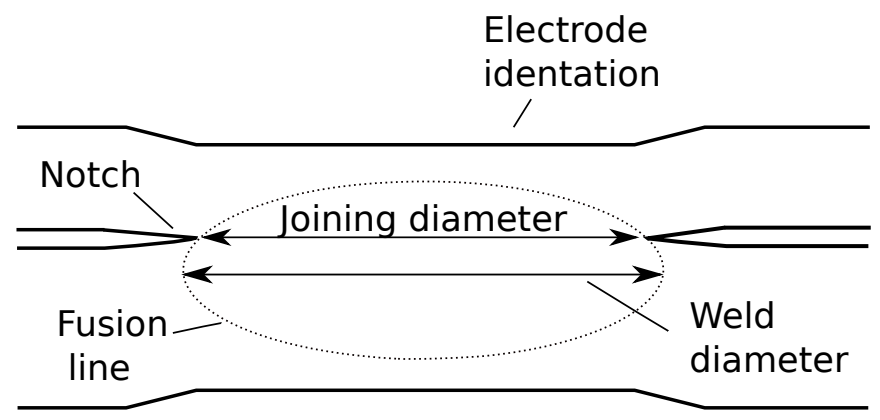

Figure 2.6: Schematic resistance spot weld cross sections with the main geometrical features [5, 6, 17].

due insufficient electrode applied load [4]. Furthermore, the welding splashes or expulsions may decrease the desired weld diameter, due to the expulsion of material. They are caused mainly by high welding currents and low applied forces. These phenomena along with other imperfections such as inclusions, or micro-cracks are very difficult to control, because they do not depend exclusively on the welding parameters but also on uncontrollable phenomena, such as the atmosphere conditions (mainly humidity and temperature) or electrode cap state.

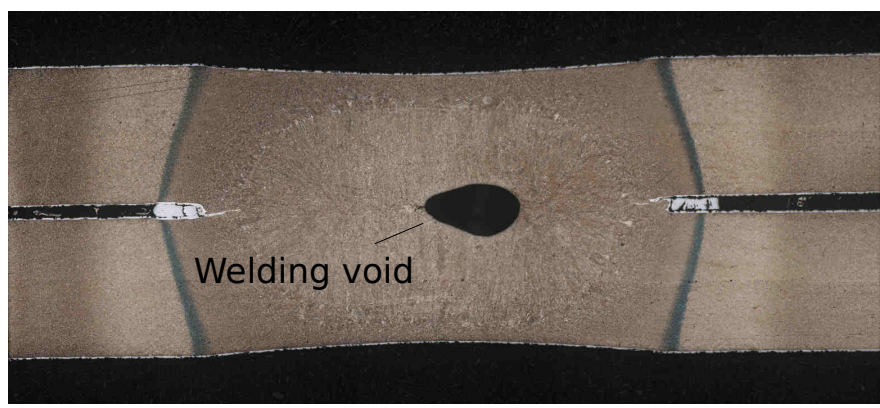

Figure 2.7: Cross-sectioned spot weld with a welding void or pore.

\subsection{Experimental characterization of spot weld failure}

Due to the complexity of spot welds, the joints loading and fracture response have been deeply analyzed in many previous works [10, 16, 32 35]. Two loading test are usually carried out in order to assess the fracture and load response. They are the 
"cross tension test" and "tensile shear test" (see Figure 2.8). These tests need coupons with simple sheet rectangular geometries and are used to evaluate joint maximum pure normal forces in the case of cross tension (CT) test and pure shear forces regarding tensile shear (TS) test. Some new specimens and tests have been used during the past years to characterize the joint in more complex loading conditions. One example is the KS-II specimen, that is used to load the joint at different angles [36]. The name of the loading angle is given by the angle between the applied force direction and the sheet plane. Apart from zero degrees tests (equivalent to TS test) and ninety degrees test (equivalent to $\mathrm{CT}$ test), the joint can be loaded at sixty and thirty degrees.
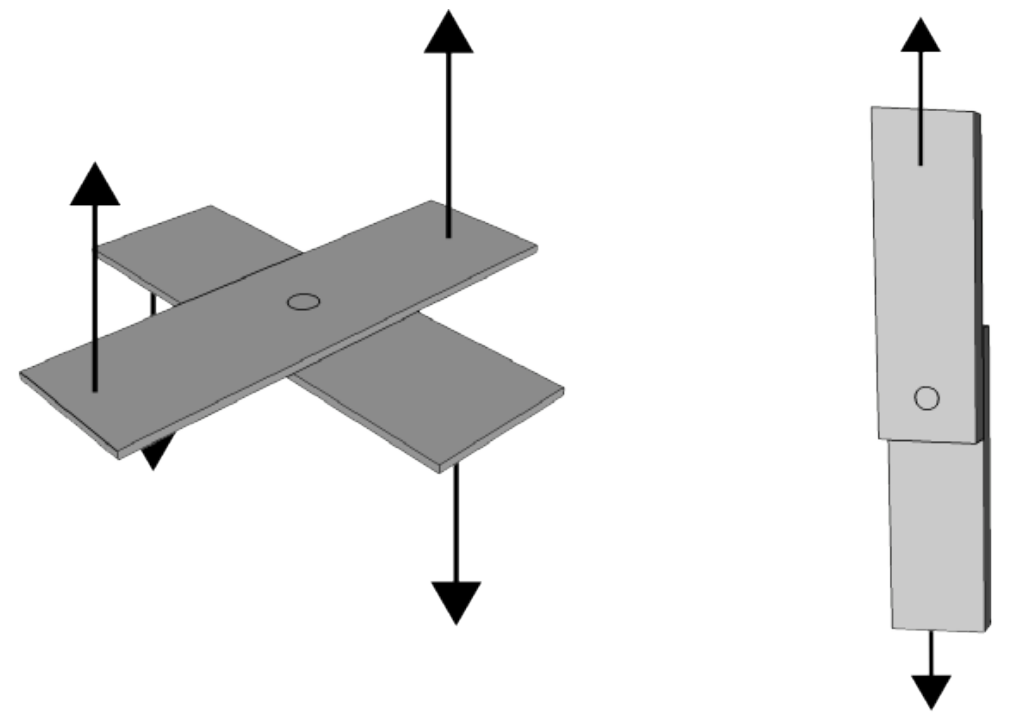

Figure 2.8: Sketch of the Cross Tension (left) and Tensile Shear (right) tests.

Using these tests, the fracture response of a high number of steels ranges, sheets' thickness combinations, and sets of welding parameters have been analyzed in previous works. Different failures modes are usually identified when referring the failed spot weld appearance [4]. The typical failure modes for spot welds in conventional steels based are [37]: (i) button pullout (BPO) in BM, and (ii) full interface failure (FIF) or partial interface failure (PIF) [4, 33, 35, 38].

In joints of AHSS s sheets the aforementioned inhomogeneous material properties distribution introduces new failure mechanisms: (i) failure due to necking in SHAZ, and (ii) failure due to ductile shear in the FZ and HAZ boundary [6]. Fracture in SHAZ leads to failed welds with BPO failure mode appearance (see BPO-SHAZ in 
Figure 2.9). The failure mechanism along the boundary of the nugget leads in some conditions to a new failure mode the so-called total dome failure (TDF) or partial dome failure (PDF), usually found in $22 \mathrm{MnB} 5$ joints [11. A scheme of all failure modes can be seen in Figure 2.9.
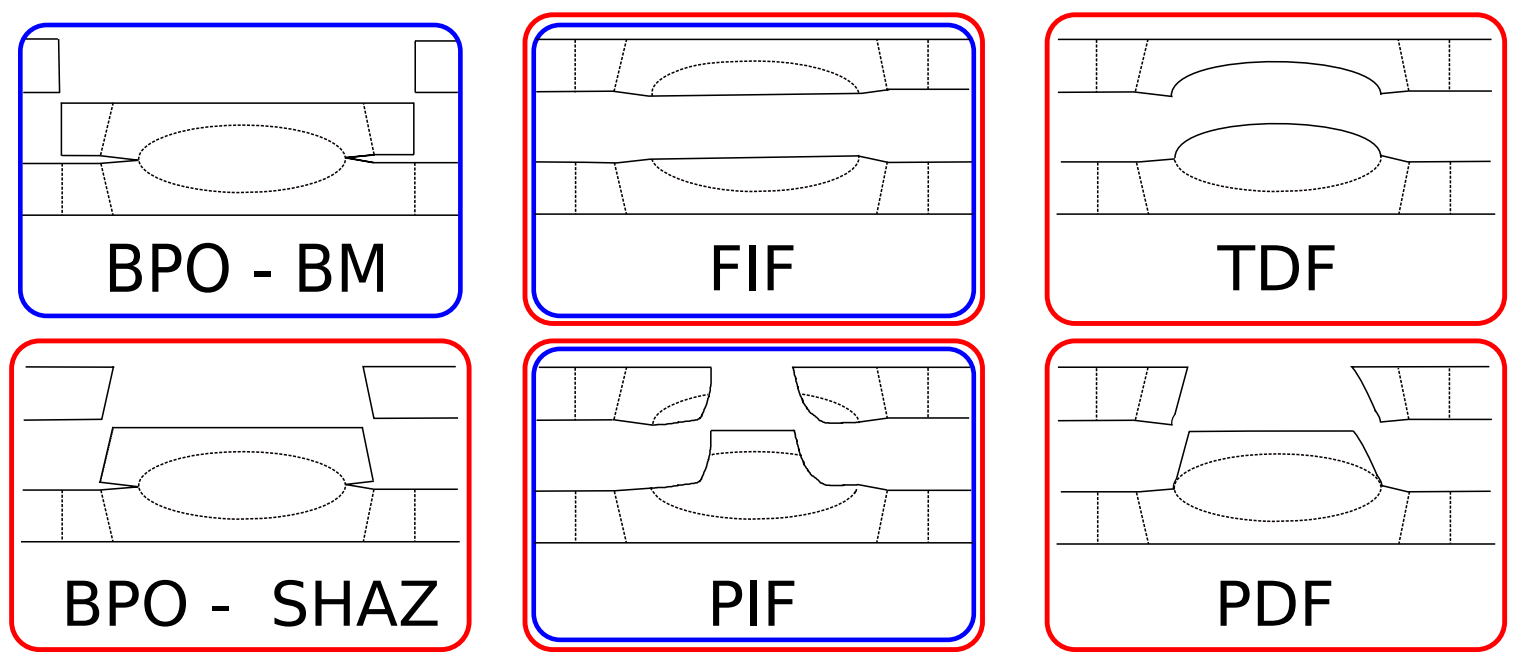

Ductile steels failure modes

AHSS failure modes

Figure 2.9: Scheme of the multiple failure modes represented in cross sectioned spot welds. The colored squares indicate the steel grades where the different failure modes are more typically observed. Notice that in the case of BPO failure occurs at BM in convectional ductile steel grades and at the SHAZ in the AHSS grades.

However, it is more important to analyze in detail the first fail zone than the final appearance to understand the failure behavior and fracture mechanisms. Three main potential fracture zones, and competing failure mechanisms are identified in general in spot welds or different steels ranges as presented in Figure 2.10 [5, 6]: (i) strain localization far away from the nugget (either in BM, or in the SHAZ), (ii) shear between nugget and HAZ, and (iii) semi-brittle fracture through the nugget. They depend on different factors as the base material properties, sheet thickness, spot weld diameter, base material properties, loading case and the combination of all them. In general, a failure mode cannot be neither associated uniquely to a combination of these factors, and a factor configuration cannot be associated uniquely to a failure mechanism. However, it can be concluded that some cases are prone to failure in a failure mechanism. For instance, ductile or mild steels usually fail due to strain 
localization or necking in base material. This type of failure occurs in AHSSs for some loading cases, such as in-plane conditions where the joints fail in SHAZ [18, 39]. However, due to the local properties distribution of AHSS spot welds, these joints fail due to semi-brittle fracture of the nugget due to stress/strain concentration around the notch tip when they are loaded under out-of-plane conditions [6, 10, 17].

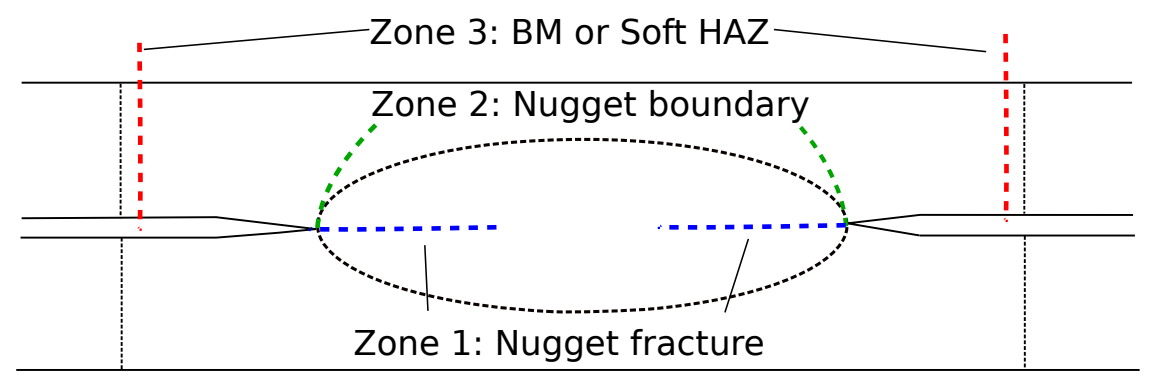

Figure 2.10: Schematic spot weld cross section with different potential failure zones.

\subsection{Spot weld fracture modeling}

The features of spot weld fracture response presented in Section 2.4 should be considered when spot welds are modeled in the FEM full-vehicle crash simulations, introduced in Section 2.1. However, in these full-vehicle models, a good description of strong localized phenomena such as failure of spot welds would need locally fine re-meshing. Due to the Courant-Friedrich-Levy criterion for the critical simulationtime-step, the elements have to be kept of specific minimum sizes to avoid escalating computational costs. Thus, fully resolved detailed models for spot welds are not attainable, tanking into account the great number of spot welds present in the fullvehicle. For this reason, simplified models, where bearing capacity is obtained from basic material properties (e.g. tensile strength), were introduced to describe the spot weld failure. While providing reasonable results for conventional ductile steels, mainly because most failures occur at the sheets rather than at the joints, these models fail to predict the new failure mechanisms of AHSS joints presented in Section 2.3. The maximum force of the AHSS joints was then extracted using data mining and large experimental campaigns. However, due to the thousands of spot welds join sheets of various materials and thicknesses, obtaining the maximum forces for all the possible configurations would be too costly. In order to reduce this experimental test matrix, FEM detailed models have been developed in order to reproduce the new failure 
2.5. Spot weld fracture modeling

modes and predict the maximum forces for multiple configurations. These models use a detailed characterization of the joined material.

\subsubsection{Simplified models}

Over simplistic models were introduced at the beginning of the 2000s to represent the spot weld mechanical response in the full-vehicle crash simulations [7, 40, 41]. These models had different range of complexities, from simple single rigid beam models up to more complex spider configurations or rate dependent models [8, 42]. In most of these models, the spot weld is represented by a simple spring beam with linear elastic properties that joins the shell elements of the mesh of the car structure parts.

Later, fracture was introduced in the over-simplistic beam models. Failure forces are obtained from either analytical formulas considering the base material properties or large experimental campaigns considering multiple steel types [9]. For the analytical formulas characterization methods, $\mathrm{BPO}$ failure is assumed by limiting the force that the beams could bear by considering the weld to be a rigid cylinder and evaluating the plastic collapse of the surrounding sheet upon loading [32, 43]. On the other side, for FIF the maximum forces are calculated using linear elastic fracture mechanics (LEFM), i.e., relating stress intensity factors at the weld notch with the applied forces and comparing the values with the critical material toughness [44, 45]. Furthermore, some post-failure models to represent the evolution of the failed joint after the first crack were introduced [46], as well as models represented mix-modes valid for multiple loading cases [47] .

Other more complex solutions were introduced, such as the special hybrid Trefftz element that represents the spot weld as a rigid cylinder, and solves the stress/strain field in the vicinity of the spot weld nugget with high resolution using analytical solutions. It enables the introduction of more accurate strain-based failure criteria [48.

Some of these models have been industrially implemented in commercial softwares [49], and they have been developed to improve the kinematics constraints modeling between the joined sheets. However, these approaches fail for martensitic boron steels joints because in contrast to other conventional automotive steels, the sheet material in these SWs have comparable mechanical properties to those of weld material [4]. Consequently, weld material can no longer be considered nor rigid neither completely elastic. 


\subsubsection{Detailed models}

The detailed models represent the spot weld using solid finite elements and an elasticplastic material model. Apart from the geometry definition, two features have to be introduced in the model, the local material properties distribution of the spot weld and a suited failure criterion.

In the first models, the mechanical properties heterogeneities around the weld were not introduced [50 52]. The results given by these models showed some qualitative conclusions, such as the stress concentration around the notch tip. However, poor maximum forces estimation could be obtained.

To model the material properties changes, some authors introduced simple rules, where the plasticity hardening curves of the multiple weld zones are scaled using hardness measurements as a scaling factor [13, 53, 56]. A second approach consists on experimentally obtaining the mechanical properties of the weld zones using Gleeble 3500 thermomechanical simulator specimens, where the thermal evolution that is suffered by the HAZ is applied to a base material tensile specimens [10, 16, 29, 39, 57]. A third alternative is to use welded micro specimens, that are designed to develop high strains just in the weld heat-affected zone or the nugget. Two sheets are welded, one of them is separated using a non-thermal process and the other sheet is trimmed in such a way that the HAZ have the most reduced section of the specimen [16, 39, 57, 58]. A particular case of this characterization procedure is the use of miniature tensile bars extracted from the welding zones [59, 60].

Furthermore, in order to define the magnitudes of the geometrical parameters and the dimensions of the HAZ zones, spot weld cross sections and hardness measurements are typically used. Another possibility is to use welding process simulation software (SORPAS $^{\circledR}$, SYSWELD ${ }^{\circledR}$ ), that models the welding process, and the temperature and material changes, given the geometry of the weld and the zones [61 64].

These detailed models introduce different fracture criteria that are detailed in Section 2.5.3. However, all the available SW failure models mentioned above, have not been applied in industry to obtain the maximum forces of the joints, due to the complexity of the material characterization required. 
2.5. Spot weld fracture modeling

\subsubsection{Fracture criteria}

Three types of fracture criteria can be distinguished to model spot weld failure. (i) spot weld models based in the fracture strains of the different zones [56, 58, 64], (ii) spot weld models that introduce a priori the failure zones and give them fracture parameters, i.e. cohesive models [17, 55, 65, 66], and (iii) spot weld models that use fracture mechanics to evaluate the intensity of stress concentration around weld notch [5, 10, 17]. The validity of each one of these fracture criteria depends on the fracture mechanisms observed during spot weld failure as introduced in Section 2.4.

The most used fracture criteria in the full-vehicle models are the strain-based criteria. These ductile fracture criteria are based on fracture strains/stress and have been developed to predict sheet failure. These criteria evaluate the evolution of the strain of the material points using classical FEM simulations and compare it with critical fracture strain values. In these models, the fracture strains are dependent on the loading mode. Some examples are the modified Mohr Coulomb fracture criteria, the Johnson-Cook criterion, the fracture forming limit diagram (FFLD), the Wilkins model or the CrachFEM model [67-71]. Other fracture criteria evaluate the void coalescence and void evolution during material loading and predict fracture using critical void fraction [72, 73]. However, all these previous criteria need a large calibration process and are better suited for sheet fracture than for spot weld fracture.

A second alternative to model spot weld failure are the cohesive zone methods. These methods are used in general in the cases where the fracture surface is known $a$ priori, such as adhesive bonding [66, 74]. However, in the case of spot weld fracture this fracture surface is unknown.

Finally, fracture mechanics methods are the most suited for propagation of cracks in steels [75, 76]. The fracture mechanics theory has been extensively used after the growth of the computational methods. Some theoretical methods based on the fracture variational minimization problem were developed in recent years [77]. However, these models entail a complete new implementation in the commercial software, which is beyond the scope of this thesis. Rice introduced a quantity to predict the crack growth in lineal elastic materials using the energy release rate, the $J$-integral [78]. The concept of the $J$-integral was extended to three dimensional, and non-lineal crack configurations considering also the plastic strains [2, 79].

In previous works [5, 6, 10, 17, 60, 80, the approach of $J$-integral evaluation around weld notch is used to predict the fracture response of AHSSs spot welded 
joints. The stress concentration around the notch is evaluated using $J$-integral and compared to a critical value. However, the critical value of $J$-integral is related with the nugget fracture toughness and the characterization of this mechancial material property is not an easy task. The specimens that are usually employed for this purpose, such as the fracture mechanics compact specimen, the disk-shaped compact specimen, or the single-edge-notched bend SENB specimen [44], cannot be extracted from a nugget due to its minute dimensions (around $6 \mathrm{~mm}$ diameter and $2 \mathrm{~mm}$ high).

Several approaches have been developed to extract these critical values of the spot weld nugget material for different steel grades [19, 60, 80, 82]. The values obtained in these works must be taken with caution due to the uncertainties in the experimental set ups, the variability of the results and some assumptions that are taken to evaluate the $J$-integral using analytical equations. Two important qualitative conclusions can be obtained from previous works where the material fracture toughness of steels are analyzed. The critical $J$-integral varies depending on the fracture mode described in Figure 2.11, i.e. mode I or opening mode has different critical fracture toughness $\left(J_{I C}\right)$ than mode II or sliding mode $\left[J_{I C}\right][81,83]$. Additionally, some authors propose that due to the fact that nugget material is martensitic for most of the steels, the nugget fracture toughness is always the same, independently of the base material steel properties [19]. However, previous literature show also a strong variation on the fracture toughness of martensitics steels with hardness values close to the ones measured in the spot weld nuggets [84].

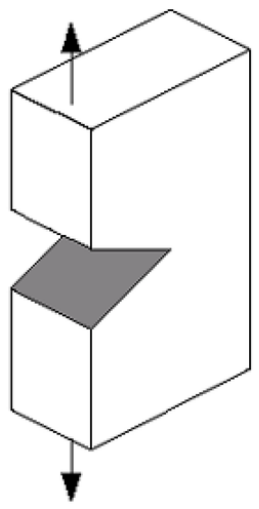

Mode I Tensile

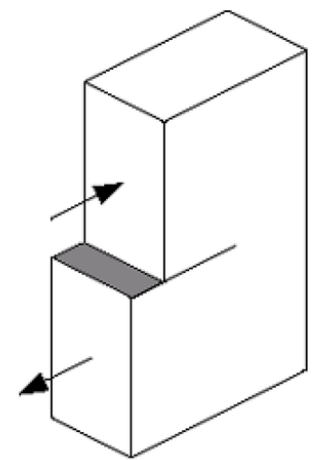

Mode II In-plane

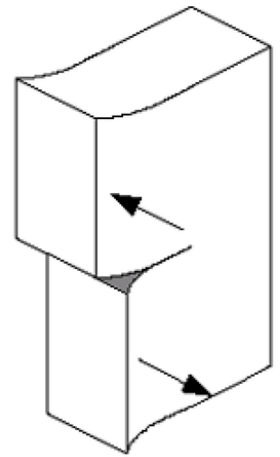

Mode III Anti-plane

Figure 2.11: Fracture modes. 


\section{Chapter 3}

\section{Resistance Spot Weld model}

A detailed FEM spot weld model to simulate the fracture response of this type of joints is developed in this chapter. The model must be simple enough for industrially attainable computational costs while having enough accuracy to be able to capture the different fracture modes. In the next chapters of this thesis an experimental validation campaign of the presented spot weld model is carried out using a specifically designed joining specimen that is loaded up to fracture. This specimen consists of two U-profiles joined using the RSW technology that are loaded at different angles.

The governing equations of the spot weld FEM model are presented in Section 3.1 in order to better understanding the related numerical issues. The applied constitutive model is then introduced in Section 3.2 and it is completed in Section 3.3 with the local material properties definition related with the welding process. The geometry and the mesh of the spot weld and the specimen used in the experiments is presented in Section 3.4. Furthermore, Section 3.5 presents the boundary conditions used in the FEM simulations used to reproduce the experimental set up of the loading tests. In Section 3.6, an energetic fracture criterion based on the use of the $J$-integral is identified as the better suited for the purposes of this thesis. Thus, a computational evaluation of the $J$-integral from FEM simulation data is proposed, with special attention to important features as the identification of the critical notch section, the first propagation direction, and mesh dependency. The numerical issues related with the commercial software used in this thesis are described in Section 3.7 and some illustrative preliminary results are presented in Section 3.8. 


\subsection{Governing equations}

Due to the industrial purposes of this thesis, the finite element model is solved using the commercial explicit Virtual Performance Solution (VPS) software, also known as Pamcrash [85]. This code is able to solve explicit standard finite element problems. Furthermore, some special user-libraries specially implemented in VPS for spot weld material distribution properties have been used. These user-liberies are included in the modular material model (MMM) framework [86]. Furthermore, a code has been developed for the evaluation of the $J$-integral using the data obtained from the standard FEM simulations performed with VPS and MMM.

VPS Explicit is a dynamic code, and consequently is based in the momentum equation defined by

$$
\rho_{0} \frac{\mathrm{D} \boldsymbol{v}}{\mathrm{D} t}-\nabla \cdot \boldsymbol{\sigma}=\rho_{0} \boldsymbol{b}
$$

where $\rho_{0}$ indicates the density of the material, $\frac{\mathrm{D}}{\mathrm{D} t}$ indicates the material time derivative, $\boldsymbol{v}$ is the velocity, $\boldsymbol{\sigma}$ is the Cauchy stress tensor and $\boldsymbol{b}$ are the internal forces. The right-hand term of this equation, i.e., the internal forces, are zero. Due to the low velocities applied during the experimental loading tests, the first term of this equation can be neglected. However, this term cannot be disabled in the VPS simulations, i.e., quasi-static simulations cannot be performed. Due to the necessary small size meshes for strain field concentration solving (see Section 3.6.4) as well as the necessary small time steps (product of the Courant-Friedrichs-Lewy condition [87]), large computational times are needed. More details about the computational time optimization used to perform the simulations of this work are given in Appendix B.

\subsection{Constitutive model}

VPS is implemented at finite strains [85, and thus the constitutive relations are given by

$$
\boldsymbol{\sigma}^{\nabla J}=\mathbb{C}_{e l}^{\sigma J}: \boldsymbol{D}^{e}
$$

where $\boldsymbol{\sigma}^{\nabla J}$ is an objective rate of the Cauchy stress and $\mathbb{C}_{e l}^{\sigma J}$ is the elastic moduli. An objective rate of the Cauchy stress, i.e. the Jaumann rate of the Cauchy stress, 
is used in order to fulfill the objectivity integration criteria [88]. The Jaumann rate of the Cauchy stress is defined by

$$
\boldsymbol{\sigma}^{\nabla J}=\frac{\mathrm{D} \boldsymbol{\sigma}}{\mathrm{D} t}-\boldsymbol{W} \cdot \boldsymbol{\sigma}-\boldsymbol{\sigma} \cdot \boldsymbol{W}^{T}
$$

where $\boldsymbol{\sigma}^{\nabla J}$ is the stress tensor and $\boldsymbol{W}$ is the spin tensor defined as $\boldsymbol{W}=\frac{1}{2}\left((\nabla \boldsymbol{v})^{T}-\right.$ $\nabla \boldsymbol{v})$. Notice that no damage is introduced in the model.

Continuing with the terms of Eq. $3.2, \boldsymbol{D}^{e}$ is the elastic rate-of-deformation defined as $\boldsymbol{D}^{e}=\boldsymbol{D}-\boldsymbol{D}^{p}$, where $\boldsymbol{D}$ is the total rate-of-deformation which is given by $\boldsymbol{D}=$ $\frac{1}{2}\left(\nabla \boldsymbol{v}+(\nabla \boldsymbol{v})^{T}\right)$, and $\boldsymbol{D}^{p}$ is the plastic rate-of-deformation given by a plastic flow rule.

Furthermore, $J_{2}$ plasticity, that is based on a von Mises yield surface, is the flow theory followed in this work. Therefore the yield condition is given by

$$
f(\boldsymbol{\sigma})=\sigma_{e q}-\sigma_{k}
$$

where $\sigma_{k}$ is the yield stress of the material that is related with the accumulated plastic strain using the hardening curves, and $\sigma_{e q}$ is the equivalent von Mises stress defined as $\sigma_{e q}=\sqrt{\frac{3}{2} \boldsymbol{\sigma}^{D}: \boldsymbol{\sigma}^{D}}$, where $\boldsymbol{\sigma}^{D}$ is the deviatoric stress tensor defined as $\boldsymbol{\sigma}^{\boldsymbol{D}}=\boldsymbol{\sigma}-\frac{1}{3} \operatorname{tr} \boldsymbol{\sigma} \boldsymbol{I}$. This model suggests that material yielding begins when the equivalent von Mises stress reaches a critical value.

Due to the welding process, the local yield stress and the hardening curves are heterogeneous in the weld vicinity as described in Section 2.3. This heterogeneity is correlated with the $\mathrm{HV}$ distribution measured experimentally in the cross section of the welds. This modeling of the multiple material zones is presented in the Section 3.3 . Finally, the description of fracture modeling is presented in Section 3.6.

\subsection{Local material properties}

In order to properly account for the material properties distribution of the spot weld in AHSS joints, the mesh is divided in multiple zones that are modeled with the corresponding material properties. Three welded zones are modeled as presented in Figure 3.1: (i) the FZ or nugget, (ii) the HAZ that can be subdivided in two zones called SHAZ and HHAZ, (iii) and the BM.

The nugget is represented as an ellipse rotated around an axis normal to the sheet plane. The mayor axes of this ellipse are given by the spot weld diameter (see 
Figure 2.6), and the minor axis is one third of the sum of the both sheets' thickness. Therefore, the resulting function to define the oblate spheroid geometry of the fusion zones is

$$
\frac{x^{2}}{r_{n}^{2}}+\frac{y^{2}}{r_{n}^{2}}+\frac{z^{2}}{t_{n}^{2}}=1
$$

where $r_{n}$ is the nugget radius, and $t_{n}$ is defined as $t_{n}=\frac{t_{1}+t_{2}}{3}$ where $t_{i}$ are the thicknesses of both sheets (see Figure 3.1). The heat affected zones are those regions confined in between the fusion zone border and a surface depicted also by ellipses. The sizes of all ellipses depend mainly on the size of the spot weld, i.e. on the weld diameter.

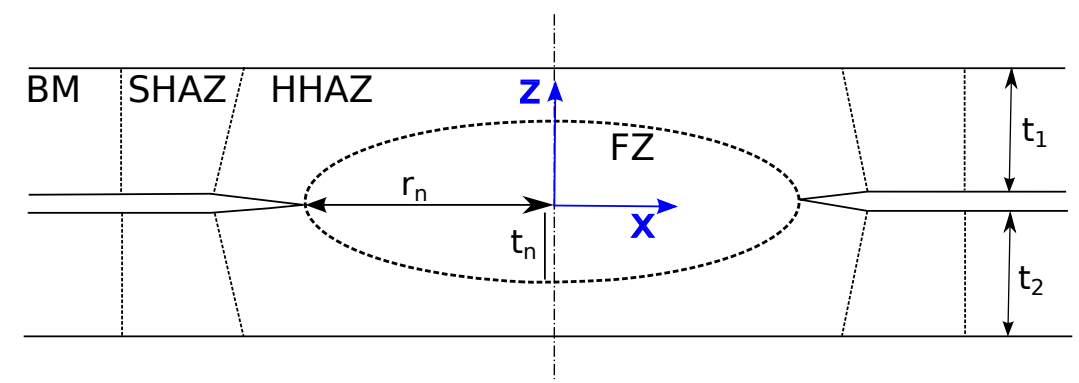

Figure 3.1: Scheme of cross sectioned spot weld with the modeling zones.

The characterization of material properties of the martensitic boron steel spot welds made by Eller et al. [18, 64, 89] are used in the simulations of the current chapter. In these works, the hardening curve at each point is correlated with the HV of the 22MnB5 material. The $\mathrm{HV}$ values at each point are extracted experimentaly from cross-sectioned spot welds and are introduced in the FEM model.

\subsection{Geometry and mesh description}

The geometry of the joining specimens is defined using a orphan mesh as depicted in Figure 3.2. Only one quarter of the sample is modeled to reduce computational costs. The generated mesh has 3D uniformly distributed under-integrated hexahedral 8 -node solid elements with one integration point. The element size is around $1.5 \mathrm{~mm}$ in the sheets and is reduced to $0.1 \mathrm{~mm}$ in the vicinity of the nugget. The resulting mesh has approximately five hundred thousand elements. 
3.4. Geometry and mesh description

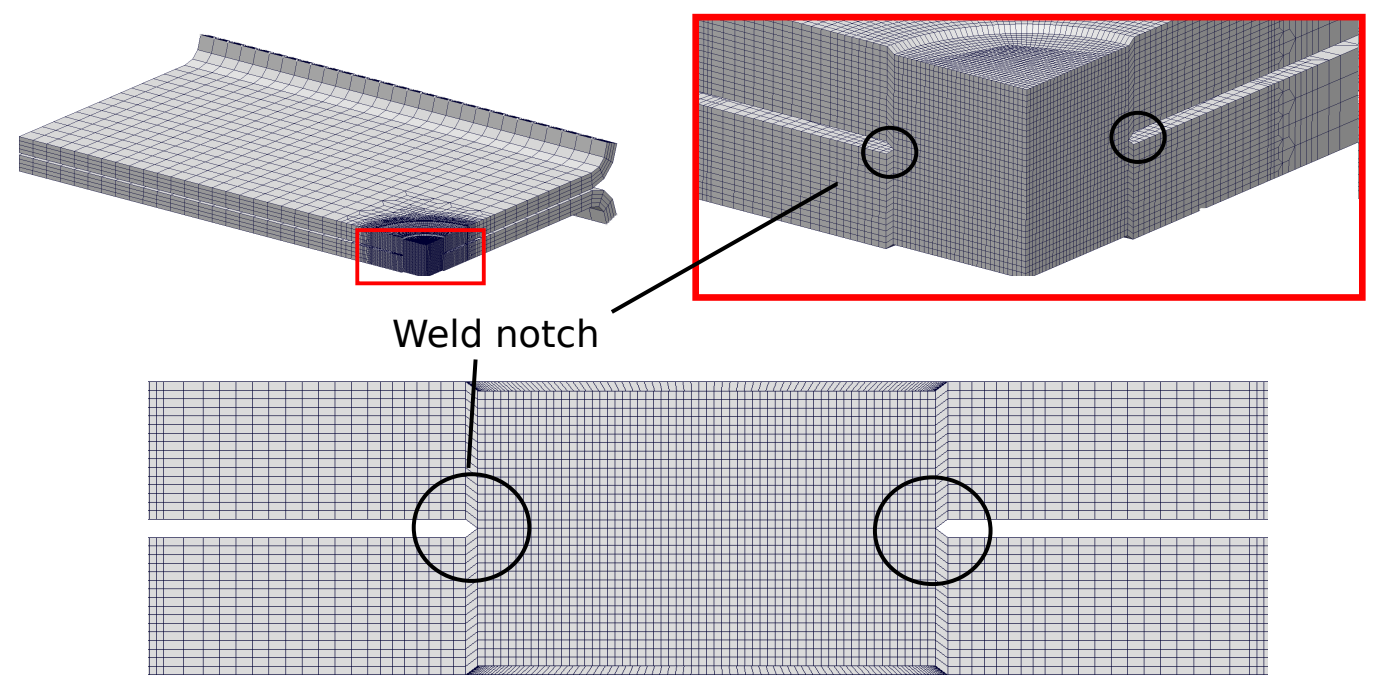

Figure 3.2: Mesh of the loading specimen and the spot weld model. From top left to bottom right: Quarter model of the specimen, zoom in the spot weld zone, and detail of the mesh in a front view of the cross sectioned specimen.

The welding process generates a particular spot weld geometry that is shown using the finite element mesh in Figure 3.2. The main geometrical features described in Figure 2.6 are introduced. Two sheets are joined by a circle joining surface with a corresponding joining diameter. The dimension of this diameter is extracted from experimental observations. The weld notch, with a strong influence in the fracture response as presented in Section 3.6.1, is modeled by using an acute angle. Finally, the electrode indentation is represented in both sides of the sheets. A detailed analysis of the most suited mesh size is given in Section 3.6.4.

It is important to note that, the actual weld notch shape is extremely irregular and depends on several factors, such as the applied electrode forces, the sheets protection coating and the welding expulsions. This irregularity may influence on the strain field around the weld during loading and consequently on the bearing capacity. In order to quantitatively asses this influence, two different notch shapes are calculated in previous simulations as can be seen in Figure 3.3, a square weld notch and a sharp notch. Slight differences are observed between the strain field around the notch for both shapes. However, due to the extreme localization of the strains, and the high strain gradients, some very localized elements have completely different strain values depending on the notch shape. This effect causes these elements to fail earlier 
leading to different calculated fracture forces if a strain-based fracture criterion is used. However, when energy release rate approaches, such as a $J$-integral based fracture criterion, are applied, the effect of localized strains is regularized and the influence of the notch geometry is minimized.

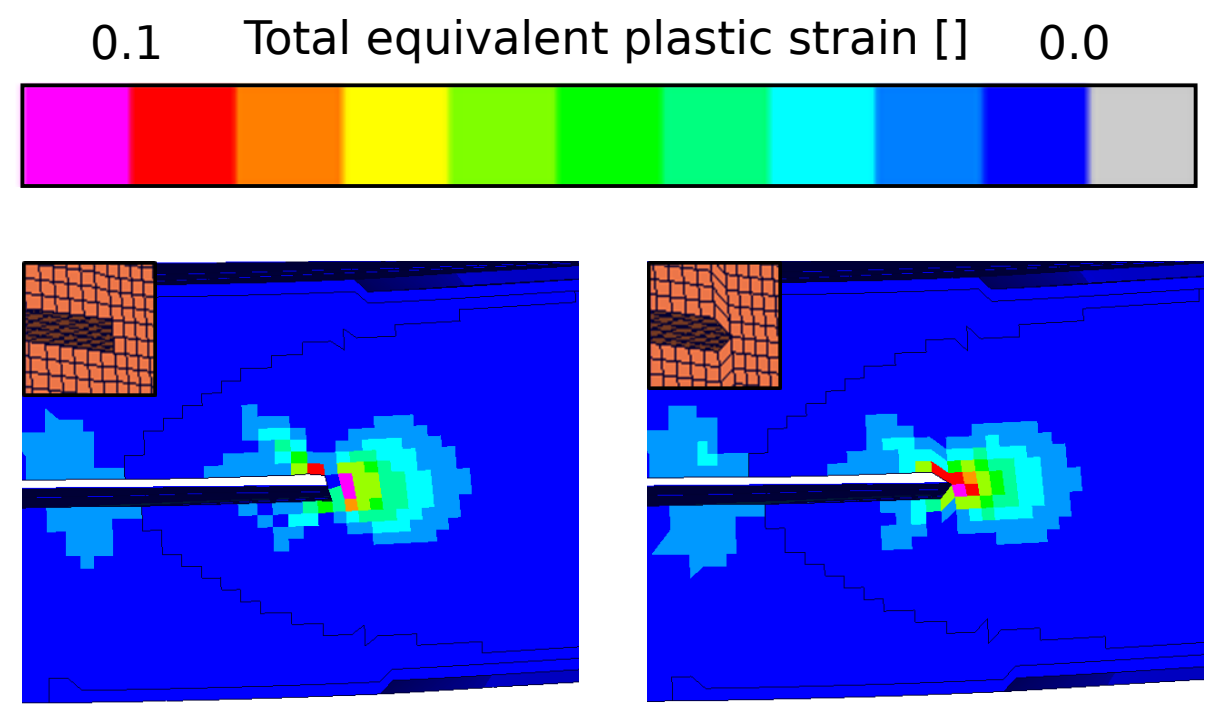

Figure 3.3: Strain field around weld notch for two different shapes (square on the left, sharp on the right).

\subsection{Boundary conditions}

The boundary conditions represent the experimental set up of the loading tests that will be presented in the following chapters of this work, in which the two welded U-profiles are loaded up to fracture at three different angles using a Zwick machine. The bottom sheet is fixed using zero displacement and zero rotation constraint in a set of nodes at the end of the U-profile flanges (see Figure 3.4). It represents the zone where the specimens are screwed to the clamping device. In the other sheet, a vertical displacement boundary condition is applied to a set of nodes of the corresponding flanges and the displacement in the other directions and all rotations are locked. The specimens are rotated to represent the correct loading angle as shown in Figure 3.4.

Due to the low loading velocities, the problem could be treated as quasi-static, i.e. the time of the simulations has no physical meaning. The used code - VPS- does not allow to perform this type of simulations. In order to reduce computational times and 
3.6. Spot weld fracture modeling in AHSS

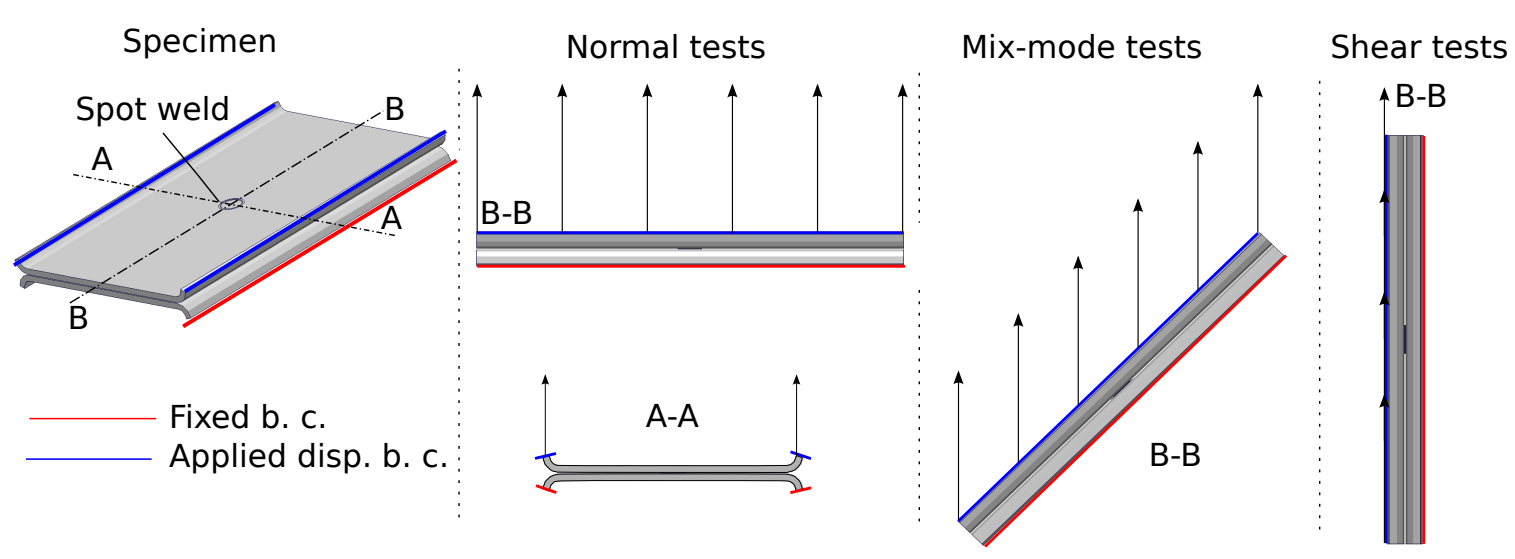

Figure 3.4: Scheme of loading angles and boundary conditions. Arrows indicate direction of applied displacement.

avoid spurious waves, a constant acceleration is applied instead of displacement ramp as boundary condition. More detailed explanations can be found in Appendix B.

\subsection{Spot weld fracture modeling in AHSS}

In the case of AHSS spot welded joints, BPO fracture mode due to necking instability at the SHAZ under spot weld in-plane loading conditions (see Figure 2.9 and Figure 2.10 has been extensively studied in previous works [6, 10, 15, 64]. This situation is typically observed when a large welded vehicle structural component is bended or loaded from two opposite zones, and the welded zone enters in necking regime leading to catastrofic failure.

However, when during a crash situation two fifferent welded structural components are trying to be separeted from each other, i.e. when the spot welds are loaded under out-of-plane conditions (see Figure 3.5), nugget rupture has been observed initiating at the weld nocth (see Figure 2.10). This situation may lead to complete separation of both connected sheets resulting in structure collapse. A suited fracture criterion for this type of failure in AHSS spot welded joints is still missing. 

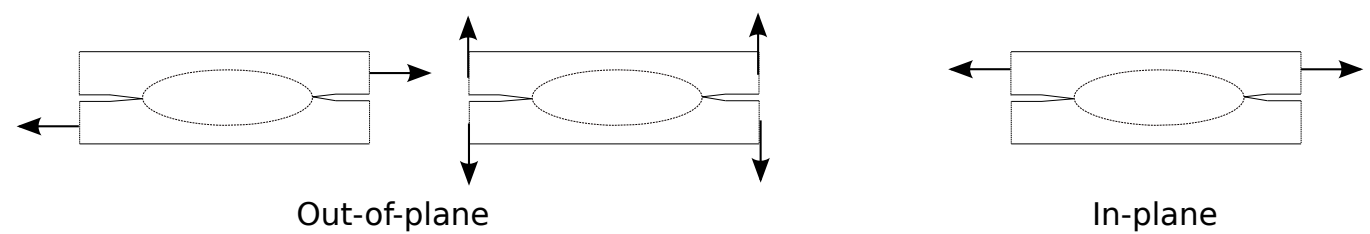

Figure 3.5: Different spot weld loading modes: In-plane and out-of-plane loading conditions.

\subsubsection{Spot weld failure in AHSS under out-of-plane loading conditions}

Ductile steels have low yield strengths and large fracture strains, whereas high yield strengths and small fracture strains are typical in the case of AHSS (see Figure 2.3). To illustrate the different critical failure zones in the case of spot weld loading depending on these welded material features, a shear test of joints of two different steel grades have been modeled and simulated. On the one hand a spot weld with a high strength $\mathrm{BM}$ and a soft $\mathrm{HAZ}$ is modeled in order to represent a AHSS joint. On the other hand the response of a spot weld with both the BM and the HAZ modeled with low yield strength material is calculated representing a ductile steel. In both cases the FZ is modeled as a high strength material. In order to have a common criterion to assess the proximity to the fracture for both types of hardening curves, an accumulated damage parameter $(D)$ is used:

$$
D=\int_{0}^{\bar{\varepsilon}_{p}} \frac{d \bar{\varepsilon}_{p}}{\varepsilon_{f}(H V)}
$$

where the effective plastic strains $\left(\overline{\bar{\varepsilon}_{p}}\right)$ are compared with the plastic strain to fracture $\varepsilon_{f}$ which depends on the hardness $(H V)$ and the strain hardening curve of the corresponding geometrical zone [90]. Notice that in this case this is just a qualitative criterion to find the critical failure zones.

In Figure 3.6 it can be seen that the main critical zone is situated at the BM in the case of ductile materials in which failure is induced by sheet necking in the experimental observations. However, for AHSS the BMs have similar properties to those of the fusion zone. Therefore, in agreement with experimental observations [6, 10, 11, 17, the critical zone is shifted to the weld notch due to the stress concentration leading to failure in zones 2 and 3 following the scheme of Figure 2.10.

A fracture criterion that assesses the severity of stress/strain concentration ahead 


\section{Accumulated damage [-]}

\section{1}

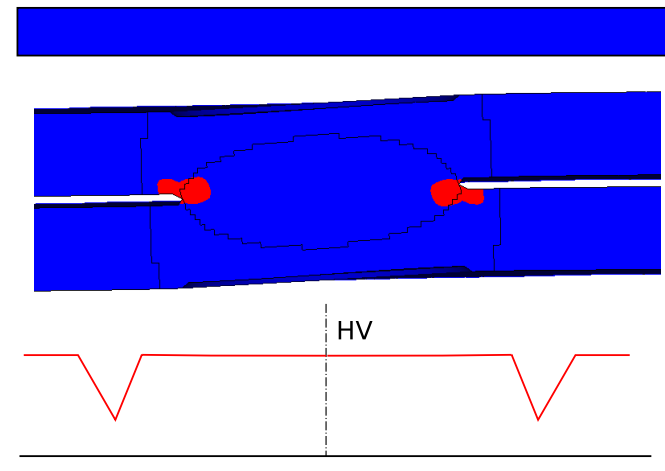

Hard BM with soft HAZ
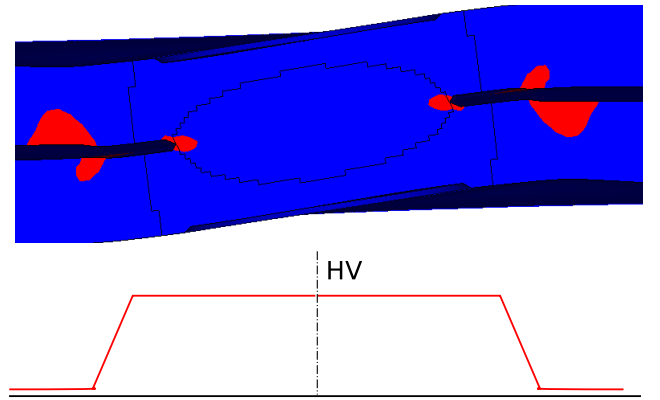

Soft BM

Figure 3.6: Critical zones of spot weld in a shear loading case for a High Strength Steel (left) and a ductile steel (right).

of the weld notch is necessary. Fracture models such as eigendeformation approaches [77, 91, 92] that predict new crack features (e.g. crack propagation) is undesirable due to the high computational costs. The fracture criterion must therefore be simple and robust in order to be able to reproduce all loading and geometrical alternatives without large experimental calibration. The Rice $J$-integral-based criterion is the ideal choice beacause of the compromise between simplicity, low computational costs and correct prediction of the spot weld fracture trigger. Thus, it is used in this thesis for the assessment of the stress field intensity around the weld notch [78]. The $J$-integral was defined for a two dimensional linear elastic cracked body as:

$$
J_{k}=\int_{\Gamma}\left[W n_{k}-\sigma_{i j} \frac{\partial u_{i}}{\partial x_{k}} n_{j}\right] d s
$$

where $\Gamma$ is a path beginning at the bottom crack face and ending on the top face (see Fig $3.9(\mathrm{a}), \boldsymbol{n}$ is the outward normal to $\Gamma, \boldsymbol{\sigma}$ the stress tensor, $\boldsymbol{u}$ the displacements, $d s$ the increment of arc length along $\Gamma$ and $W$ the total strain energy density defined by $W=\int_{0}^{\varepsilon} \sigma_{i j} d \varepsilon_{i j}$, where $\varepsilon_{i j}$ are the components of total strain tensor $\varepsilon$. The subindex $k$ defines the coordinates of the crack tip coordinate system (see Figure 3.9(a).

The $J$-integral is equivalent to the strain energy release rate that is a measure of the energy available for an increment of crack extension. When it reaches a critical value, which is a material property related with fracture toughness, a crack nucleates and the joint fails. The extension of this concept to elastic-plastic materials is presented next. 


\subsubsection{Evaluation of the $J$-integral in elastic-plastic materials in FEM frame work}

The Equation 3.7 is defined for a two dimensional crack and uses strains and stresses in a crack surrounding path $\Gamma$. It was introduced for linear elastic materials to be evaluated with the assumption of LEFM.

The AHSSs have a brittle response, and could be considered a priori elastic up to fracture under the modeling point of view. Pure normal and shear test simulations using pure elastic and elastic-plastic material have been performed to evaluate this assumption. The obtained stress field of both cases are presented in Figure 3.7. Higher stresses are observed for the elastic case due to the singularity created by the notch that is limited by the yield strength in case of elastic-plastic material. It must be noticed, that the zone where stresses higher than $1.0 \mathrm{GPa}$ (i.e. the raw material yield strength) are reached is very confined in both modeling approaches.

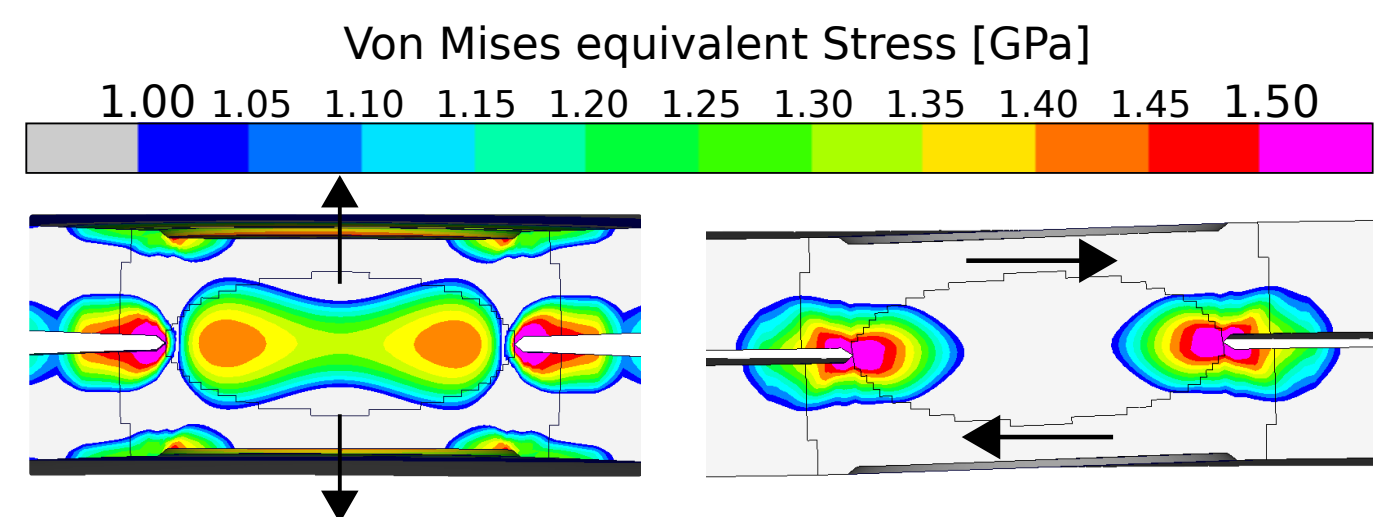

(a) Elastic-plastic material.
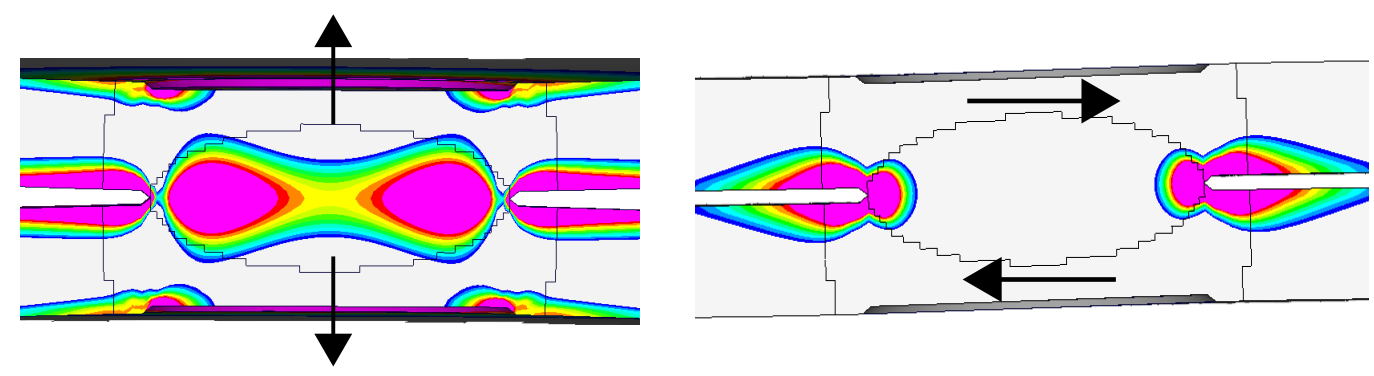

(b) Pure linear elastic material.

Figure 3.7: Stress distribution around weld notch. Left: Normal test. Right: Shear test. 
However, despite the fact that the plastic zone is extremely confined around the notch, the forces-displacement curves that are compared in Figure 3.8 show that the results of pure elastic modeling are not valid for neither normal tests nor shear tests. For this reason, the LEFM hypothesis cannot be used here.

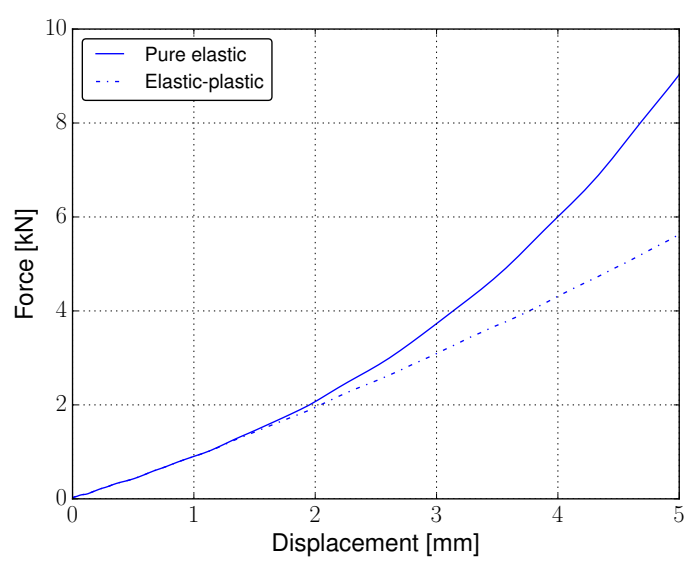

(a) Normal test.

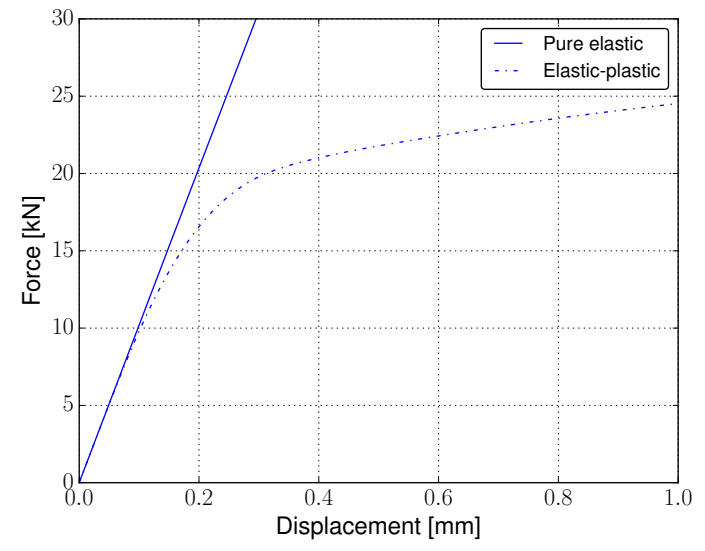

(b) Shear test.

Figure 3.8: Force displacement curves comparing pure lineal elastic and elastic plastic models.

Furthermore, the weld notch, which has the role of crack front in the spot welds, has a three dimensional geometry, defined as a circle around the weld nugget. Therefore, the $J$-integral must be computed point-wise at each position of this crack front. The critical section at which the $J$-integral must be computed along this crack front or weld notch is analyzed in Section 3.6.3.

In this thesis, the computation of the $J$-integral has been performed using an equivalent domain integral [2, 79, 93 95], that evaluates the $J$-integral in a 3D domain around the notch tip gathering the results from the standard FEM simulations following the constitutive model presented in Section 3.2 and Section 3.3 . The computation of the $J$-integral using a domain (volume) integral is numerically advantageous since the capture of the singular field details near the crack front becomes unnecessary giving more accurate results than the equivalent contour integral. The details of the evaluation of the $J$-integral in FEM are presented in Appendix A. In this framework, the $J$-integral yields

$$
J_{1}=\frac{1}{f}\left[\int_{V}\left(\sigma_{i j} \frac{\partial u_{i}}{\partial x_{1}} \frac{\partial s}{\partial x_{j}}-W \frac{\partial s}{\partial x_{1}}\right) d V\right]
$$


where $V$ is the volume of integration domain that is a hollow cylinder that surrounds the a small portion of the notch at the correct weld section. This cylinder is defined by radii $r_{\min }$ and $r_{\max }$ (see Figure 3.9(b) and a height. This height must be small but enough to contain at lest four elements in the cylinder axial direction. $s$ is an arbitrary defined function that enables the generation of an area/volume integral and the value of $f$ is obtained from the $s$-function value. The fields are expressed in the local coordinate system of axes $x_{1}, x_{2}, x_{3}$, shown in Figure 3.9(b).

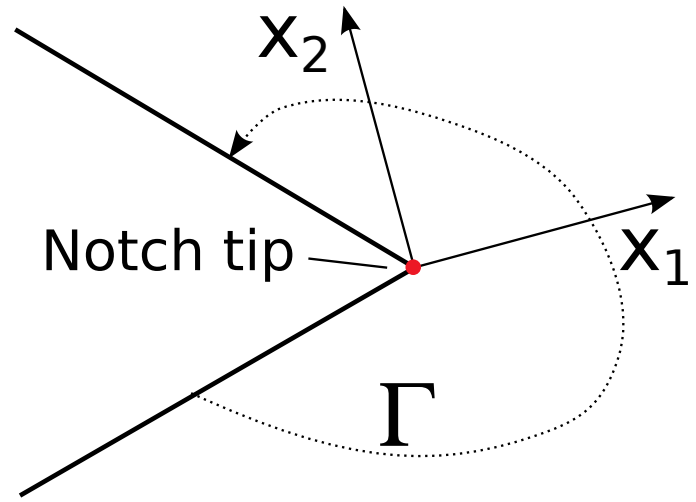

(a) Two dimensions.

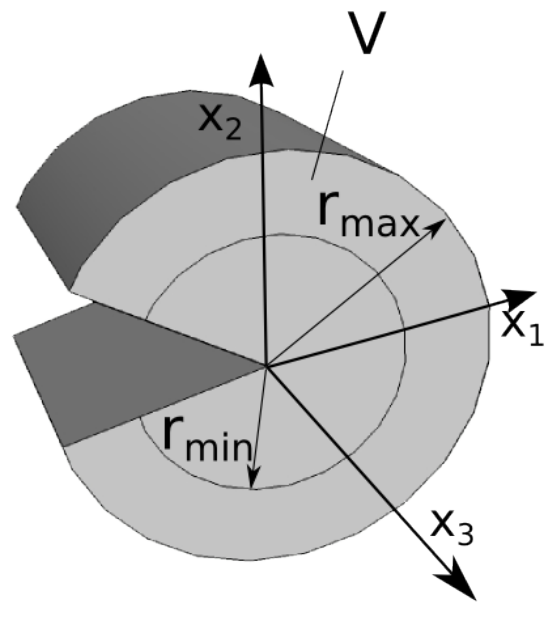

(b) There dimensions.

Figure 3.9: Crack tip coordinates and the domain where $J$-integral is evaluated.

It must be noticed, that the procedure presented in Appendix A follows the conventional $J$-integral definition, i.e. the material is considered non-linear elastic, and consequenly, there are not plastic strains. However, this assumption is obviously not valid for elastic-plastic materials where the total strain has contributions from both elasticity and plasticity. Recently, the concept of $J$-integral has been derived to elasto-plastic materials with the so-called $\overline{J^{e p}}$ in accordance with incremental theory of plasticity [96, 97]. The definition of this $J^{e p}$ considers that $W$ in Eq. 3.7 should include only the elastic strains, since plastic strains do not contribute to the fracture energy. In order to achieve path-independent results in the computation of $J^{e p}$, the selected path, defined by the compuntational volume, must be completely enclosed in the elastic zone where not plastic strains are present. Following Figure 3.10, it can be seen when $r_{\min }$ and $r_{\max }$ are defined as $1.3 \mathrm{~mm}$ and $1.5 \mathrm{~mm}$ respectively, 
the computational domain doesn't contain material with plastic strains, i.e. material with von Mises equivanlent stress higher than material yield stress (1 GPa) and, consequently, the conventional $J$-integral procedure is equivalent to the $J^{e p}$ and it is therefore correct.

Figure 3.10 presents also the results of the conventional $J$-integral using multiple values of $r_{\min }$ and $r_{\max }$. The size domain independency can be observed for almost all cases, with the only exception of an extremely small domaing in the vicinity of the notch, already reported in previous works [97].

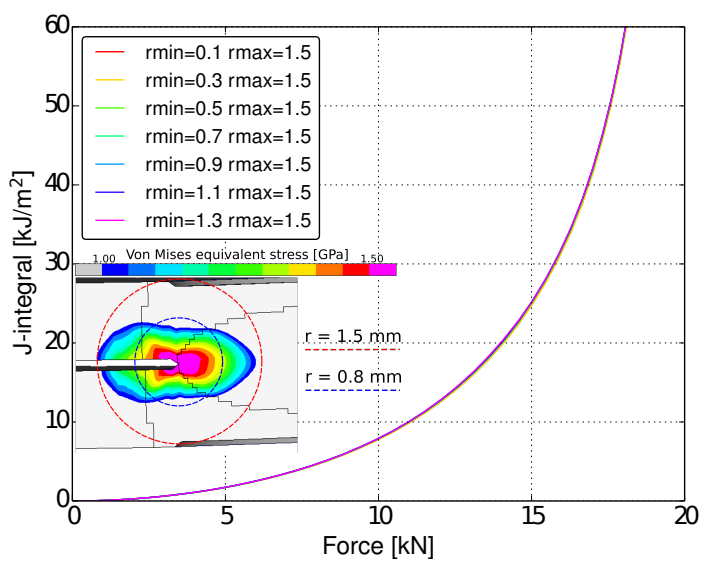

(a) $r_{\min }$ independency.

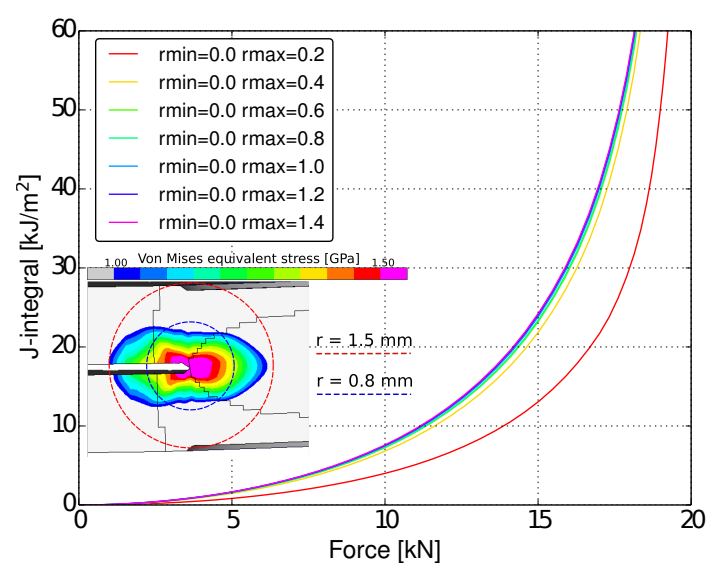

(b) $r_{\max }$ independency.

Figure 3.10: $J$-integral evaluation using multiple computational domain sizes. Almost all curves are overlapped, only an exception can be noticed in the case of $r_{\min }=$ $0.0 \mathrm{~mm}$ and $r_{\max }=0.2 \mathrm{~mm}$.

In summary, in order to quantify the stress/strain concentration in the weld notch and predict the failure of joints due to crack propagation from the weld notch, the next procedure is followed:

1. Calculate the resistance spot weld model response of the loading tests using standard FEM. The numerical features introduced in the previous sections are used for this step.

2. Compute the point-wise $J$-integral gathering the data obtained in the previous step and using the equivalent domain integral. The $J$-integral must be computed in the critical crack advance direction and at the critical weld section following the process detailed in Section 3.6.3. 
3. Compare the $J$-integral values with a critical material value, which is related with the fracture toughness of the nugget material. The applied force at which this value is reached corresponds with the joint bearing capacity.

\subsubsection{First propagation direction and weld cross section}

The first crack advance direction must be selected before evaluating the $J$-integral to set $x_{1}$ (see Figure 3.9(b) . Two approaches are used in this thesis to obtain it, observations of the failed experimental test and the use of a numerical method. The numerical method is based on a closed-form expression that determines the material onset of instability [98]. This expression is obtained from the instability condition given by

$$
(\boldsymbol{g} \otimes \boldsymbol{h}): \mathbb{C}_{e p}:(\boldsymbol{g} \otimes \boldsymbol{h}) \geqslant 0, \forall \boldsymbol{g}, \boldsymbol{h}
$$

where $\mathbb{C}_{e p}$ is the tangent elastic-plastic matrix. The vector $\boldsymbol{h}$ that maximizes the left-hand side of the equation determines the crack advance direction. The spot weld model proposed in this thesis doesn't include damage, therefore damage related terms in [98, are set to zero.

Moreover, the $J$-integral must be evaluated at the position the weld notch, at which the crack advance takes place first. This critical section of the crack front depends on the loading conditions and is analyzed in Section 3.8.1.

\subsubsection{Mesh size and convergence}

In the industry contest, uniform mesh are typically used. However, in the case of notched geometries mesh refinement is necessary in the vicinity of notch tip when using standard FEM in order to capture the notch tip stress/strain gradients. The spot weld model presented in this chapter has been simulated with different mesh sizes, from an element size of $0.4 \mathrm{~mm}$ to an element size of $0.05 \mathrm{~mm}$. It can be seen in Figure 3.11 that even with a mesh of $0.05 \mathrm{~mm}$ the strain field in the vicinity of the notch tip is still not fully resolved. However, the use of uniform meshes and an explicit dynamic FEM software (see more details in Appendix B leads to extremely high computational costs when a mesh size lower than $0.05 \mathrm{~mm}$ is used. For this reason, the force-displacement curves for different mesh sizes are presented in Figure 3.12(a) to check the influence of the mesh size in the obtained results. Slight differences are 
observed between $0.01 \mathrm{~mm}$ and $0.005 \mathrm{~mm}$ element size, for this reason $0.1 \mathrm{~mm}$ has been selected as the suited mesh size for the purposes of this thesis.

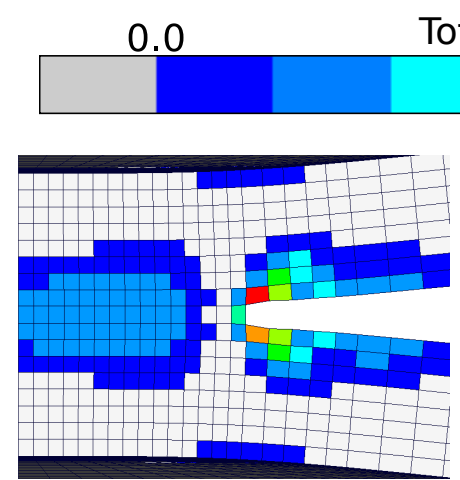

M0.2
Total equivalent plastic strain

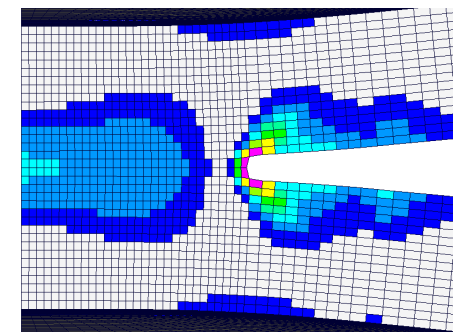

M0.1
0.1

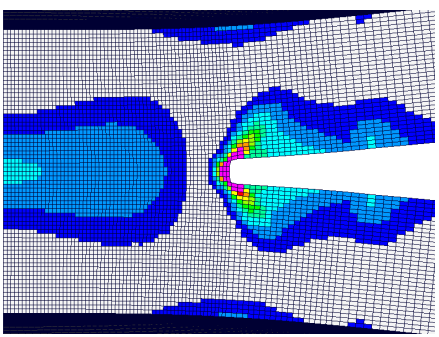

M0.05

Figure 3.11: Strain fields around weld notch using different mesh sizes. M0.2, M0.1, M0.05 indicate $0.2,0.1$ and $0.05 \mathrm{~mm}$ average mesh sizes respectively.

This strong mesh size dependency when solving strain fields leads to strong mesh dependency of the strain-based fracture criteria. This mesh dependency is reduced when the $J$-integral is used as fracture criterion. The dependency of the $J$-integral when using different mesh sizes is shown in Figure 3.12(b). The $0.1 \mathrm{~mm}$ mesh size is considered valid for the purposes of this thesis due to the sligh differences when comparing with $0.05 \mathrm{~mm}$ results.

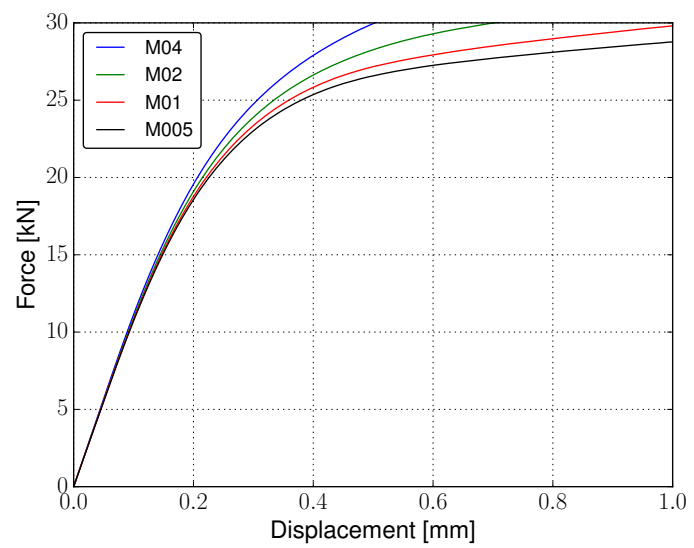

(a) Force displacement curves.

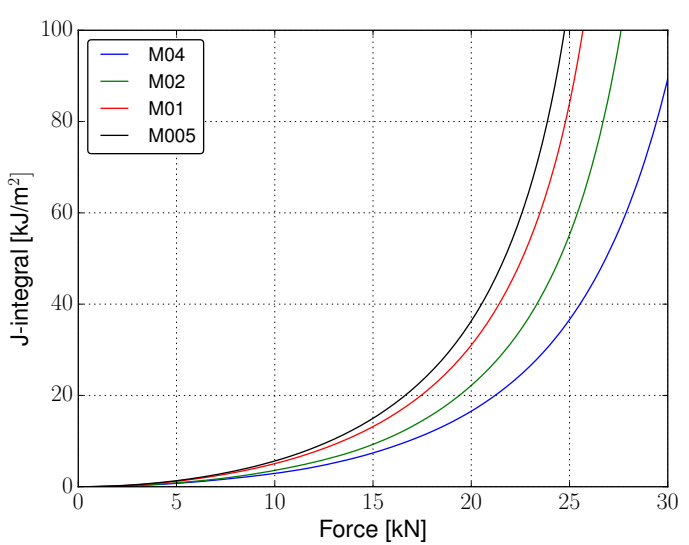

(b) J-integral evaluation.

Figure 3.12: Previous simulation loaded spot weld shear tests results at multiple meshes. M04, M02, M01, M005 indicate 0.4, 0.2, 0.1 and $0.05 \mathrm{~mm}$ average mesh sizes respectively. 


\subsection{Numerical issues}

The commercial code used in this thesis, i.e. VPS, has been specially developed for full-vehicle simulation models. It has some non-customizable modeling features suitable for these types of large scale simulations, such as explicit dynamics and large strains, that are cuestionable for the characteriztion of the spot weld failure. In order to check the validity of the simulations presented in the current thesis, a comparison of the numerical results between the results obtained with VPS and with a more customizable commercial software (i.e. ABAQUS/Standard) is given in this section.

VPS is an explicit dynamic code. However, because of the low applied velocities during the experimental tests a static code would be a priori suitable and would reduce the computational costs. Normal tests are simulated using the commercial implicit static code ABAQUS/Standard to check the influence on the force-displacement curves of the dynamic term. Figure 3.13(a) shows that there are not any differences observed between the VPS explicit dynamic and the ABAQUS/Standard implicit static results. The use of a static code can reduce the computational times while giving accurate results.

Another modeling feature that would decrease the computational times, is the use of small-strain formulation instead of the large strain plasticity implemented in VPS. Simulations with this formulation can be computed with ABAQUS/Standard, however, the use of small-strain formulation does not present adequate results due to the large displacements observed in the normal tests as can be seen in Figure 3.13(b).

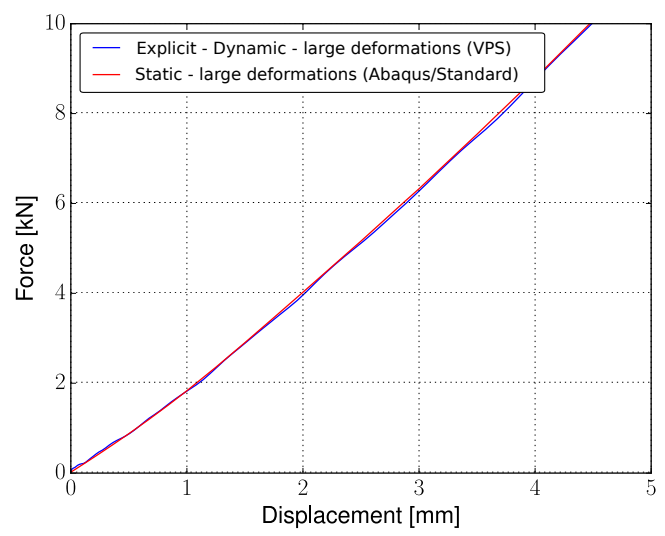

(a) Explicit-dynamic (VPS) vs. static code (ABAQUS/Standard).

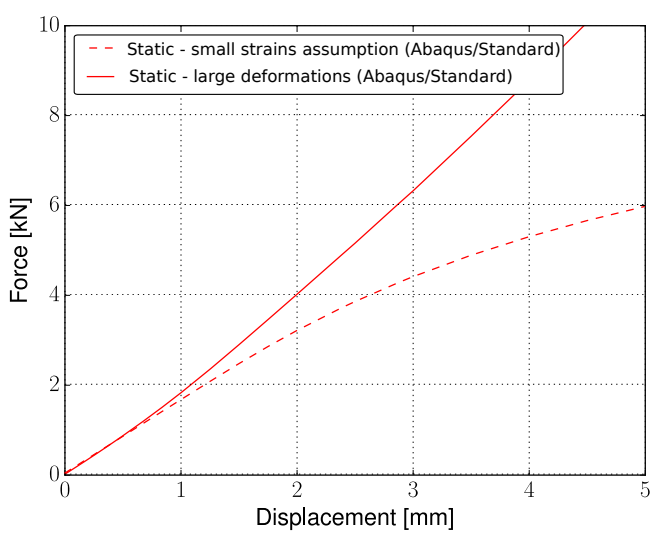

(b) Small-strains formulation vs largestrains plasticity.

Figure 3.13: Numerical analysis of spot weld model of normal test. 


\subsection{Preliminary results and loading cases}

Taking into account all the numerical features presented in the previous sections, some ilustrative preliminary results of the three loading tests simulations are presented in this section. The force-displacement results of the loaded welded specimens are shown in Figure 3.14(a) for the normal, shear and mix-mode loading cases applied to a joint of two sheets of $1.5 \mathrm{~mm}$ thickness.

Linear force-displacement curves with an extremely low slope are obtained for the normal test. The low slope can be explained by considering the measured displacements result mainly from the bending of the sheet surrounding the spot weld. Therefore, low forces should be applied in order to separate both sheets. Although these curves are commonly used to characterize a normal loaded spot weld, only the obtained maximum forces should be considered relevant to characterize the failure response in these tests, because the displacements are related with the surrounding sheet and not with the spot weld elongation.

Furthermore, for shear tests, the curves show a stiffer linear initial regime, followed by a non-linear regime that results from the plastic response of the material. These curves are mainly associated with the relative displacement between both joined sheets in the in-plane direction due to the spot weld deformations. The reached displacements are much lower than those examined in normal tests. It is observed, that despite the fact that the plastic zone is very confined around the notch tip (see Figure 3.7), the force-displacement curves lose the linearity after a relative small applied displacement.

Figure 3.14(b) represents the evolution of $J$-integral versus the applied force of the loading specimen for the different loading cases. It is well known that the forces applied in a normal direction in a notched geometry lead to higher stress concentration (and consequently $J$-integral values) than the forces applied in tangential direction. This phenomenon explains that the $J$-integral values obtained in the case of normal tests are higher than in the shear tests case for the same applied force magnitude. This higher $J$-integral values obtained for forces applied in normal direction explain the lower bearing capacity of joints loaded under these loading conditions.

The mix-mode test is simulated in the present chapter considering locked lateral displacements (taking into account the specimen position showed in Figure 3.4). Since the specimen is rotated forty five degrees when comparing with both pure normal and shear tests, both normal and tangentional components of total applied displacements 
have obviously the same magnitude. However, the loading specimen is much stiffer in the tangential direction due to the bending of the material in normal direction, and for the same applied displacement in both direction much higher resistance forces are obtained in tangential direction. These forces are extremely predominant in the total resistance force of the specimen, then the force-displacement curves of mix-mode tests are very similar to those of shear tests as observed in Figure 3.14. In a similar way, the higher tangential forces in the weld notch derived from this applied displacement component lead to a predominant fracture response associated to the mode II failure.

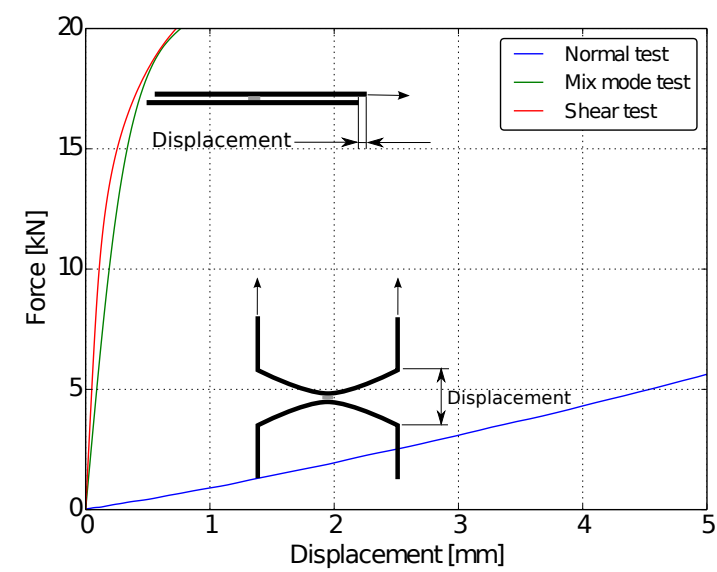

(a) Force displacement curves.

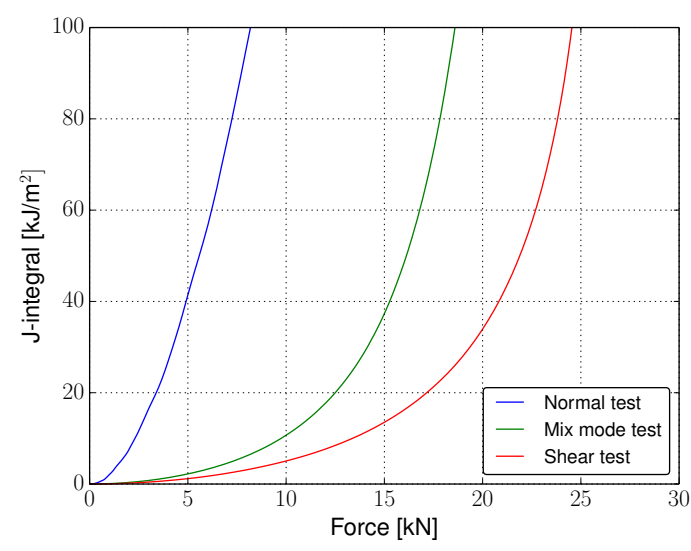

(b) J-integral evaluation.

Figure 3.14: Ilustrative simulation results of loaded spot weld at multiple angles.

\subsubsection{Critical notch section and crack advance direction}

There are two relevant sections in the joining specimen tested in this thesis, the cross section AA and the longitudinal section BB (see Figure 3.4). The preliminary simulations show that the critical section at which stress concentration, and therefore the $J$-integral, reaches higher values is Section AA for shear tests as observed in Figure 3.15(b), Notice that in these tests, the weld notch at section BB is loaded in pure mode II and at section AA is loaded in pure mode III (see fracture modes in Figure 2.11). On the contrary, Figure 3.15(a) shows that section BB is the most critical in normal tests. In these tests, the weld notch is loaded in mode I at all sections of the weld notch. In the case of mix-mode tests, the critical section is BB. Take note that in these tests, the applied displacements are a combination of 
those applied in the normal and shear tests: due to the normal applied displacement all weld notch sections are loaded at mode I; furthermore, because of the applied displacements parallel to sheet plane, section BB is loaded in mode II and section AA in mode III. The stress concentration induced by the applied shear displacements is higher than the stress intensities resulted from the applied normal displacements of the same magnitude. Therefore the mix-mode test are closer to the shear tests under the loading response point of view.

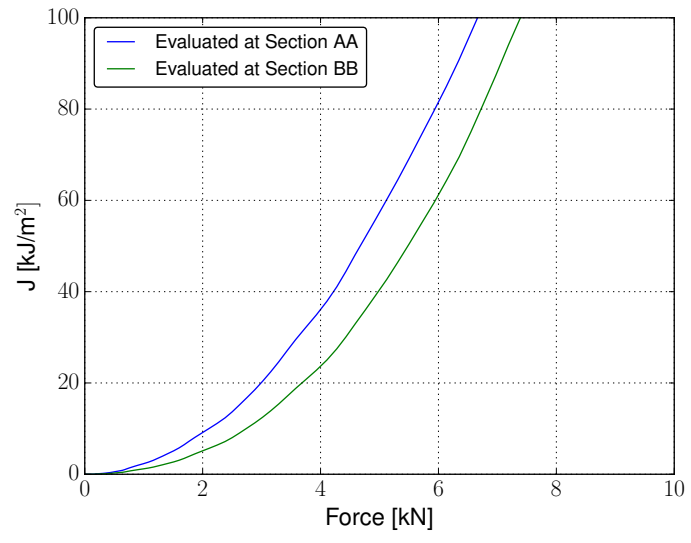

(a) Normal test.

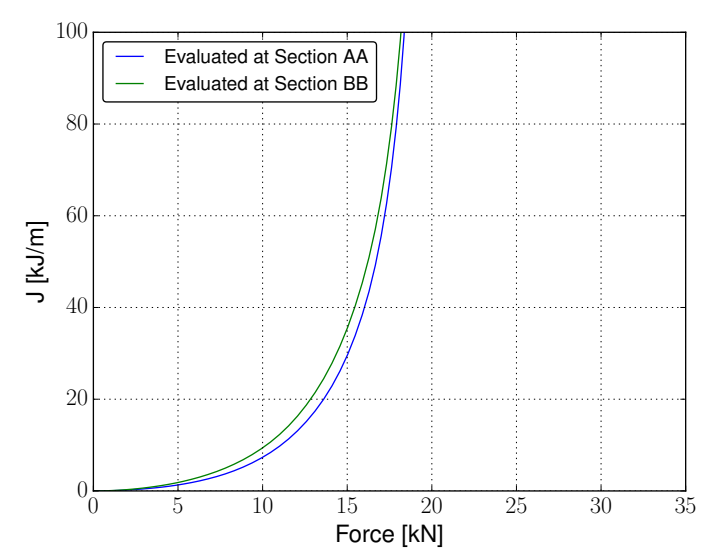

(b) Shear test.

Figure 3.15: J-integral evolution at different sections. 



\section{Chapter 4}

\section{Application in resistance spot welds of a hot formed steel}

The FEM model presented in Chapter 3 is validated in this chapter using experimental loading tests. The welded joints of a $22 \mathrm{MnB} 5$, martensitic boron steel, are used for this validation purpose. This steel, belonging to the AHSS family (see Figure 2.3), is extensively used in the automotive industry and has high mechanical properties after the hot forming process as presented in Section 2.2 .

A wide validation program is designed to cover the main variable's combinations which have an influence on the joints fracture response during a full-vehicle crash situation. The development of this experimental loading test campaign is presented in Section 4.1. The characterization of the spot weld features used for the FEM model are then presented. First, the geometrical features and metallurgical welding zones of the welds are identified in Section 4.2. The mechanical properties, such as the plasticity, of these zones are characterized in Section 4.3. The force-displacement curves and the the loading tests failed spot welds for all the combinations of the experimental campaign are presented and analyzed in Section 4.4. Finally, the experimental and the simulated results are compared in Section 4.5 for validation purposes. 


\subsection{Experimental validation campaign}

An experimental characterization and validation campaign has been developed in order to validate the FEM model for different conditions. First, the design of the text matrix considering the most relevant variables during loading and fracture response is presented. The welding process used for the multiple combinations is described.

\subsubsection{Test matrix design}

The experimental campaign must cover the main factors that have an influence on the joints fracture response during a real crash situation. The text matrix focuses on the three main factors controlling the joint fracture response: The sheet thickness combinations that are related to the crash-relevant structural components thickness, the loading angle associated with the local loading conditions of a joint during crash, and the spot weld diameter that depends mainly on the welding parameters and the sheet thickness combination. Other factors that can have an influence on the fracture response such as the loading speeds, the influence of the sheets' protection coat or the welding imperfections are not analyzed in this work. Finally, welds of three sheets are not considered not in the experimental campaign, an analysis of this type of joints can be found in Section 7.1 .

Three different sheet thicknesses have been considered in the present study: the minimum and maximum values currently used in the automotive industry regarding AHSS grades; $0.8 \mathrm{~mm}$ and $2.0 \mathrm{~mm}$, and an intermediate value of $1.5 \mathrm{~mm}$. All possible combinations between the three values are taken into account in order to characterize the influence of multiple thickness ratios. The resulting combinations are presented in Table 4.1 ,

The fracture response of joints strongly depends on the weld size and the weld size of a spot weld is given by some controlled factors such as the applied welding parameters and the sheet's thickness combination. However, it depends also on uncontrollable factors such as ambient conditions, electrode state or electrode contact with the sheets during welding. Usually, a set of welding parameters is developed to obtain a target weld diameter given by the welding standards depending on the sheet thickness combination. The target diameter of the joints used in this work follows the next formula: $d_{n}=4.5 \sqrt{t_{c}}$, where $d_{n}$ is the norm weld nugget diameter and $t_{c}$ is a measurement of both thickness combinations. Usually $t_{c}=0.2 t_{M}+0.8 t_{m}$ is used, 
4.1. Experimental validation campaign

\begin{tabular}{ccc}
\hline $\begin{array}{c}\text { Group } \\
\text { Number }\end{array}$ & $\begin{array}{c}\text { Sheet } 1 \\
\text { Thickness, } t_{1}(\mathrm{~mm})\end{array}$ & $\begin{array}{c}\text { Sheet } 2 \\
\text { Thickness, } t_{2}(\mathrm{~mm})\end{array}$ \\
\hline 1 & 0.8 & 0.8 \\
2 & 0.8 & 1.5 \\
3 & 0.8 & 2.0 \\
4 & 1.5 & 1.5 \\
5 & 1.5 & 2.0 \\
6 & 2.0 & 2.0 \\
\hline
\end{tabular}

Table 4.1: Sheets' thickness combinations used for the experimental loading campaign.

where $t_{M}$ is the maximum of both sheet thickness and $t_{m}$ is the minimum. However, these diameters cannot be obtained with high degree of precision and exactitude due to the uncontrolled factors. The actual joining diameters obtained in the joints of this work with the corresponding thickness combinations can be seen in Table 4.2 . Notice that the joining diameters are smaller than the weld diameters in the cases of joints with different thickness combination (see Figure 2.6.).

In order to evaluate the influence of the weld size on the fracture response of joints for a fixed thickness combination, the joints of one of the combinations (number 4 in Table 4.1) are tested using three different sets of welding parameters to obtain different welding diameters. Apart from the $d_{n}$ diameter joints, a greater $\left(d_{M}\right)$ and a smaller $\left(d_{m}\right)$ diameter are obtained and tested. More details about the welding parameters are presented in Section 4.1.2.

The bearing capacity of the spot weld joints depends on the local loading conditions that are applied to the joints. Among the multiple of loading tests used in the literature (see Section 2.4 to chracterize the joints fracture response, a joining specimen similar to KS-II specimen is used for the experimental campaign [36]. The two U-profiles are welded and loaded at three different angles between the sheets plane and the applied force: zero degrees to represent a pure shear test, ninety degrees representing normal loading conditions and forty-five degrees that represent mix-mode conditions (see Figure 3.4). The joints are loaded at these three angles for all the combinations presented in Table 4.1. The analysis of the influence of the spot weld diameter with the joints of combination 4 is only done for the normal and shear tests. The combination number six is not loaded at mix-mode tests. 


\begin{tabular}{|c|c|c|c|c|c|c|}
\hline \multirow{2}{*}{$\begin{array}{l}\text { Joint } \\
\text { Code }\end{array}$} & \multicolumn{2}{|c|}{ Sheet 1} & \multicolumn{2}{|c|}{ Sheet 2} & \multicolumn{2}{|c|}{ Diameter $(\mathrm{mm})$} \\
\hline & Mat & $t_{1}(\mathrm{~mm})$ & Mat & $t_{2}(\mathrm{~mm})$ & Target & Obtained \\
\hline U08U08n & $22 \mathrm{MnB} 5$ & 0.8 & $22 \mathrm{MnB} 5$ & 0.8 & $d_{n}=4.0$ & $3.60 \pm 0.2$ \\
\hline U08U15n & $22 \mathrm{MnB} 5$ & 0.8 & $22 \mathrm{MnB} 5$ & 1.5 & $d_{n}=4.4$ & $4.30 \pm 0.2$ \\
\hline U08U20n & $22 \mathrm{MnB} 5$ & 0.8 & $22 \mathrm{MnB} 5$ & 2.0 & $d_{n}=4.6$ & $4.00 \pm 0.2$ \\
\hline $\mathrm{U} 15 \mathrm{U} 15 \mathrm{~m}$ & $22 \mathrm{MnB} 5$ & 1.5 & $22 \mathrm{MnB} 5$ & 1.5 & $d_{m}<5.5$ & $4.00 \pm 0.2$ \\
\hline U15U15n & 22MnB5 & 1.5 & $22 \mathrm{MnB} 5$ & 1.5 & $d_{n}=5.5$ & $5.00 \pm 0.2$ \\
\hline U15U15M & $22 \mathrm{MnB} 5$ & 1.5 & $22 \mathrm{MnB} 5$ & 1.5 & $d_{M}>5.5$ & $6.00 \pm 0.2$ \\
\hline U15U20n & $22 \mathrm{MnB} 5$ & 1.5 & $22 \mathrm{MnB} 5$ & 2.0 & $d_{n}=5.7$ & $6.20 \pm 0.2$ \\
\hline U20U20n & $22 \mathrm{MnB} 5$ & 2.0 & $22 \mathrm{MnB} 5$ & 2.0 & $d_{n}=6.4$ & $5.90 \pm 0.2$ \\
\hline
\end{tabular}

Table 4.2: Sheet thickness combinations and corresponding target and obtained nugget diameter in similar 22MnB5 joints.

The AlSi coating that protects the 22MnB5 sheets against oxidation leads to an irregular weld notch (see more details in Section 4.2.2). The weld notch is a critical zone regarding spot weld fracture in AHSS as introduced in Section 3.6.1. Consequently, in order to analyze the AlSi coating influence on the fracture response, the U15U15M joints are tested as well using specimens at which AlSi coating is ground before welding. Only normal and shear tests are performed with these conditions.

Due to the uncertainties resulting from the welding process regarding spot weld geometry and the low repeatability of the maximum forces obtained for some cases in previous similar characterizations, the same tests are performed five times using the same conditions for each tested combination.

\subsubsection{Specimens welding}

The joining specimens are welded using a NIMAK C-type servo spot weld gun combined with a HWH control system. The main welding parameters are the applied electrode force, the applied current and the corresponding application times. For the joints tested in this section, the welding process of the scheme shown in Figure 4.1 is followed. The "squeeze time" $\left(t_{s q}\right)$ is necessary to ensure the required "applied electrode force" $(F)$. Two electrical pulses, the "weld current" $\left(I_{1}\right)$, and "temper current" $\left(I_{2}\right)$, are applied during the corresponding "weld time" $\left(t_{s 1}\right)$ and "temper time" $\left(t_{s 2}\right)$. Between these pulses, no current is applied during the so-called "cold time" $\left(t_{f 1}\right)$. During the "hold time" $\left(t_{f 2}\right)$, pressure is maintained after the heat cycle 
and it allows the molten material to solidify before releasing the welded sheet. The values of the welding parameters for each combination are shown in Table 4.3. In order to obtain the multiple diameters in combination $4\left(t_{1}=t_{2}=1.5 \mathrm{~mm}\right)$, different temper currents are applied. Higher currents than those presented in the Table 4.1 were applied in specimens of this thickness combination in order to obtain larger diameters. However, because welding splashes were observed during the welding process and these specimens have been rejected.

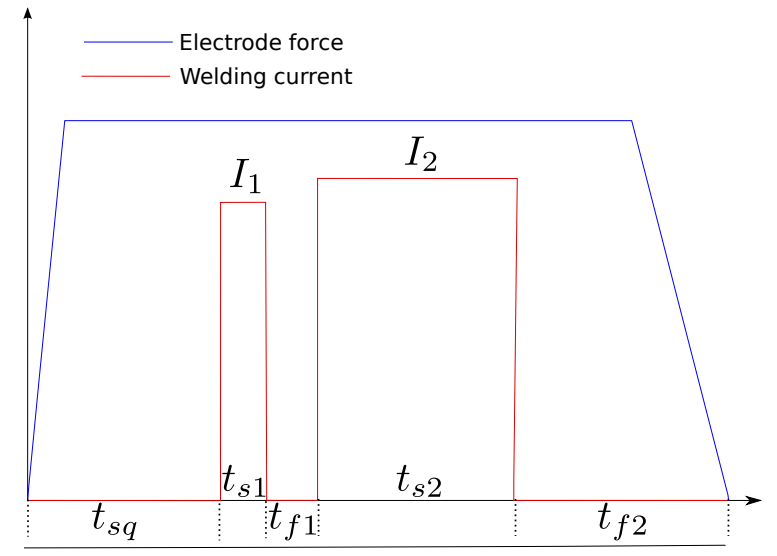

Figure 4.1: Overview of the welding process cycle used in this work for the similar joints of $22 \mathrm{MnB} 5$ steel.

\begin{tabular}{l|c|cc|ccccc}
\hline Joint & Force $(\mathrm{kN})$ & \multicolumn{2}{c}{ Intensities $(\mathrm{kA})$} & \multicolumn{5}{c}{ Time $(\mathrm{ms})$} \\
Code & $\mathrm{F}$ & $I_{1}$ & $I_{2}$ & $t_{s q}$ & $t_{s 1}$ & $t_{f 1}$ & $t_{s 2}$ & $t_{f 2}$ \\
\hline U08U08n & 3.00 & 3.00 & 4.80 & 1000 & 200 & 20 & 400 & 200 \\
U08U15n & 3.00 & 3.00 & 5.00 & 1000 & 200 & 20 & 400 & 200 \\
U08U20n & 3.00 & 3.00 & 4.80 & 1000 & 200 & 20 & 400 & 200 \\
U15U15m & 3.80 & 3.00 & 4.30 & 1000 & 200 & 20 & 400 & 200 \\
U15U15n & 3.80 & 3.00 & 5.20 & 1000 & 200 & 20 & 400 & 200 \\
U15U15M & 3.00 & 3.00 & 6.20 & 1000 & 200 & 20 & 400 & 200 \\
U15U20n & 4.00 & 3.00 & 5.80 & 1000 & 200 & 20 & 400 & 200 \\
U20U20n & 3.00 & 3.00 & 5.40 & 1000 & 200 & 20 & 400 & 200 \\
\hline
\end{tabular}

Table 4.3: Welding parameters for all thickness and target diameter combinations in similar 22MnB5 joints. 


\subsection{Geometry and local material properties distribution of the spot welds}

The geometry and the material properties distribution of the spot welds are analyzed in order to characterize the detailed FEM model. The spot weld geometry and dimensions along with the welding metallographic zones considering the combinations presented in Table 4.2 are analyzed using cross sectioned spot welds. Hardness line measurements are performed in the cross sectioned spot welds in order to identify the main metallographic zones and their dimensions.

\subsubsection{Methodology}

In order to analyze the geometry and the welding zones of the welds, macroscope metallographic observations of cross sectioned spot welds are performed. For this purpose, small samples are welded and cross sectioned. The samples consist of two $45 \times 45 \mathrm{~mm}^{2}$ flat sheets which are extracted from larger flat sheets. In order to avoid irregularities caused by the hot forming process the extraction operation stays away from the larger sheet's edges. After being welded, they are carefully cross sectioned along a symmetry plane and mounted in epoxy resin. They are ground, polished and chemically etched. Chemical etching is Nital $1 \%$ that reveals ferrrictic and martensitic microstructures. Micro-hardness measurements are taken following a line crossing from the top sheet to the lower sheet and passes the nugget (see Figure 4.5). The measurements are performed using a $0.1 \mathrm{~kg}$ load following the Vickers settings and are spaced approximately $0.5 \mathrm{~mm}$.

The analyzed sheet thickness and material combinations are those used for the loading tests presented in Table 4.1. Combination number four is also analyzed without the AlSi coating, in order to determine the influence of this coating in the weld geometry and microstructure. This coating is removed after the hot stamping process and before welding. The same sets of welding parameters presented in Table 4.3 for the loading test are used for these specimens.

\subsubsection{Cross sections and geometry analysis}

Figure 4.2(a) shows a typical cross sectioned spot weld of a 22MnB5 steel with both sheets of the same thickness $(1.5 \mathrm{~mm})$. The contour of the fusion zone, also called 
fusion line, can be identified clearly. It can be observed that the fusion zone is situated in the middle of both sheets. No welding imperfections can be seen in this cross section. The limits of the different heat affected zones that surround the fusion zone are also noticeable. A spot weld of the same thickness combination in which AlSi coating has been removed before welding is shown in Figure 4.2(b). Take note that whether with or without the AlSi coating, the same fusion zone and HAZ shapes can be observed. Detailed micro photographs of both weld notch samples are shown in Figure 4.3, and an high irregular shape can be observed in the case of coated sheets. Remains of AlSi are retained in the limits of the weld and may introduce brittle paths where the crack may begin during loading. Micro cracks are observed as well in the uncoated weld notch.

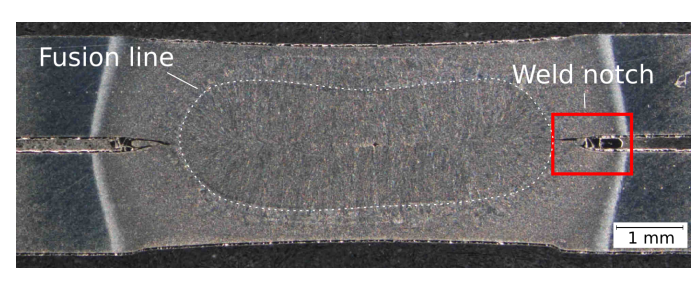

(a) With AlSi coat.

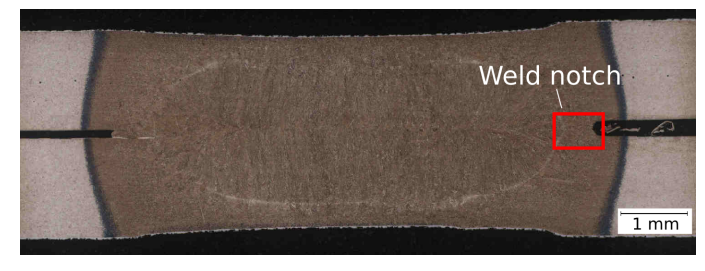

(b) Without AlSi coating.

Figure 4.2: Cross sectioned spot weld general views. The joints have the same material and thickness combination of $22 \mathrm{MnB} 5$ and $t_{1}=t_{2}=1.5 \mathrm{~mm}$.

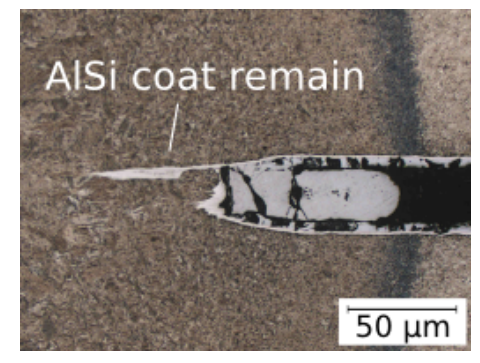

(a) Usual joint.

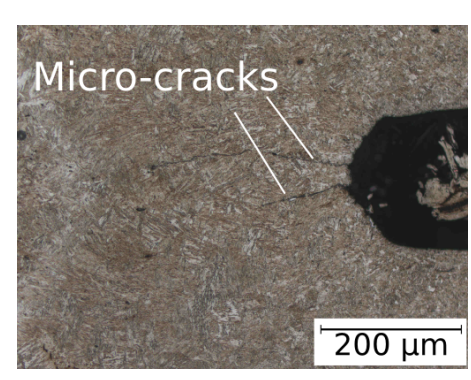

(b) Without AlSi coating.

Figure 4.3: Detailed image of the weld notch of 22MnB5 similar joints of Figure 4.2

A cross sectioned spot weld of a joint of two $2.0 \mathrm{~mm}$ sheets is presented in Figure 4.4(a). A similar nugget fusion line as the one observed for the combinations of $1.5 \mathrm{~mm}$ sheets can be see here. However, a welding void can be found in the middle of the nugget. It can be explained by considering the high thickness values, that leads 
to some difficulties during the complete solidification of the fusion zone resulting in solidification voids. These voids may have an influence on the joints loading behavior and they cannot be introduced in the detailed FE models.

Figure 4.4(b) shows a cross section of a joint with very different thickness combinations, i.e. one thick sheet of $2.0 \mathrm{~mm}$ and one thin sheet of $0.8 \mathrm{~mm}$. In these types of combinations, the nuggets long axis is located in the middle point of both sheets, displaced from the faying surface. The top border of the fusion zone is close to the weld notch tip. This phenomenon would lead to the Partial or Total Dome failure shown in Figure $4.20(\mathrm{c})$.

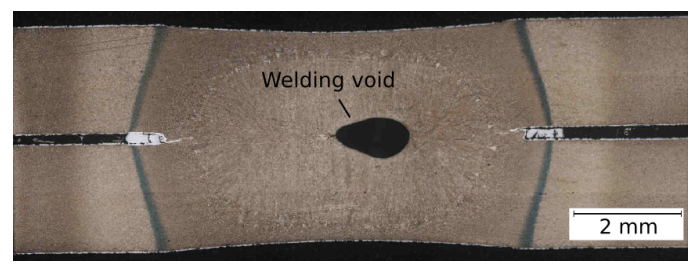

(a) U20U20n.

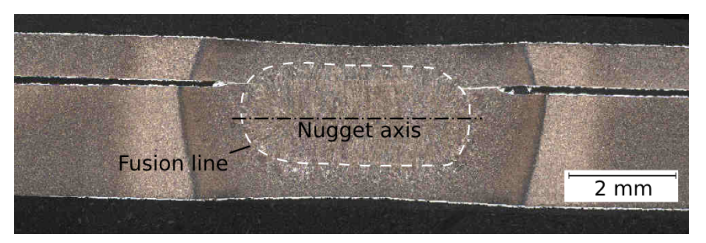

(b) U08U20n.

Figure 4.4: General view of a cross sectioned spot weld general with dissimilar thickness combination.

\subsubsection{Metallography and hardness profile}

The hardness profile of a joint is presented in Figure 4.5. On the right side of the figure a simplified scheme neglecting the hardness measurements scattering effects is represented. Three zones are distinguished from the hardness point of view: (i) The FZ and the so-called hard HAZ HHAZ with a roughly uniform martensitic hardness (480-520 HV), (ii) the so-called soft HAZ SHAZ with hardness decreasing until almost $300 \mathrm{HV}$, and the base material BM with the typical hardness of a fully hardened $22 \mathrm{MnB} 5$, i.e. $500 \mathrm{HV}$. The same profile is observed for all combinations, only differences in the dimensions of each zone are observed, i.e., the radii that represent the FZ, SHAZ and HHAZ dimensions $\left(\mathrm{r}_{1}, \mathrm{r}_{2}\right.$ and $\left.\mathrm{r}_{3}\right)$ are lineally dependent on the spot weld radius. This hardness profile can be explained by observing detailed micrographs of the metallography in different zones. The main welding zones are distinguished in the general optical micrograph of Figure 4.6. In addition, it must be pointed out that a confined hardness drop can be seen in some coupons in a extremely localized zone at the fusion zone boundary. This phenomenon has already been reported by other 
authors and that has attributed it to phenomenological changes in the microstructure of the fusion line. This phenomenological change is probably caused by the lower cooling rates due to carbon conditioned effusion from the fusion line towards the fusion zone [99].

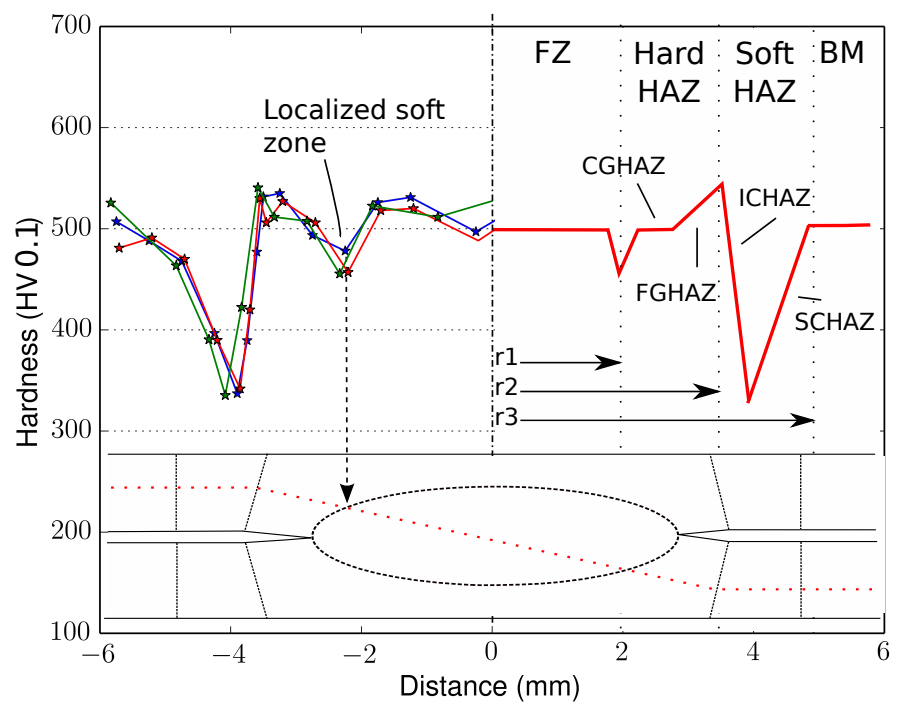

Figure 4.5: The HV profile of a similar 22MnB5 steel joint (U15U15n). Left: Hardness measurements. Right: Scheme of the profile with the main zones.

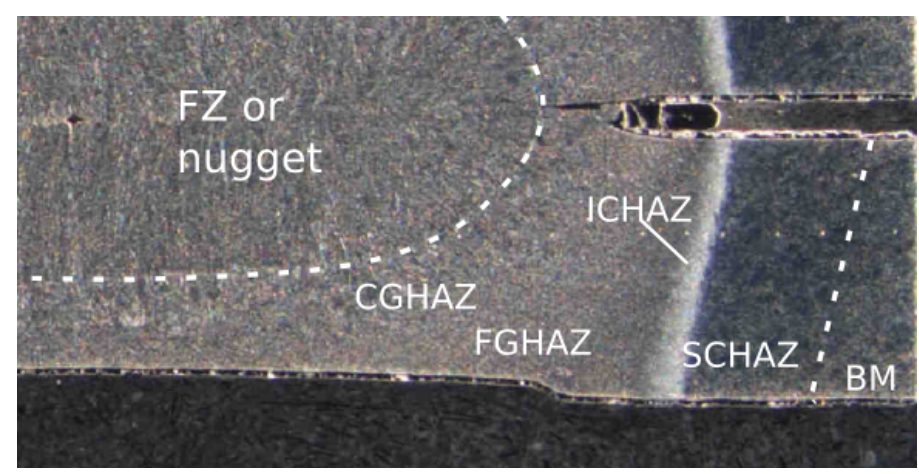

Figure 4.6: An optical micrograph of the fully hardened 22MnB5 similar spot weld cross section. The multiple welding zones that are shown are: (i) The nugget or fusion zone (FZ), the heat affected zone (HAZ) and (iii) the base material (BM). HAZ is subdivided in the so-called fine grain HAZ (FGHAZ), the coarse grain HAZ (CGHAZ), the intercritical HAZ (ICHAZ), and the subcritical HAZ (SCHAZ). These zones are analyzed in detail in the micrographs presented in Figure 4.7. 

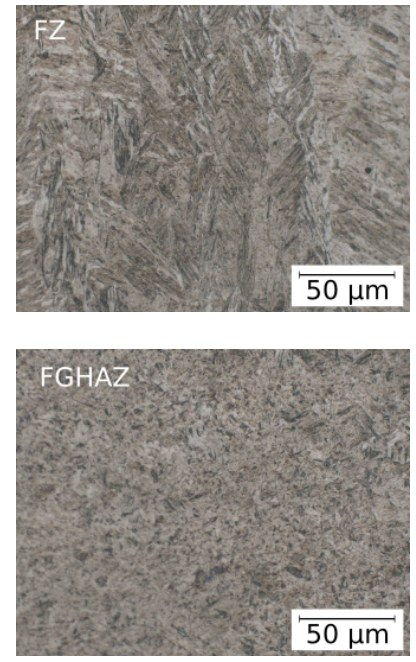
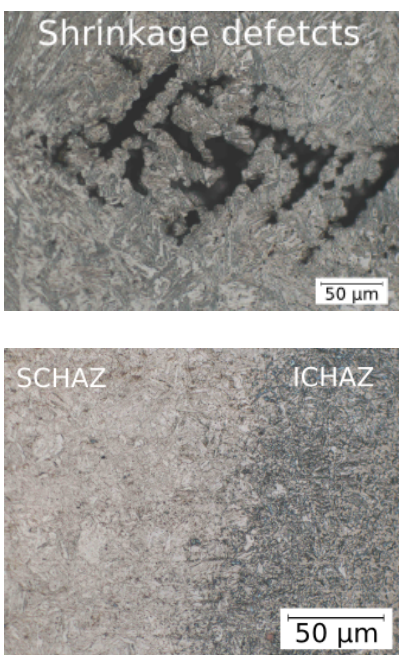
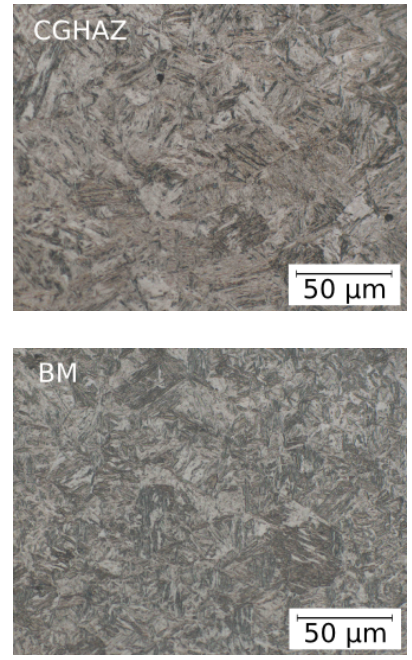

Figure 4.7: The optical micrographs of multiple spot weld zones of a similar 22MnB5 joint. From top left to bottom right: FZ presents a martensite phase due to rapid cooling at which martensitic microstructures showed directional, columnar solidification from the nugget boundary (first) towards the center (last). Some shrinkage defects can be observed in the center of the fusion zone of some spot welds. FGHAZ and CGHAZ are the closest zones to FZ, where the material temperature has increased above $\mathrm{Ac}_{3}$ and has been completely transformed into austenite leading to martensitic microstructure after rapid cooling. CGHAZ has staid longer than FGHAZ above $\mathrm{Ac}_{3}$ leading to coarser martensitic grains. FZ and CGHAZ are gathered in the Hard HAZ (HHAZ) under the hardness values, and therefore material properties, point of view. The next layer is the ICHAZ, at which temperatures were above $\mathrm{Ac}_{1}$ without reaching $\mathrm{Ac}_{3}$. It caused the partial transformation of base material into austenite and stable ferrite. After cooling, the austenite phase was changed into martensite again. Resulting microstructure consists of martensite and some ferrite phase. This area has less than $100 \mu \mathrm{m}$ of thickness. In SCHAZ, temperatures were bellow $\mathrm{Ac}_{1}$, changing microstructure into tempered martensite. ICHAZ and SCHAZ are grouped in the Soft HAZ (SHAZ). Finally the BM has a typical martensitic of the fully hardened $22 \mathrm{MnB} 5$.

In summary, a weld is divided in two zones from the hardness and corresponding material properties point of view, on the one one FZ and HHAZ with high hardness due to the martensitic microstructure, and on the other hand, a SHAZ with lower hardness due to ferrite phases and tempered martensite and a martensitic BM with high hardness. For modeling spot weld behavior BM and FZ/HHAZ are considered extremely similar. Tensile tests used to validate this assumption are presented in Section 4.3 . 


\subsection{Characterization of nugget material properties}

The metallography and hardness analyses of $22 \mathrm{MnB} 5$ spot welds that have been presented in the previous section have shown that, apart from the HAZ, the mechanical properties of the nugget should be characterized in order to have a detailed plasticity model of all spot weld zones of the material presented in this chapter.

For this purpose, micro tensile specimens specially designed to have nugget material in the gauge zone are loaded up to fracture [15, 16, 58, 100]. The obtained force-displacement curves of these tests are compared with those obtained from nonwelded specimens of the same geometry. Two specimen geometries are used, they are named according to the loading conditions that they represent, uniaxial tension (UT) and plane strain tension (PST). Figure 4.8 shows the geometries and the dimensions of both specimens that have been extracted from a previous Gumbsch et al. work [101].

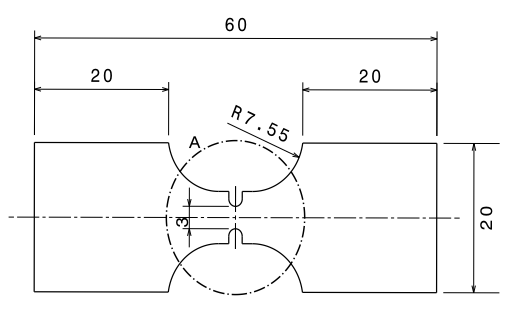

(a) PST specimen.

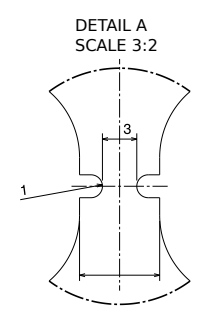

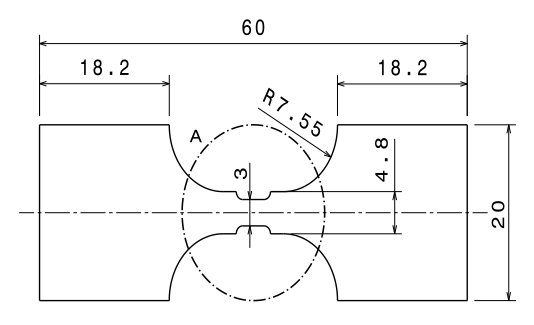

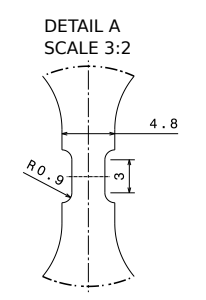

(b) UT specimen.

Figure 4.8: Reduced tensile tests specimens geometries. The small gauge zone where high strains and fracture occur have fusion zone properties in the welded specimens.

\subsubsection{Specimens production}

In order to obtain fusion zone martensitic material in the gauge zone, where plastic strains occur during loading, micro tensile specimens are extracted from welded plane sheets. For this purpose, sacrificial coupons of $1.5 \mathrm{~mm}$ thickness are welded onto $130 \mathrm{x}$ $60 \times 1.5 \mathrm{~mm}$ coupons using the welding parameters depicted in Tables 4.3 for the $t_{1}=$ $t_{2}=1.5 \mathrm{~mm}$ thickness combination. The sacrificial plates are then carefully separated with an electro discharge machining (EDM) wire, which is a suitable method for this application, as the thin wire can get in between the two welded sheets and cut through 
the hard nugget without damaging the surrounding HAZ. EDM is also used to cut the coupons to produce the geometries of the UT and PST specimens. After the extraction of the sacrificial coupons and cutting the specimens, they are ground on both sides, in order to eliminate the electrode indentation, the irregularities of the fusion zone surface and the AlSi coat. A thickness of $0.15 \mathrm{~mm}$ is ground from the side of faying surface, and a $0.35 \mathrm{~mm}$ thickness is extracted from the electrode indentation side. The resulting specimens have $1 \mathrm{~mm}$ thickness and no AlSi coating. A scheme of the aforementioned procedure can be seen in Figure 4.9 .

\section{a}

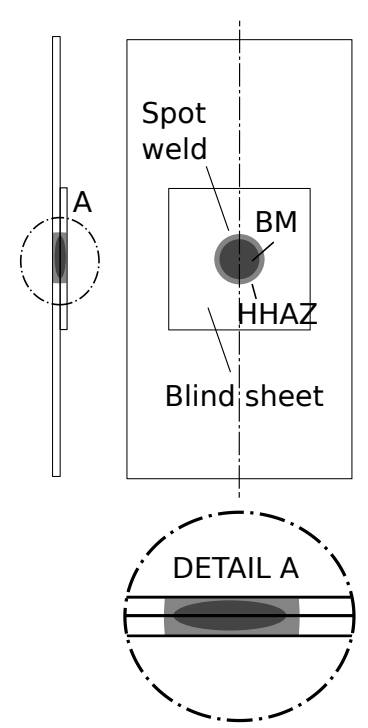

b

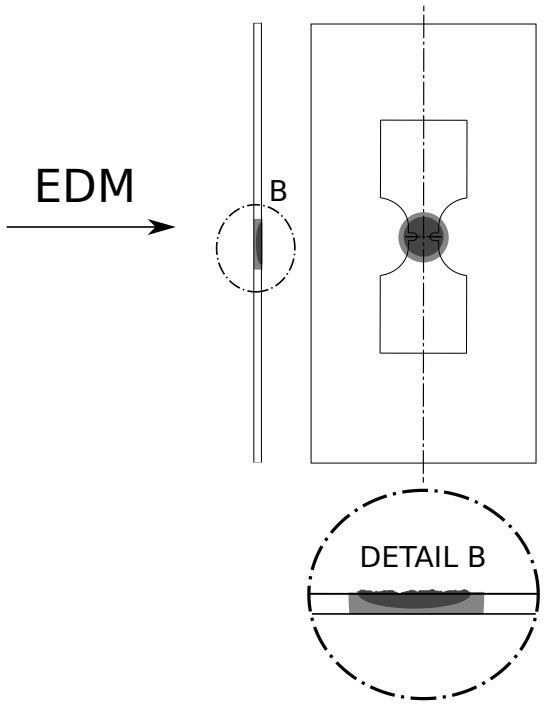

C
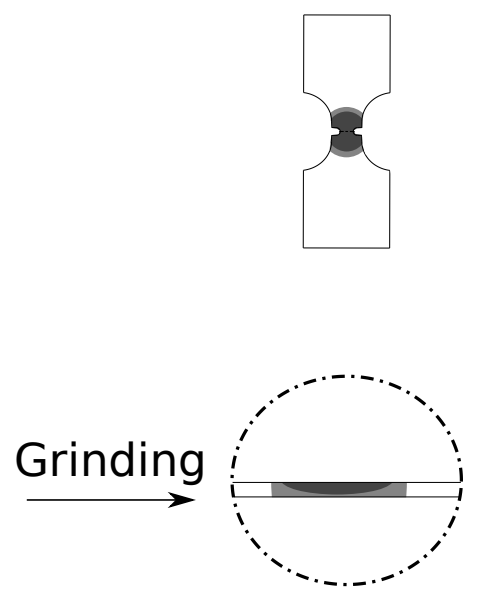

Figure 4.9: Scheme of the process followed to obtain the micro specimens.

[Scheme of the process followed to obtain the micro specimens, from left two right:

(a) Two plane coupons are welded, (b) The micro specimens are cut and the sacrificial coupon is removed using EDM and (c) the micro specimens are ground.]

After the aforementioned procedure, a spot weld of around $6 \mathrm{~mm}$ diameter is located in the center of the specimens. The shoulders of the specimens allow the softened HAZ of the specimens to have a larger section. This then leads to strain concentration and fracture only in the weld material during loading. Same specimens of non-welded fully hardened material are tested. These coupons have the same geometry as the welded ones, and they are ground as well to obtain uncoated specimens and the same thickness $(1 \mathrm{~mm})$. Five tests of each geometry are then performed to check the repeatability. 


\subsubsection{Mechanical tests}

A $250 \mathrm{KN}$ Zwick Roell universal material testing machine is used to load the specimens with a small load cell of $10 \mathrm{kN}$. The extensions are measured using the DIC system. A gauge length $L_{0}=4 \mathrm{~mm}$ is used for the PST specimen and $L_{0}=6 \mathrm{~mm}$ for the UT specimens. These two points are situated on the sides of the zone where the plastic strains are developed. The measured displacements between both points are used for the force-displacement curves. These displacement's measurements are performed using a dedicated digital image correlation (DIC) algorithm.

Figure 4.10 shows the most representative force-displacement curves of welded and unwelded specimens for both tested geometries. Similar results are observed between welded and unwelded specimens for both geometries. Only slight differences are observed in some tests in PST tests, probably due to sliding effects between specimens and the clamping device. These results lead to the conclusion that the plastic strain evolution is the same for those specimens with nugget material in the gauge zone and those with fully hardened 22MnB5 base material. Consequently, the assumption that BM, HHAZ, and FZ have identical mechanical properties (and therefore same plasticity constitutive model) is validated. The same hardening curves used for the fully hardened 22MnB5 base material can be used in the plasticity model of the HHAZ and the FZ of the spot weld, during the detailed spot weld failure modeling.

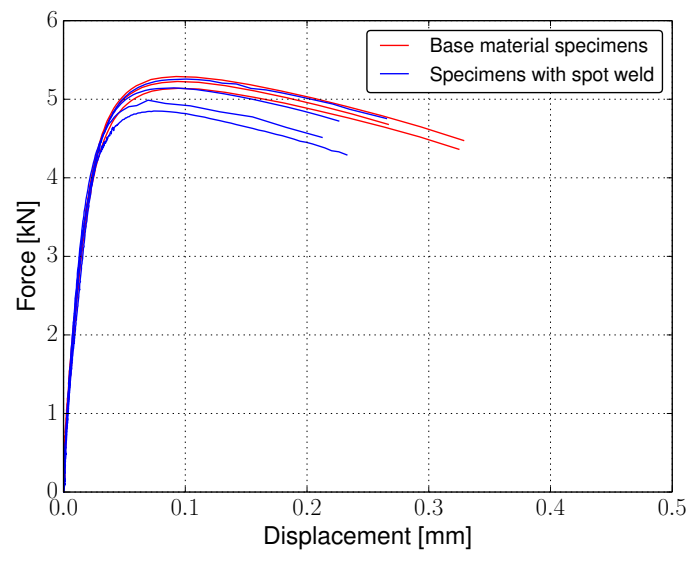

(a) PST tests.

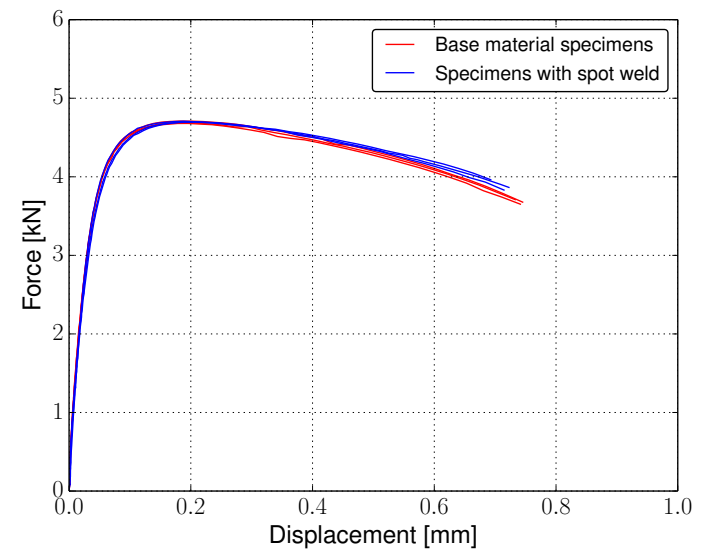

(b) UT tests.

Figure 4.10: Reduced tensile 22MnB5 tests with and without weld material in the gauge zone. 


\subsection{Experimental loading tests of the joining specimens}

The experimental campaign introduced in Section 4.1 to evaluate the bearing capacity of the joints are presented in this section. The production of the specimens as well as the loading tests are explained in Section 4.4.1. The force-displacement curves of all tested combinations are presented in Section 4.4.2. The main features are reported in order to reproduce the exact boundary conditions during the FE modeling of spot weld loading and francture. For this reason, a detailed analysis of the fracture of these loading tests is given in Section 4.4.3.

\subsubsection{Preparation of U-profiles and loading tests of welded joints}

U-profiles are used to produce the joining specimens of loading tests. These Uprofiles are extracted from flat sheets using a hot forming process where 22MnB5 with fully hardened grade properties is obtained. Five halves of the joining specimens are extracted from each long U-profile using laser cutting technology.

Both halves of the joining specimens corresponding thicknesses are welded using the procedure presented in Section 4.1.2. Then, the resulting joined specimens are loaded using a universal Zwick Machine with a $250 \mathrm{kN}$ cell. A device specially designed for this purpose is used to apply the force from different loading angles. The

applied displacement velocities controlled by the Zwick machine are $10 \mathrm{~mm} / \mathrm{min}$ for normal tests, $5 \mathrm{~mm} / \mathrm{min}$ for mix-mode tests and $2 \mathrm{~mm} / \mathrm{min}$ for shear tests.

The displacements of both sides of the specimens are measured using DIC as well as Zwick machine traverse measurements. DIC system allows measuring the displacements directly in the specimen using images that are recorded during the test.

\subsubsection{Force-displacement curves}

Figure 4.11 shows the shear tests force-displacement curves. Figure 4.11(a) displays curves of the most representative test for each multiple thickness combinations. At first glance, a strong dependency of the maximum forces on the thickness combination is apparent. However, the curves of Figure 4.11(b), where tests with constant thick- 
ness combination and multiple weld sizes, show that the maximum force is extremely sensitive to the spot weld diameter. Therefore, the maximum forces variation in Figure 4.11(a) is caused mainly by the differences in the spot weld diameter obtained for each thickness combination (see Table 4.2). In addition, it must be noticed that in Figure 4.11(b) the results of three tests for each weld size are plotted and have high repeatable curves witch can be observed both for the maximum forces and the loading curves shapes.

All the curves of Figure 4.11 are plotted using displacements measured by the Zwick machine in the traverse. However, in Figure 4.11(a), the curves of two combinations (U08U15n and U15U20n) are plotted using the displacement measured by DIC system in the clamping device, in other words the actual relative displacement between both sheets. The DIC displacements are lower, because Zwick traverse also measures the stretching of the machine and the small sliding displacement between specimen and clamping device are included. For validation purposes of the FEM model, the DIC measurement should be used.

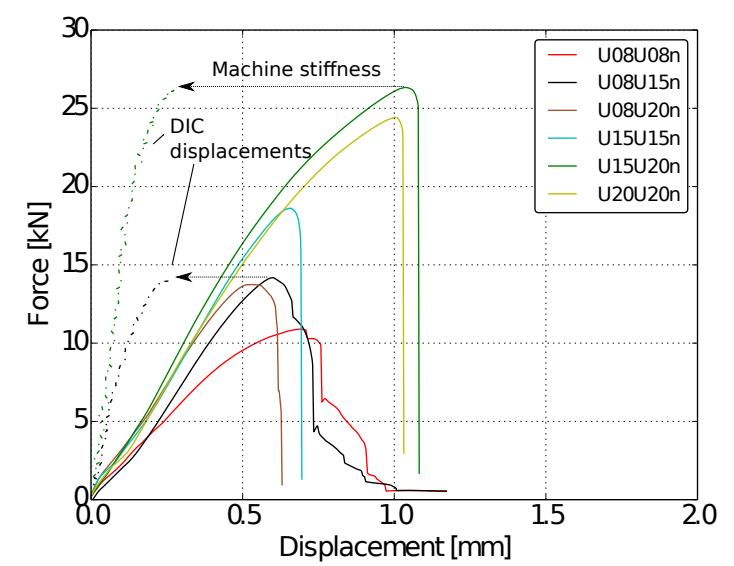

(a) Multiple thickness combinations.

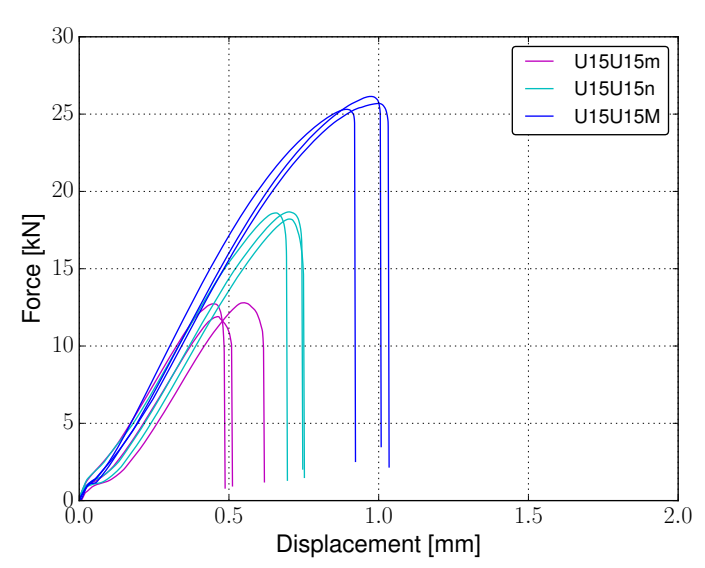

(b) Different spot weld diameters.

Figure 4.11: Force-displacement curves of shear tests for similar 22MnB5 joints.

The force-displacement curves of normal tests are shown in Figure 4.12. The curves of the most representative test of each thickness' combination are plotted in Figure 4.12(a). Notice that the large measured displacements result from the sheet bending around the weld during loading (see Figure 3.14(a) ). This is the reason why the slopes of the force-displacement curves increase with the thickness combination. On the one hand, differences in the maximum forces are observed between the mul- 
tiple sheet combinations. On the other hand, the loading tests results of joints with different weld diameters (maintaining the thickness combination) are presented in Figure $4.12(\mathrm{~b})$. In this case, the curves of three tests are plotted for each welding diameter. Slight differences can be observed between the tests of different weld sizes regarding the maximum forces reached, however the differences are much lower than in the case of shear tests. Some scattering can be observed for each weld diameter regarding the onset of maximum forces. Notice that in the case of U15U15M, the maximum forces are higher. However, take note of the strong force drop observed earlier during loading. This force drop results from the first crack advance in the joint, and it is considered the critical maximum force in this thesis. More details about this phenomenon is given in Section 4.4.3. There are slight differences in the loading slope and they are attributed to the low repeatability of the experimental set up conditions.

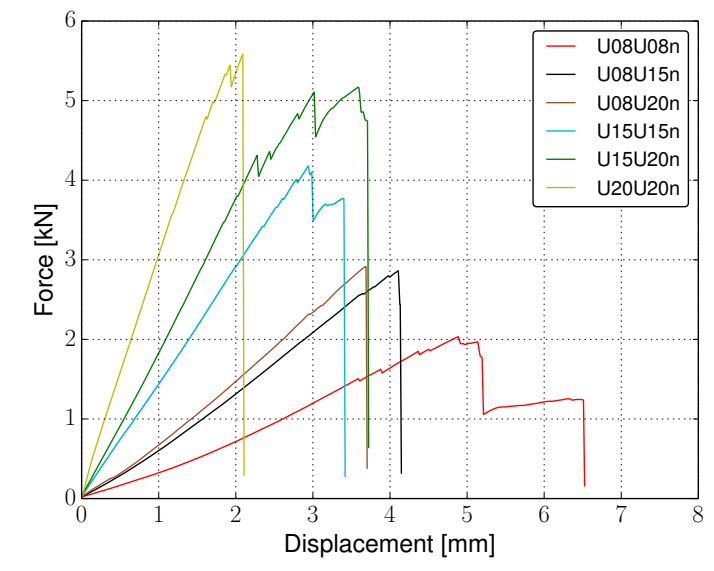

(a) Multiple thickness combination.

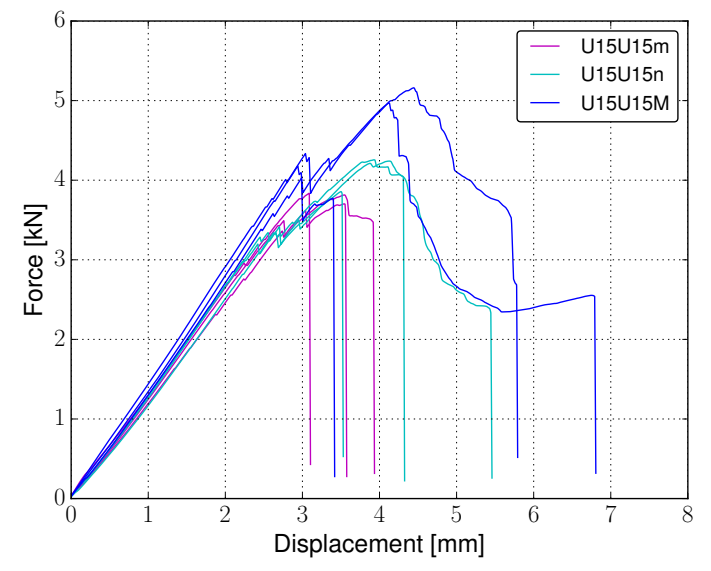

(b) Different spot weld diameters.

Figure 4.12: Force-displacement curves of normal tests for similar 22MnB5 joints.

Figure 4.13 shows the mix-mode tests force-displacement curves for multiple thickness combinations specimens. In Figure 4.13(a) the results of three repetitions are presented for each combination presenting high repeatability. Notice that in these tests, the total applied displacements are a combination of displacements in both directions, i.e. in parallel direction and normal direction. Consequently, the phenomena that can be found for the both pure tests are observed. Due to the experimental set up conditions, lateral displacements are not completely restricted. Therefore, the lower resistance of the loading specimen to normal displacements (compare slopes of 
Figure 4.11 and Figure 4.12, the both sides the specimens tend to separate from each other in the normal direction leading to lateral displacements regarding the 45 degrees position (see Figure 3.4). These lateral displacements are experimentally measured with the DIC device. In addition, the joints where one of the sheets is very thin, i.e. $t=0.8 \mathrm{~mm}$, present much lower maximum forces due to the lower weld diameter. A low force drop can be seen in some curves in Figure 4.13(a), It derives from specimens sliding from the grips, and it is not observed if the DIC displacements are plotted as in Figure 4.13(b).

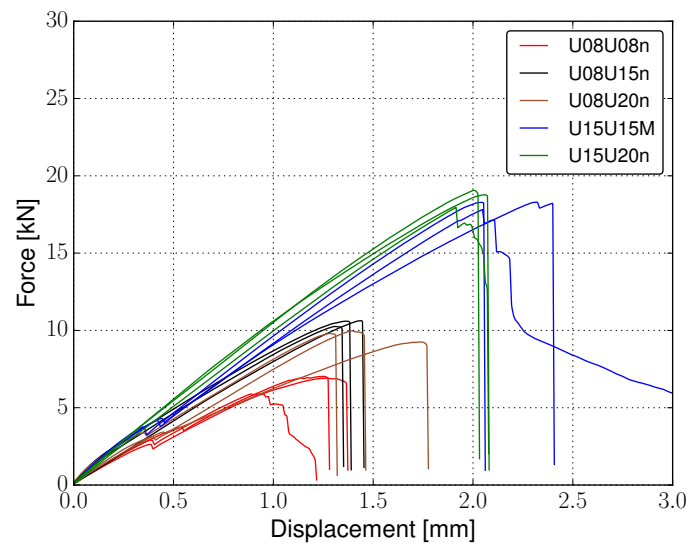

(a) Zwick displacements.

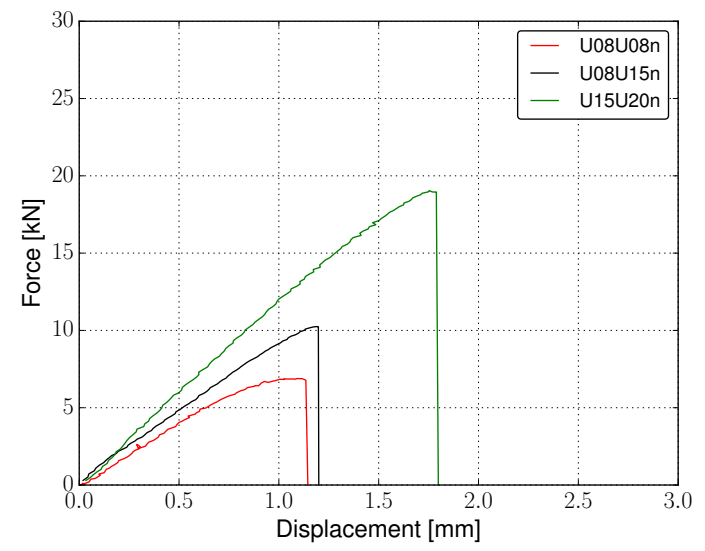

(b) DIC displacements.

Figure 4.13: Force-displacement curves of mix-mode tests for similar 22MnB5 joints and multiple thickness combinations.

The influence of the AlSi coating on the loading and fracture response is presented in Figure 4.14. Despite the differences observed in notch tip geometry (see Figure 4.2, slight differences are observed in the force-displacement curves between coated and uncoated sheets, both for normal and shear tests. The slightly higher maximum forces presented in the shear tests are caused by the higher welding diameters obtained in uncoated sheets, taking into account that the same welding parameters are used for all specimens but the ausence of AlSi coating leads to higher melting current during welding and consequently larger weld sizes. High scattering is observed in the onset of crack forces for all normal tests. Therefore, unclear conclusions can be extracted in these tests.

Finally, Figure 4.15 shows shear tests force-displacement curves of the same thickness combinations $\left(t_{1}=t_{2}=0.8 \mathrm{~mm}\right)$ and welding parameters, but presenting dif- 


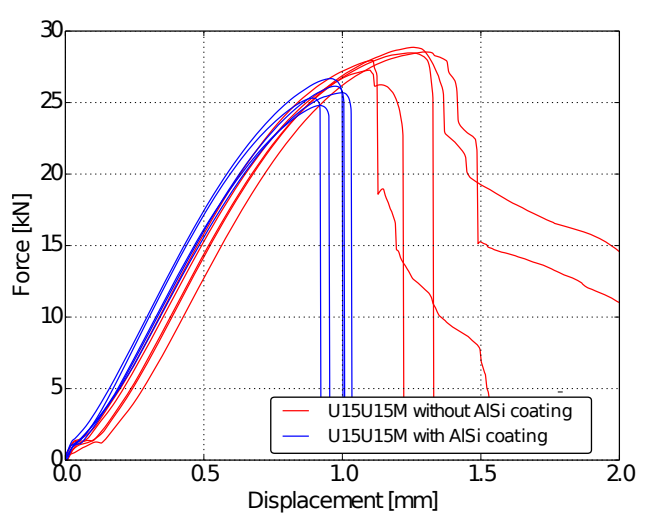

(a) Shear tests.

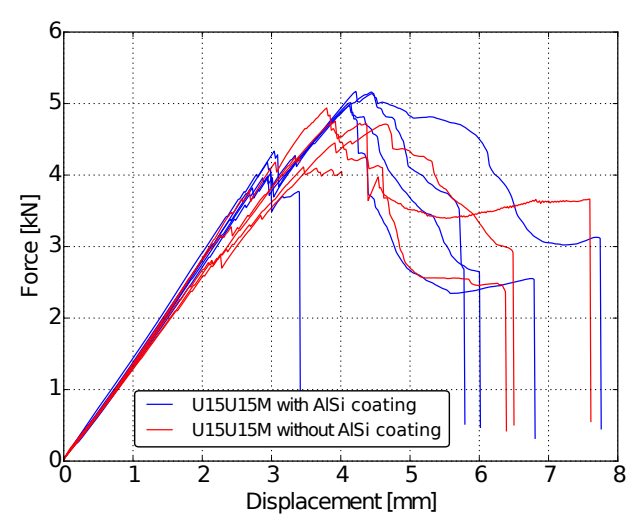

(b) Normal tests.

Figure 4.14: Force-displacement curves of tests with specimens of 22MnB5 with or without AlSi coating.

ferent maximum forces. After a detailed observation of the failed experiments, it has been found that those specimens with lower maximum forces are those with welded splashes around the joint. This phenomenon can be explained when looking the weld diameters. The joints with splashes present lower diameters, because some melted material is expelled from the joint during welding. This lower diameter leads to lower maximum forces due to the strong sensitivity of these tests to weld sizes. Some splashes have been observed in the specimens on some normal tests, however due to the low sensitivity to weld diameters in this kind of test no differences in the force-displacement curves have been observed.

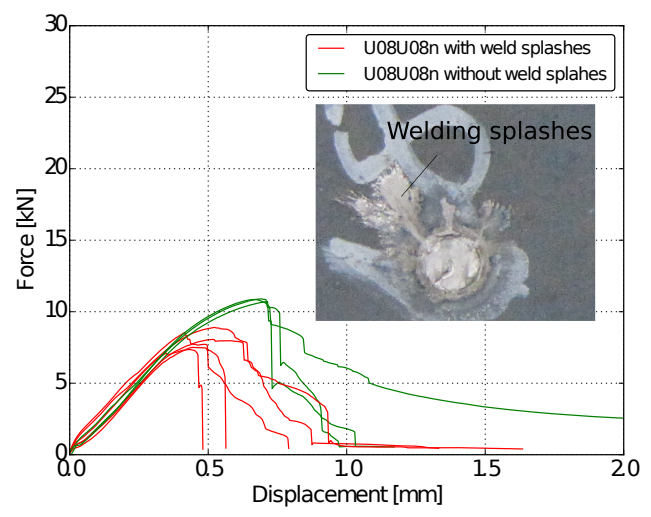

Figure 4.15: Force-displacements curves of shear tests with and without welding splashes. Lower maximum forces are obtained in those tests where welding splashes were detected. 
4.4. Experimental loading tests of the joining specimens

\subsubsection{Fracture analysis}

Observing the force-displacement curves and fractured surfaces two fracture mechanisms can be recognized in the tested welds: (i) brittle fracture through the nugget or along the FZ boundary in shear and mix-mode tests, and (ii) ductile fracture through the nugget in case of the normal tests.

An unstable crack advance that splits the nugget into two pieces after the onset of the crack is observed in shear tests and it leads to brittle mode II failure of the joint. A brittle surface can be observed after failure and the force-displacement curves of the tests drops to zero drastically after crack nucleation (see Figure 4.11). The same behavior can be observed in the mix-mode tests, with brittle force-displacement curves and brittle failure surfaces. The shear component of the applied displacements is predominant at this loading angle leading to mode II brittle failure.

In the case of normal tests, the crack advances in a stable way. First, weld notch blunting occurs, followed by crack nucleation and finally crack propagation (see Figure 4.16). After crack nucleation higher forces have to be applied in order to propagate the crack through the material. The crack has in some cases a complex three dimensional propagation path, as can be observed in the micro CT images of the interrupted tests of Figure 4.17, following the weakest path in the FZ material. This path depends on the specific FZ imperfections, leading to non-repeatability of the failed spot weld final appearance. In some cases, the crack crosses the fusion completely and in other cases it turns and advances until the outer surface of one of both sheets (see an example in Figure 4.18). As a result, force-displacement curves after the crack nucleation are not repeatable. From an industrial point of view, the crack evolution is not critical, and the joint is considered to have failed after the crack nucleation is signaled by the first force drop in the force-displacement curve.

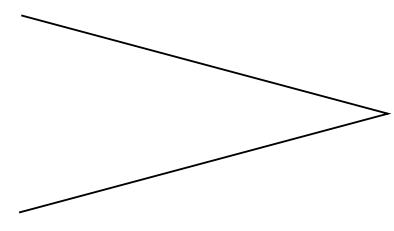

Original weld notch

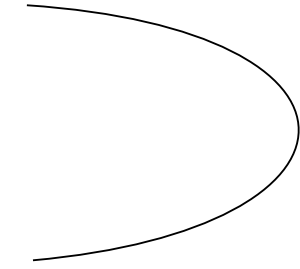

Notch blunting

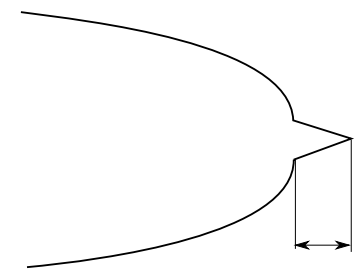

Crack nucleation

Figure 4.16: Scheme of crack advance initiation in normal tests. 


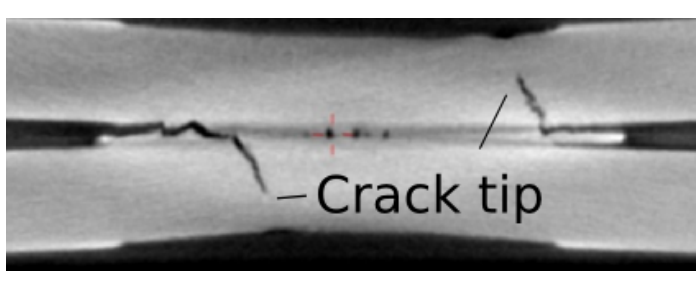

(a) 2D image.

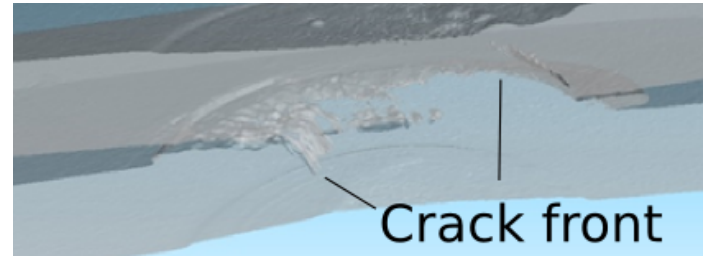

(b) 3D image.

Figure 4.17: Micro CT photo of cross sectioned weld of an interrupted normal test. A complex 3D crack propagation is observed.

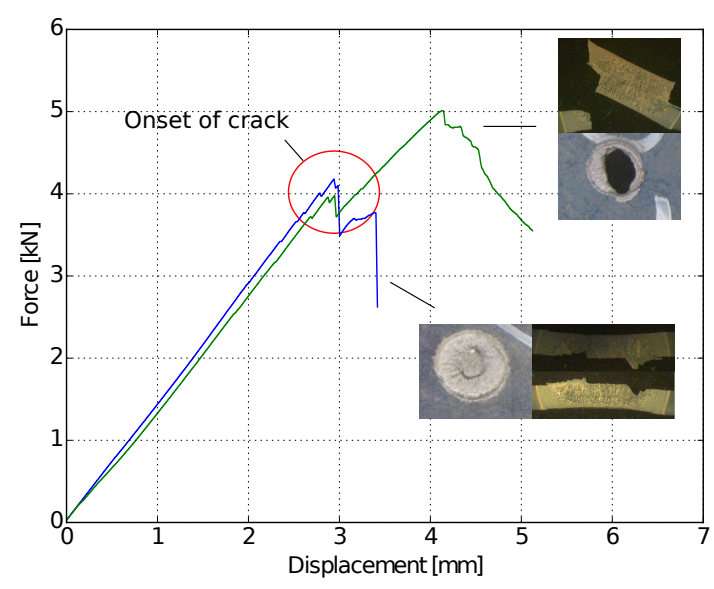

Figure 4.18: Differences in force-displacement curves of normal tests depending on crack front advance path. During the test of the blue curve, the crack crossed the nugget after the onset of the crack splitting the nugget in two parts and leading to lower forces. However, in the test of green curve, the crack progation was different on both sides of the nugget and could bear more up to the final collapse.

Independent of the fracture mechanism, the crack front propagation direction determines the final appearance of the failed spot weld and the failure mode following the clasificacion presented in Figure 2.9. (i) a fracture in the nugget boundary leads to partial or total dome fracture mode (PDF-TDF), and (ii) a fracture through the nugget leads to partial or full interface failure mode (PIF-FIF). For all cases, the crack propagation is triggered by strain/stress concentration around the notch and the only difference is the path followed by the crack front. In general, this direction is determined by two factors, the loading mode and the local material properties. In most cases, the crack front tends to follow the faying surface direction leading to FIF and PIF failure mode. However, when one of the sheets is much thinner than the other, this direction is very close to the nugget boundary. The nugget 
boundary has an extremely localized soft zone (see Figure 4.5 and [99]), and the crack propagation follows this path leading to $\mathrm{PDF}$ or TDF failure mode. Both types of crack propagation are presented in the failed cross-sectioned spot welds of Figure 4.19.
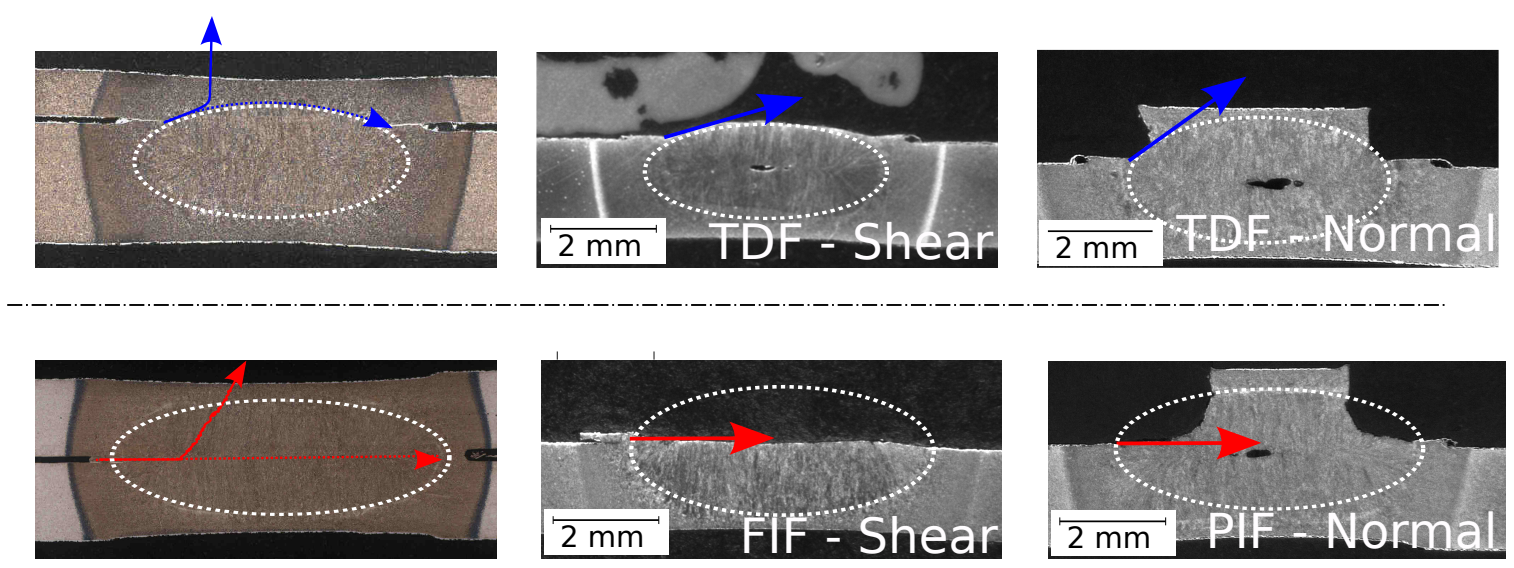

Figure 4.19: Cross sectioned spot welds of 22MnB5 joints. The first crack front propagation depends on the sheet thickness ratio. This leads to a different failed spot weld final appearance.

\begin{tabular}{lccc}
\hline Joint code & Shear & Mix-mode & Normal \\
\hline U08U08n & FIF - PDF & FIF-TDF-BPO & PIF-PDF \\
U08U15n & PDF & TDF-PDF & PDF \\
U08U20n & PDF -TDF & TDF-PDF & PDF \\
U15U15m & FIF & $* * *$ & FIF \\
U15U15n & FIF & $* * *$ & PIF-FIF \\
U15U15M & FIF & FIF & PIF-FIF \\
U15U20n & FIF & FIF & PIF-FIF \\
U20U20n & FIF & $* * *$ & FIF \\
\hline
\end{tabular}

Table 4.4: Spot weld failure modes for each thickness combination, weld diameter and loading mode of $22 \mathrm{MnB} 5$ joints.

The failure mode of all tests is presented in Table 4.4. It can be observed that most of the joints show a FIF failure mode, only U08U15n and U08U20n joints present PDF due to the high thickness ratio between the thicker and thinner sheet. It should be noticed that in the case of U08U08n the failure mode variability is higher, because the low dimensions make the imperfection of the weld get a higher importance during 
failure. Figure 4.20 shows multiple failed spot welds with a corresponding failure mode, mode II is observed in all shear tests and mode I fracture in the case of normal tests. Also, take note that weld solidification voids are observed in several cross sectioned welds. These voids may reduce the bearing capacity of the joints.

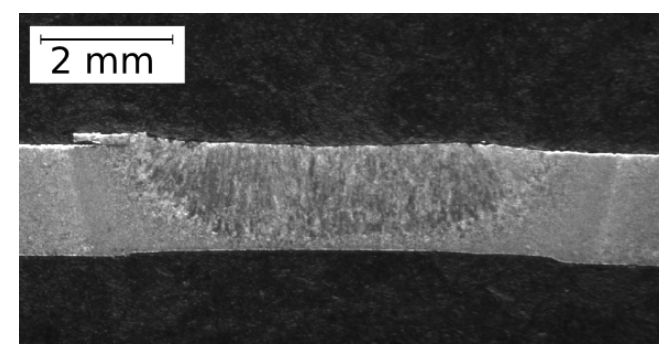

(a) FIF - Shear test.

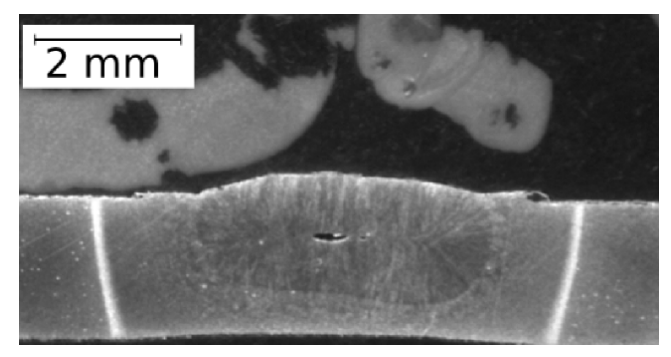

(c) TDF - Shear test.

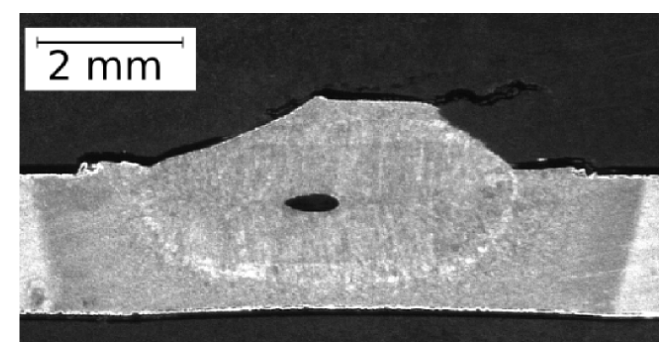

(e) PDF - Shear test.

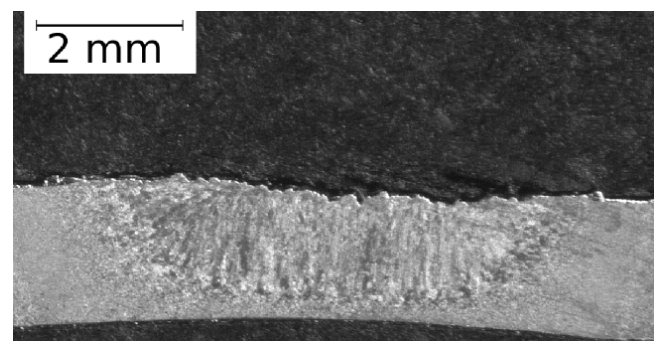

(b) FIF - Normal test.

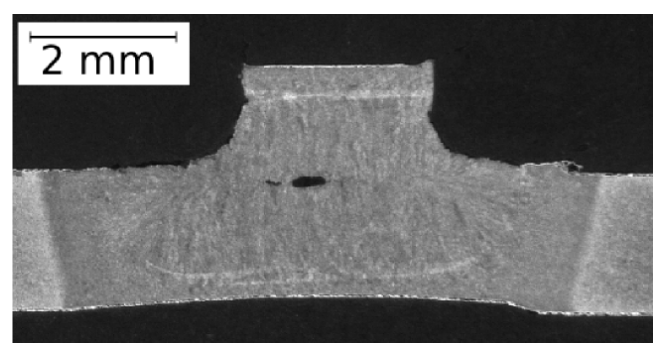

(d) PIF - Normal test.

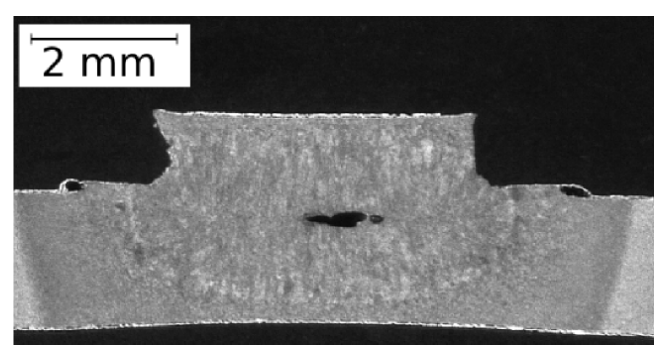

(f) PDF - Normal test.

Figure 4.20: Cross-sectioned failed spot welds of 22MnB5 similar joints and corresponding failure mode. The shear test spot weld can be seen on the left side and all of these have a failure due to mode II fracture with different crack front advance leading to multiple failure modes. On the right it can be observed the failed spot welds of the normal tests where ductile mode I failure and this can be observed for all cases. 
4.5. Model validation

\subsection{Model validation}

The validation of the spot weld FEM model using the experimental tests shown in the previous section is now presented. The features of the model related with the 22MnB5 joints are depicted in Section 4.5.1 and force-displacement curves obtained from the simulations are compared with the experimental results in Section 4.5.2. Finally, the obtained experimental and simulated maximum forces are compared in Section 4.5 .3 .

\subsubsection{Detailed model features of $22 \mathrm{MnB5}$ joints}

The numerical modeling features introduced in Chapter 3 are used to build up the spot weld model: detailed $0.1 \mathrm{~mm}$ mesh to describe the weld geometry, detailed description of the strain hardening plasticity model of the multiple weld zones and $J$-integral evaluated at the critical section and direction of the weld notch. The geometry and welding zones of the multiple tests presented in Section 4.1.1 are defined in the model, for all the multiple thickness combinations and the welding diameters. The nugget is defined as an oblate spheroid following the Eq. 3.5. The center is situated in the middle point between both sheets, i.e., in case of both sheets of the same thickness in the faying surface, and in the case of dissimilar thickness combinations at the side of the thicker sheet. The corresponding HAZ dimensions for each diameter and thickness' combinations are introduced using a numerical parametrization inspired by the hardening profiles presented in Section 4.2.3. The cylindrical hardness mapping is applied using the piecewise defined function:

$$
H V(r)= \begin{cases}H V^{N} & \text { if } r<r_{n} \\ H V^{H H A Z} & \text { if } r_{n}<r<r_{n}+\gamma_{0}=r_{0} \\ H V^{H H A Z}-\frac{H V^{H H A Z}-H V^{C H A Z}}{\gamma_{1}}\left(r-r_{0}\right) & \text { if } r_{0}<r<r_{0}+\gamma_{1}=r_{1} \\ H V^{C H A Z}+\frac{H V^{B M}-H V^{C H A Z}}{\gamma_{2}}\left(r-r_{1}\right) & \text { if } r_{1}<r<r_{1}+\gamma_{2}=r_{2} \\ H V^{B M} & \text { if } r>r_{2}\end{cases}
$$

where the different dimensions are defined in the HV scheme of Figure 4.21, $r$ is the $\mathrm{x}$-distance from the nugget central axis (see Figure 3.1). The $H V$ refers to Vickers' 
hardness values, and superindex $H V^{N}, H V^{H H A Z}, H V^{C H A Z}$, and $H V^{B M}$ refer to the nugget, hard HAZ, critical HAZ and BM. The values of the multiple parameters are extracted from the hardness measurements of Section 4.2.3. $r_{n}$ represents the nugget radio. The dimensions of the zones are set to $\gamma_{0}=1.25 \mathrm{~mm}, \gamma_{1}=0.25 \mathrm{~mm}$ and $\gamma_{0}=1.50 \mathrm{~mm}$. Fully hardened 22MnB5 mechanical properties are applied for nugget, HHAZ and BM following the conclusions extracted in Section 4.3.2. Therefore, the hardness values are $H V^{N}=H V^{H H A Z}=H V^{B M}=500$ and $H V^{C H A Z}=325$. Notice that the focus of this work is not the detailed characterization of these zones and more detailed hardness profiles are explained in [18].

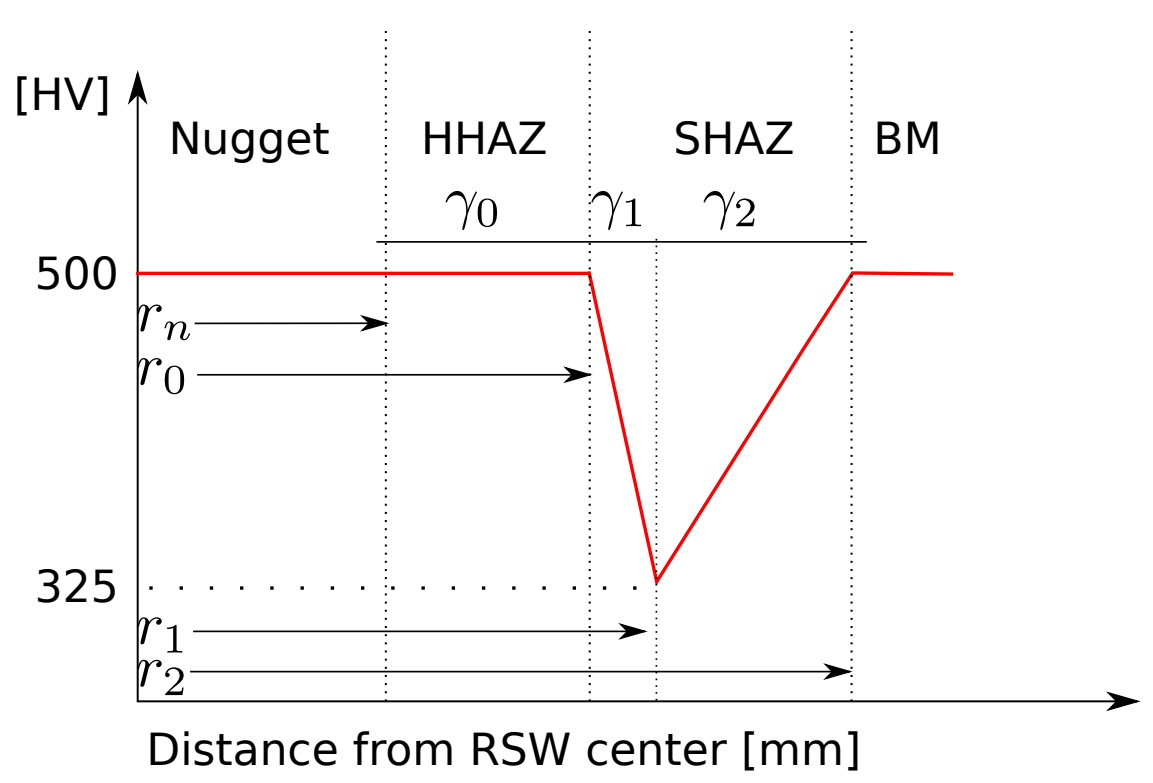

Figure 4.21: Simplified hardness profiles used for the hardness-based constitutive model of the welding zones. It is based on the experimental hardness profile presented in Figure 4.5.

Also, the extremely confined soft zone that has been reported in the fusion zone boundary represented in Figure 4.5 has not been introduced in the hardness profile used for the weld zones modeling presented in Figure 4.21. The mechanical properties drop of this confined zone cannot be characterized due to their tiny dimensions and therefore cannot be modeled in the FEM model. However it may lead to a small reduction of the experimental maximum forces in joints with extremely different thickness combinations. 


\subsubsection{Force-displacement curves validation}

Figure 4.22(a) shows the simulated force-displacement curves of shear tests, for joints of multiple thickness combinations and different spot weld diameters. The curves are plotted to the maximum forces extracted from Figure 4.22(b). In this figure, the evolution of $J$-integral with the applied force has been plotted. The maximum forces of the force-displacement curves are extracted from the intersection between these curves and critical $J$-integral for mode II fracture $J_{I I C}$. The $J_{I I C}$ value is set to $56 \mathrm{~kJ} \mathrm{~m}^{-2}$ as introduced in previous literature for a different steel (TRIP780) with the same nugget martensitic hardness $(500 \mathrm{HV})$ and therefore a similar fracture toughness [10]. A strong sensitivity to the spot weld diameter for constant thickness' combination can be observed for both types of curves (see combinations U15U15m and $\mathrm{U} 15 \mathrm{U} 15 \mathrm{M})$. In fact, all curves are more sensitive to the weld diameter than to the thickness combination: the curves of U08U15n are similar to those of U15U15m and the curves of U15U20n are similar to those of U15U15M because the diameters of both pairs are very close (see Table 4.2). On the other hand, it can be observed that the $J$-integral evolution curves are steep after some applied force value. For this reason, the maximum force is not sensitive to the critical $J$-integral value.

The experimental results of two combinations are also plotted in the Figure 4.22(a), considering the DIC measurements. A good agreement can be observed between the experimental and simulated curves up to fracture.

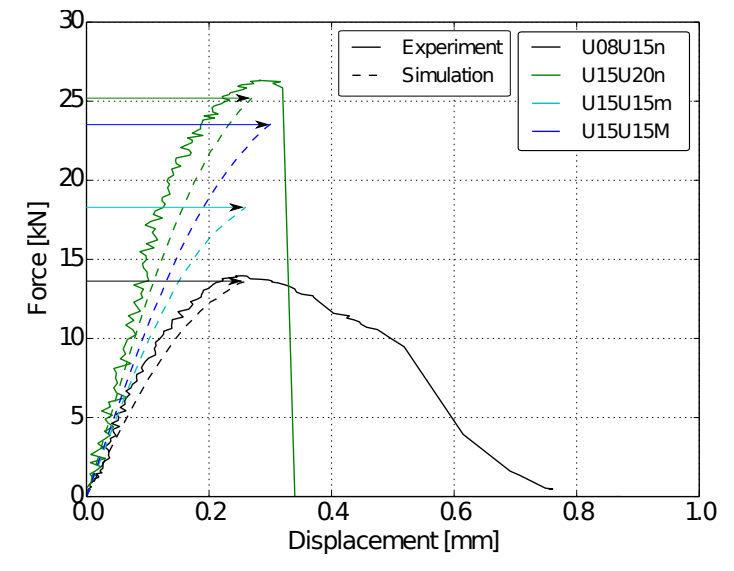

(a) Force-displacement curves.

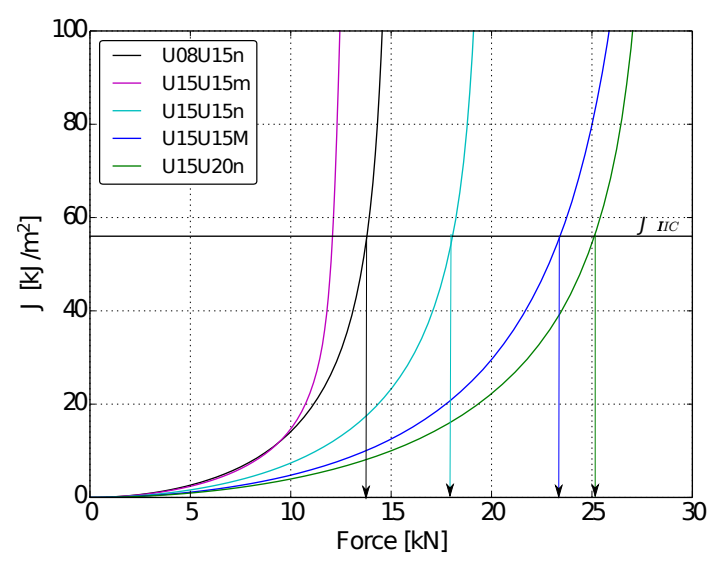

(b) $J$-integral evolution.

Figure 4.22: Simulation results of shear tests of 22MnB5 joints with the same thickness combination and multiple diameters. 
The simulated and experimental force-displacement curves of normal tests of two different thickness combinations are presented in Figure 4.23(a). In this case the agreement between experiments and simulations is lower in terms of displacements due to unreliable boundary conditions. It can be explained by considering the high sensitivity of the displacements to the boundary conditions during this loading test as presented in Figure 4.24. The $J$-integral evolution is plotted in Figure 4.23(b) for multiple thickness combinations and weld diameters and a higher dependency on the thickness combination than in shear tests is observed. As opposed to shear tests, neither the force-displacement curve nor the $J$-integral evolution curve have high sensitivity to the spot weld diameter (see Figure 4.23(b)), as already observed in the experimental tests. The critical forces are obtained with the intersection of $J$-integral curve and critical $J$-integral. In the same way as in the shear case, this critical value is obtained from previous literature, where the fracture of spot welds in a TRIP780 steel was analyzed in normal loading conditions and $J_{I C}$ was set to $22.5 \mathrm{~kJ} \mathrm{~m}^{-2}$ [17]. It must pointed out that $J$-integral-force curves are not as steep as in the shear case. Consequently, sensitivity of the maximum forces to the critical $J$-integral value in this mode I fracture $\left(J_{I C}\right)$ is higher.

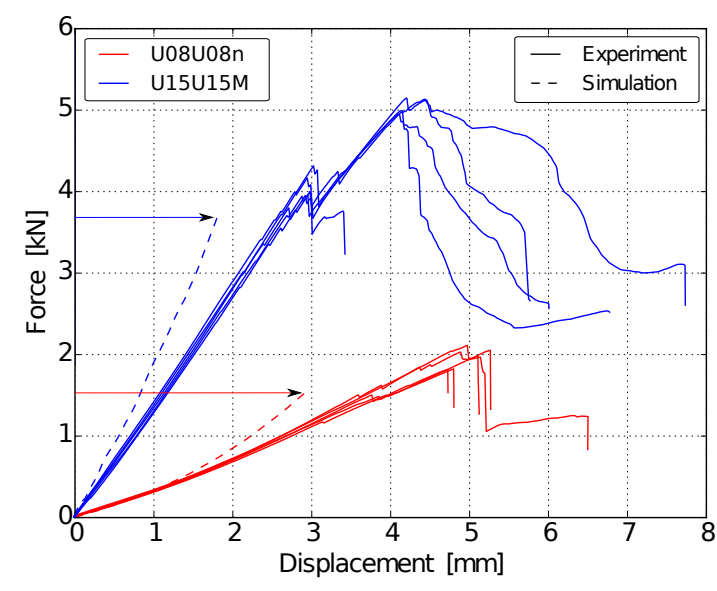

(a) Force-displacement curve.

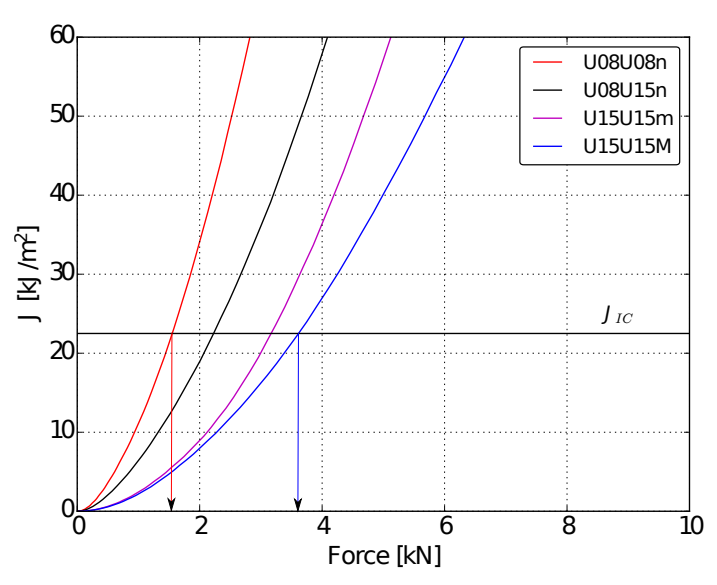

(b) $J$-integral evolution.

Figure 4.23: Simulation results for 22MnB5 joints of normal tests with multiple spot weld diameters.

Force-displacement curves of simulation and experimental mix-mode tests are shown in Figure 4.25(a), The simulations must introduce not only the applied displacements in the vertical direction as boundary condition but also the lateral dis- 


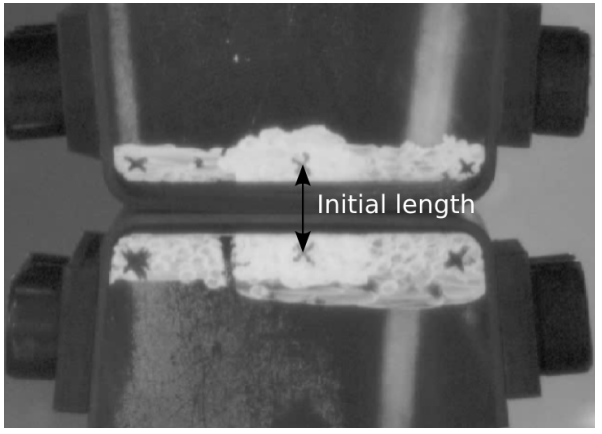

(a) Initial state.

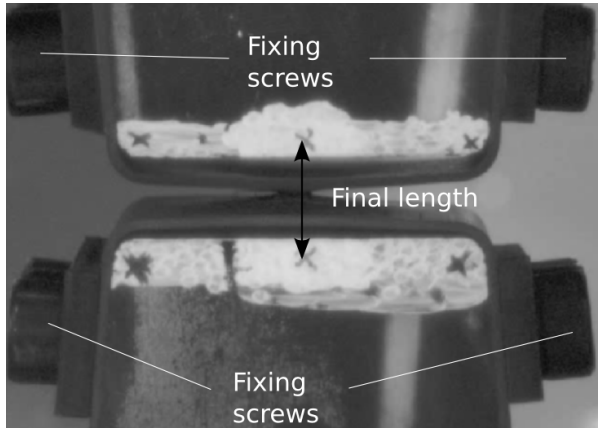

(b) Final state.

Figure 4.24: Front view of normal test. Small differences in the fix conditions would lead to large differences in the displacements due to the variations in the bending of the material surrounding the weld during loading.

placements experimentally measured. A good agreement can be seen between experimental and simulated results. The differences in the displacements are a product of the unriable boundary condition related to normal applied displacements. The $J$-integral evolution is plotted in Figure 4.25(b) for different thickness combinations. These curves are more similar to those observed in shear tests, i.e. they are steep after some point and they are strongly sensitive to spot weld diameter. Mode II shear fracture has been observed experimentally, consequently $J_{I I C}=56 \mathrm{~kJ} \mathrm{~m}^{-2}$ is used as critical value for the prediction of maximum forces.

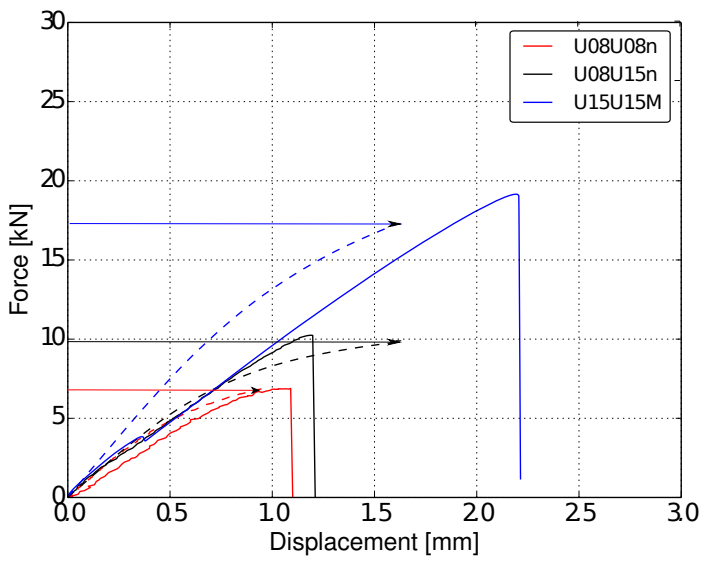

(a) Mix-mode tests.

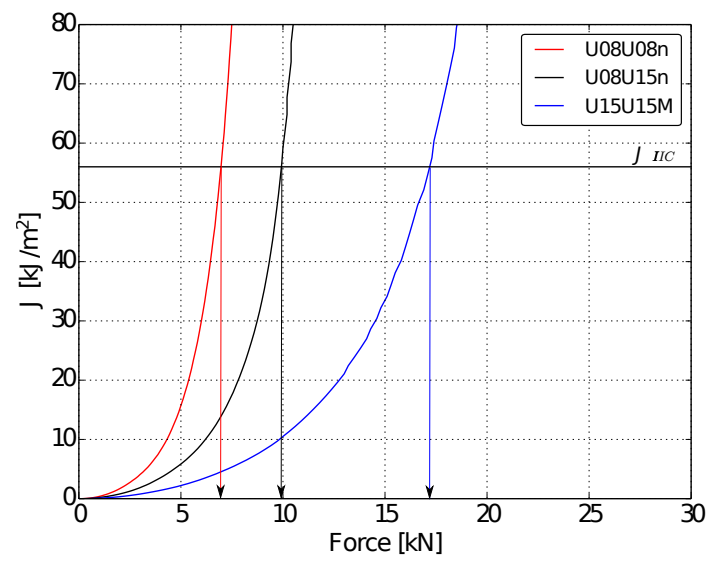

(b) Shear tests.

Figure 4.25: Simulation results for 22MnB5 joints of mix-mode tests with multiple spot weld sheet thickness combinations. 


\subsubsection{Maximum forces validation}

The maximum forces resulting from all the experimental tests and from the FEM model followed the procedure presented in the previous section are presented in Figure 4.26. The results of the shear tests are presented in Figure 4.26(a), A good agreement can be observed between the experimental and simulated results. The small discrepancies, however, result mainly from an error in spot weld diameter measurements and the variation of the weld sizes even for the same thickness combination using the same set of welding parameters. In Figure 4.27 this strong sensitivity of the maximum forces of the shear test to the weld size and its measurement errors can be observed. The higher differences between experimental and simulated results can be seen in the extreme combinations, such as in U08U08n and U20U20n. In the case of U08U08n this underestimation is caused by the irregularities in the spot weld geometry that have a greater importance with small sheet and spot welds dimensions. In the case of U20U20n the lower experimental forces are consequence of the voids observed in the weld nugget (see Figure 4.4).

Normal test results are presented in Figure 4.26(b). The forces of crack nucleatio, i.e. the onset of crack, detected in the force-displacement curves were used as the experimental critical forces as demonstrated in Figure 4.18. The simulated results follow the trend of the experimental forces with a small underestimation for all cases. Notice that for normal tests the higher scatter in the maximum forces is observed, it results from the irregularities in the weld notch that have an influence on the first crack nucleation during loading. The underestimation is probably attributed to the low value of critical $J$-integral for mode I $\left(J_{I C}=22.5 \mathrm{~kJ} \mathrm{~m}^{-2}\right)$ used in this thesis. This value can be fitted more precisely to nugget material using independent experimental tests. However, the results will not improve significantly and this characterization is beyond the scope of this thesis.

Finally, mix-mode test results are presented in Figure 4.26(c). A good agreement can be observed between experimental and simulated forces. Notice that in these tests, the shear component of the applied displacements determines the mode II fracture, therefore the errors in the nugget measurements introduce the errors in the maximum forces calculations in the same as the shear tests.

The same simulated results for all tests types are plotted in Figure $4.26(\mathrm{~d})$ using a common scale. It can be seen that shear test forces are much higher than in normal tests. As presented previously, the results of the mix-mode tests are closer to the 


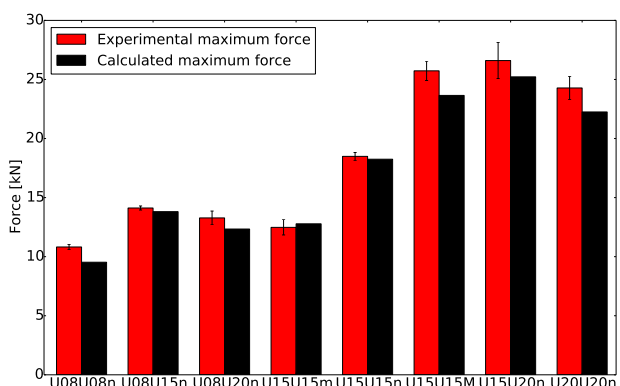

(a) Shear tests.

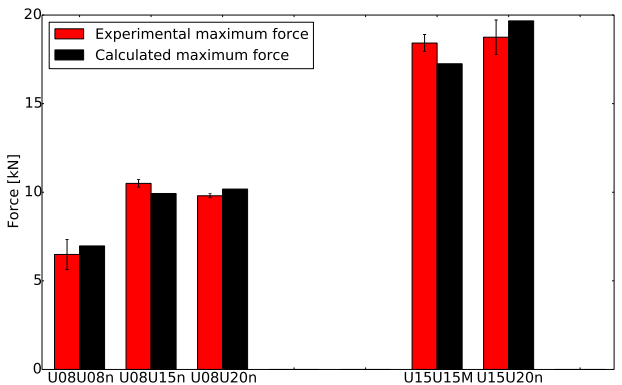

(c) Mix-mode tests.

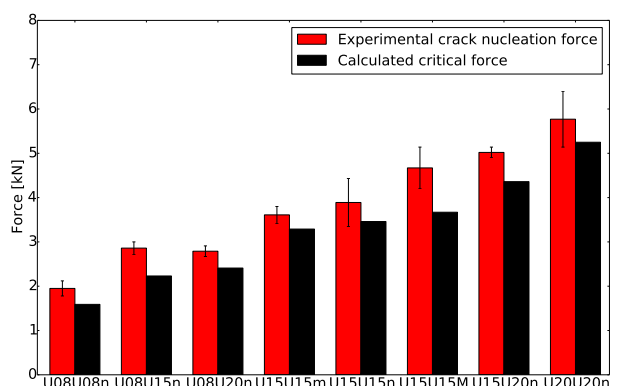

(b) Normal tests.

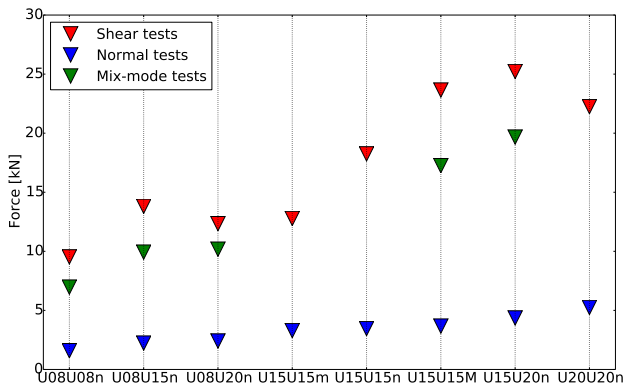

(d) Simulated results of all cases.

Figure 4.26: A comparison of calculated and experimental critical forces of 22MnB5 joints. The experimental forces are plotted with error bars representing the standard deviation considering the multiple tests used for each combination.

shear tests, due to the higher relevance of the shear component. Another result from these simulated tests in that the increase of both sheets thicknesses sum corresponds with an increase of the maximum forces. However, the reason is different depending on each loading case. Figure 4.27 plots the results of both normal and shear tests of sheets combinations with the same thickness and compares the maximum forces depending on the weld diameter. In the case of shear tests, the maximum forces variation results from the weld diameter variation that is related not only to the thickness combination but also to the welding parameters. However, for normal tests the sensitivity to the weld diameter is very low. The influence of the thickness combination observed for these tests does not result from the weld diameter but rather the sheets' thicknesses due to different material bending around the weld and the different crack front opening angle. 


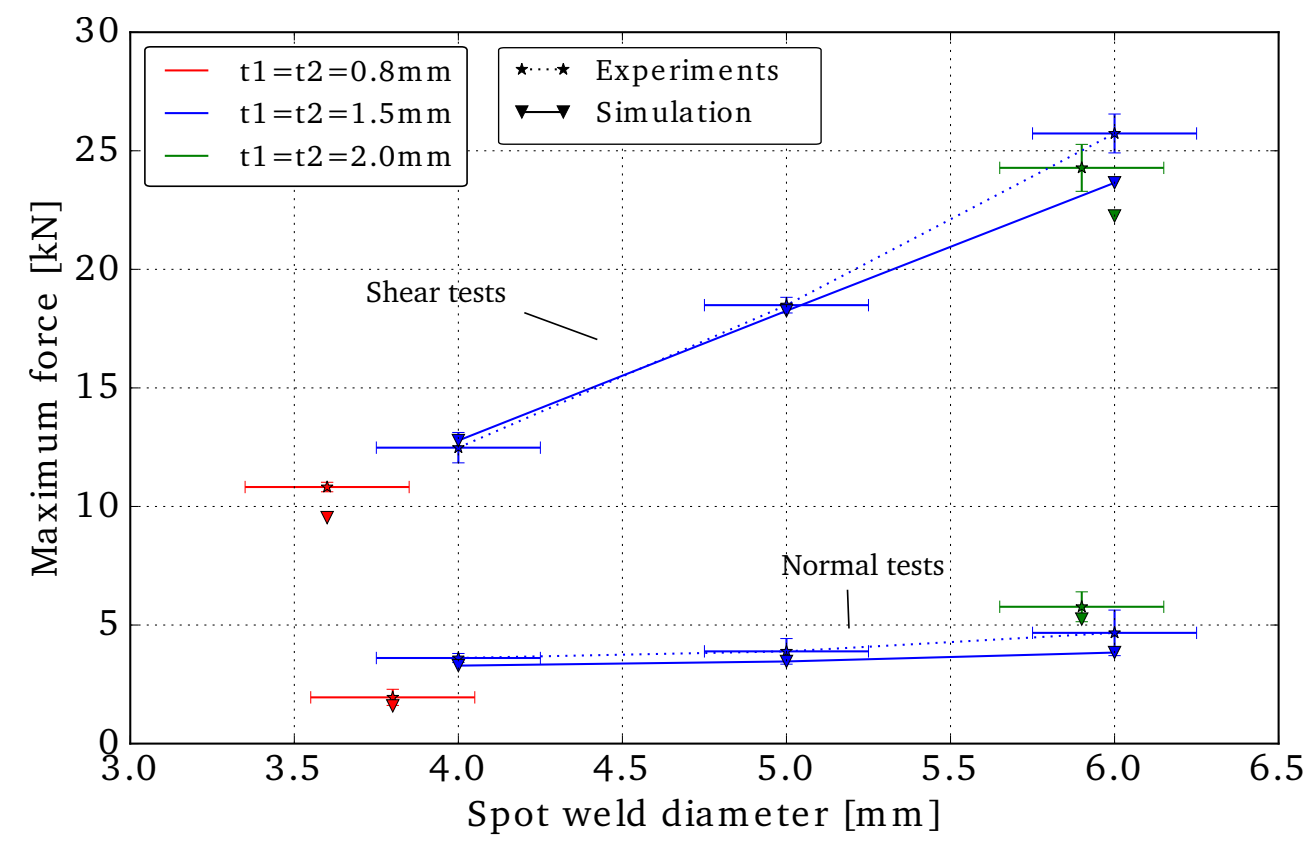

Figure 4.27: Maximum forces represented as a function of the weld diameter in the case of $22 \mathrm{MnB} 5$ joints. The experimental results are plotted with error bars for the forces representing the standard deviation and error bars of welding diameters considering the measurement errors of $0.2 \pm \mathrm{mm}$. The colors indicate the different thickness combinations. 


\section{Chapter 5}

\section{Application in resistance spot welds of a cold formed steel}

The spot weld model presented in the previous chapters is further explained in this chapter for joints of a different steel grade within the AHSS family, specially the dual phase steel DP 980. This is a cold formed steel, with a dual phase martensitic-ferritic microstructure extensively used in the automotive industry. More details of this steel can be found in Section 2.2. Under the spot weld failure modeling point of view, the different microstructure of this steel introduces a new material properties distribution around the weld leading to different fracture response.

The same procedure followed in Chapter 4 for the 22MnB5 joints is followed here. The test matrix used for the calibration and the validation of the FEM model is presented in Section 5.1. The geometry, dimensions and spot weld zones are analyzed in Section 5.2. Following this analysis, the nugget material properties of this type of joints must be characterized in order to complete the FEM model. This characterization is demonstrated in Section 5.3 using micro-tensile tests and an inverse FEM optimization scheme. The experimental loading and fracture results of the welded joints are presented in Section 5.4. Finally, the features of the spot weld model are presented in Section 5.5 and the simulated results are compared with experimental results with validation purposes. 


\subsection{Experimental validation campaign}

The design of the test matrix follows similar criteria to those followed for the 22MnB5 joints in Section 4.1. In regards to the multiple thickness combinations, it is considered that the main geometrical features of spot welds have been already analyzed for similar 22MnB5 joints in the previous chapter of this thesis. Consequently, only one thickness combination is characterized in this chapter. The combination of two of $1.5 \mathrm{~mm}$ sheets has been selected for this purpose. The weld size is considered an important factor to analyze. For this reason, specimens are welded using three different sets of welding parameters to obtain the maximum $\left(d_{M}\right)$, minimum $\left(d_{m}\right)$ and nominal $\left(d_{n}\right)$ weld diameters, these parameters are presented in Table 5.2 and the corresponding diameters obtained are given in Table 5.1. Notice that the welding process of these joints have only one current pulse. All specimens are loaded using the shear and normal tests following the scheme of Figure 3.4. The specimens with nominal diameter (D15D15n) are loaded using the mix-mode tests as well.

\begin{tabular}{l|cc|cc|cc}
\hline \multicolumn{1}{c}{ Joint } & \multicolumn{2}{c}{ Sheet 1 } & \multicolumn{2}{c}{ Sheet 2 } & \multicolumn{2}{c}{ Diameter (mm) } \\
Code & Mat & $t_{1}(\mathrm{~mm})$ & Mat & $t_{2}(\mathrm{~mm})$ & Target & Obtained \\
\hline D15D15m & DP 980 & 1.5 & DP 980 & 1.5 & $d_{m}<5.5$ & $5.10 \pm 0.2$ \\
D15D15n & DP 980 & 1.5 & DP 980 & 1.5 & $d_{n}=5.5$ & $6.10 \pm 0.2$ \\
D15D15M & DP 980 & 1.5 & DP 980 & 1.5 & $d_{M}>5.5$ & $7.20 \pm 0.2$ \\
\hline
\end{tabular}

Table 5.1: Sheet and material thickness combinations and corresponding target and obtained nugget diameter in similar DP 980 joints.

\begin{tabular}{l|c|cc|ccccc}
\hline Joint & \multicolumn{2}{c}{ Force $(\mathrm{kN})$} & \multicolumn{2}{c}{ Intensities $(\mathrm{kA})$} & \multicolumn{5}{c}{ Time $(\mathrm{ms})$} \\
Code & $\mathrm{F}$ & $I_{1}$ & $I_{2}$ & $t_{s q}$ & $t_{s 1}$ & $t_{f 1}$ & $t_{s 2}$ & $t_{f 2}$ \\
\hline D15D15n & 3.50 & 0.00 & 5.50 & 500 & 000 & 00 & 400 & 200 \\
D15D15m & 3.50 & 0.00 & 6.60 & 500 & 000 & 00 & 400 & 200 \\
D15D15M & 3.50 & 0.00 & 7.80 & 500 & 000 & 00 & 400 & 200 \\
\hline
\end{tabular}

Table 5.2: Welding parameters for all thickness combinations and target diameters in DP 980 joints. All weldings follow the scheme of Figure 4.1, without the first welding pulse. 


\subsection{Geometry and local material properties distribution of the spot welds}

In order to characterize the material properties changes and the geometry resulting from the welding process, the joints are analyzed following the same procedure already presented in Section 4.2. The welds are cross-sectioned and a Vickers hardness is measured with a line crossing the nugget from one sheet to the other.

Slight differences can be observed in the spot weld geometry of these DP 980 joints (presented in Figure 5.1) when comparing with 22MnB5 joints (represented in Figure 4.2). However, the DP 980 spot welds present a sharper notch as observed in Figure 5.3(f) due to the fact that Zn coating of this steel is thinner and does not introduce the irregularities in the weld limits that are observed in the $22 \mathrm{MnB} 5$ joints (see Figure 4.3).

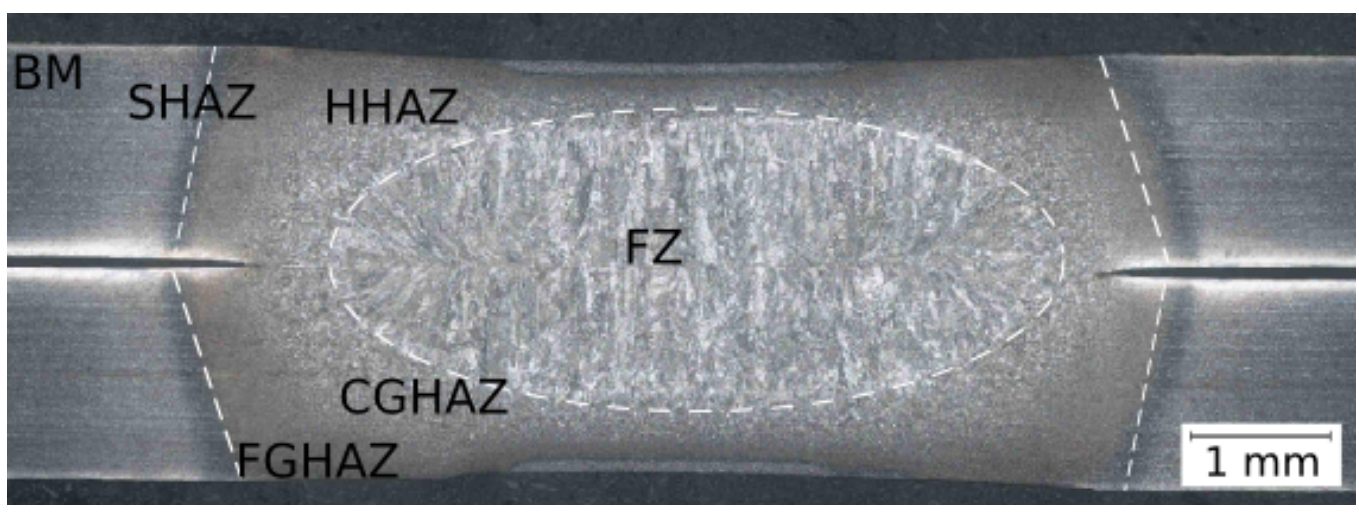

Figure 5.1: Cross sectioned spot weld of DP 980 similar joints.

Figure 5.2 shows the hardness measurements and a scheme of the hardness profile of a similar DP 980 joint. A hardness of 340-350 HV can be observed in the BM. Continuing towards the weld center, a decrease of hardness values until $300 \mathrm{HV}$ is observed in the SHAZ. The hardness drop between BM and SHAZ hardness is much lower than in 22MnB5 joints because the BM hardness of the latter ones is much higher. Finally, around $400 \mathrm{HV}$ are measured in the nugget and in the HHAZ. In the boundary of the HHAZ higher values of hardness (almost $450 \mathrm{HV}$ ) are presented due to the finer grain size in this zone. The lower hardness values of the fusion zone when comparing with $22 \mathrm{MnB} 5$ joints nugget can be explained taking into account that the martensitic hardness is strongly dependent on the steel carbon content [27]. 
The DP 980 steel has a lower carbon content than 22MnB5 (see Table 2.2 and Table 2.2), therefore, its martensitic hardness is lower. The hardness profile can be bettter explained when observing the micrographs of the multiple zones presented in Figure 5.3 .

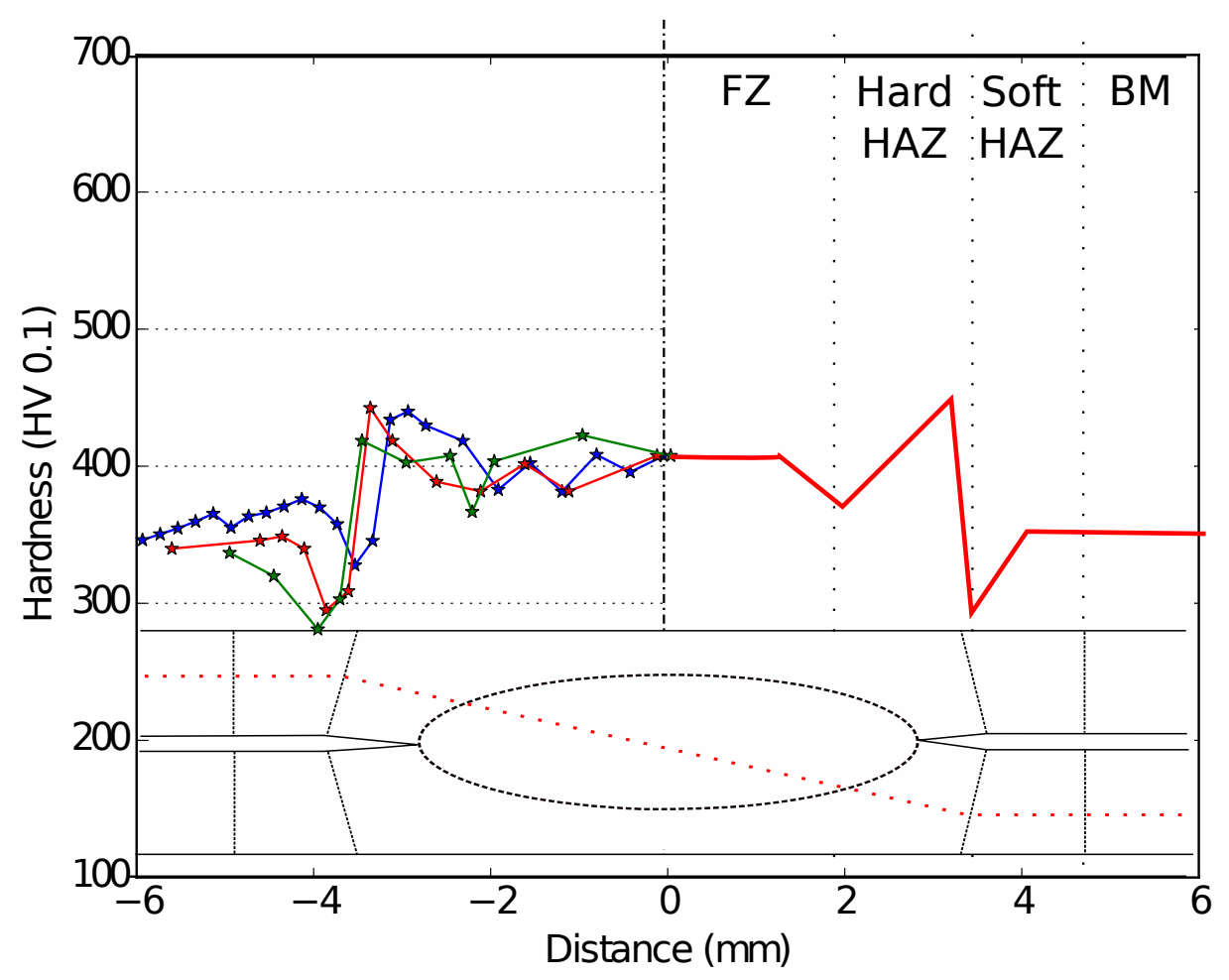

Figure 5.2: Vickers hardness profile of a similar joint of DP980 steel. Left side: Hardness measurements of three different specimens welded with the same conditions. Right side: Scheme of the hardness profile with the main weld zones.

Summarizing, for this type of joint, two regions can be considered from the modeling point of view of material properties. Both FZ and HHAZ with pure martensite microstructure, and on the other side the BM with dual phase microstructure. Micro tensile tests are performed in Section 5.3.1 to characterized these zones. The softening of the SHAZ is considered negligible in these joints due to the low hardness drop between FZ and SHAZ, 

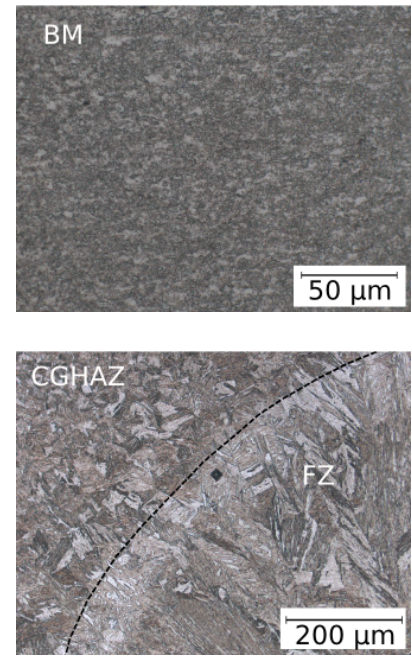
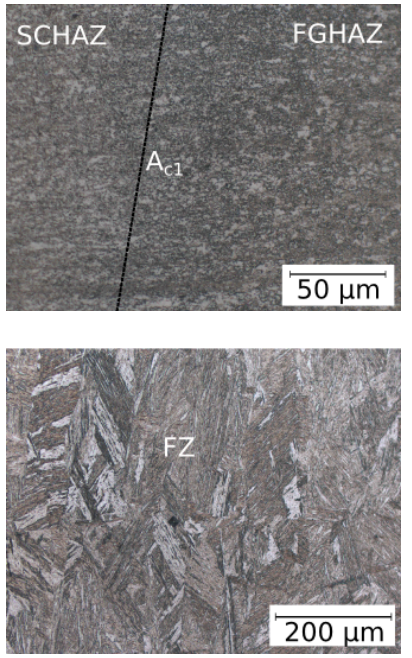
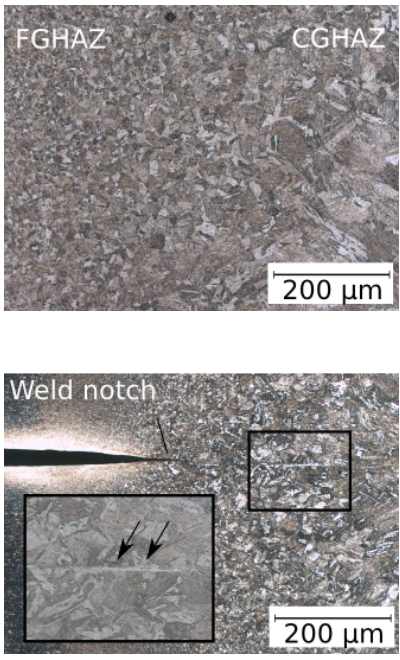

Figure 5.3: Optical micrographs of multiple spot weld zones of a DP 980 joint. From top left to bottom right: It can be seen that BM consists of a ferritic matrix with dispersed martensite, with a great volume fraction of the latter and small presence of ferrite. Microstructure show a slightly banded structure (banding aligned with the rolling direction). The material of the so-called SHAZ has reached temperatures bellow $\mathrm{Ac}_{1}$ leading to tempering of the martensite present in the $\mathrm{BM}$ and lower values of hardness. As well as in the 22MnB5 joints, the FGHAZ and CGHAZ have suffered complete reaustenization after being above $\mathrm{Ac}_{3}$. This austenite is transformed again into martensite after the rapid cooling. The short periods of time above this temperature produce the ultra fine grains of the FGHAZ leading to high hardness values. Finally, predominantly needle-like martensitic microstructure is present in the FZ, characterized by the columnar grains growing towards the center of the nugget resulting from the melting process and rapid cooling.

\subsection{Characterization of nugget material properties}

As presented in the previous section, the strain hardening behavior of BM and nugget DP 980 joints must be characterized in order to complete the spot weld constitutive model. The characterization of the $\mathrm{BM}$ can be performed following the classical approaches using large coupons of raw material. A previous calibration of this materials hardening curves is used in this thesis. However, due to the small dimensions, the parameters of the nugget material's plasticity model must be calculated using a different approach. In this thesis, the fracture criterion is not based on the plastic strains, consequently a calibration of these curves extremely accurate is not needed, 
therefore, only a first order approximation is applied. Plasticity model parameters, such as the strain hardening curves of the fusion zone, are obtained from an inverse FEM optimization procedure presented by Eller et al. [102]. In which the experimental results of the micro tensile tests are compared with FEM simulations and the parameters of the strain hardening curve are modified iteratively until reaching the desired values.

\subsubsection{Mechanical tests}

The specimens are loaded with the Zwick machine using the same procedure presented in Section 4.3. The obtained force-displacement curves are shown in Figures 5.4 . Unlike in the case of $22 \mathrm{MnB} 5$, some differences are observed between welded and unwelded specimens' curves. Slightly higher forces are reached in those specimens with weld material in the gauge zone, however the fracture displacements are lower in the welded specimens. The shape of the curves after the elastic part is not the same for both specimen types, and they cross each other in the UT test case. These phenomena can be explained by taking into account that both $\mathrm{FZ}$ and BM have different microstructure as introduced in the previous section. The ferrite phases of DP $980 \mathrm{BM}$ gives it this more ductile behavior. However, the nugget material is completely martensitic, and it is therefore more brittle. Much lower maximum forces are reached in both cases when comparing with $22 \mathrm{MnB} 5$ tests presented in Figures 4.10 .

For these reasons, plasticity of DP $980 \mathrm{BM}$ and the nugget material of the DP 980 welds is different. Consequently, the hardening curve of nugget material must be calibrated. The classical transformation of the UT force-displacement curves into true stress-strain plastic curve cannot be used in this case due to two reasons. It is only valid up to the point of maximum uniform elongation, which is the strain at which diffuse necking begins. Furthermore, the strain field is not homogeneous in the gauge zone, therefore the hypothesis for calculating the strain-stress curve using these specimens are not met. Consequently, a plasticity model is calibrated here using an inverse FEM optimization scheme. 


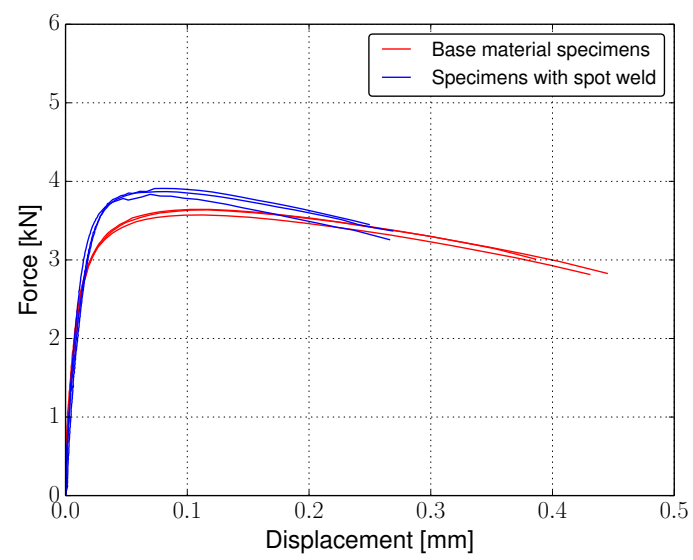

(a) PST tests.

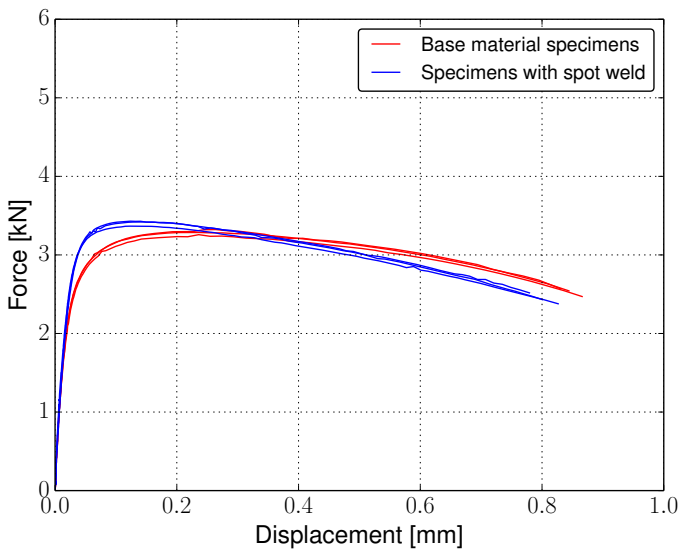

(b) UT tests.

Figure 5.4: Reduced tensile DP 980 tests with and without spot weld microstucture in the gauge zone.

\subsubsection{Plasticity model calibration}

The main focus of this section is on calibration of the strain hardening behavior of the DP 980 joints nugget material. An inverse FEM optimization scheme is used in order to obtain the strain hardening curves parameters. It uses the experimental force-displacement curves of the $\mathrm{UT}$ and $\mathrm{PST}$ tests presented in the previous section, and compare them with FEM simulations where the parameters of the plasticity model are changed iteratively to minimize a cost function. The simulation model assumptions are presented in this section, then a cost function is detailed and finally the obtained hardening curves are presented.

The simulation model represents the $\mathrm{UT}$ and $\mathrm{PST}$ specimens geometry using a 3D mesh of $0.1 \mathrm{~mm}$ following scheme of Figure 5.5. It is calculated using VPS and based in the same constitutive model used for the spot weld model that was presented in Section 3.2. A simple von Mises yield criterion is used because a more complex yield criterion is not necessary and would increase the computational costs. A previously obtained strain hardening curve is applied to the $\mathrm{BM}$ and SHAZ. The parameters of the strain hardening law of the FZ are fitted with this procedure. A Swift-Voce hardening law is the most suited to fit the curve for small and large strains. This law is the combination of power Swift law [103] and the Voce law [104]. The relation between yield stress $\left.\sigma_{S V}\right)$ and effective plastic strain $\left(\overline{\varepsilon_{p}}\right)$ is given by: 


$$
\sigma_{S V}=\alpha *\left(K\left(\varepsilon_{0}+\bar{\varepsilon}_{p}\right)^{n}\right)+(1-\alpha)\left(\sigma_{0}+Q_{1}\left(1-\exp \left(-C_{1} * \bar{\varepsilon}_{p}\right)\right)\right)
$$

where $K, \varepsilon_{0}$ and $n$ are the parameters of the Swift law, $\sigma_{0}, Q_{1}$ and $C_{1}$ are the parameters of the Voce law following [105], and $\alpha$ is a mixing parameter for the combination of both laws.

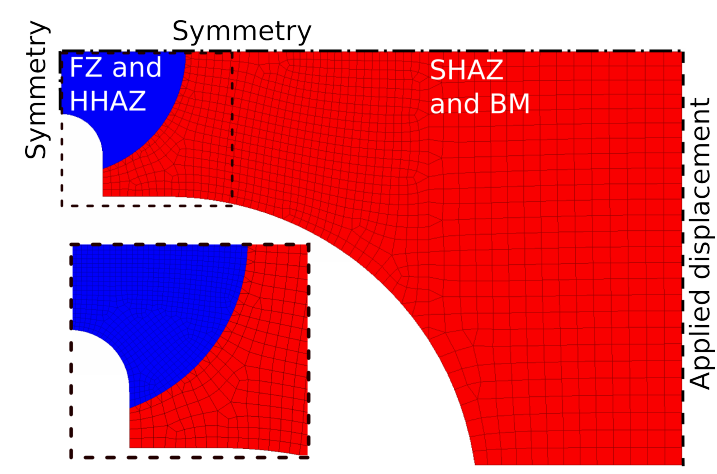

(a) PST specimen.

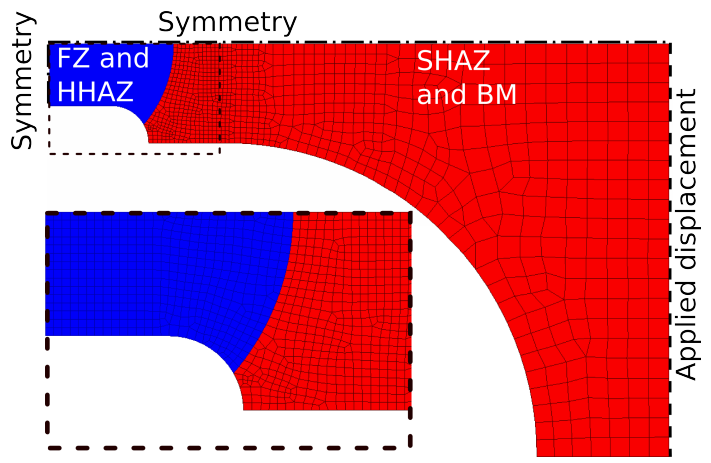

(b) UT specimen.

Figure 5.5: FEM model of the specimens used for the plasticity model calibration of the nugget material. The mesh is divided in two domains with corresponding hardening curves, on one side the BM and SHAZ, and on the other side the FZ along with the HHAZ. The FZ is defined with a cylindrical shape with a radius of $3 \mathrm{~mm}$ from the center of the specimen following the dimensions extracted from the hardness profile of Figure 5.2. Double symmetry is applied to model the experimental conditions and to avoid high computational costs.

To identify the model parameters of Eq. 5.1, the results of the simulations are compared with the experimental results using a cost function. Similar to the approach used by Eller [18], this cost function is defined by a weighted least-square deviation between simulated and experimental force-displacement curves:

$$
Y(\boldsymbol{X})=\beta Y_{U T}(\boldsymbol{X})+(1-\beta) Y_{P S T}(\boldsymbol{X})
$$

in which $\beta$ is a weighting factor, $\boldsymbol{X}=\left(K\left|\varepsilon_{0}\right| n\left|\sigma_{0}\right| Q_{1} \mid C_{1} \alpha\right)$ is the Swift-Voce hardening parameters vector, and $Y$ is defined as:

$$
Y_{U T}(\boldsymbol{X})=\frac{1}{n_{F}} \sum_{i=1}^{N_{F}}\left(\frac{F_{s i m}\left(d_{i}, \boldsymbol{X}\right)-F_{\exp }\left(d_{i}\right)}{\frac{1}{n_{F}} \sum_{j=1}^{n_{F}} F_{\exp }\left(d_{j}\right)}\right)^{2}
$$

where $n_{F}$ is the number of sample points of the force-displacement curve and $F_{\text {exp }}\left(d_{i}\right)$ is the force at sample displacement $d_{i}$. The cost function is defined likewise for the 
plastic strain tension tests, $Y_{P S T}$. The starting values of the parameter optimization are extracted from a previous work [105].

The strain hardening curves obtained with the inverse FEM optimization scheme are presented in Figures 5.6. They are compared with DP 980 base material curves. For the small strains, presented in Figure 5.6(a), both curves have a similar shape, with higher stresses obtained in the case of the martensitic fusion zone material. However, when both curves are analyzed up to large strains (see Figure 5.6(b)), it can be observed that they cross, the original dual phase base material reaches higher forces at higher strains. The accuracy of the calibration is not important, due to the fact that the material just reach a plastic strain in an extremely confined region close to the notch tip when the joining specimens are loaded.

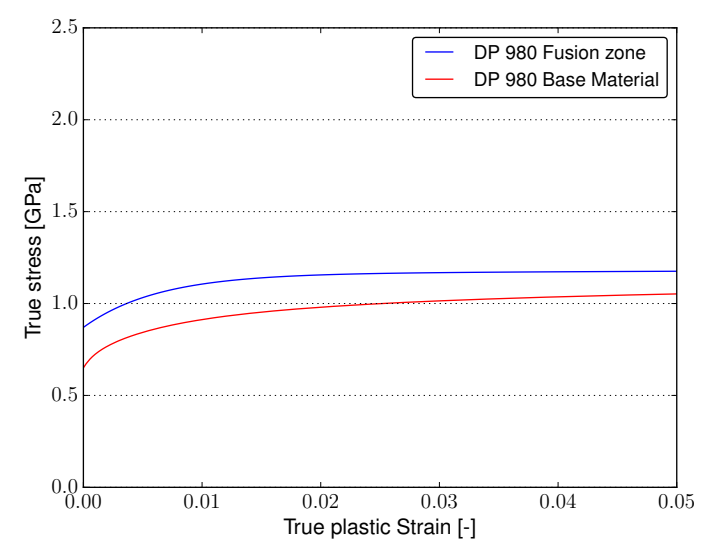

(a) Small strains.

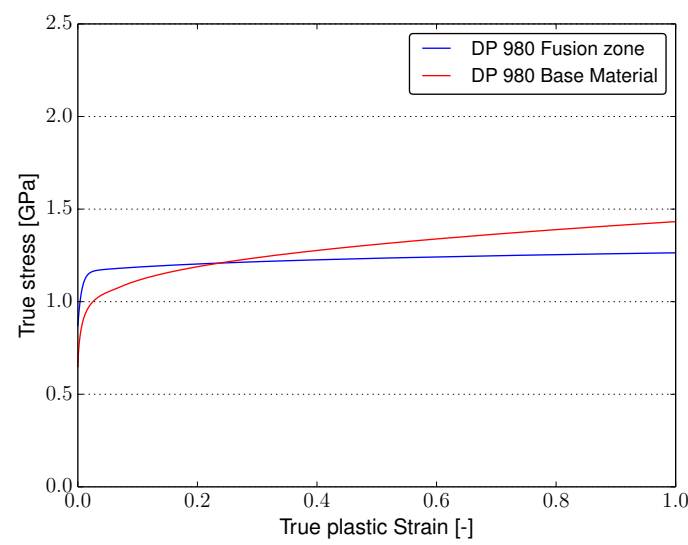

(b) Large strains.

Figure 5.6: Hardening curves obtained from the force-displacement curves using the inverse FEM optimization scheme.

\subsection{Experimental loading tests of the joining specimens}

The results of the experimental loading tests introduced in Section 5.1 are presented in this section. The followed welding and loading methodology is the one introduced for similar 22MnB5 joints in Section 4.4.1, i.e. the U-profile joining specimens are welded and loaded at three different angles up to fracture. 


\subsubsection{Force-displacement curves}

Figure $5.7(\mathrm{a})$ shows the shear test force-displacement curves of spot welds with three different diameters. Strong sensitivity to the weld diameter can be seen in these tests as already reported in the previous chapter for 22MnB5 similar joints (see Figure 4.11(b) . However, in this case the curves are not as brittle as the similar 22MnB5 joints, the force drop is not vertical after maximum force due to a stable crack propagation after crack nucleation. In addition, the curves of three different tests are plotted for each diameter showing high repeatability.

Figure 5.7(b) shows the force-displacement curves of the normal tests considering three tests for each weld diameter. The typical curves related with not brittle fracture already seen in Figure 4.12 for the 22MnB5 joints normal tests can be seen here. An influence of the spot weld dimension on the onset of crack forces is observed. Another important point is that the maximum forces are higher than those of $22 \mathrm{MnB} 5$ joints of the same thickness combinations presented in Figure 4.12. The force-displacement curves of the mix-mode tests are presented as well in Figure 5.7(b). This graph only shows one tested thickness combination which was welded with an unique set of welding parameters. Within all tests there was a good level of repeatability, as well as a clear lineal behavior of the joint up to maximum force, when brittle fracture occurs.

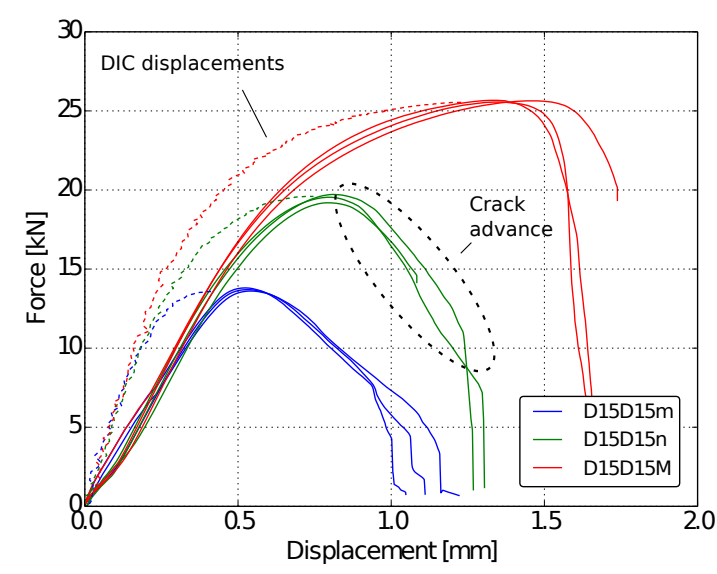

(a) Shear tests.

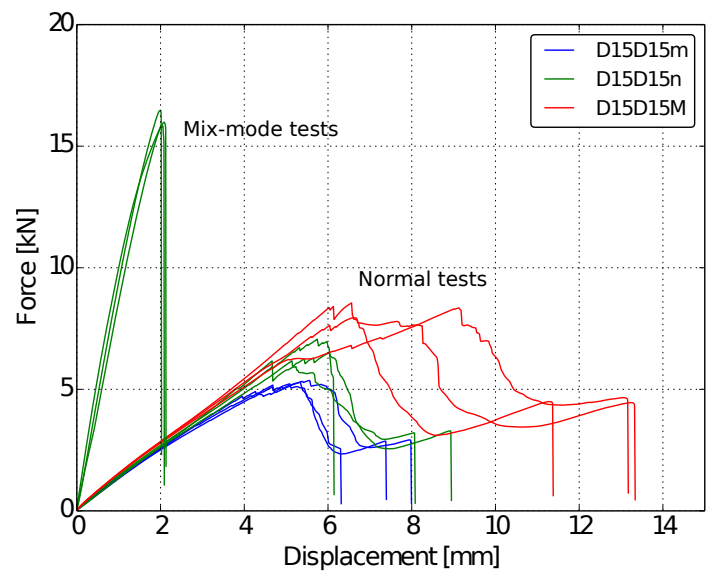

(b) Mix-mode and normal tests.

Figure 5.7: Force-displacement curves of DP980 joints. Dot lines represent one test for each combination in shear tests, where displacements where measure with DIC device. 
5.4. Experimental loading tests of the joining specimens

\subsubsection{Fracture analysis}

The final appearance of the failed spot welds are analyzed to determine the onset of crack. The same fracture modes detected in 22MnB5 joints are observed in the DP980 joints: All shear and mix-mode tests present a FIF mode with clear shear mode II fracture surface as presented in Figure 5.8. This surface indicates that fractures starts at the weld notch and cross the nugget splitting it into two parts.

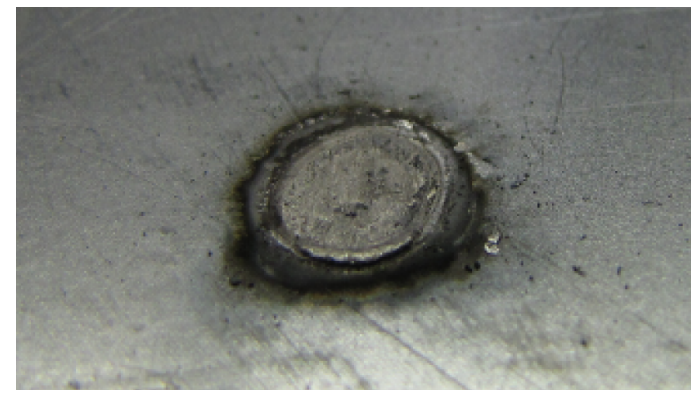

(a) Shear test.

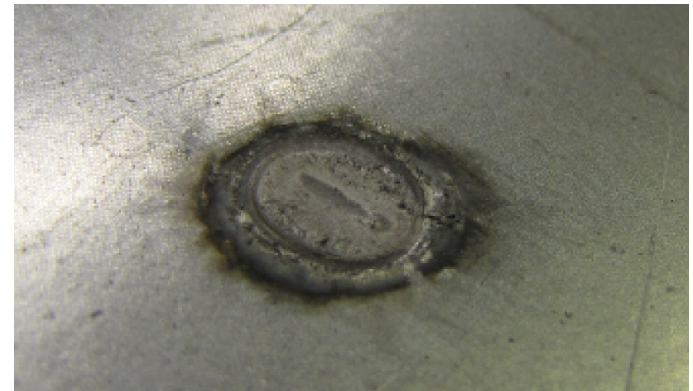

(b) Mix-mode tests.

Figure 5.8: Top view of failed spot welds at Full Interface Failure mode of DP 980 similar joints.

However, normal tests present extremely irregular failed spot welds. A three dimensional crack propagation path has been seen in these joints beginning always from the weld notch and causing the appearance of failed welds to present a singular geometries. Some failed joints show the common PIF fracture (see Figure 5.9(a) and Figure 5.10(a) , where the spot weld material stays on one of the sides. Other joints present a double button pull out failure (DBPF) appearance (see Figure 5.9(b) and Figure 5.10(b)]. In these joints crack front has advanced first from the notch in the faying surface direction from both sides of the weld towards the nugget center, however, at some point this direction changed and the crack continued outwards in opposite directions for each side of both sheets. The direction that is taken by the crack front depends on small inhomogeneities of the nugget material and is therefore not repeatable. For example, for the same thickness combinations and same set of welding parameters different failed spot weld appearance have been noticed at different test repetitions. These different crack front evolutions lead to a low repeatability of the force-displacement curves after the first onset of crack as observed in Figure $5.7(\mathrm{~b})$. 


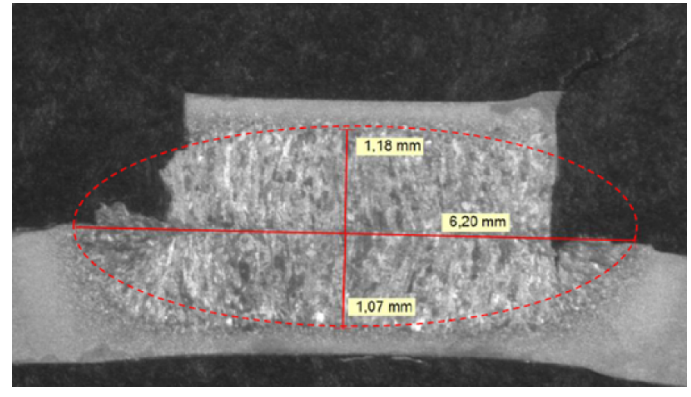

(a) PIF.

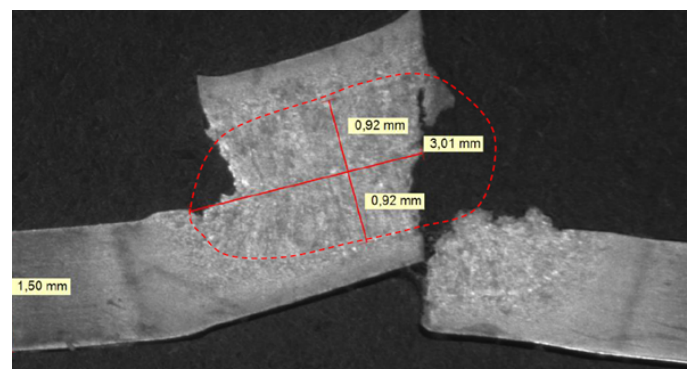

(b) DBPF.

Figure 5.9: Cross sectioned failed spot welds of normal DP 980 similar joints.

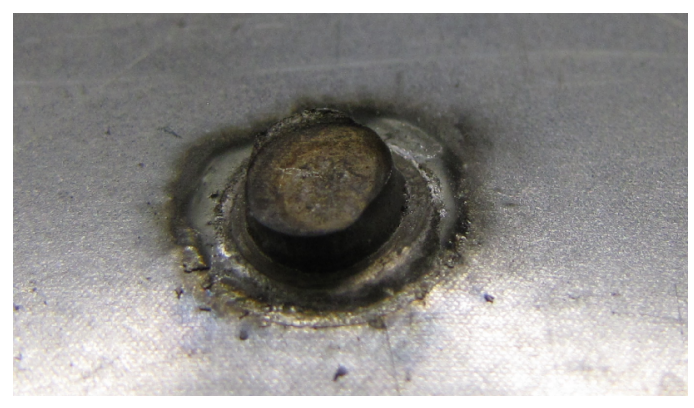

(a) PIF.

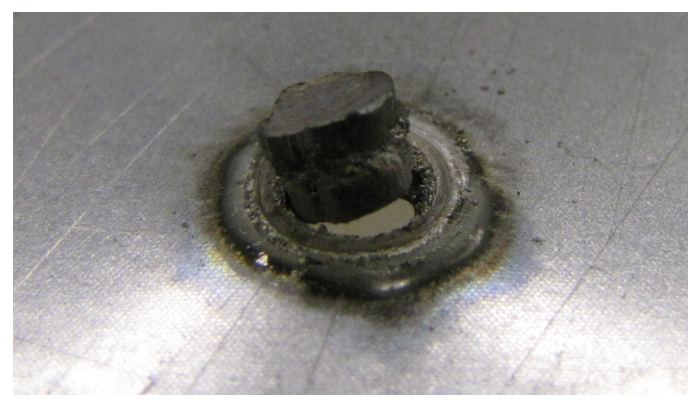

(b) DBPF.

Figure 5.10: Top view of failed spot welds of normal DP 980 similar joints.

The failure modes for all loading cases are presented in Table 5.3. As general conclusion, for all combinations the joint failure starts at the weld notch, even considering the multiple failed weld appearance of the normal tests.

\begin{tabular}{lccc}
\hline Joint code & Shear & Mix-mode & Normal \\
\hline D15D15m & FIF & $* * *$ & PIF-DBPF \\
D15D15n & FIF & FIF & PIF-DBPF \\
D15D15M & FIF & $* * *$ & PIF-DBPF \\
\hline
\end{tabular}

Table 5.3: Failure modes for each thickness combination, weld diameter and loading mode of similar DP 980 joints. 


\subsection{Model validation}

The validation of the extended spot weld model is presented in this section. The particular features of the DP 980 joints that were characterized in the previous sections are applied to the model in Section 5.5.1. The simulated results are compared with the experimental ones in Section 5.5.2.

\subsubsection{Detailed model features}

The similar DP 980 joints are modeled using the same FEM model regarding the constitutive model and the mesh description presented for similar 22MnB5 joints in Sections 3.1 and 4.5.1. The only difference when comparing with the 22MnB5 joints is the applied strain hardening curves for the multiple welding zones. Figure 5.11 shows a scheme of the material properties distribution applied for this type of joint based on the hardness profiles depicted in Figure 5.2 . For the BM with a dual phase microstructure the properties of the DP 980 base material are considered. For the FZ, the hardening curves obtained with the FEM optimization procedure in Section 4.5.1 are applied. The $\mathrm{HAZ}$ is divided in two parts: the hardness drop observed in SHAZ is neglected and the strain hardening curve of the DP 980 BM is applied in this zone. For the HHAZ with a martensitic hard microstucture, the a plasticity model of the FZ is applied.

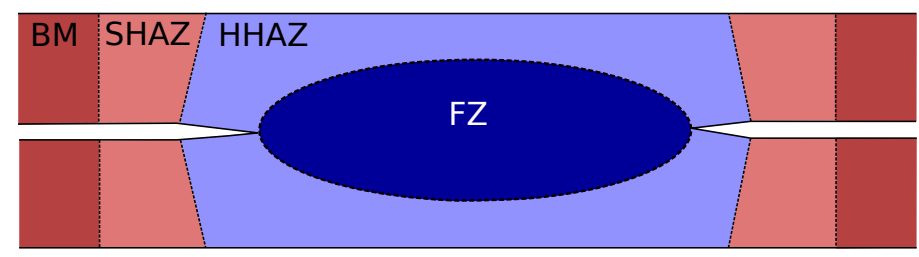

Figure 5.11: Schematic representation of the welding zones under plasticity modeling point of view. The weld is modeled using two different hardening curves, one for BM and SHAZ and a different one for HHAZ and FZ.

The $J$-integral is used as fracture criterion, based on the fracture analysis given in Section 5.4.2. J-integral is evaluated in the critical section of the weld notch, and in the faying direction, i.e. parallel to the sheet plane. 


\subsubsection{Force-displacement curves and maximum forces validation}

Figure 5.12(a) shows the simulated and experimental results of the shear tests considering multiple weld sizes. Both experimental and simulated curves present the same shape and reach similar maximum forces, only some slight differences are observed in the displacements due to measurement errors. Same critical $J$-integral for mode II failure as in the similar $22 \mathrm{MnB} 5$ joints (i.e. $J_{I I C}=56 \mathrm{~kJ} \mathrm{~m}^{-2}$ ) is used here to calculate the maximum forces.

The same difficulties with reproducing the boundary conditions are observed in Figure 5.12(b) for mix-mode tests. The maximum forces are well estimated, however the force-displacement curves are not fitted exactly due to the inexact reproduction of the lateral displacements observed in the real experiments. In the same way as in $22 \mathrm{MnB} 5$ mix-mode test, mode II critical value of $J$-integral is used, i.e. $J_{I I C}=56 \mathrm{~kJ} \mathrm{~m}^{-2}$.

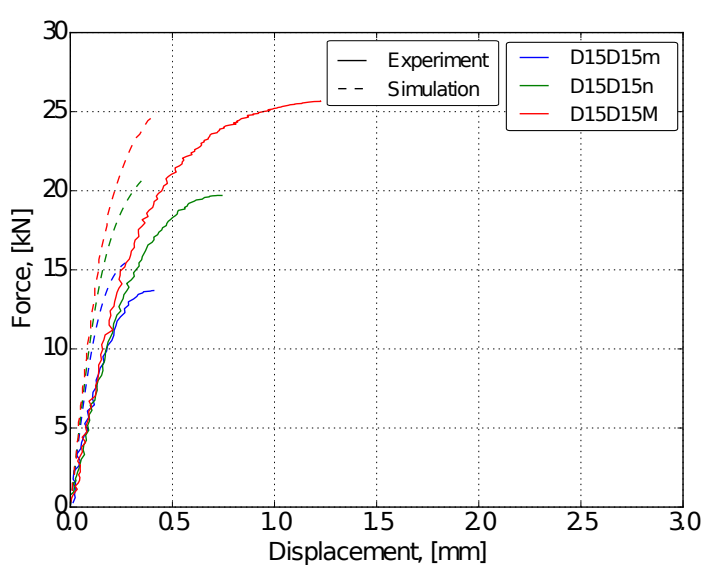

(a) Shear tests.

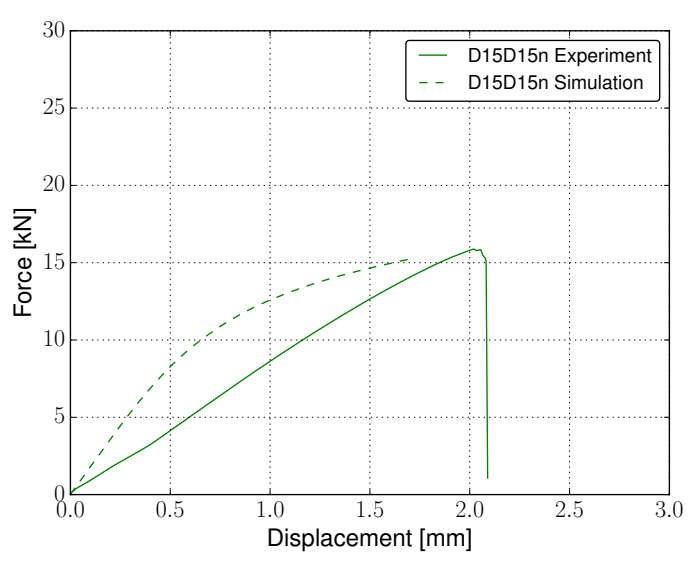

(b) Mix-mode tests.

Figure 5.12: Comparative between calculated and experimental force-displacement curves of similar DP 980 joints. Notice that the discrepancies in the displacements of the shear tests are caused by sliding of the specimen from the Zwick machine clamping device that were corrected in the rest of tests presented in this work.

In the Figure 5.13(a) the force-displacement curves of the normal tests are plotted. Some discrepancies can be seen in the displacements as in the case of 22MnB5 tests caused by the unreliable boundary conditions. However, if the same critical $J$-integral is used as the $22 \mathrm{MnB} 5$ joints normal tests (i.e., $J_{I C}=22.5 \mathrm{~kJ} \mathrm{~m}^{-2}$ ), then 
the maximum force is clearly underestimated. A possible explanation is the different fracture toughness between DP 980 and 22MnB5 nugget material due to the differences in the carbon contain. Previous works have reported a strong discontinuity in fracture toughness of martensitic steels in the Vickers range of 400 to 500 [84], this range includes, respectively the DP 980 and the 22MnB5 nuggets as observed in Figure 4.5 and 5.2 , and consequently their fracture toughness in mode I is different. This martensitic nugget material fracture toughness variation leads to different higher bearing forces.

In order to get an approximation of mode I fracture toughness, critical $J$-integral is estimated by using an inverse engineering approach. This approach considers the maximum forces obtained in the experimental normal tests, and intersecting this value with the $J$-integral evolution curve in order to obtain the desired value. As a first approximation, $J_{I C}=53 \mathrm{~kJ} \mathrm{~m}^{-2}$ is considered the most adequate value, but it must be pointed out that the maximum experimental forces presents high scatter, leading to low accuracy in the obtained critical $J$-integral value.

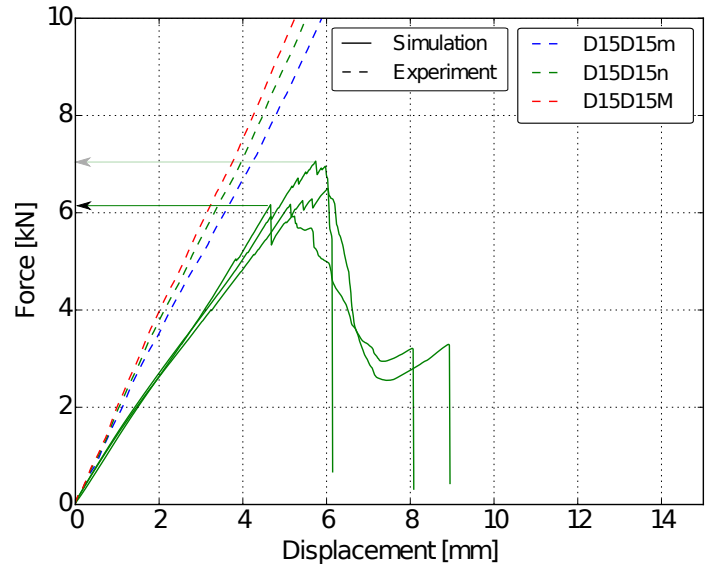

(a) Force-displacement curves.

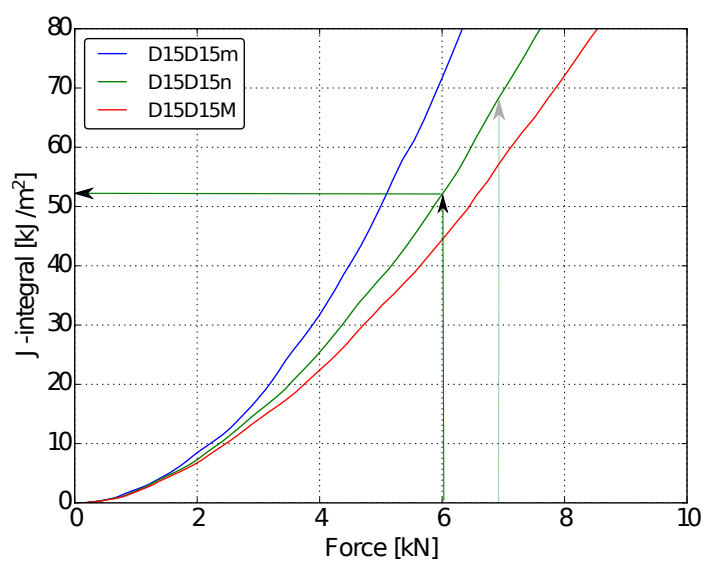

(b) J-integral evolution.

Figure 5.13: Experimental and simulated results of normal tests for DP980 joints. Critical $J$-integral is obtained using inverse engineering from the experimental maximum forces. The scattering of the experimental force-displacement curves introduce variation in the obtained critical value.

To extensively analyze the critical values of $J$-integral for mode I and mode II, the experimental and the simulation maximum forces are plotted versus the weld diameters of the joints. In the case of shear tests (see Figure 5.14(a)), all experimental forces are predicted accurately using the same critical $J$-integral used for 22MnB5 
joints. Strong sensitiveness to spot weld diameter can be observed and the slight differences between simulation and experiments come consequently from the weld diameter measurement inaccuracy.

For the normal tests, the Figure 5.14(b) shows lower weld diameter sensitivity than the shear tests case both in the experimental and in the simulation results. As presented in Figure 5.13 , the critical $J$-integral mode I value presented for 22MnB5 joitns, i.e. $J_{I C}=22.5 \mathrm{~kJ} \mathrm{~m}^{-2}$, and a value of $J_{I C}=53 \mathrm{~kJ} \mathrm{~m}^{-2}$ fits the multiple experimental values better. However, it underestimates the critical forces of larger welds.

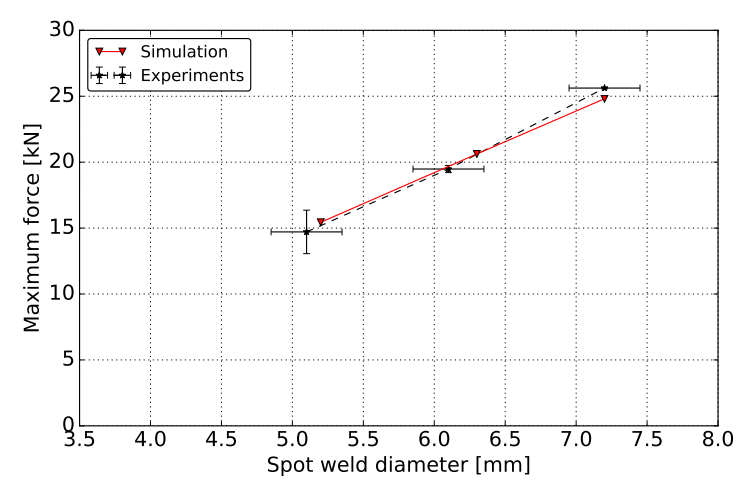

(a) Shear tests.

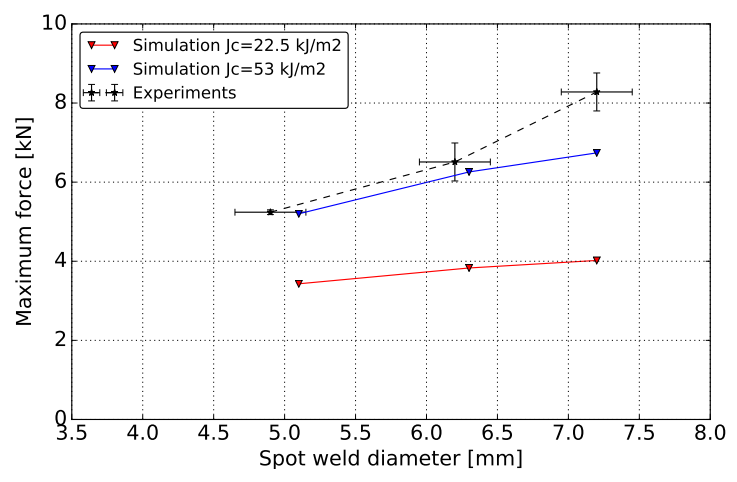

(b) Normal tests.

Figure 5.14: Maximum forces represented as a function of the weld diameter in case of DP980 shear tests. The experimental results are plotted with error bars for the forces representing the standard deviation and error bars of diameter considering the measurement errors of $\pm 0.2 \mathrm{~mm}$. 


\section{Chapter 6}

\section{Application in resistance spot welds of two different steel grades}

In the previous chapters of this thesis a spot weld FE model has been developed for joints of two sheets of the same steel. However, resistance spot welds joining two different steel grades can be usually found in a car structure. These types of joints are analyzed in this chapter, considering the two steel grades presented in the the previous chapters, the hot formed martensitic boron steel (22MnB5) and the cold formed dual phase steel (DP 980).

A cognate approach to the one followed for the similar joints is presented in this chapter. An experimental campaign considering multiple thickness combinations, weld diameters and loading angles is designed in Section 6.1. The joints are analyzed in detail using cross sections in Section 6.2 in order to identify the geometric features, the welding zones dimensions and the material distribution properties of these types of joints. Following the same procedure presented for DP 980 similar joints the calibration of the plasticity model parameters of the main welding zones is done in Section 6.3. The experimental loading test results are presented in Section 6.4. Finally, a comparison between experimental and simulated results is shown in Section 6.5. 
6. Application in RESistance spot Welds of TWO DifFERENT STEEL GRADES

\subsection{Experimental validation campaign}

In order to analyze the influence of the sheet thickness ratio, three thickness combinations are used (see Table 6.1). In all cases the DP 980 sheet has a constant thickness of $1.5 \mathrm{~mm}$. There are three different thickness dimensions for the 22MnB5 sheets, a low value of $0.8 \mathrm{~mm}$, a medium value equivalent of $1.5 \mathrm{~mm}$ and a maximum value of $2.0 \mathrm{~mm}$. Notice that the same thickness combinations have been analyzed for joints of 22MnB5 sheets (see Table 4.2). The sets of welding parameters (depicted in Table 6.2 are chosen to obtain the desired nominal weld diameters, $d_{n}$. In specimens with both sheets of the same thickness combination of $1.5 \mathrm{~mm}$, three sets of welding parameters have been applied to obtain multiple weld diameters.

\begin{tabular}{l|cc|cc|cc}
\hline \multicolumn{2}{c}{ Joint } & \multicolumn{2}{c}{ Sheet 1 } & \multicolumn{2}{c}{ Sheet 2 } & \multicolumn{2}{c}{ Diameter $(\mathrm{mm})$} \\
Code & Mat & $t_{1}(\mathrm{~mm})$ & Mat & $t_{2}(\mathrm{~mm})$ & Target & Obtained \\
\hline U08D15n & 22MnB5 & 0.8 & DP 980 & 1.5 & $d_{n}=4.4$ & $4.80 \pm 0.2$ \\
U15D15m & 22MnB5 & 1.5 & DP 980 & 1.5 & $d_{m}<5.5$ & $5.00 \pm 0.2$ \\
U15D15n & 22MnB5 & 1.5 & DP 980 & 1.5 & $d_{n}=5.5$ & $6.50 \pm 0.2$ \\
U15D15M & 22MnB5 & 1.5 & DP 980 & 1.5 & $d_{M}>5.5$ & $7.00 \pm 0.2$ \\
U20D15n & 22MnB5 & 2.0 & DP 980 & 1.5 & $d_{n}=5.7$ & $6.20 \pm 0.2$ \\
\hline
\end{tabular}

Table 6.1: Sheet and material thickness combinations and corresponding target and obtained nugget diameter in 22MnB5 and DP 980 dissimilar joints.

\begin{tabular}{l|c|cc|ccccc}
\hline \multicolumn{1}{c}{ Joint } & Force $(\mathrm{kN})$ & \multicolumn{2}{c}{ Intensities $(\mathrm{kA})$} & \multicolumn{5}{c}{ Time $(\mathrm{ms})$} \\
Code & $\mathrm{F}$ & $I_{1}$ & $I_{2}$ & $t_{s q}$ & $t_{s 1}$ & $t_{f 1}$ & $t_{s 2}$ & $t_{f 2}$ \\
\hline U08D15n & 3.00 & 3.00 & 5.10 & 1000 & 200 & 20 & 400 & 200 \\
U15D15m & 3.00 & 3.00 & 4.70 & 500 & 200 & 20 & 400 & 200 \\
U15D15n & 3.00 & 3.00 & 6.60 & 500 & 200 & 20 & 400 & 200 \\
U15D15M & 3.00 & 3.00 & 7.20 & 500 & 200 & 20 & 400 & 200 \\
U20D15n & 4.00 & 3.00 & 6.20 & 1000 & 200 & 20 & 400 & 200 \\
\hline
\end{tabular}

Table 6.2: Welding parameters for all material, thickness and target diameter combinations in 22MnB5 and DP 980 joints. 


\subsection{Geometry and local material properties distribution of the spot welds}

Welded joints are cross sectioned in order to analyze the geometrical features and the welding zones. Two of these cross sections are presented in Figures 6.2 following the same procedure presented in Section 4.2.1. The observed spot weld geometry is cognate to the one of similar joints showed in Section 4.2.2 . The center of the fusion zone is situated in the middle point of both sheets and the lighter stripes demarcate the HAZ,

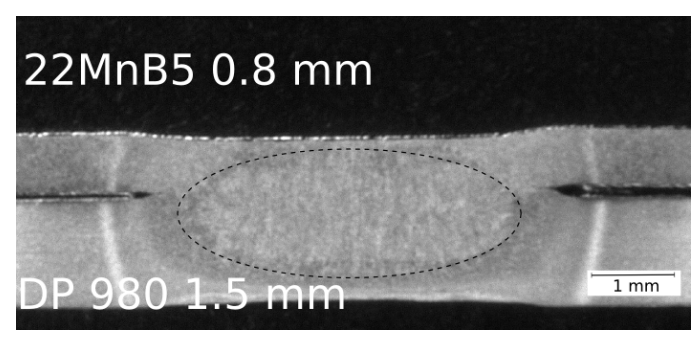

(a) U08D15n.

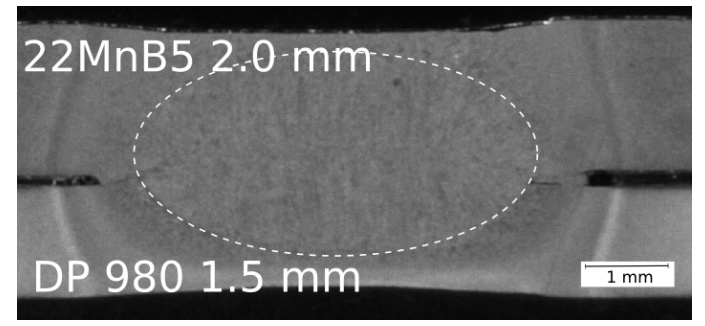

(b) U20D15n.

Figure 6.1: Cross sectioned spot weld general view of not failed dissimilar joints.

The same hardness line measurements are carried for all the thickness combinations depicted in Table 6.1, which use the same procedure presented in Section 4.2.1. The hardness line starts from the DP 980 material, cross the nugget and finishes in the 22MnB5 material. Figure 6.2(a) shows the hardness profiles of the three combinations. The shape of the profile is similar for all combinations. However, the scattering in the values does not allow for a clear analysis of the values. An idealized scheme of the hardness line is presented in Figure 6.2(b) in order to clarify the hardness zones. In between the hardness measurement points that are done at both materials, the fusion zone values can be seen. These points have a constant hardness value for each combination, that is in between the values of FZ hardness of similar joints of both joined materials (see Figure 4.5 and Figure 5.2. The fact that the FZ hardness is constant for all measurement points indicates that both material are completely melted during the welding process. Although it is slightly appreciated due to measurement scattering, it can be seen that in combinations with a higher proportion 22MnB5 material, i.e., in which 22MnB5 sheet is thicker (U20D15), the FZ hardness is slightly higher. This can be explained by taking into account that 


\section{APPLICATION IN RESISTANCE SPOT WELDS OF TWO DIFFERENT STEEL}

GRADES

the hardness value of martensite is strongly dependent on the carbon contain of the steel. Consequently, the higher the 22MnB5 content in the mixed material of the nugget is, the higher the amount of carbon is and consequently the hardness value also increases.

It must be noted that a clear hardness drop can be seen in the nugget boundary for some of the tested combinations. This hardness drop has been already reported in the similar 22MnB5 combinations (see Section 4.2.3) and may be an indicator of lower mechanical properties in a highly confined region surrounding the nugget.

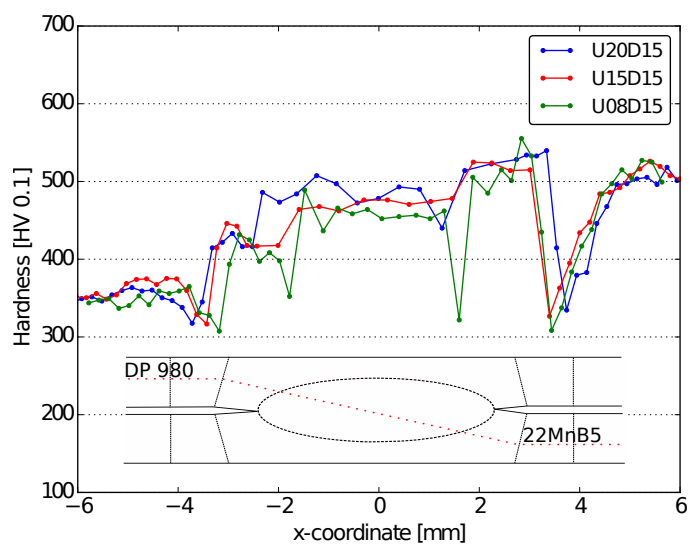

(a) Measurements.

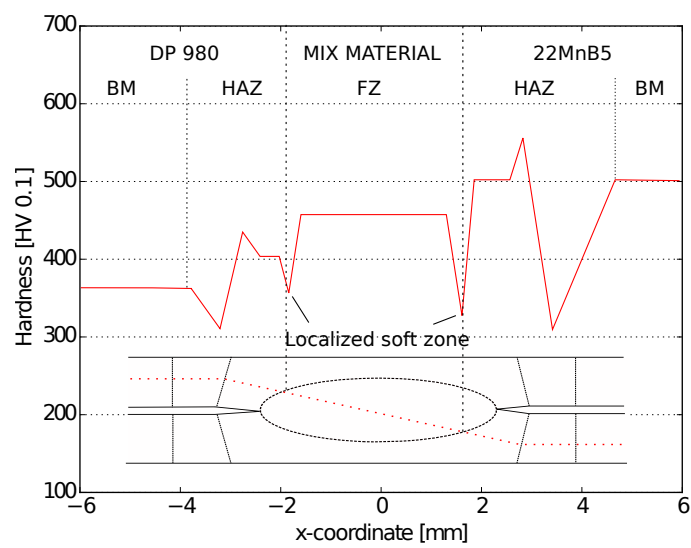

(b) Scheme.

Figure 6.2: Hardness measurements of DP 980 and 22MnB5 joints with different thickness combinations. The left side corresponds to DP 980 related material, including BM and HAZ. The right side shows the hardness profile of fully hardened 22MnB5 HAZ and BM. Note that spot weld diameters are not the same for all combinations.

In conclusion, the material mechanical properties of the weld zones of dissimilar joints can be extracted from the properties of the similar joints, which have been already characterized in the previous chapters of this thesis. Only the mixed fusion zone strain hardening behavior must be calibrated in order to define the detailed $\mathrm{FE}$ model of the dissimilar joints. This procedure is presented in the next section. 


\subsection{Characterization of nugget material properties}

In order to calibrate the hardening curves of dissimilar joints nuggets, it is necessary to test of micro tensile welded specimens. The manufacturing and the results of these mechanical micro tensile tests are presented in Section 6.3.1. A method to obtain the hardening curves of the nuggets in dissimilar joints is presented in Section 6.3.2.

\subsubsection{Mechanical tests}

Micro specimens are manufactured with mixed weld material in the gauge zone in order to evaluate the plasticity of nugget material. For this purpose, a similar procedure to that used in Section 4.3 is used in this chapter to obtain these specimens. In this case, the sacrificial coupon is DP 980 material with $1.5 \mathrm{~mm}$ thickness. It is welded onto a $130 \times 60 \times 1.5 \mathrm{~mm}$ coupons of 22MnB5 material using the welding parameters of combination U15D15M on Table 6.2. The coupon specimens are removed afterwards using EDM and the PST and UT geometries are cut in the 22MnB5 sheet. Therefore, the resulting specimens are constituted of 22MnB5 material and have a mix nugget material in the gauge zone (see Figure 6.3). Five specimens of both geometries are loaded following the process introduced in Section 4.3.2.

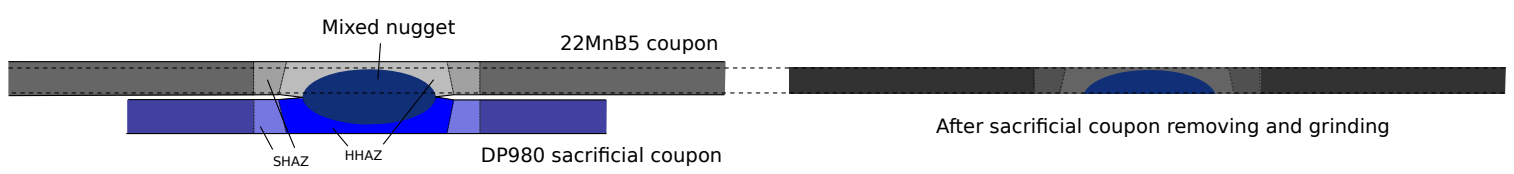

Figure 6.3: Scheme of cross sectioned spot weld used for the nugget material characterization of the dissimilar joints. A sacrificial DP 980 coupon is welded onto the $22 \mathrm{MnB} 5$ coupon, after this sacrificial coupon is removed and ground, the mixed nugget material (dark blue) remains in the center of the specimen.

Consistent repeatability can be observed on the force-displacement curves presented in Figures 6.4, in which the present section's specimens are labeled as mixed weld. The previously discussed micro specimen curves with welded material in the gauge zone from similar joints are plotted as well in these figures. The same curve shapes can be observed for all cases due to the martensitic microsture of the fusion zones of all tested combinations. The increase of maximum force is related with 


\section{APPliCATiOn in RESistance SPOT WELDS OF TWO DifFERENT STEEL}

\section{GRADES}

the carbon contain of the different nugget materials, which was analyzed in the Section 6.2. The lower is the carbon contain of the martensitic nugget material, the lower is the ultimate strength of this material leading to a decrease of the reached maximum forces.

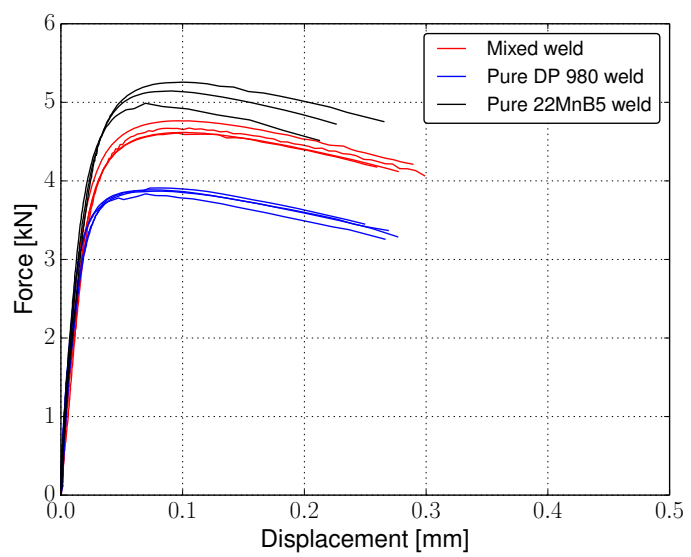

(a) PST

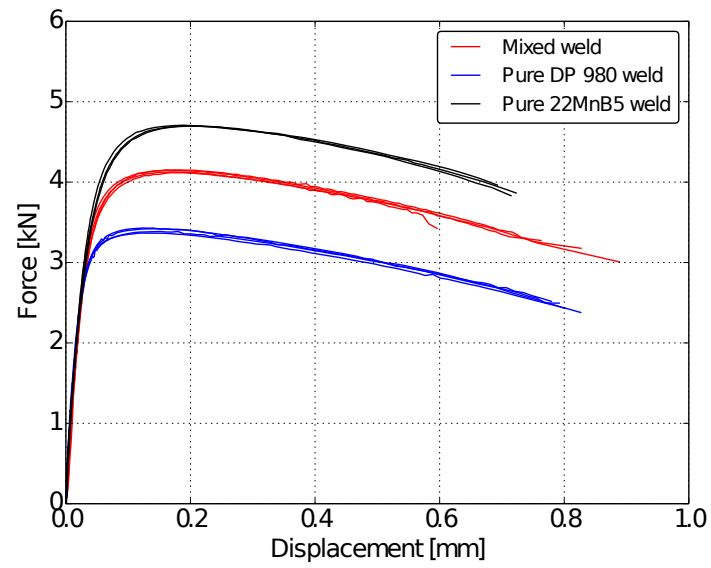

(b) UT

Figure 6.4: Reduced tensile tests with spot weld microstucture in the gauge zone extracted from similar and dissimilar joints. Mixed weld indicates the results of specimens welded with a DP980 sacrificial coupon as presented in Figure 6.3.

\subsubsection{Plasticity model calibration}

In order to obtain the plasticity model of dissimilar joints nugget material two approaches are proposed in this chapter: Either extracting it from the experimental tests showed in the previous section using an inverse FEM optimization scheme already presented in Section 5.3.2, or using a interpolation mixed rule considering the hardening curves in the fusion zones of welded joined materials.

First, the inverse FEM optimization scheme is used to obtain the strain hardening curves of the nugget material from the force-displacement curves. FEM simulated force-displacement curves of the PST and UT tests are compared with the experimental results presented in the previous section. The FEM model is divided in two zones. One of the zones has the properties of $\mathrm{HAZ}$ and $\mathrm{BM}$ of the similar 22MnB5 joints and the other zone has the properties of the mixed FZ that must be fitted. The plastic model, i.e. the parameters of Swift-Voce law (see Eq. 5.1), of the second zone are changed iteratively up to the point of fitting the experimental results. The 
obtained optimized hardening curves are presented in Figure 6.5. These curves are compared with the hardening curves obtained for 22MnB5 and DP 980 nugget material in the previous chapters of this thesis and previous works [18]. As expected, the curves of the mix material nugget are approximately in the middle point between both pure nugget materials. This phenomenon can be clearly observed for the small strains in Figure 6.5(a), but a clear divergence can be found in the large strains in Figure 6.5(b).

The second approach consists of calculating the hardening curve of the nugget zone of dissimilar joints using the rule of mixtures, i.e. wighted mean of the nugget hardening curves of the joined materials using the thickness ratio as a weighting parameter:

$$
\sigma_{k}^{M I X}\left(\bar{\varepsilon}_{p}, x_{D}\right)=x_{D} \cdot \sigma_{k}^{D}\left(\bar{\varepsilon}_{p}\right)+\left(1-x_{D}\right) \cdot \sigma_{k}^{U}\left(\bar{\varepsilon}_{p}\right)
$$

where $\sigma_{k}$ indicates the strain hardening curve and the subindex ${ }_{D}$ and ${ }_{U}$ refer to DP 980 and 22MnB5 material respectively. $x_{D}$ is the weighting parameter and is calculated using $x_{D}=\frac{t_{D}}{t_{D}+t_{U}}$, where $t$ indicates the sheet's thickness. In the case of joints with same thickness combination, the mixed curve is the mean of both pure joint curves. The results obtained differ only slightly for small strains to those obtained using the FEM inverse optimization as observed in Figure 6.5(a).

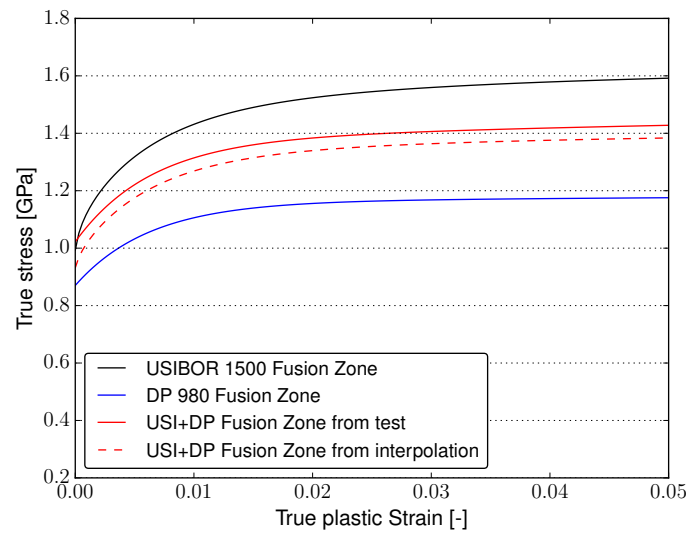

(a) Small strains.

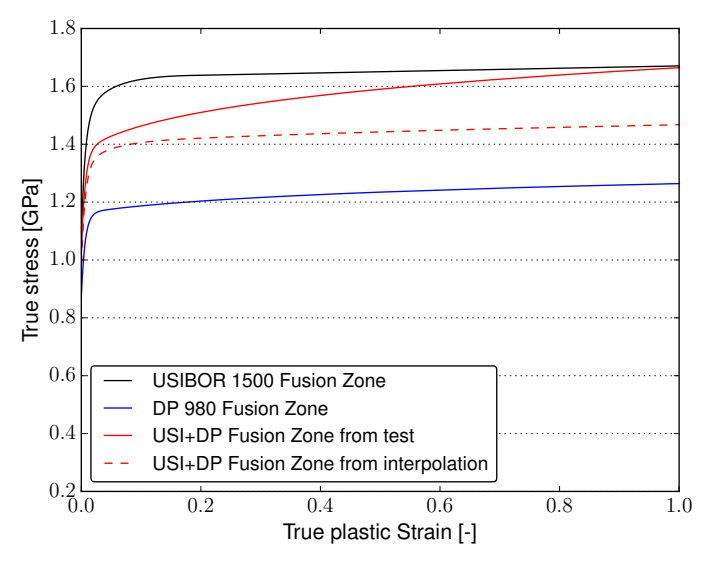

(b) Large strains.

Figure 6.5: Strain hardening curves of the weld nugget material for different steel grades combinations. 


\subsection{Experimental loading tests of the joining specimens}

The joints presented in Table 6.1 are loaded following the same procedure followed in Section 4.4.1. Five repetitions are done for each combination.

\subsubsection{Force-displacement curves}

Figure 6.6(a) shows the force-displacement curves of the shear tests. The most representative curve is plotted for each one of the multiple thickness combinations and the weld sizes. Brittle fracture curves appear in almost all combinations (except for U08D15n) as seen before in the previous chapters for all shear tests curves. U08D15n presents a slightly different fracture mode that is detailed in Section 6.4.2. Similar loading slopes are observed in all cases, the slight differences of tests U20D15n derives from a measurement error. Analyzing the maximum force for each test reveals two groups of curves, those of U08D15n and U15D15m, and those of U15D15n, U15D15M and U20D15n. As with the material combinations presented in the previous chapters the explanation for the appearance of these two groups is evident from the obtained weld diameters of each combination (see Table 6.1). The first curves are related to welds of less than $5 \mathrm{~mm}$ and the latter curves of those of more than $6.5 \mathrm{~mm}$.

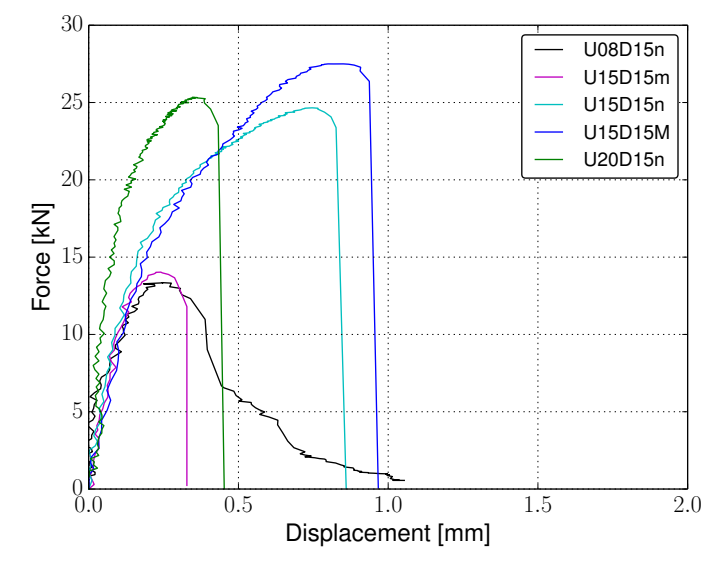

(a) Shear tests.

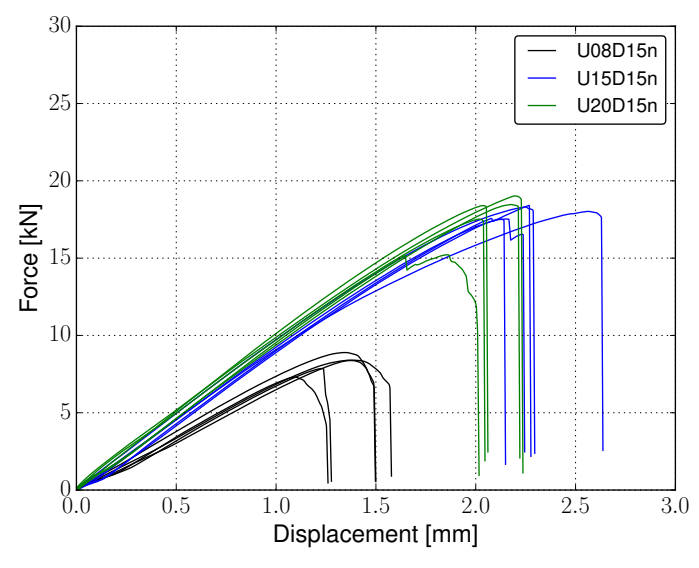

(b) Mix-mode tests.

Figure 6.6: Force-displacement curves of shear and mix-mode tests for dissimilar DP980 and 22MnB5 joints. DIC displacement measurments are considered in all curves. 
In the same way that Figure 6.6(a) explains shear tests, Figure 6.6(b) shows the force-displacement curves of the mix-mode tests. Five curves are plotted for each combination presenting high repeatability. Linear loading curves up to brittle fracture are observed in all curves. Again, the joints with larger diameters show a higher maximum force (U15D15n and U20D15n).

The results or normal tests are plotted in Figure 6.7(a). Three curves are plotted for each thickness combination. The curves have different slopes due to the bending displacement of the sheet around the weld as observed in the similar 22MnB5 joints. A significant lower maximum force is observed in the combination with a thinner sheet, i.e. U08D15n. The force-displacement curves of normal tests with multiple weld diameters are presented in Figure 6.7(b). While the sensitivity of the maximum force to the weld size is not that high as in the shear tests case, slight differences are observed. Notice that because the curves scatter these differences can be seen only in a qualitative way. In summary, the obtained results for shear, normal and mix-mode tests are comparable to those obtained for similar joints in terms of loading and fracture response.

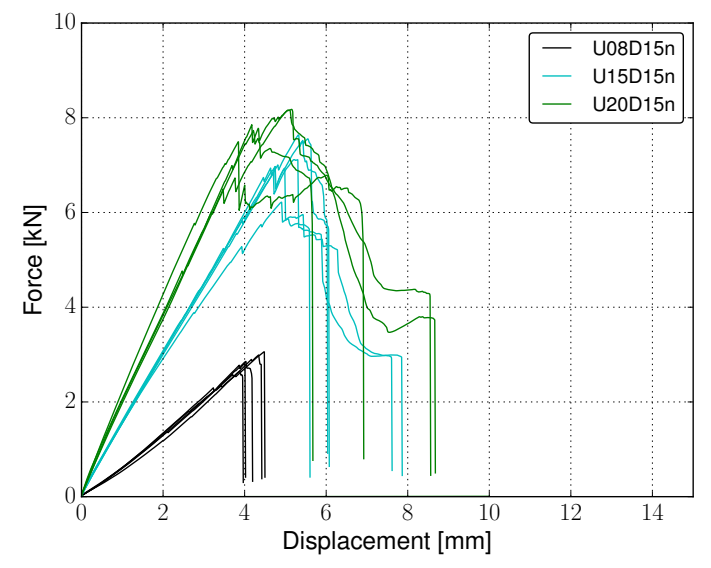

(a) Different thickness combinations.

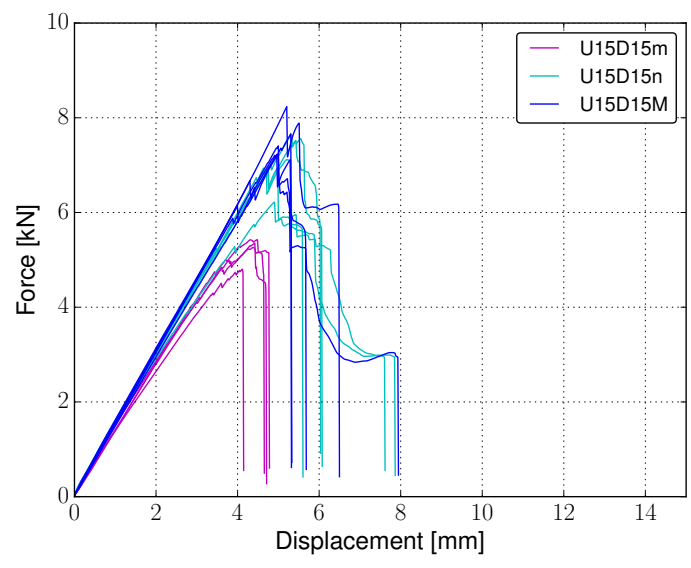

(b) Different spot weld diameters.

Figure 6.7: Force-displacement curves of normal tests of dissimilar joints.

Finally, Figure 6.4.1 shows a comparison of the same loading tests applied in joints of the material combinations tested in this thesis, with the same thickness combination (two sheets of $1.5 \mathrm{~mm}$ ) and a similar weld size, i.e. U15U15n with a weld diameter of $5.00 \pm 0.2 \mathrm{~mm}$, D15D15m of $5.10 \pm 0.2 \mathrm{~mm}$, and U15D15m of $5.00 \pm 0.2 \mathrm{~mm}$. Similar maximum forces are observed for the three combinations in 


\section{APPliCATiOn in RESistance SPOT WELDS OF TWO DifFERENT STEEL}

\section{GRADES}

the case of shear tests in Figure 6.8(a). One of the differences is the curves of similar DP 980 joints show no brittle fracture, due to the stable crack advance. Furthermore, the maximum force of similar 22MnB5 joint are roughly higher, however, it should be noticed that the weld size and its inaccurate value may have an influence on this different bearing capacity. Figure 6.8(b) shows the normal tests results. Lower onset of crack forces are observed for the similar 22MnB5 joints. This is in accordance with the fact that those nuggets with higher hardness, i.e. those where DP 980 material is involved, have higher fracture toughness in mode I fracture mode.

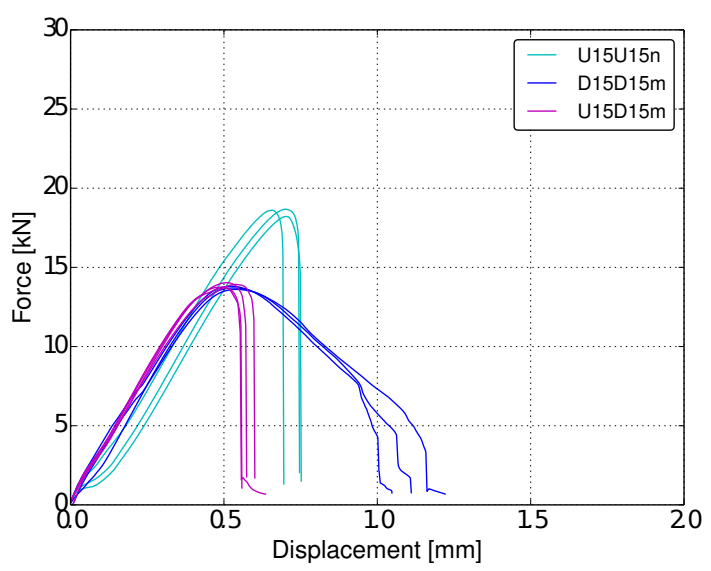

(a) Shear tests.

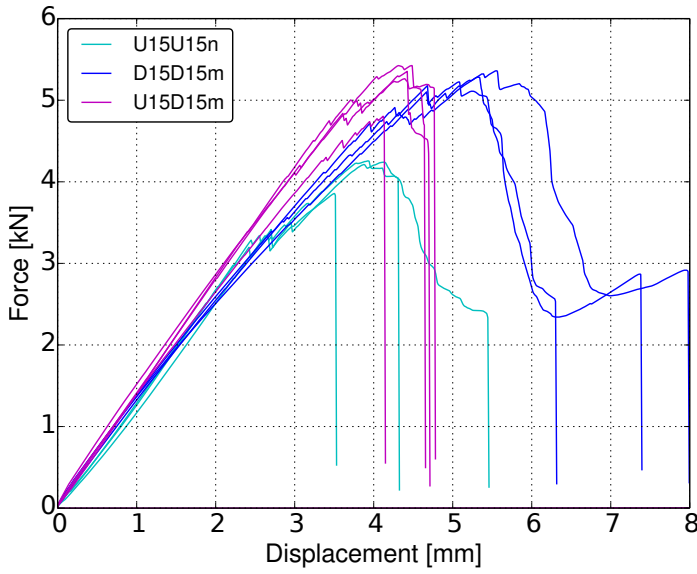

(b) Normal tests.

Figure 6.8: Force-displacement curves of joints with same thickness combination, similar weld sizes and different material combinations.

\subsubsection{Fracture analysis}

The failure modes of all tested joints following the clasification of Figure 2.9 are presented in Table 6.3. For shear and mix-mode tests FIF is observed in almost all combinations (see example in Figure6.9(a)p. It leads to brittle failure curves and spot weld brittle fracture surfaces. However, in the U08D15n joint, the crack front propagation has surrounded the nugget leading to PDF mode as shown in 6.9(b), The nugget has stayed attached to the thicker sheet, i.e. the DP 980 sheet. This attachment is due to the proximity of crack tip to nugget top surface (see Figure 6.1(a)), where lower extremely localized hardness values are measured as observed in Figure 6.2 , 
6.4. Experimental loading tests of the joining specimens

\begin{tabular}{lccc}
\hline Joint code & 00 deg & 45 deg & 90 deg \\
\hline U08D15n & PDF & FIF & PDF \\
U15D15m & FIF & $* * *$ & FIF-PIF \\
U15D15n & FIF & FIF-PDF & PIF \\
U15D15M & FIF & $* * *$ & PIF \\
U20D15n & FIF & FIF & PIF \\
\hline
\end{tabular}

Table 6.3: Failure modes for each thickness combination, weld diameter and loading mode of dissimilar DP980 and 22MnB5 joints.

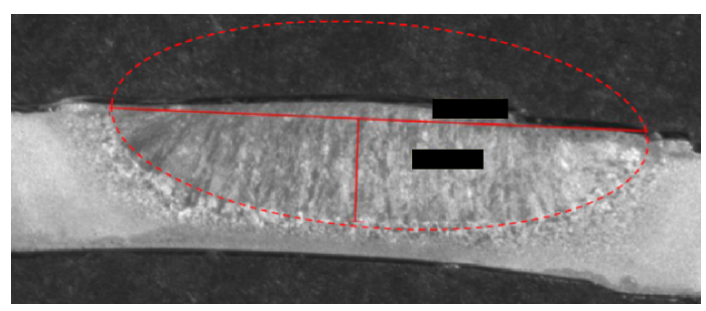

(a) FIF in U20D15n shear test.

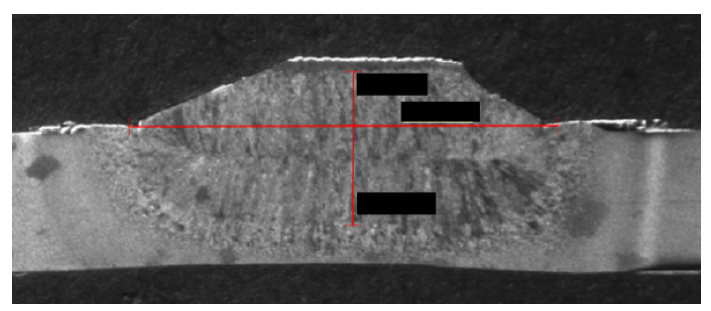

(b) PDF in U08D15n shear test.

Figure 6.9: Cross-sectioned failure spot welds of dissimilar joints shear tests.

In normal tests, the two failure modes are observed, TDF/PDF and PIF/FIF, both presenting ductile fracture surfaces. For the combination U08D15n, clear PDF is observed, the crack front propagates along the nugget boundary until some point when it kinks and advances towards the outward surface of the thinner sheet (see Figure 6.10(a)d. Consequently the nugget stays attached to the DP 980 sheet. The explanation for this fracture path is the nugget boundary confined soft zone in the same way that for the shear tests case. In the case of similar thickness combinations and small diameters, such as U15D15m, the crack propagates in the faying surface direction up to the nugget center proximities where it kinks in some tests leading to PIF. Either PIF or FIF appear in the failed joints depending on the crack kinking. A clear ductile surface is formed in the faying plane as shown in Figure 6.11(a). The remaining weld material stays in side of the dual phase material. For a larger diameter, the fracture begins in the notch as well, and propagates in the faying plane direction, however it kinks earlier and a greater portion of the welds stays on side of the joints (see Figure 6.11(b)). This crack path gives a different final appearance of the weld, similar to the BPO failure typical of the ductile steels, however they are technically PIF where the rupture starts from the notch. Finally, in the U20D15n 


\section{APPliCATiOn in RESistance SPOT WELDS OF TWO DifFERENT STEEL}

\section{GRADES}

combination, the crack advances through the nugget in the faying direction and kinks to the thinner sheet, i.e. DP 980 sheet. The remaining weld material stays on the 22MnB5 side (see Fig 6.10(b)).

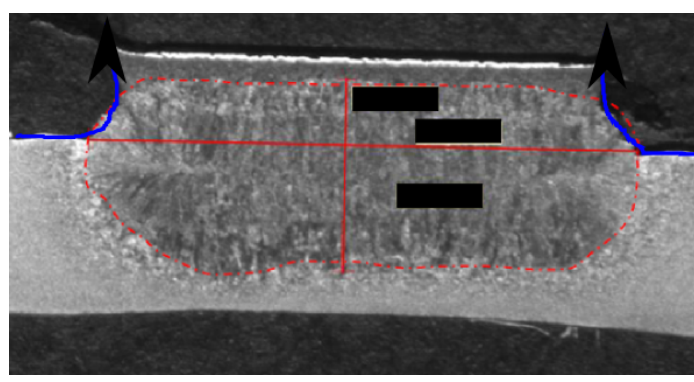

(a) PDF mode. U08D15.

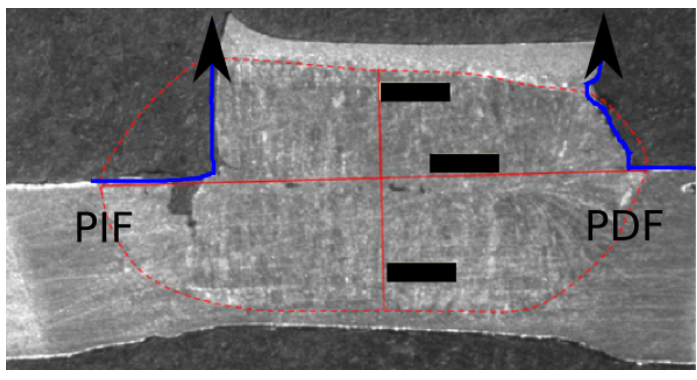

(b) Mix of PDF and PIF modes.

Figure 6.10: Cross-sectioned failure spot welds of dissimilar joints normal tests.

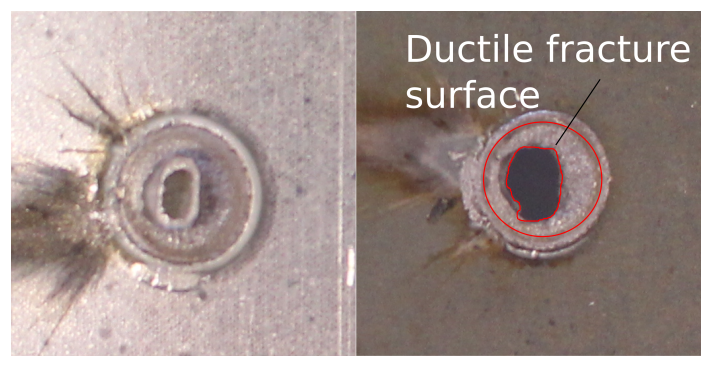

(a) PIF in small diameter joints (U15D15m).

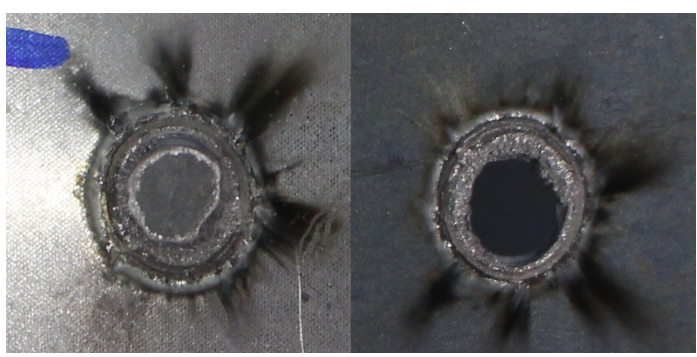

(b) PIF in large diameter joints (U15D15M).

Figure 6.11: Top view of failure spot welds of dissimilar joints normal tests.

In conclusion, despite the fact of the dissimilar appearance of the failed joints, in every case the crack is triggered by the weld notch stress concentration. Consequently, the $J$-integral approach can be also applied in this case. 
6.5. Model validation

\subsection{Model validation}

\subsubsection{Detailed model features}

The same approach presented in the previous chapters for similar joints is applied here for the dissimilar joints spot weld fracture modeling. The only distinction is the differently applied plasticity zones characterization.

Under the modeling point of view, the dissimilar joints spot welds are divided in three main zones, the zone related to the 22MnB5 sheet, the zone related to the DP 980 sheet and the FZ that is a mix of both materials (see Figure 6.12). The zones related to the two joined materials are modeled with the same properties used for the welding zones of the similar joints. However, the strain hardening of the fusion zone is modeled using the mixing rule presented in Equation 6.1. Using the same model set up of previous chapter, FEM simulations are performed. The simulated results are used to calculate the $J$-integral as presented in the previous chapters.

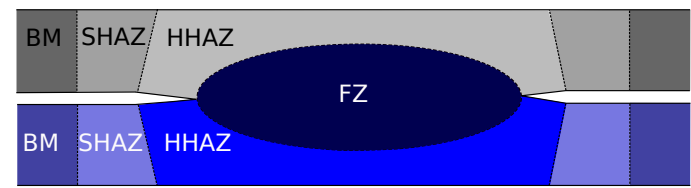

Figure 6.12: Schematic representation of the welding zones of dissimilar joints under plasticity modeling point of view. Grey zones refer to $22 \mathrm{MnB} 5$ material and blue zones are related with the DP 980 material. The mix fusion zone is in the middle.

\subsubsection{Force-displacement curves and maximum forces validation}

Simulated force-displacement curves of shear tests are plotted in Figure 6.13(a). The maximum force of the simulations are from the critical $J$-integral evaluation using the same critical value $\left(J_{I I C}=56 \mathrm{~kJ} \mathrm{~m}^{-2}\right)$ in the same way as the previous chapters. The most representative curves of the two experiments are also plotted and the results of the experiments and simulations correspond with each other.

Similarly, the force-displacement curves for mix-mode tests are presented in Figure 6.13(b), In this case, the same critical value of $J$-integral $\left(\mathrm{J}_{I I C}=56 \mathrm{~kJ} \mathrm{~m}^{-2}\right)$ is the value used for mix-mode and shear tests of every previously analyzed joint of this thesis. The agreement of the curves is low in this case, because of the fact 


\section{Application in Resistance SPOT WELds OF TWO DifFERENT STEEL}

\section{GRADES}

that the boundary conditions of the experimental test cannot be reproduced exactly. However, the same trends followed by the experimental results regarding the loading slopes depending on the thickness combinations can be observed in Figure 6.13(b)

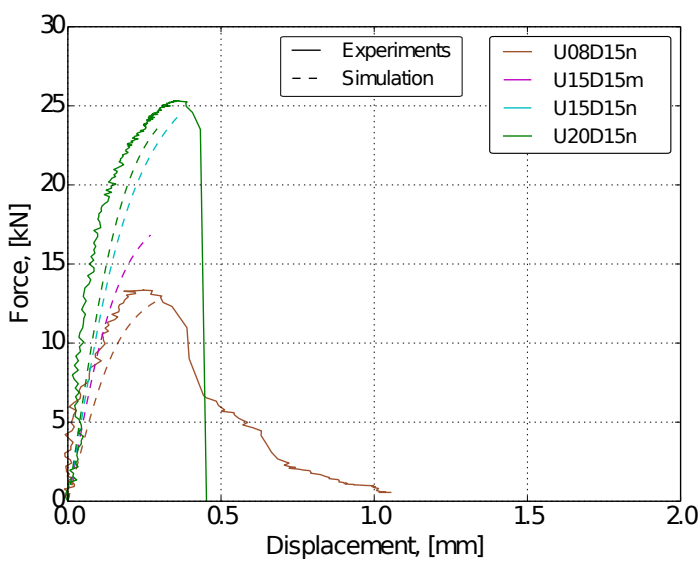

(a) Shear tests.

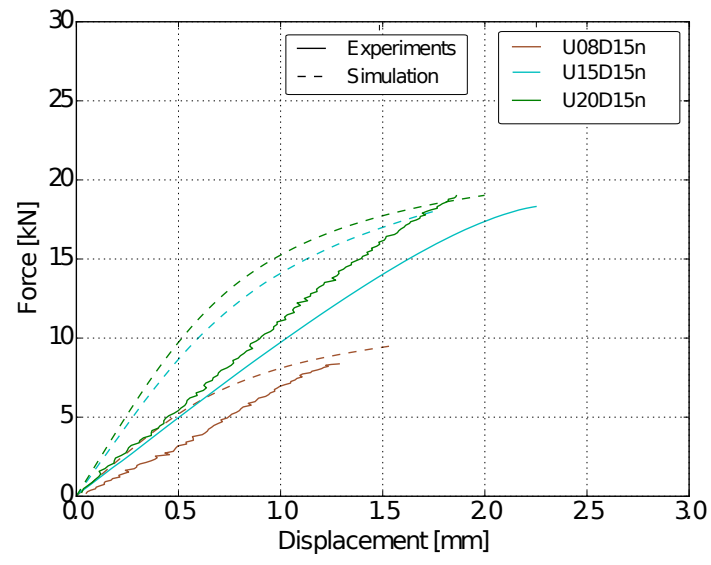

(b) Mix-mode tests.

Figure 6.13: Comparative graph between force-displacement curves of dissimilar joints. Notice that the experimental displacement are those of DIC system, that have been measured in this case at the specimens.

The experimental and simulated force-displacement curves of normal tests are presented in Figure 6.14(a), As in the normal tests presented for similar joints in the previous chapters of this work, the slope of the experimental and simulated curves are not identical due to the unreliable boundary condtions as explained in Figure 4.24. In the same way as with the DP 980 similar joints, if $J_{I C}$ is set to $22.5 \mathrm{~kJ} \mathrm{~m}^{-2}$ as in 22MnB5 similar joints, the maximum forces obtained in Figure 6.14(b) are clearly underestimated. It can be explained with the strong variation of the fracture toughness (i.e. $J_{I C}$ ) of martensitic steels in the transition from $450 \mathrm{HV}$ values of the dissimilar nugget to the $500 \mathrm{HV}$ of the similar 22MnB5 nugget [84].

The maximum forces obtained with the FE model are compared in Figure 6.15 with the experimental maximum forces for all tested combinations. An excellent agreement is achieved in the shear tests for all combinations. There two reasons for the overestimation observed in the U15D15m combinations: the welding expulsions in the failed spot welds and the uncertainty of the measured diameters. In order to clearly identify the influence of the diameter measurement errors the maximum forces are plotted against the weld diameter in Figure 6.16(a), These graphs also consider the uncertainty of the experimental diameter measurements as a factor. The 


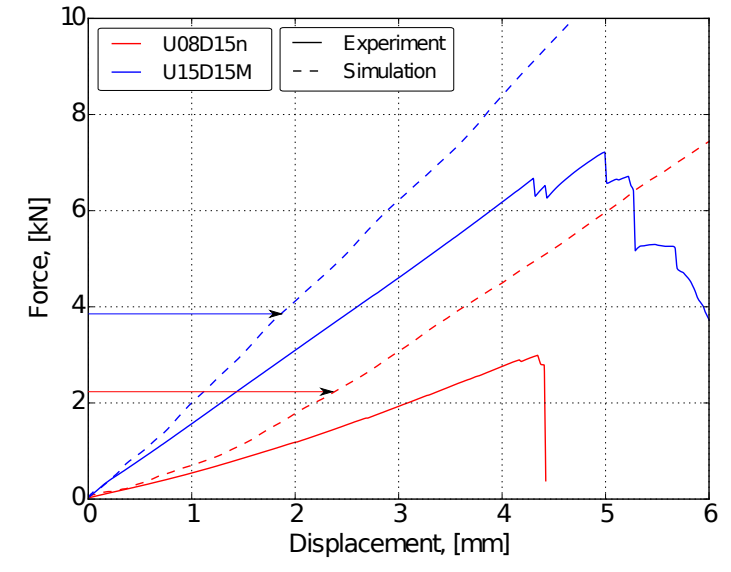

(a) Force-displacement curve.

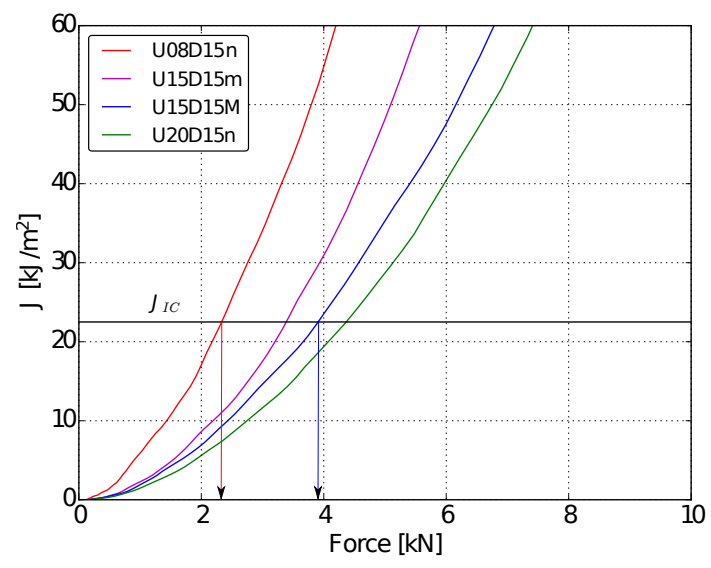

(b) $J$-integral evalution.

Figure 6.14: Experimental and simulated results of normal tests of dissimilar joints.

same conclusion can be applied to the underestimation of U20D15n joint forces. The results of mix-mode tests are presented in Figure 6.15(c). There is a clear agreement between the simulated and experimental results in all cases. The discrepancies in U08D15n and U20D15n are explained by the diameter measurement errors.

For the normal tests forces presented in Figure 6.15(b), if the $J_{I C}=22.5 \mathrm{~kJ} \mathrm{~m}^{-2}$ is used, there is a clear underestimation of the maximum force. For this reason, the experimental critical forces along with the obtained critical forces obtained by simulation using multiple critical $J$-integral values are plotted against the weld diameters in Figure 6.16. A value of $J_{I I C}=56 \mathrm{~kJ} \mathrm{~m}^{-2}$ seems to be the most suitable for maximum force fitting. However, because of the uncertainties in the experimental maximum forces and the set up conditions this value has to be taken with caution. Using this value, the obtained maximum forces are plotted in Figure 6.15(b) as well leading to better prediction. A clear overestimation can be seen in combination U08D15n. This overestimation may be caused by the reduction of bearing capacity of this joint due to the soft zone of the nugget boundary that is not introduced in the FEM model. This soft zone triggers the fracture in these tests at the nugget boundary as presented in Figure 6.10(a), As a quantitative conclusion, notice that because the critical $J$ integral is higher in these joints, the sensitivity of the maximum force to the weld diameter is higher than in the similar 22MnB5 joints because $J$-integral-force curves are more separated when the J-integral values are higher (see Figure 6.14(b)].

Both simulation and experimental maximum forces are plotted and compared 


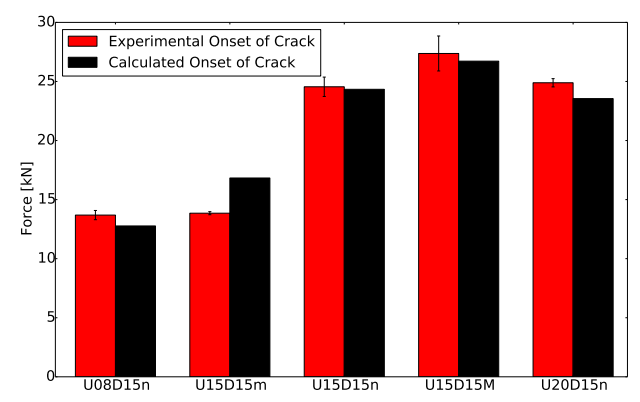

(a) Shear tests.

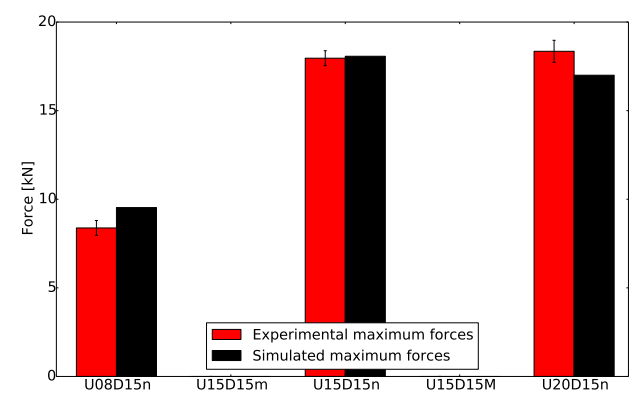

(c) Mix-mode tests.

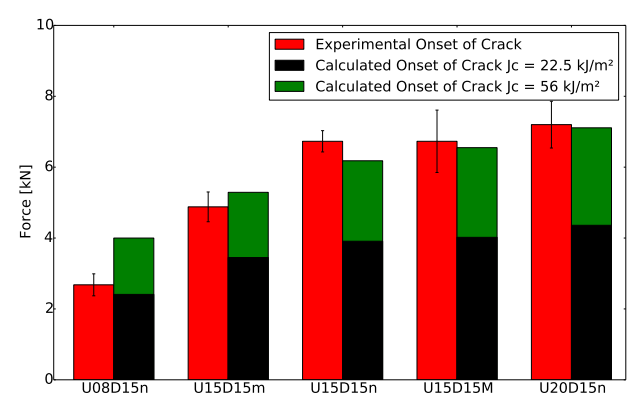

(b) Normal tests.

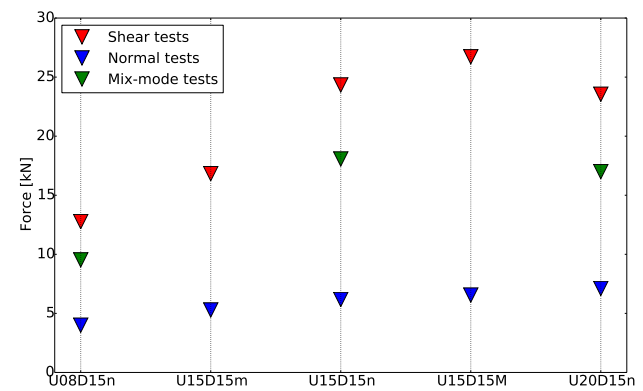

(d) All cases.

Figure 6.15: Comparative graph between calculated and experimental critical forces of dissimilar joints. Experimental results are represented with error bars showing the standard deviation of the five tests performed for each combination.

in Figure 6.15(d) for all loading angles. The detailed FE model can estimate the forces accurately in all cases. It can be seen as well, that with the loading specimen configuration used in this thesis, a joint can bear a higher capacity in shear tests but has a much lower bearing capacity in normal tests. The bearing capacity of mix-mode tests are in between the other two, due to the influence of the phenomenon of both pure loading cases. 


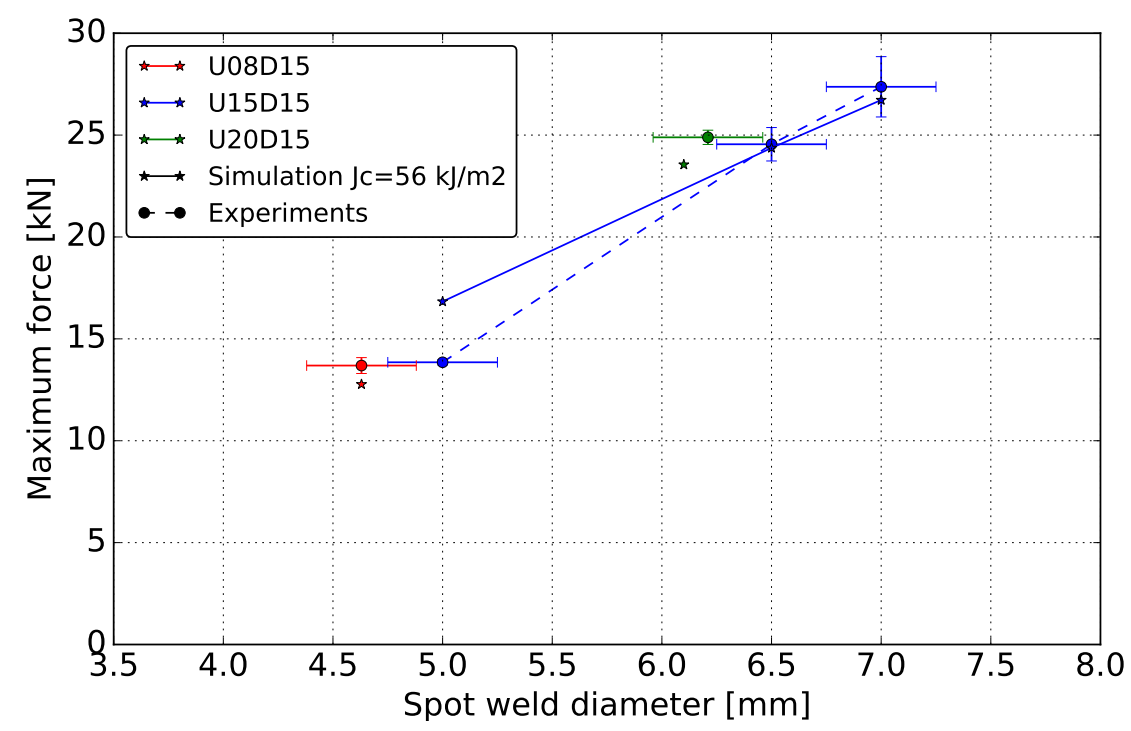

(a) Shear tests.

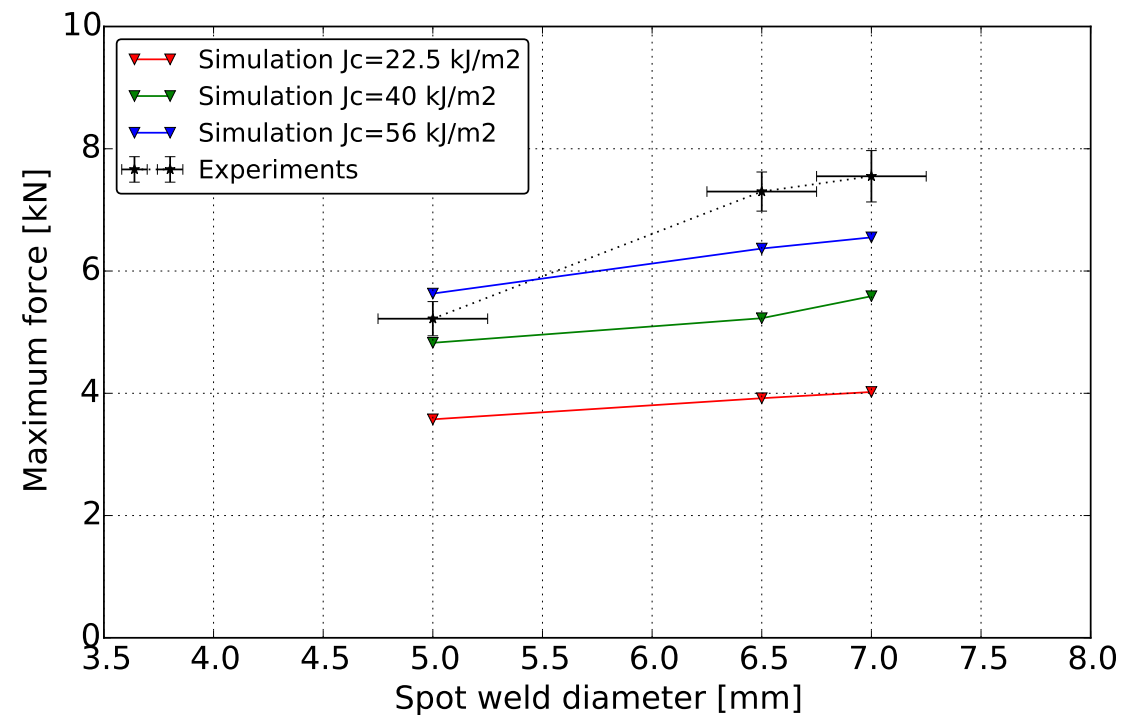

(b) Normal tests.

Figure 6.16: Experimental and simulation forces at multiple diameters in dissimilar 22MnB5 and DP 980 joints. The experimental results are plotted with error bars for the forces representing the standard deviation and error bars of welding diameters considering the measurement errors of $0.2 \pm \mathrm{mm}$. The colors indicate the different thickness combinations. The lineal dependency of the maximum force with the weld diameter appears in the shear tests, it can be seen that a small deviation in the weld diameter of the FE model would produce significant differences in the maximum force, $0.1 \mathrm{~mm}$ error leads to $0.5 \mathrm{kN}$ of maximum force difference. 


\subsubsection{Influence in the results of unaccurte nugget plasticity characterization}

The results presented in Section 6.5.2 were acquired using the mix-rule introduced in Equation 6.1 for the characterization of the nugget plasticity model, that is interpolated from the similar joints nugget of the two welded materials. However, the hardening curve of this zone obtained with this method diverges at large strains from the curve obtained from the inverse FEM optimization procedure using micro welded specimens with mix nugget material in the gauge zone as presented in Figure 6.5(b).

In order to analyze the robustness of the acquired results witch depend on the nugget material plasticity characterization, the simulated results of the force-displacement curves and $J$-integral evolution obtained with the two possible approaches are presented in Figures 6.17 and 6.18. In addition, the results of both tests appear to be identical. These results can be explained by the large strains during weld loading are confined to a small region close to the weld notch, therefore differences in the characterization of this zone would not produce significant differences in the final results.

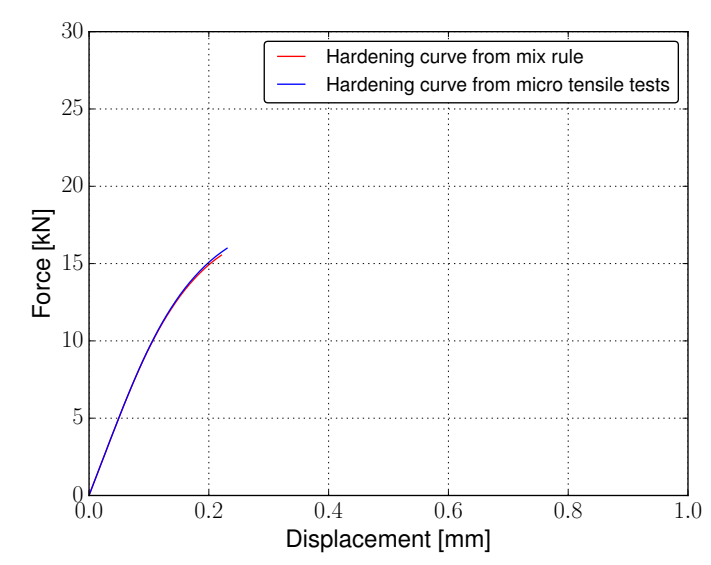

(a) Force-displacement curve.

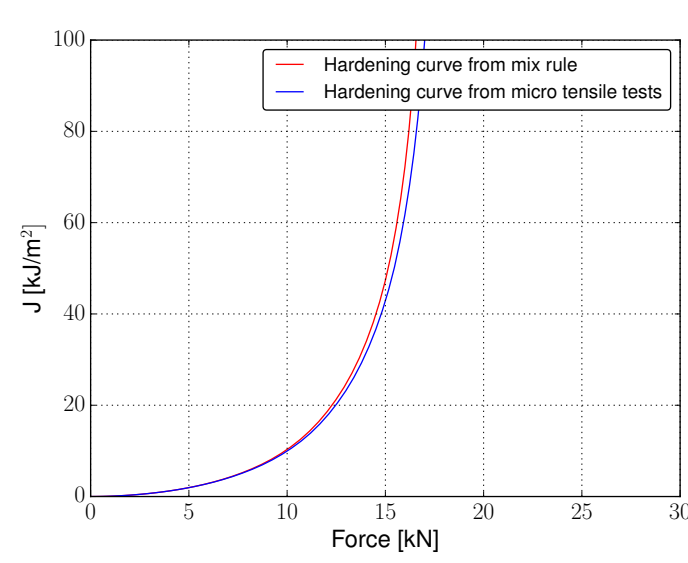

(b) $J$-integral evolution.

Figure 6.17: Simulation results of dissimilar shear tests depending on the method of obtaining the nugget hardening curve.

Another important conclusion determined by these results is the strong stability of the maximum force results obtained using the $J$-integral as fracture criterion independent of the inaccuracy of the welding zones material characterization. 


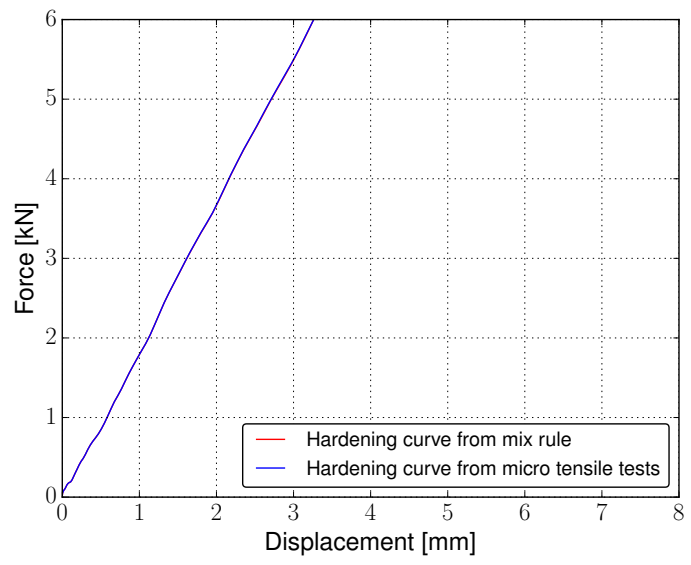

(a) Force-displacement curve.

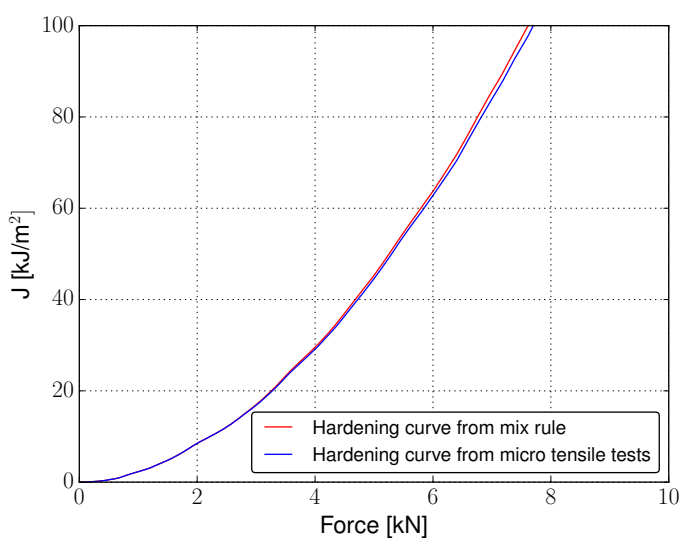

(b) $J$-integral evolution.

Figure 6.18: Simulation results of dissimilar normal tests depending on the method of obtaining the nugget hardening curve. 



\section{Chapter 7}

\section{Model application in real vehicle crash simulations}

In the previous chapters, a detailed FEM model for predicting the bearing capacity of RSWjoints in AHSS sheets under out-of-plane loading conditions has been developed. The failure model has been characterized and validated for joints involving sheets of two different steel grades, a hot formed steel and a cold formed steel. Very promising results have been obtained for all welded configurations taking into account that were manufactured and tested with controlled laboratory conditions.

However, the presented model is still far from useful for full-vehicle crash simulations because of two main reasons. On the one side, the high computational costs of the detailed spot weld model are unaffordable for the full-vehicle crash simulations with thousands of spot welds. On the other side, the model has been validated only for a reduce amount of configurations and loading combinations, nevertheless in a vehicle crash event thousands of spot welds of multiple configurations are loaded under complex conditions.

This chapter discusses the differences between the spot weld simulated results previously analyzed and the real spot weld response during a vehicle crash situation. First, in Section 7.1 there is an analysis of sheets' combinations that can be found in vehicles structure and have not been considered in the previous chapters. The complex loading conditions of joints during crash situations are analyzed in Section 7.2 . Finally, in Section 7.3 the geometry of the spot welds welding under laboratory con- 
ditions is compared with the actual spot welds shapes that can be found in a vehicle $\mathrm{BiW}$ of the production line.

\subsection{Steel sheet combinations in vehicle structure}

Only joints of two sheets of two different AHSS grades have been analyzed in this thesis. However, in modern vehicle structure, there are spot welds involving multiple steel grades as well as joints of more than two sheets. The most repeated welded combinations in a vehicle structure that have not analyzed previously are presented here, i.e. those involving ductile steels, those joining other AHSS grades and joints of three sheets.

As presented in the Chapter 3 of this thesis, the spot welds that join a ductile steel are prone to fail due to necking in the base material surrounding the joint with a much lower mechanical properties than the weld nugget material (see Figure 2.5). Therefore, the $J$-integral-based approach is not applicable in these cases. Nevertheless, failure would be predicted in the full-vehicle models by the strain fracture criterion applied to the surrounding sheets of the joints modeled by shell elements in the full-vehicle models.

Furthermore, to analyze welds of a different AHSS grade to those evaluated previously in this thesis the characterization procedure presented in Chapters 4 and 5 is proposed:

1. A detailed analysis of the new material spot welds in order to identified the main welding zones and to know which ones should be characterized.

2. A characterization of the strain hardening curves of the main zones using microtensile tests or hardness-based characterization. Notice that a precise characterization of these zones is not required.

3. A model validation using experimental normal and shear loading tests and the characterized detailed FEM model. In order to obtain the critical $J$-integrals both for mode I and mode II, independent characterization tests (such as the standardized fracture mechanics test specimens [44]) are recommended. It should be noticed that these specimens must be manufactured in such a way that the crack propagation during testing occurs in the weld fusion zone. The independent tests for $J_{I I C}$ characterization are specially recommended for the 
normal tests due to the strong non linearity of the critical value depending on the nugget composition and subsequent hardness.

In the case of dissimilar joints that weld a AHSS grade different from those already presented in this thesis, the procedure from Chapter 6 is recommended, i.e.:

1. A characterization of HAZ and FZ using data extracted from similar joints

2. Obtaining the strain-stress curves of the mixed weld nugget using the rule of mixtures based on the hardening curves extracted from the similar joints.

Finally, the joints at which three sheets are connected can be treated as two separate spot welds under the modeling point of view. Usually in a real vehicle BiW, the more repeated combination regarding AHSS sheets is a joint of two high strength sheets with a ductile steel thinner sheet at the external part (see Figure 7.1). In these cases, during real vehicle crash situations it is experimentally observed that the structure welded joints failure occurs mainly because of the necking of the surrounding ductile steel material. This failure would be detected by the sheet fracture-strain criterion in full-vehicle simulation. However, in order to predict the bearing capacity of the joint at the two AHSS sheets side, a $J$-integral approach should be applied in the corresponding notch. Theoretically, the same aforementioned procedure for a new material should be followed, i.e. characterization of the similar joint nugget material of each one of the three steels and the use of the rule of mixtures in order to obtain the plasticity of the mixed nugget. Nevertheless, as a first approximation the calculation of the bearing capacity of the two AHSS sheets joint can be treated as an isolated joint. It must be treated as a joint with the corresponding diameter and the properties distributions of the spot weld without taking into account the influence of the ductile steel.

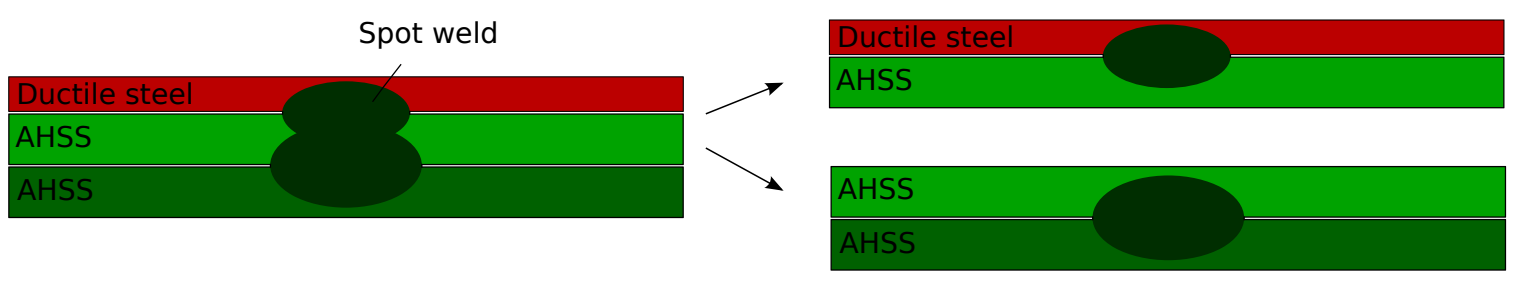

Figure 7.1: Scheme of cross sectioned spot weld joining three sheets. These joints can be modeled as two separate joints. 


\subsection{Loading conditions during crash situations}

The detailed spot weld model was validated by applying three different loading angles to the joints in order to test the fracture response. However, in a real vehicle crash situation the structure joints are loaded at complex loading conditions. For this reason, isolated welds are usually experimental tested at different angles (e.g. using KS-II at $60^{\circ}$ and $30^{\circ}$ ). However, it must be taken into account that the total applied displacement can be divided in normal and tangential components and normal direction has much lower resistance due to the material bending (see Section 3.8). Consequently, the applied displacement in $30^{\circ}$ and $60^{\circ}$ directions lead to a strong resistance related with the tangential component. This higher resistance in tangential direction leads to predominant mode II failure in these tests. Consequently, to simulate these mix-mode tests using the present detailed model, $J_{I I C}$ is recommended unless pure normal displacements are applied. On the contrary, for the peeling tests and normal tests, there is no tangential component applied to the weld, therefore the mode $\mathrm{I}$ is dominant and $J_{I C}$ is recommended.

\subsection{Differences between idealized and actual spot welds}

The joints presented in this work have been welded in ideal laboratory conditions and those joints with clear visible imperfections have been rejected to validate the model. However, in an actual automobile structure, imperfections and irregularities can frequently be seen in the resistance spot welds. Some of these irregularities are permitted by the welding norm if their dimensions are under some limited values for quality level. Some of these imperfections are micro-cracks in the nugget or in the heat affected zone, pores in the nugget, porosity, metallic or oxidic inclusions, lack of fusion, welding splashes leading to pressed out material, asymmetric nuggets, and nugget penetration depth too small or excessive gap between sheets. Figure 7.2 shows a scheme of some of the imperfections accepted by the norm.

All these imperfections lead to the fact that geometry of an actual spot weld in a car structure may differ for the idealized geometries of the weld used in this thesis. For example, an irregular geometry of a spot weld from a real car structure can be seen in Figure 7.3. Likewise, some imperfections in the base material mechanical 
7.3. Differences between idealized and actual spot welds

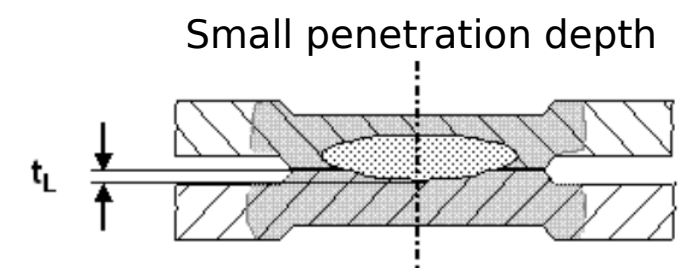

Assymetric nugget
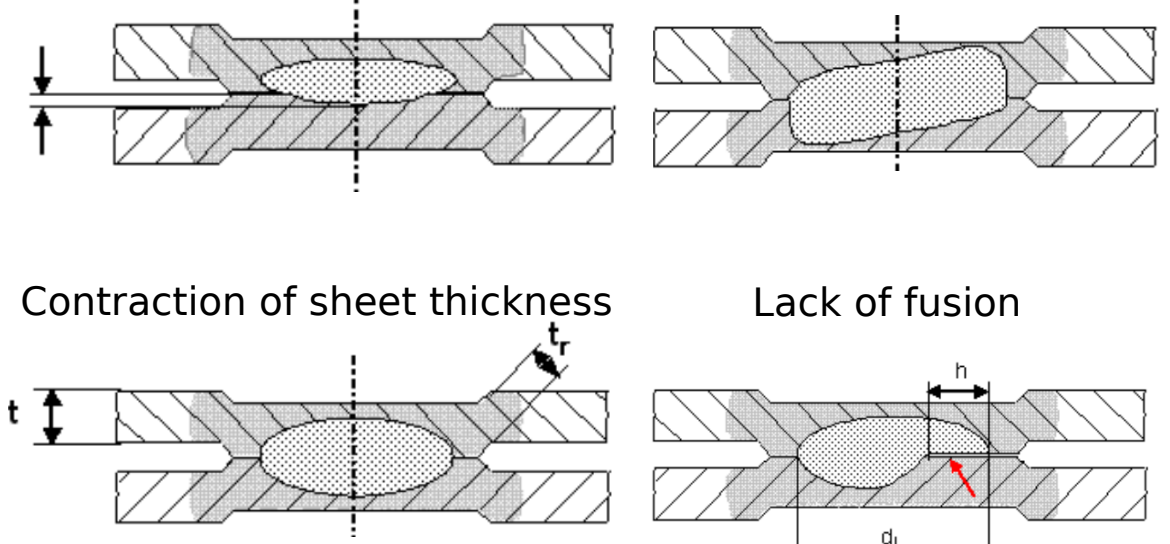

\section{Lack of fusion}

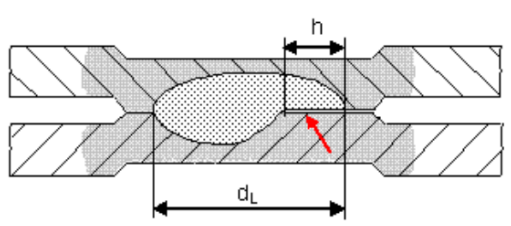

Figure 7.2: Sketch of some welding irregularities permitted by the welding norm [1].

properties are permitted in the norm for the structural sheets, particularly in the case of hot formed steels. As an example, hardness values of less than $450 \mathrm{HV}$ are permitted for some zones of the sheets due to the stamping conditions.

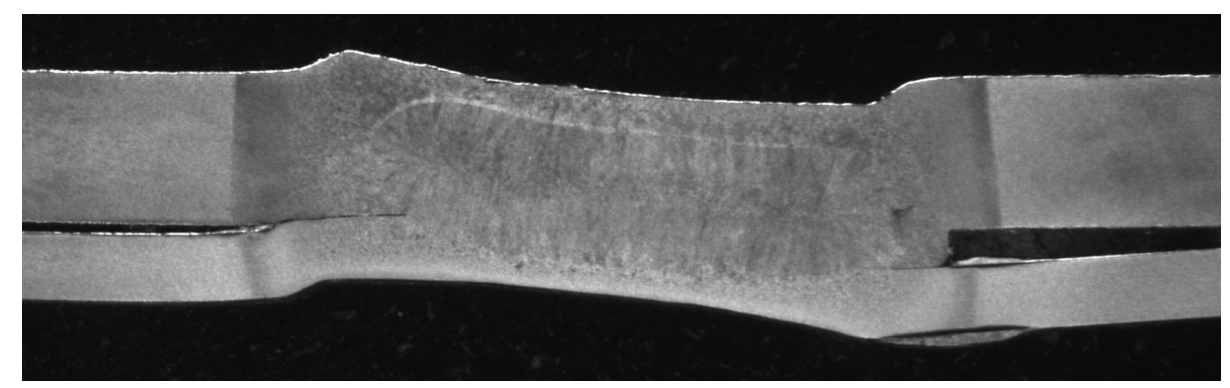

Figure 7.3: Cross section of a spot weld extracted from a car body of the production line. The irregular geometry is caused by electrode misalignment during welding.

All these imperfections may reduce the bearing capacity of joints when comparing them with the results of the detailed finite element model presented in this thesis. The quantification of this reduction of the bearing forces is extremely complex and must be done using an stochastic analysis. This analysis is beyond the scope of the present thesis. $\mathrm{r}$ 



\section{Chapter 8}

\section{Conclusions}

Current full-vehicle crash simulations rely on very simple elastic beam models for resistance spot welds. In the case of AHSS, failure of the joints is critical. The spot welds are assumed to fail when a certain bearing capacity is reached. A very extensive and expensive campaign is needed in order to characterize the bearing capacity of the joints depending on the geometry of the weld (and thus welding conditions), the loading angles, the thickness and steel grade of the AHSS sheets, among other parameters. The main objective of this thesis is to alleviate the cost of the experimental characterization campaign by developing a detailed finite element model to reproduce the experimental characterization tests of spot welds in AHSS and determine the bearing capacity of the joint with enough level of accuracy. This bearing capacity is the only joint failure parameter that is fed to the full-vehicle crash simulations regarding the thousands of joints present in a vehicle. The model has been validated against experiments for multiple possible combinations of sheets' thicknesses, loading conditions and material grades based on the automobile crashrelevant structural components.

Based on preliminary results of a spot weld model it was concluded that it is necessary to have a detailed description of the weld geometry using a fine mesh, to introduce the right material mechanical characterization of the welding zones and an adequate fracture criterion. The bearing capacity of the joints has been estimated in the simulations of this thesis using an energetic $J$-integral-based fracture criterion. This criterion has the advantage of being extremely robust, not too sensitive to the unavoidable small variability in the geometry and the material properties of the welds. 
In addition, it is simple to implement and to use. The model returns curves of $J$ integral values versus force. By simply intersecting these curves with a critical value (a mechanical parameter for the joint material) an estimate for the bearing capacity of the joint is obtained, the critical value of the J-integral depends on the fracture mode.

The model has been applied to joints in the hot formed martensitic boron steel fully hardened 22MnB5, then to joints in the cold formed dual phase steel DP980 and finally to joints between both steel grades. The procedure followed in all cases is to compare the results of the detailed finite element model with those obtained with the experimental loading tests. On one side, the corresponding hardening curves of the welding zones are applied to the spot weld model and with the FEM simulation results, the $J$-integral is evaluated to obtain the maximum forces. On the other side, the joints are experimentally tested using different configurations and the experimental maximum forces are obtained.

In the case of fully hardened $22 \mathrm{MnB} 5$ spot welds, the calibration of the hardening curves of the different welding zones obtained from a previous work [18] has been used for the model. Furthermore, micro-tensile tests have been peformed in order to check that the plasticity of the martensitic nugget is the same as the fully hardened 22MnB5. Multiple experimental loading tests have been carried out to validate this model, considering different sheets' thickness combinations, spot weld sizes and loading modes. From these experiments, important conclusions have been extracted. The fracture begins in all cases from the weld notch. However, two fracture modes with corresponding spot weld fracture surfaces have been distinguished. On one side, ductile fracture curves and surfaces have been observed in the joints loaded in the normal tests where mode I failure occurs. On the other side, brittle shear fracture and associated brittle force-displacement curves are observed in both shear and mix-mode tests, which fail under mode II. Two critical $J$-integral values extracted from previous works are used in the simulations in order to obtain the critical forces, one for mode I (i.e. $J_{I C}$ and a different one for mode II (i.e. $J_{I I C}$ [5, 6, 10, 17]. The experimental and simulated results exhibit good agreement in almost all analyzed cases using the proposed model. The observed discrepancies are mainly attributed to imperfections and mechanical phenomena in the actual welds that are very difficult to model, such as welding defects, voids or a extremely localized soft zone around the fusion zone.

For the validation of the model for DP 980 joints, a similar procedure has been 
applied. Several experimental loading tests of spot welds of different sizes and loaded at different angles have been performed in this case as well. In these experimental tests, the same failure modes of those of $22 \mathrm{MnB} 5$ joints have been observed. Small differences have been detected when comparing with $22 \mathrm{MnB} 5$ joints: On one side, a stable crack advance after maximum force is observed for the shear tests; on the other side the kink of crack propagation direction for some normal tests leads to different final failed spot weld appearance. Regarding the detailed spot weld model, the hardening curves of the martensitic weld nugget were not available. These curves have been obtained in this thesis from micro-tensile tests of welding specimens using an inverse FEM optimization procedure. These hardening curves, along with those of the base material already available, have been introduced in the spot weld model to perform the simulations where the $J$-integral is evaluated. The obtained maximum forces present very good agreement with the experiments for shear and mix-mode tests when using the same $J_{I I C}$ value as in the $22 \mathrm{MnB} 5$ joints. However, when $J_{I C}$ value of $22 \mathrm{MnB} 5$ joints is used for normal tests of DP 980 steel joints, the maximum forces are underestimated. A higher $\overline{J_{I C}}$ value has been proposed, in accordance with previous works where this value depends on the hardness value of a martensitic steel. These differences in the hardness values have been observed between the nuggets of 22MnB5 and DP 980 welded joints due to the different carbon contain of both steels.

Lastly, the model has been validated for dissimilar joints, i.e. joint where sheets of both steels are involved. In this case, the experimental loading tests campaign include different sheets' thickness combinations, spot weld sizes and loading angles. The same fracture modes observed in similar joints have been detected in this case. Furthermore, regarding the detailed model apart from the already available plasticity parameters of both BMs and HAZ, the model should be completed with the hardening curves of the homogeneous mixed fusion zone, where neither data nor a procedure to obtain them were available. Consequently two methods have been proposed to obtain the hardening curves: to apply the rule of mixtures using the hardening curves of the nugget of both 22MnB5 and DP 980 joints and to perform micro-tensile tests along with the FEM optimization procedure (as introduced for DP 980 weld material). Both approaches presented similar results and the rule of mixture is recommended for future works because of the simplicity. The maximum forces obtained with the spot weld model using these curves exhibit good agreement with the experimental results for shear and mix-mode tests using the same $J_{I I C}$ of $22 \mathrm{MnB} 5$ and DP 980 
similar joints. However, for the same reason as in the DP 980 joints (the martensitic nugget carbon contain), a higher $J_{I C}$ than for $22 \mathrm{MnB} 5$ joints is proposed to reproduce correctly the maximum forces of normal tests.

The extracted conclusions have been analyzed for other joints configurations that can be found in a full-vehicle structure. The obtained information about spot weld failure in joints of AHSS with the present work will be very useful in the future for a better vehicle structure development. The most important conclusions are the strong sensitiveness of the joints to the weld size under shear loading conditions, the reason of the lower bearing capacity of the joints when the weld notch is loaded in normal direction and the reduction of spot welded joints maximum forces in the case of weld imperfections such as voids or splashes. 


\section{Appendix A}

\section{Implementation of the $J$-integral calculation in the FE framework}

As presented in Chapter 3 of this thesis, the detailed modeling of resistance spot weld fracture must be done using elastic-plastic material models using a criterion to asses the stress concentration around the weld notch. The contour $J$-integral introduced by Rice is usually used for the characterization of fracture in theses cases [78]. The $J$-integral was initially presented for a two-dimensional, planar, cracked nonlinear elastic body, using the small-strains assumption and neglecting internal body forces. The Rice $J$-integral is given by

$$
J_{1}=\int_{\Gamma}\left[W n_{1}-\sigma_{i j} \frac{\partial u_{i}}{\partial x_{1}} n_{j}\right] \mathrm{d} S
$$

Hereby $\Gamma$ designates an arbitrary counterclockwise path drawn from the lower to the upper crack flank (see Fig. A.1); $\boldsymbol{n}$ is the outward normal unit vector to $\Gamma, W$ is the strain energy density, $\boldsymbol{\sigma}$ the Cauchy stress tensor, $\boldsymbol{u}$ the displacement, and $\mathrm{d} S$ the arc length increment along $\Gamma$. The subindex 1 in $J$ refers to the evaluation of the $J$-integral in the $x_{1}$ direction. 


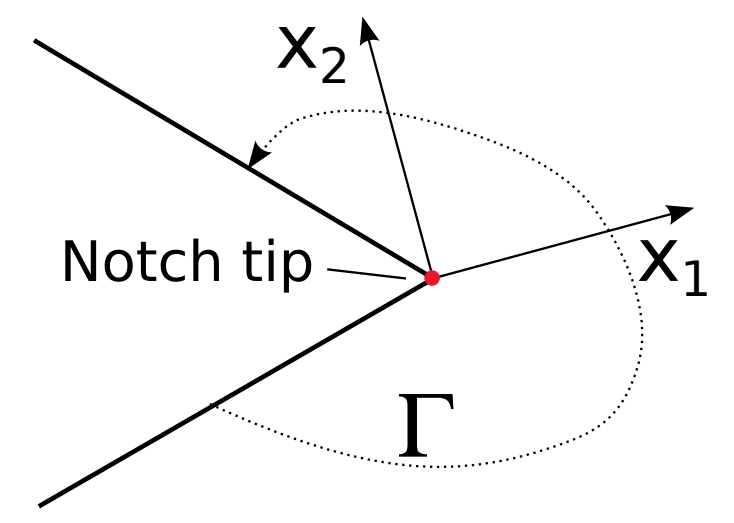

Figure A.1: Crack front and $J$-integral coordinate system and arbitrary contour.

\section{A.1 Evaluation of $J_{1}$ using the equivalent domain integral technique}

In the present thesis, the $J$-integral is calculated from the results of the stress, strain and displacement fields obtained from FE calculations. The form of the $J$-integral in Eq. A.1 is poorly suited to numerical analysis. For this reason, it was expressed in terms of an area integral and converted into a volume integral following the equivalent domain integral (EDI) technique [2, 94, 95]. In addition, the deformation theory of plasticity, as exposed by Anderson [44], can be applied under the conditions of proportional loading. Considering all these statements, the $J$-integral is evaluated in a three dimensional cracked body after applying the divergence theorem in Eq. A.1 and introducing an arbitrarius but continuous function (it is known as $s$-function and is explained in Section A.1.3. Therefore $J$-integral is evaluated following the next equation:

$$
J_{1}=\frac{J_{S}+J_{W}+J_{A}}{f}
$$

where $f$ is evaluated from $s$-function. The different terms are defined by

$$
\begin{gathered}
J_{S}=-\int_{V-V_{\epsilon}}\left(W \frac{\partial s}{\partial x_{1}}-\sigma_{i j} \frac{\partial u_{i}}{\partial x_{1}} \frac{\partial s}{\partial x_{j}}\right) \mathrm{d} V, \\
J_{W}=-\int_{V-V_{\epsilon}}\left[\frac{\partial W}{\partial x_{1}}-\frac{\partial}{\partial x_{j}}\left(\sigma_{i j} \frac{\partial u_{i}}{\partial x_{1}}\right)\right] s \mathrm{~d} V,
\end{gathered}
$$




$$
J_{A}=\int_{A_{1}+A_{2}}\left(W n_{1}-\sigma_{i j} \frac{\partial u_{i}}{\partial x_{1}} n_{j}\right) s \mathrm{~d} A
$$

$V_{\epsilon}$ and $V$ refer to cylindric domains surrounding the crack front. $V_{\epsilon}$ and $V$ have both the same axys that is tangent to the crack front line. $V_{\epsilon}$ is defined in order to avoid computing terms in the vicinity of crack front due to the numerical issues of FEM solution related with the notch tip singularity. $A_{1}$ and $A_{2}$ are the both lateral surfaces of a domain defined as $V-V_{\epsilon}$ as presented in Fig. A.2. The different terms are evaluated in the present work as follows.

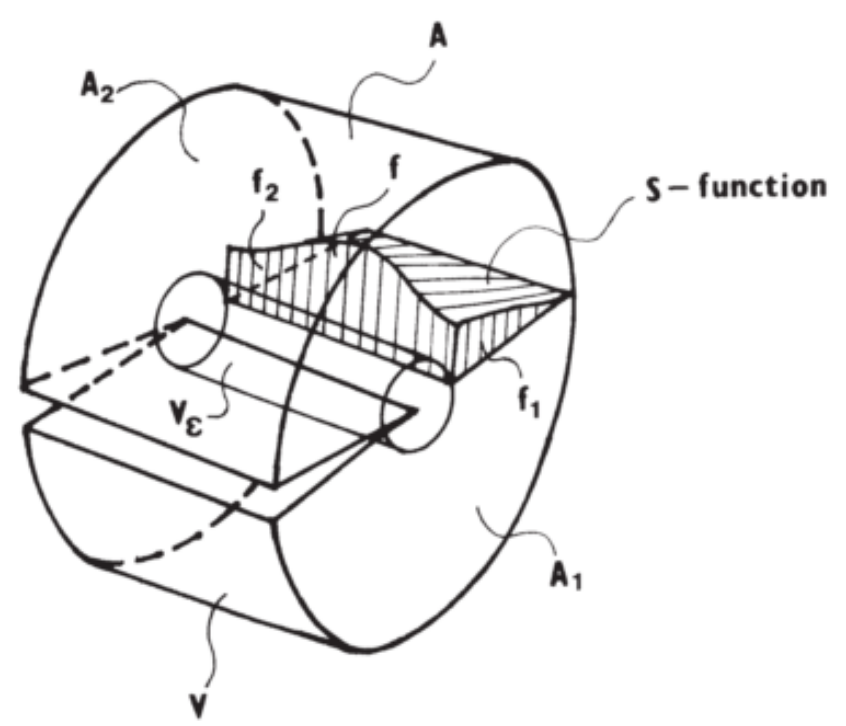

Figure A.2: Integral 3D domain around the notch tip. Surfaces, volume and $s$ function definition. Taken from [2].

\section{A.1.1 Evaluation of $J_{S}$ using finite element values with one integration point}

$V-V_{\epsilon}$ is a volumetric domain that can be subdivided into a finite number of finite elements, therefore Eq. A.3 can be written as

$$
J_{S}=-\sum_{\text {elements }}\left\{J S_{\text {element }}\right\}
$$




\section{A. Implementation of the $J$-InTEGRAL CALCUlation in the FE} FRAMEWORK

Considering, the parametric representation of displacements and $s$-function

$$
u_{i}=N^{M} u_{i}^{M} \quad s=N^{L} s^{L}
$$

where $N^{M}$ are the 'shape' functions, $i$ is the direction of the crack-front coordinate system and the superscripts $M$ and $L$ refer to the node number. And taking into account the rules for volume integration in the finite element framework, Eq. A.3 can be written for each element as

$$
J S_{\text {element }}=-\int_{-1}^{+1} \int_{-1}^{+1} \int_{-1}^{+1}\left\{W \frac{\partial N^{L}}{\partial x_{1}} S^{L}-\sigma_{i j} \frac{\partial N^{M}}{\partial x_{1}} \frac{\partial N^{L}}{\partial x_{j}} u_{i}^{M} S^{L}\right\}(\operatorname{det}(J)) \mathrm{d} V
$$

where $\operatorname{det}(J)$ is the determinant of the Jacobi matrix. This equation can be written as

$$
J S_{\text {element }}=-R^{L} S^{L}
$$

where $S^{L}$ is the value of $s$-function at each node and $R^{L}$ is defined by

$$
R^{L}=\int_{-1}^{+1} \int_{-1}^{+1} \int_{-1}^{+1}\left\{W \frac{\partial N^{L}}{\partial x_{1}}-\sigma_{i j} \frac{\partial N^{M}}{\partial x_{1}} \frac{\partial N^{L}}{\partial x_{j}} u_{i}^{M}\right\}(\operatorname{det}(J)) \mathrm{d} V .
$$

Using the quadrature rule of finite elements implemented in the used VPS - MMM code, the Eq. A.10 can be written as

$$
R^{L}=\left\{W \frac{\partial N^{L}}{\partial x_{1}}-\sigma_{i j} \frac{\partial N^{M}}{\partial x_{1}} \frac{\partial N^{L}}{\partial x_{j}} u_{i}^{M}\right\}(\operatorname{det}(J)) w_{p}
$$

where $w_{p}$ is the weight corresponding to the Gaussian integration point at a point $\mathrm{p}$.

\section{A.1.2 Total strain energy density}

In the evaluation of $J$-integral following the deformation theory of plasticity, the real elastic-plastic material is treated as nonlinear elastic. Therefore, the total strains are considered elastic. Therefore:

$$
W=W^{e}=\int_{0}^{\varepsilon^{e}} \boldsymbol{\sigma}: d \varepsilon^{e}=\int_{0}^{\varepsilon} \boldsymbol{\sigma}: d \boldsymbol{\varepsilon}=\int_{0}^{t} \boldsymbol{\sigma}: \frac{d \boldsymbol{\varepsilon}}{d t} \mathrm{~d} t .
$$

A time discretization is applied to Eq. A.12 to obtain: 


$$
W_{n}=W_{n}^{e}=\sum_{i=1}^{n}\left[\frac{\boldsymbol{\sigma}_{i}+\boldsymbol{\sigma}_{i-1}}{2}\right]:\left[\varepsilon_{i}-\varepsilon_{i-1}\right]=W_{n-1}+\left[\frac{\boldsymbol{\sigma}_{n}+\boldsymbol{\sigma}_{n-1}}{2}\right]:\left[\varepsilon_{n}-\varepsilon_{n-1}\right]
$$

where $\boldsymbol{\sigma}$ is the Cauchy stress tensor, $\varepsilon^{e}$ is the total strain tensor (asumming that all strains are pure nonlinear elastic) and $n$ indicates the step.

\section{A.1.3 s-function}

The $s$-function is a mathematical artifact to evaluate the $J$-integral in a 3D domain. It must be arbitrary but continuous in the computational domain, and it should have a zero value in the A surface (external surface of domain $V$ (see Fig. A.2) and a non-zero value in $A_{\epsilon}$ (that is the cylindrical surface of $V_{\epsilon}$, the volume of a small tube around the crack front segment).

The $s$-function is defined in such a way that its value is 1 at the inner cylinder $\left(r=\sqrt{\left(x_{1}^{2}+x_{2}^{2}\right)}=r_{\text {min }}\right.$ where $r_{\text {min }}$ is the radius of $\left.A_{\epsilon}\right)$ and 0 at the outer cylinder $\left(r=r_{\max }\right.$ where $r_{\max }$ is the radius of $A$ ) and at both sides of the integral domain $\left(A_{1}\right.$ and $\left.A_{2}\right)$. The defined function will be linear in $r$ and $x_{3}$ direction independently, therefore:

$$
s\left(r, x_{3}\right)=s(r) s\left(x_{3}\right)
$$

where:

$$
s(r)=\left\{\begin{array}{lcc}
1 & \text { if } & r \leq r_{\min } \\
\frac{r_{\max }-t o l S-r}{r_{\max }-r_{\min }-2 t o l S} & \text { if } & r_{\min }<x<r_{\max } \\
0 & \text { if } & r \geq r_{\max },
\end{array}\right.
$$

where tol $S$ is a tolerance value to ensure that at least some nodes of $A_{\epsilon}$ have $s=1$. It depends on the mesh size, for the $0.1 \mathrm{~mm}$ mesh used in this work, the value of tol $S=0.2$ is recommended.

$$
s\left(x_{3}\right)=\left\{\begin{array}{lcl}
\frac{\frac{D L}{2}-\left|x_{3}\right|}{\frac{D L}{2}} & \text { if } & \left|x_{3}\right| \leq \frac{D L}{2} \\
0 & \text { otherwise } &
\end{array}\right.
$$

where $D L$ is the high of the cylinders in Fig. A.2. 
A. Implementation of the $J$-integral CAlCUlation in the FE FRAMEWORK

\section{A.2 Evaluation of the second integral of Eq. A.2,}

$$
J_{W}
$$

It is possible to demonstrate that the second term of $J_{1}$ is zero 93 following the next procedure. Consider the next equality:

$$
\frac{\partial W}{\partial x_{1}}=\frac{\partial W}{\partial \varepsilon_{i j}} \frac{\partial \varepsilon_{i j}}{\partial x_{1}} .
$$

IF deformation plasticity is assumed, then the total strain energy density is recoverable, and consequently stresses have a potential, the components of the stress tensor can be written as

$$
\frac{\partial W}{\partial \varepsilon_{i j}}=\sigma_{i j}
$$

In addition, the momentum equation shown in Eq. B.2 leads to:

$$
\frac{\partial \sigma_{i j}}{\partial x_{j}}=0
$$

and thus, taking Eq. A.19 the next development can be done

$$
\frac{\partial}{\partial x_{j}}\left(\sigma_{i j} \frac{\partial u_{i}}{\partial x_{1}}\right)=\frac{\partial \sigma_{i j}}{\partial x_{j}} \frac{\partial u_{i}}{\partial x_{1}}+\sigma_{i j} \frac{\partial^{2} u_{i}}{\partial x_{1} \partial x_{j}}=\sigma_{i j} \frac{\partial^{2} u_{i}}{\partial x_{1} \partial x_{j}} .
$$

Furthermore, assuming small strains theory

$$
\frac{\partial^{2} u_{i}}{\partial x_{1} \partial x_{j}}=\frac{\partial \varepsilon_{i j}}{\partial x_{1}}
$$

Using all the previous equations it is obtained

$$
\frac{\partial W}{\partial x_{1}}-\frac{\partial}{\partial x_{j}}\left(\sigma_{i j} \frac{\partial u_{i}}{\partial x_{1}}\right)=\frac{\partial W}{\partial \varepsilon_{i j}} \frac{\partial \varepsilon_{i j}}{\partial x_{1}}-\sigma_{i j} \frac{\partial^{2} u_{i}}{\partial x_{1} \partial x_{j}}=\sigma_{i j} \frac{\partial \varepsilon_{i j}}{\partial x_{1}}-\sigma_{i j} \frac{\partial \varepsilon_{i j}}{\partial x_{1}}=0 .
$$

Leading to the conclusion that second term of $J_{1}$ in Eq. A.2 is zero.

\section{A.3 Evaluation of the third integral of Eq. A.2,}

$$
J_{A}
$$

In Eq. A.14 $s$-funtion has been defined in such a way that its value is zero on both sides of the domain $\left(A_{1}\right.$ and $\left.A_{2}\right)$. If this $s$-function is used and applied to Eq. A.5. 
A.3. Evaluation of the third integral of Eq. A.2, $J_{A}$

the value of $s$ is zero in the integration surface domain. Consequently, with the right choice of $s$-function, this third term is also zero.

$$
J_{A}=\int_{A_{1}+A_{2}}\left(W n_{1}-\sigma_{i j} \frac{\partial u_{i}}{\partial x_{1}} n_{j}\right) s \mathrm{~d} A=\int_{A_{1}+A_{2}}\left(W n_{1}-\sigma_{i j} \frac{\partial u_{i}}{\partial x_{1}} n_{j}\right) 0 \mathrm{~d} A=0 .
$$





\section{Appendix B}

\section{Reduction of quasi-static solutions CPU Time in a explicit dynamic code}

VPS-explicit is a explicit dynamic code, therefore the solved equation during the simulations performed in this thesis is

$$
\rho_{0} \frac{\mathrm{D} \boldsymbol{v}}{\mathrm{D} t}-\nabla \cdot \boldsymbol{\sigma}=0
$$

where $\rho_{0}$ indicates the density of the material, $\frac{\mathrm{D} \boldsymbol{v}}{\mathrm{D} t}$ is the material time derivative of the velocity $(\boldsymbol{v}), \boldsymbol{\sigma}$ is the stress tensor. However, due to the low accelerations applied during the experiment, the analyzed problem is quasi-static, i.e. the first term of Eq. B.1 is negligible when compared with the second term, therefore Eq. B.1. is equivalent to:

$$
\nabla \cdot \boldsymbol{\sigma}=0
$$

Consequently, there is no time-dependency in the numerical model. In other words, the time of the simulation has no physical meaning.

In order to model the experimental conditions, the applied boundary conditions were done using an applied displacement ramp from zero at the initial time to the desired final displacement $\left(d_{E}\right)$ at the end of simulation time $\left(t_{E}\right)$. However, this ramp introduces a extremely high acceleration in the first time step calculation producing spurious waves. To avoid this, instead of applied displacement, constant acceleration 


\section{B. Reduction of QuAsi-Static solutions CPU Time in a explicit} DYNAMIC CODE

boundary conditions are applied (time and therefore accelerations have no physical meaning). With these new boundary conditions, the applied acceleration is controlled at any time and no spurious waves are produced (see both results in Fig. B.1). The applied acceleration (a) is obtained from the maximum desired displacement at the end of simulation time, i.e. $a=\frac{2 d_{E}}{t_{E}^{2}}$.

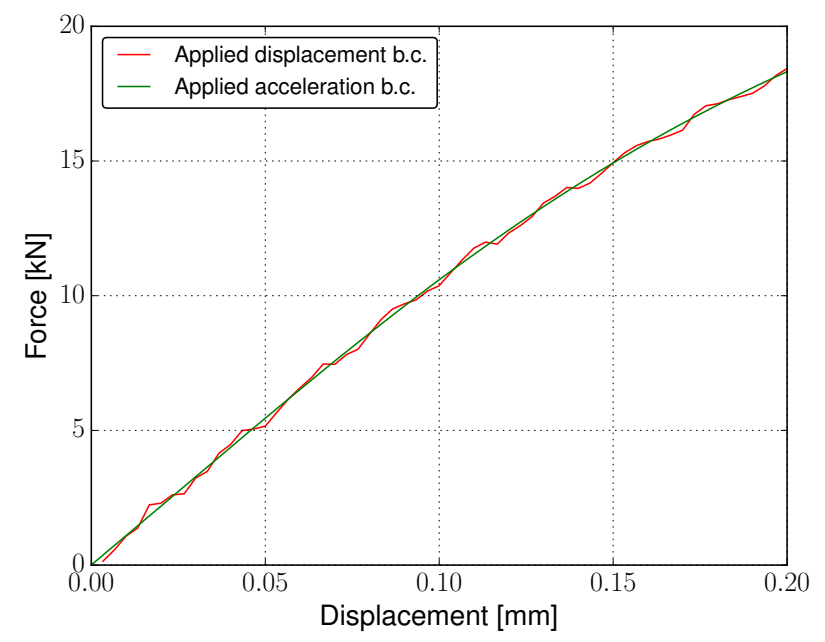

Figure B.1: Obtained force displacement curves depending on type of boundary conditions.

Furthermore, the time step of explicit dynamic codes such as VPS explicit is delimited by the CourantFriedrichsLewy condition [87] defined by:

$$
\Delta t \leq f_{s c} *\left[\frac{h}{c}\right]
$$

where $\Delta t$ is the stable time step, $f_{s c}$ is a scale factor to improve stability, $h$ is the

smallest element dimension in the model, and $c=\sqrt{\frac{E}{\rho}}$ is the acoustic wave speed where $E$ is the Young' modulus.

In order to avoid reduced time steps that would increase the computational costs of large models, VPS code has two mass scaling parameters that let limit the time steps: INITMASSSCALE and DYNAMASSSCALE. When the time step associated with an element through Eq. B.3 is lower to the one given by the mass scaling parameters, the code increses the time step by scaling up the density in the specific elements that are contronlling the time step. Consequently, the code adds a non-physical mass to these elements, that would not disturb the final results if the added mass is not comparable with the initial model mass. INITMASSSCALE is related with the size of 
the elements of the undeformed mesh. DYNAMASSSCALE is related to the elements of the deformed mesh.

Considering all these features, the different not physical terms are modified to reduce computational time $\left(t_{C P U}\right)$. This time depends on the number of time steps $\left(n_{T S}\right)$, that is:

$$
n_{T S}=\frac{t_{E}}{\Delta t}
$$

where $t_{E}$ is the time of the simulation. To reduce $n_{T S}$ and consequently $t_{C P U}$, one option is to increase the $\Delta t$, that is, increasing the limits of INITMASSSCALE, and DYNAMASSSCALE. The threshold for this increase of $\Delta t$ is a too high added numerical mass and therefore a too high non-physical density, that would make the first term of Eq. B.1 of the same magnitude than second term. The second option is to reduce the non-physical $t_{E}$, however due to the relation between this time and the applied accelerations, the first time of Eq. B.1 is also increased leading to a similar threshold in terms of calculation time. 



\section{Bibliography}

[1] Resistance Spot Welding Volkswagen Group Standard - VW 01105-1. (2010-02).

[2] G. P. Nikishkov and S. N. Atluri. Calculation of fracture mechanics parameters for an arbitrary three-dimensional crack, by the "equivalent domain integral" method. International Journal for Numerical Methods in Engineering, 24:18011821, 1987.

[3] S. Keeler and M. Kimchi. Advanced high strength steels for automotive industry. Number 2. WorldAutoSteel, 2014.

[4] M. Pouranvari and S. P. H. Marashi. Critical review of automotive steels spot welding: process, structure and properties. Science and Technology of Welding and Joining, 18(5):361-403, jul 2013.

[5] S. Dancette. Comportement mécanique des soudures par points: mécanismes et stratégies de prédiction dans le cas des tôles en acier pour automobile. $\mathrm{PhD}$ thesis, L'Institut National des Sciences Appliquées de Lyon, 2009.

[6] S. Dancette, D. Fabrègue, V. Massardier, J. Merlin, T. Dupuy, and M. Bouzekri. Experimental and modeling investigation of the failure resistance of Advanced High Strength Steels spot welds. Engineering Fracture Mechanics, 78(10):22592272 , jul 2011.

[7] M. Palmonella and M. I. Friswell. Improving spot weld models in structural dynamics. In Design Engineering Technical Conferences and Computers and Information in Engineering Conference, pages 1-10, Chicago, 2003.

[8] P. K. C. Wood and M. A. Buckley. A new strain rate dependent spot weld model for automotive crash applications. 11th Int. LS-DYNA Users Conference, (2):1$14,2010$.

[9] P. Cruz, J. A. Muñoz, and J. Viñas. Quasi-static Dynamic Spotweld Characterization for Automotive Crashworthiness Conditions. In SIMULIA Community Conference, pages 1-16, 2014. 
[10] S. Dancette, D. Fabrègue, V. Massardier, J. Merlin, T. Dupuy, and M. Bouzekri. Investigation of the Tensile Shear fracture of Advanced High Strength Steel spot welds. Engineering Failure Analysis, 25:112-122, oct 2012.

[11] T. Huin, S. Dancette, D. Fabrègue, and T. Dupuy. Investigation of the Failure of Advanced High Strength Steels Heterogeneous Spot Welds. Metals, 6(5):111, 2016 .

[12] E. H. J. Lamouroux, D. Coutellier, N. Doelle, and P. Kuemmerlen. Detailed model of spot-welded joints to simulate the failure of car assemblies. International Journal on Interactive Design and Manufacturing, 1(1):33-40, mar 2007.

[13] F. Seeger, G. Michel, and M. Blanquet. Investigation of spot weld behavior using detailed modeling technique. In \%. LS-DYNA Anwenderforum, pages 29-38, Bamberg, 2008.

[14] S. Brauser, L. A. Pepke, G. Weber, and M. Rethmeier. Deformation behaviour of spot-welded high strength steels for automotive applications. Materials Science and Engineering: A, 527(26):7099-7108, oct 2010.

[15] S. Sommer. Modeling of the fracture behavior of spot welds using advanced micro-mechanical damage models. In IOP Conference Series: Materials Science and Engineering, volume 10, page 012057, 2010.

[16] S. Burget and S. Sommer. Characterization and modeling of fracture behavior of spot welded joints in hot-stamped ultra-high strength steels. In 11. LS-DYNA Forum, Ulm, 2012.

[17] S. Dancette, D. Fabrègue, R. Estevez, V. Massardier, T. Dupuy, and M. Bouzekri. A finite element model for the prediction of Advanced High Strength Steel spot welds fracture. Engineering Fracture Mechanics, 87:48-61, jun 2012.

[18] T. K. Eller. Modeling of tailor hardened boron steel. PhD thesis, University of Twente, 2016.

[19] F. Krajcarz, Anne-Françoise Gourgues-Lorenzon, Emmanuel Lucas, and André Pineau. Fracture toughness of the molten zone of resistance spot welds. International Journal of Fracture, 181(2):209-226, mar 2013.

[20] P Geck. Advanced High-Strength Steels Add Strength and Ductility to Vehicle Design, 2010.

[21] C. M. Tamarelli. AHSS 101 the evolving use of advance high-strength steel for automotive applications. Technical report, Steel Market Development Institute, Southfield, 2011. 
[22] D. Frómeta, A. Lara, S. Molas, D. Casellas, J. Rehrl, C. Suppan, and P. Larour. Evaluation of crash resistance by means of fracture toughness measurements in Advanced High Strength Steels. In 5th International Conference on Steels in Cars and Trucks, Amsterdam-Schiphol (NL), 2017.

[23] World Steel Association. Automotive Steel Definitions, 2017.

[24] ArcelorMittal. Steels for hot stamping-Usibor @, 2012.

[25] ThysssenKrupp. Dualphasen-Stähle DP-W und DP-K $\{\AA\}\{\AA\}$ 1/16, 2012.

[26] X. Q. Gayden. Development of Requirements for Resistance Spot Welding Dual-Phase (DP600) Steels Part 1 - The Causes of Interfacial Fracture. Welding Journal, 1(November):173-182, 2005.

[27] M. I. Khan, M. L. Kuntz, E. Biro, and Y. Zhou. Microstructure and Mechanical Properties of Resistance Spot Welded Advanced High Strength Steels. Materials Transactions, 49(7):1629-1637, 2008.

[28] Youn-Suk Jong, Young-Kook Lee, Dong-Cheol Kim, Moon-Jin Kang, In-Sung Hwang, and Won-Beom Lee. Microstructural Evolution and Mechanical Properties of Resistance Spot Welded Ultra High Strength Steel Containing Boron. Materials Transactions, 52(6):1330-1333, 2011.

[29] S. Dancette, V. Massardier-Jourdan, D. Fabrègue, J. Merlin, T. Dupuy, and M. Bouzekri. HAZ Microstructures and Local Mechanical Properties of High Strength Steels Resistance Spot Welds. ISIJ International, 51(1):99-107, 2011.

[30] G. Shi and S. A. Westgate. Resistance spot welding of high strength steels. International Journal for the Joining of Materials, 16(1):9-14, 2004.

[31] H.-S. Choi, G.-H. Park, W.-S. Lim, and B.-min Kim. Evaluation of weldability for resistance spot welded single-lap joint between GA780DP and hotstamped 22MnB5 steel sheets. Journal of Mechanical Science and Technology, 25(6):1543-1550, aug 2011.

[32] Y. J. Chao. Ultimate Strength and Failure Mechanism of Resistance Spot Weld Subjected to Tensile, Shear, or Combined Tensile/Shear Loads. Journal of Engineering Materials and Technology, 125(2):125, 2003.

[33] V. J. Badheka, S. K. Agrawal, and N. Shroll. Mode of failure of resistance spot welded martenstic stainless steel-Part II. International Journal of Mechanical and Materials Engineering, 5(1):43-52, 2010.

[34] M. Pouranvari, S. P. H. Marashi, and D. S. Safanama. Failure mode transition in AHSS resistance spot welds. Part II: Experimental investigation and model validation. Materials Science and Engineering: A, 528(29-30):8344-8352, nov 2011. 
[35] M. Pouranvari and S. P. H. Marashi. Failure mode transition in AHSS resistance spot welds. Part I. Controlling factors. Materials Science and Engineering: A, 528(29-30):8337-8343, nov 2011.

[36] O. Hahn, D. Gieske, and A. Rhode. Probe und Probenspannvorrichtung zum Einsatz in Zugmaschinen, 1996.

[37] Specification for AutomotiveWeld Quality-Resistance SpotWelding of Steel, 2007.

[38] M. Pouranvari, S. P. H. Marashi, and S. M. Mousavizadeh. Failure mode transition and mechanical properties of similar and dissimilar resistance spot welds of DP600 and low carbon steels. Science and Technology of Welding and Joining, 15(7):625-631, 2010.

[39] T. K. Eller, L. Greve, M. Andres, M. Medricky, T. Meinders, and T. van den Boogaard. Identification of Plasticity Model Parameters of the Heat-Affected Zone in Resistance Spot Welded Martensitic Boron Steel. Key Engineering Materials, 639:369-376, 2015.

[40] B. Langrand and A. Combescure. Non-linear and failure behaviour of spotwelds: a global finite element and experiments in pure and mixed modes I/II. International Journal of Solids and Structures, 41(24-25):6631-6646, dec 2004.

[41] A. Combescure, F. Delcroix, L. Caplain, S. Espanol, and P. Eliot. A finite element to simulate the failure of weld points on impact. International Journal of Impact Engineering, 28(7):783-802, 2003.

[42] N. Khandoker and M. Takla. Tensile strength and failure simulation of simplified spot weld models. Materials \& Design, 54:323-330, feb 2014.

[43] S.-H. Lin, J. Pan, T. Tyan, and P. Prasad. A general failure criterion for spot welds under combined loading conditions. International Journal of Solids and Structures, 40(21):5539-5564, oct 2003.

[44] T. L. Anderson. Fracture Mechanics: Fundamentals and Applications, Third Edition. CRC Taylor \& Francis, 2005.

[45] Yuh J. Chao. Failure mode of spot welds: interfacial versus pullout. Science and Technology of Welding and Joining, 2003.

[46] F. Seeger, M. Feucht, G. Dumitru, and T. Graf. Enhancement of Spot Weld Modeling using MAT 100 DAI. In 7. LS-DYNA Anwenderforum, pages 1-12, Bamberg, 2008. 
[47] S. Marzi, O. Hesebeck, M. Brede, and F. Kleiner. A Rate-Dependent, ElastoPlastic Cohesive Zone Mixed-Mode Model for Crash Analysis of Adhesively Bonded Joints Stress Unloading Path. In 7th European LS-DSYNA Conference, 2009.

[48] T. Heubrandtner, G. Rangger, and D. Scherjau. Advanced Spotweld Failure Modelling Based on Trefftz Formulation. In 4. LS-DYNA Anwenderforum, pages 13-22, Bamberg, 2005.

[49] C. Thibaud. Recent Developments in PAM-CRASH/SAFE V2006, 2006.

[50] X. Deng, W. Chen, and G. Shi. Three-dimensional finite element analysis of the mechanical behavior of spot welds. Finite Elements in Analysis and Design, $35: 17-39,2000$.

[51] M. Tumuluru and D. J. Radakovic. Predicting resistance spot weld failure modes in shear tension tests of advanced high-strength automotive steels. Welding Journal, 87(April):96-105, 2008.

[52] M. Tumuluru and D. J. Radakovic. An Evaluation of the Cross-Tension Test of Resistance Spot Welds in High-Strength Dual-Phase Steels, 2012.

[53] Y. P. Yang, S. S. Babu, F. Orth, and W. Peterson. Integrated computational model to predict mechanical behaviour of spot weld. Science and Technology of Welding and Joining, 13(3):232-239, 2008.

[54] D. Pieronek, R. Peter, and A. Marx. Ganzheitlicher Simulationsansatz zur Versagensprognose von widerstandspunktgeschweißten Stahlblechverbindungen Inhalt Einleitung, 2009.

[55] David Pieronek, Andre Marx, and R P Röttger. Holistischer Ansatz zur Versagensmodellierung von Schwei $\{ß\}$ punkten in der Crashsimulation. In 9. LSDYNA Anwenderforum, pages 13-24, Bamberg, 2010.

[56] D. P. Norman. Modelling Spotweld Fracture Using CrachFEM. In 9th European LS-DYNA Conference, Manchester, 2013.

[57] Forschungsvereinigung Automobiltechnik, Forschungsvereinigung Stahlanwendung, Franz-josef Heise Das Forschungsvorhaben, and Werkstoffmechanik Iwm. Charakterisierung und Ersatzmodellierung des Bruchverhaltens von Crashsimulation unter Berücksichtigung der Auswirkung der Verbindung auf das Bauteilverhalten. Technical report, FOSTA-Forschungsvereinigung Stahlanwendung, 2012.

[58] S. Burget and S. Sommer. Modeling of deformation and failure behavior of dissimilar resistance spot welded joints under shear, axial and combined loading conditions. ICF13, pages 1-12, 2013. 
[59] W. Tong, H. Tao, X. Jiang, N. Zhang, M. P. Marya, L. G. Hector, and X. Q. Gayden. Deformation and Fracture of Miniature Tensile Bars with Resistance- Spot- Weld Microstructures. Metallurgical and Materials Transactions A, 36(October):2651-2669, 2005.

[60] R. Lacroix. Caracterisation de la rupture interfaciale de points soudes d'aciers a tres haute. PhD thesis, Ecole Nationale Supérieure des Mines - Saint Etienne, 2011.

[61] E. Feulvarch, V. Robin, and J. M. Bergheau. Resistance spot welding simulation: a general finite element formulation of electrothermal contact conditions. Journal of Materials Processing Technology, 153-154:436-441, nov 2004.

[62] Wen Zheng and Min Wang. Parameter optimization of dissimilar resistance spot welding on ultra-high strength hot-stamped steel and mild steel by numerical simulation. Acta Metall. Mater., 25(6):487-498, 2012.

[63] K. Tahar Chaouch and Ph. Rogeon. Development of weld nugget in dissymmetric assemblies. In 21ème Congrès Français de Mécanique, pages 1-6, 2013.

[64] T. K. Eller, L. Greve, M. Andres, M. Medricky, H. J. M. Geijselaers, V. T. Meinders, and A. H. Van Den Boogaard. Plasticity and fracture modeling of the heat-affected zone in resistance spot welded tailor hardened boron steel. Journal of Materials Processing Technology, 234:309-322, 2016.

[65] B. Zhou, M. D. Thouless, and S. M. Ward. Predicting the failure of ultrasonic spot welds by pull-out from sheet metal. International Journal of Solids and Structures, 43(25-26):7482-7500, dec 2006.

[66] M. N. Cavalli, M. D. Thouless, and Q. D. Yang. Cohesive-zone modelling of the deformation and fracture of spot-welded joints. Fatigue and Fracture of Engineering Materials and Structures, 28(10):861-874, oct 2005.

[67] Yuanli Bai and Tomasz Wierzbicki. A new model of metal plasticity and fracture with pressure and Lode dependence. International Journal of Plasticity, 2008.

[68] Yuanli Bai and Tomasz Wierzbicki. Application of extended Mohr-Coulomb criterion to ductile fracture. International Journal of Fracture, 161(1):1-20, nov 2009.

[69] Yaning Li, Meng Luo, Jörg Gerlach, and Tomasz Wierzbicki. Prediction of shear-induced fracture in sheet metal forming. Journal of Materials Processing Technology, 210(14):1858-1869, nov 2010.

[70] H. Dell, H. Gese, and G. Oberhofer. CrachFEM-A Comprehensive Approach For The Prediction Of Sheet Metal Failure. AIP Conference Proceedings, 2007. 
[71] H. Gese, Gernot Oberhofer, and Harry Dell. Consistent Modelling of Plasticity and Failure in the Process Chain of Deep Drawing and Crash with User Material Model MF-GenYld+ CrachFEM for LS-. In LS-DYNA Anwenderforum, pages 13-24, Frankenthal, 2007. DYNAmore GmbH.

[72] A. L. Gurson. Continuum theory of ductile rupture by void nucleation and growth: Part 1-Yield criteria and flow rules for porous ductile media. Journal of Engineering Materials and Technology, Transactions of the ASME, 99 Ser $\mathrm{H}(1): 2-15,1977$.

[73] M. Gologanu, J.-b. Leblond, and J. Devaux. Approximate models for ductile metals containing non-spherical voids. Case of axisymmetric prolate ellipsoidal cavities. Journal of the Mechanics and Physics of Solids, 41(11):1723-1754, nov 1993.

[74] Bin Zhou, M D Thouless, and S M Ward. Determining Mode-I Cohesive Parameters for Nugget Fracture in Ultrasonic Spot Welds. Int. J. Fract., 136(14):309-326, nov 2005.

[75] A. A. Griffith. The Phenomena of Rupture and Flow in Solids. Philosophical Transactions of the Royal Society A: Mathematical, Physical and Engineering Sciences, 221(582-593):163-198, 1921.

[76] G. Irwin. Analysis of stresses and strains near the end of a crack traversing a plate. Journal of Applied Mechanics, 24:361-364, 1957.

[77] A. Pandolfi and M. Ortiz. An eigenerosion approach to brittle fracture. International Journal for Numerical Methods in Engineering, 92(June):694-714, 2012.

[78] J. R. Rice. A Path Independent Integral and the Approximate Analysis of Strain Concentration by Notches and Cracks. Journal of Applied Mechanics, 35(2):379, 1968.

[79] H. G. DeLorenzi. On the energy release rate and the J-integral for 3-D crack configurations. International Journal of Fracture, 19(3):183-193, 1982.

[80] F. Krajcarz, E. Lucas, M. Mazière, and A. Pineau. Local Approach Applied to the Fracture Toughness of Resistance Spot Welds. In 13th International Conference on Fracture, number 2004, pages 1-10, Beijing, 2013.

[81] Ibrahim Sevim. Effect of hardness to fracture toughness for spot welded steel sheets. Materials $\&$ Design, 27(1):21-30, jan 2004.

[82] Rémi Lacroix, Joël Monatte, Arnaud Lens, Guillaume Kermouche, J. M. Bergheau, and Helmut Klöcker. Spot Weld Strength Determination Using the 
Wedge Test: In Situ Observations and Coupled Simulations. Applied Mechanics and Materials, 24-25:299-304, jun 2010.

[83] Y. W. Shi, N. N. Zhou, and J. X. Zhang. Comparison of mode I and mode II elastic-plastic fracture toughness for two low alloyed high strength steels. International Journal of Fracture, 68:89-97, 1994.

[84] N. M. Abd-Allah, M.S. El-Fadaly, M. M. Megahed, and A. M. Eleiche. Fracture Toughness Properties of High-Strength Martensitic Steel within a Wide Hardness Range. Journal of Materials Engineering and Performance, 10:576-585, 2001 .

[85] PAM-SCL - Theory Notes Manual, 2000.

[86] L. Greve. Modulare Materialmodellierung fur die Simulation von Deformationsund Bruchvorgänges. In crashMAT 2012 - 6. Freiburg Workshop zum Werkstoffund Strukturverhalten bei Crashvorgängen., 2012.

[87] H. Lewy, K. Friedrichs, and R. Courant. Über die partiellen Differenzengleichungen der mathematischen Physik. Mathematische Annalen, 100:32-74, 1928.

[88] T. Belytschko, W.K. Liu, and B. Moran. Nonlinear Finite Elements for Continua ans Structures. Wiley, 2000.

[89] T. K. Eller, L. Greve, M. T. Andres, and M. Medricky. Constitutive modeling of quench-hardenable boron steel with tailored properties. In Form. Technol. Forum, pages 1-6, TUM, 2013.

[90] T. K. Eller, L. Greve, M. Andres, M. Medricky, A. Hatscher, V. T. Meinders, and A. H. Van Den Boogaard. Plasticity and fracture modeling of quenchhardenable boron steel with tailored properties. Journal of Materials Processing Technology, 214(6):1211-1227, 2014.

[91] Bernd Schmidt, Fernando Fraternali, and Michael Ortiz. Eigenfracture: an eigendeformation approach to variational fracture. Multiscale Modeling 85 Simulation, 7(3):1237-1266, 2009.

[92] a. Pandolfi, B Li, and M Ortiz. Modeling fracture by material-point erosion. Int. J. Fract., 184(1-2):3-16, nov 2012.

[93] F. Z. Li, C. F. Shih, and A. Needleman. A comparison of methods for calculating energy release rates. Engineering Failure Analysis, 21(2):405-421, 1985.

[94] C. F. Shih, B. Moran, and T. Nakamura. Energy Release Rate Along a ThreeDimensional Crack Front in a Thermally Stressed Body. International Journal of Fracture, 30(2):79-102, 1986. 
[95] G. P. Nikishkov and S. N. Atluri. An equivalent domain integral method for computing crack-tip integral parameters in non-elastic, thermo-mechanical fracture. Engineering Fracture Mechanics, 26(6):851-867, 1987.

[96] N. K. Simha, F. D. Fischer, G. X. Shan, C. R. Chen, and O. Kolednik. J-integral and crack driving force in elastic-plastic materials. Journal of the Mechanics and Physics of Solids, 2008.

[97] O. Kolednik, R. Schöngrundner, and F. D. Fischer. A new view on J-integrals in elastic-plastic materials. International Journal of Fracture, 187(1):77-107, 2014.

[98] L. Xue and T. Belytschko. Fast methods for determining instabilities of elasticplastic damage models through closed-form expressions. International Journal for Numerical Methods in Engineering, 84(June):1490-1518, 2010.

[99] O. Sherepenko and S. Jüttner. Schweißtechnische Verarbeitung höchstfester Stähle: Widerstandspunktschweißen unter Berücksichtigung fertigungs- bedingter Spalte, 2013.

[100] S. Sommer. Modellierung des Verformungs-und Versagensverhaltens von Punktschweißverbindungen unter monoton ansteigender Belastung. PhD thesis, Universität Karlsruhe, 2009.

[101] P. Gumbsch, S. Sommer, S Burget, E. Roos, H.-J. Wink, D. Krätschmer, O. Hahn, G. Meschut, F. Klokkers, and D. Hein. Charakterisierung und Ersatzmodellierung des Bruchverhaltens von Punktschweißverbindungen an ultrahochfesten Stählen für die Crashsimulation unter Berücksichtigung der Auswirkung der Verbindung auf das Bauteilverhalten. Final Report. Technical report, FOSTA/AVIF P 806/ A262, 2013.

[102] T. K. Eller, L. Greve, M. T. Andres, M. Medricky, and V. T. Meinders. The softened heat-affected zone in resistance spot welded tailor hardened boron steel: a material model for crash simulation. In International Conference of Impact Loading of Structures and Materials, pages 3-6, 2016.

[103] H. W. Swift. Plastic instability under plane stress. Journal of Mechanics Physics and Solids, 1, 1952.

[104] E. Voce. A practical strain-hardening function. Metallurgica, 51:219-226, 1955.

[105] Borja Erice, Christian Roth, and Dirk Mohr. Effect of the loading rate and stress state on the ductile fracture initiation of the Dual Phase 980 Advanced High Strength Steel. In Anales de la Mecánica de la Fractura, San Sebastián, 2016. 